\title{
Selectivity in Gas Adsorption by Molecular Cucurbit[6]uril
}

Sudip Pan, ${ }^{\dagger}$ Ranajit Saha, ${ }^{\dagger}$ Subhajit Mandal, ${ }^{\dagger}$ Sukanta Mondal, ${ }^{\S}$ Ashutosh Gupta, ${ }^{\dagger, \ddagger}$

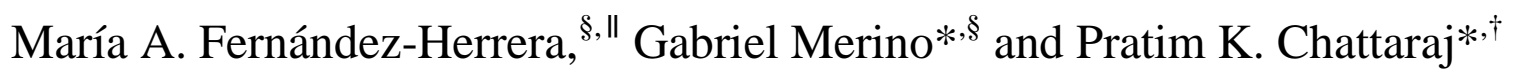

${ }^{\dagger}$ Department of Chemistry and Centre for Theoretical Studies, Indian Institute of Technology Kharagpur, Kharagpur 721302, India

${ }^{\ddagger}$ Department of Chemistry, Udai Pratap Autonomous College, Varanasi, Uttar Pradesh 221002 India

${ }^{\S}$ Departamento de Física Aplicada, Centro de Investigación y de Estudios Avanzados Unidad Mérida. km 6 Antigua carretera a Progreso. Apdo. Postal 73, Cordemex, 97310, Mérida, Yuc., México

"Conacyt Research Fellow: Departamento de Física Aplicada, Centro de Investigación y de Estudios Avanzados Unidad Mérida, km 6 Antigua carretera a Progreso. Apdo. Postal 73, Cordemex, 97310 Mérida, Yuc., Mexico

* Corresponding authors: gmerino@cinvestav.mx (G.M.), pkc@chem.iitkgp.ernet.in (P.K.C.) 
Table 1-SI. Comparison of bond distance (in $\AA$ ) between atoms of gas molecules in their isolated state and encapsulated state within $\mathrm{CB}[6]$

\begin{tabular}{|l|l|l|}
\hline Molecule & Isolated & CB[6] \\
\hline $\mathrm{C}_{2} \mathrm{H}_{2}$ & $\begin{array}{l}\text { C-C (triple): } 1.203 \\
\text { C-H: } 1.066\end{array}$ & $\begin{array}{l}\text { C-C (triple): } 1.206 \\
\text { C-H: } 1.070\end{array}$ \\
\hline $\mathrm{C}_{2} \mathrm{H}_{6}$ & $\begin{array}{l}\text { C-C (single): } 1.526 \\
\text { C-H: } 1.094\end{array}$ & $\begin{array}{l}\text { C-C (single): } 1.528 \\
\text { C-H: } 1.095\end{array}$ \\
\hline $\mathrm{C}_{2} \mathrm{H}_{4}$ & $\begin{array}{l}\text { C-C (double): } 1.327 \\
\text { C-H: } 1.086\end{array}$ & $\begin{array}{l}\text { C-C (double): } 1.330 \\
\text { C-H: } 1.088\end{array}$ \\
\hline $\mathrm{CO}_{2}$ & C-O: 1.165 & C-O: 1.165 \\
\hline $\mathrm{Cl}_{2}$ & Cl-Cl: 2.010 & Cl-Cl: 2.014 \\
\hline $\mathrm{CH}_{4}$ & C-H: 1.091 & C-H: 1.091 \\
\hline $\mathrm{F}_{2}$ & F-F: 1.386 & F-F: 1.389 \\
\hline $\mathrm{CO}$ & C-O: 1.135 & C-O: 1.136 \\
\hline $\mathrm{NO}_{2}$ & N-O: 1.195 & N-O: 1.195 \\
\hline $\mathrm{H}_{2} \mathrm{~S}$ & H-S: 1.342 & H-S: 1.345 \\
\hline $\mathrm{N}_{2}$ & N-N: 1.102 & N-N: 1.102 \\
\hline $\mathrm{NO}$ & N-O: 1.153 & N-O: 1.153 \\
\hline $\mathrm{H}_{2}$ & H-H: 0.743 & H-H: 0.745 \\
\hline $\mathrm{H}_{2} \mathrm{O}$ & O-H: 0.960 & O-H: 0.965 \\
\hline $\mathrm{CS} \mathrm{S}_{2}$ & C-S: 1.556 & C-S: 1.552 \\
\hline $\mathrm{Br} 2$ & Br-Br: 2.288 & Br-Br: 2.292 \\
\hline $\mathrm{HF}$ & H-F: 0.919 & H-F: 0.932 \\
\hline $\mathrm{HCl}$ & H-Cl: 1.281 & H-Cl: 1.290 \\
\hline $\mathrm{HBr}$ & H-Br: 1.410 & H-Br: 1.417 \\
\hline
\end{tabular}


Table 2-SI. $\Delta \mathrm{G}_{298 \mathrm{~K}}$ values (in $\mathrm{kcal} / \mathrm{mol}$ ) for the processes computed at the $\omega \mathrm{B} 97 \mathrm{X}-\mathrm{D} / 6-31 \mathrm{G}(\mathrm{d}, \mathrm{p})$ level.

\begin{tabular}{|c|c|}
\hline Process & $\Delta \mathrm{G}_{298 \mathrm{~K}}$ \\
\hline $\mathrm{C}_{2} \mathrm{H}_{2}+\mathrm{Cl}_{2} @ \mathrm{CB}[6] \rightarrow \mathrm{Cl}_{2}+\mathrm{C}_{2} \mathrm{H}_{2} @ \mathrm{CB}[6]$ & 1.1 \\
\hline $\mathrm{C}_{2} \mathrm{H}_{2}+\mathrm{C}_{2} \mathrm{H}_{6} @ \mathrm{CB}[6] \rightarrow \mathrm{C}_{2} \mathrm{H}_{6}+\mathrm{C}_{2} \mathrm{H}_{2} @ \mathrm{CB}[6]$ & -0.7 \\
\hline $\mathrm{C}_{2} \mathrm{H}_{2}+\mathrm{Br}_{2} @ \mathrm{CB}[6] \rightarrow \mathrm{Br}_{2}+\mathrm{C}_{2} \mathrm{H}_{2} @ \mathrm{CB}[6]$ & -0.8 \\
\hline $\mathrm{C}_{2} \mathrm{H}_{2}+\mathrm{CS}_{2} @ \mathrm{CB}[6] \rightarrow \mathrm{CS}_{2}+\mathrm{C}_{2} \mathrm{H}_{2} @ \mathrm{CB}[6]$ & -2.6 \\
\hline $\mathrm{C}_{2} \mathrm{H}_{2}+\mathrm{H}_{2} \mathrm{O} @ \mathrm{CB}[6] \rightarrow \mathrm{H}_{2} \mathrm{O}+\mathrm{C}_{2} \mathrm{H}_{2} @ \mathrm{CB}[6]$ & -2.7 \\
\hline $\mathrm{C}_{2} \mathrm{H}_{2}+\mathrm{HCl} @ \mathrm{CB}[6] \rightarrow \mathrm{HCl}+\mathrm{C}_{2} \mathrm{H}_{2} @ \mathrm{CB}[6]$ & -3.1 \\
\hline $\mathrm{C}_{2} \mathrm{H}_{2}+\mathrm{CO}_{2} @ \mathrm{CB}[6] \rightarrow \mathrm{CO}_{2}+\mathrm{C}_{2} \mathrm{H}_{2} @ \mathrm{CB}[6]$ & -3.2 \\
\hline $\mathrm{C}_{2} \mathrm{H}_{2}+\mathrm{C}_{2} \mathrm{H}_{4} @ \mathrm{CB}[6] \rightarrow \mathrm{C}_{2} \mathrm{H}_{4}+\mathrm{C}_{2} \mathrm{H}_{2} @ \mathrm{CB}[6]$ & -3.5 \\
\hline $\mathrm{C}_{2} \mathrm{H}_{2}+\mathrm{HBr} @ \mathrm{CB}[6] \rightarrow \mathrm{HBr}+\mathrm{C}_{2} \mathrm{H}_{2} @ \mathrm{CB}[6]$ & -3.6 \\
\hline $\mathrm{C}_{2} \mathrm{H}_{2}+\mathrm{CH} \mathrm{H}_{4} @ \mathrm{CB}[6] \rightarrow \mathrm{CH}_{4}+\mathrm{C}_{2} \mathrm{H}_{2} @ \mathrm{CB}[6]$ & -3.8 \\
\hline $\mathrm{C}_{2} \mathrm{H}_{2}+\mathrm{H}_{2} \mathrm{~S} @ \mathrm{CB}[6] \rightarrow \mathrm{H}_{2} \mathrm{~S}+\mathrm{C}_{2} \mathrm{H}_{2} @ \mathrm{CB}[6]$ & -4.1 \\
\hline $\mathrm{C}_{2} \mathrm{H}_{2}+\mathrm{F}_{2} @ \mathrm{CB}[6] \rightarrow \mathrm{F}_{2}+\mathrm{C}_{2} \mathrm{H}_{2} @ \mathrm{CB}[6]$ & -4.8 \\
\hline $\mathrm{C}_{2} \mathrm{H}_{2}+\mathrm{CO} @ \mathrm{CB}[6] \rightarrow \mathrm{CO}+\mathrm{C}_{2} \mathrm{H}_{2} @ \mathrm{CB}[6]$ & -5.1 \\
\hline $\mathrm{C}_{2} \mathrm{H}_{2}+\mathrm{HF} @ \mathrm{CB}[6] \rightarrow \mathrm{HF}+\mathrm{C}_{2} \mathrm{H}_{2} @ \mathrm{CB}[6]$ & -6.0 \\
\hline $\mathrm{C}_{2} \mathrm{H}_{2}+\mathrm{NO} \mathrm{O}_{2} @ \mathrm{CB}[6] \rightarrow \mathrm{NO}_{2}+\mathrm{C}_{2} \mathrm{H}_{2} @ \mathrm{CB}[6]$ & -6.1 \\
\hline $\mathrm{C}_{2} \mathrm{H}_{2}+\mathrm{N}_{2} @ \mathrm{CB}[6] \rightarrow \mathrm{N}_{2}+\mathrm{C}_{2} \mathrm{H}_{2} @ \mathrm{CB}[6]$ & -6.4 \\
\hline $\mathrm{C}_{2} \mathrm{H}_{2}+\mathrm{NO} @ \mathrm{CB}[6] \rightarrow \mathrm{NO}_{2} \mathrm{C}_{2} \mathrm{H}_{2} @ \mathrm{CB}[6]$ & -7.5 \\
\hline $\mathrm{C}_{2} \mathrm{H}_{2}+\mathrm{H}_{2} @ \mathrm{CB}[6] \rightarrow \mathrm{H}_{2}+\mathrm{C}_{2} \mathrm{H}_{2} @ \mathrm{CB}[6]$ & -10.2 \\
\hline
\end{tabular}


Table 3-SI. Energy decomposition analysis of gas@CB[6]. All the energies are in kcal/mol.

\begin{tabular}{|c|c|c|c|c|c|}
\hline Gas@CB6 & $\Delta \mathrm{E}_{\text {int }}$ & $\Delta \mathrm{E}_{\text {elstat }}$ & $\Delta \mathrm{E}_{\text {Pauli }}$ & $\Delta \mathrm{E}_{\text {orb }}$ & $\Delta \mathrm{E}_{\text {disp }}$ \\
\hline $\mathrm{C}_{2} \mathrm{H}_{2}$ & -14.7 & $-10.4(48.4)$ & 6.6 & $-2.2(10.3)$ & $-8.8(41.3)$ \\
\hline $\mathrm{C}_{2} \mathrm{H}_{6}$ & -14.2 & $-5.4(24.9)$ & 7.6 & $-2.8(12.9)$ & $-13.5(62.1)$ \\
\hline $\mathrm{C}_{2} \mathrm{H}_{4}$ & -13.6 & $-7.5(33.1)$ & 8.9 & $-2.8(12.5)$ & $-12.3(54.3)$ \\
\hline $\mathrm{CO}_{2}$ & -10.1 & $-4.4(30.1)$ & 4.5 & $-2.0(13.8)$ & $-8.2(56.1)$ \\
\hline $\mathrm{Cl}_{2}$ & -13.1 & $-5.2(32.1)$ & 2.9 & $-2.1(12.9)$ & $-8.8(54.9)$ \\
\hline $\mathrm{CH}_{4}$ & -8.8 & $-3.5(24.7)$ & 5.2 & $-2.0(14.4)$ & $-8.5(60.9)$ \\
\hline $\mathrm{F}_{2}$ & -6.7 & $-2.4(26.8)$ & 2.3 & $-1.5(16.0)$ & $-5.2(57.3)$ \\
\hline $\mathrm{CO}$ & -7.1 & $-2.7(24.9)$ & 3.6 & $-2.1(19.2)$ & $-6.0(55.9)$ \\
\hline $\mathrm{NO}_{2}$ & -10.2 & $-3.8(24.2)$ & 5.4 & $-3.7(23.9)$ & $-8.1(51.9)$ \\
\hline $\mathrm{H}_{2} \mathrm{~S}$ & -12.2 & $-7.6(43.4)$ & 5.2 & $-3.5(19.9)$ & $-6.4(36.7)$ \\
\hline $\mathrm{N}_{2}$ & -7.5 & $-2.3(21.2)$ & 3.1 & $-2.2(20.9)$ & $-6.2(57.9)$ \\
\hline $\mathrm{NO}_{\mathrm{H}}$ & -5.1 & $-2.1(20.7)$ & 5.1 & $-2.6(25.1)$ & $-5.5(54.1)$ \\
\hline $\mathrm{H}_{2}$ & -3.2 & $-1.8(31.1)$ & 2.4 & $-0.9(16.2)$ & $-3.0(52.7)$ \\
\hline $\mathrm{C} \mathrm{O}$ & -12.5 & $-12.6(53.5)$ & 12.6 & $-6.0(25.2)$ & $-5.0(21.3)$ \\
\hline $\mathrm{Cr}$ & -12.3 & $-5.2(19.4)$ & 14.4 & $-6.4(24.0)$ & $-15.1(56.6)$ \\
\hline $\mathrm{BF}$ & -18.9 & $-11.4(34.3)$ & 14.3 & $-5.6(16.8)$ & $-16.3(48.9)$ \\
\hline $\mathrm{HCl}$ & -12.0 & $-13.4(57.4)$ & 11.4 & $-4.9(20.7)$ & $-5.1(21.9)$ \\
\hline $\mathrm{HBr}$ & -11.6 & $-8.2(48.7)$ & 5.2 & $-2.3(13.9)$ & $-6.3(37.3)$ \\
\hline $\mathrm{HCl}$ & -12.9 & $-10.4(44.5)$ & 10.5 & $-3.0(12.7)$ & $-10.0(42.9)$ \\
\hline
\end{tabular}

The values within parentheses show the percentage contribution towards the total attraction, $\Delta \mathrm{E}_{\text {elstat }}+\Delta \mathrm{E}_{\text {orb }}+\Delta \mathrm{E}_{\text {disp. }}$. 


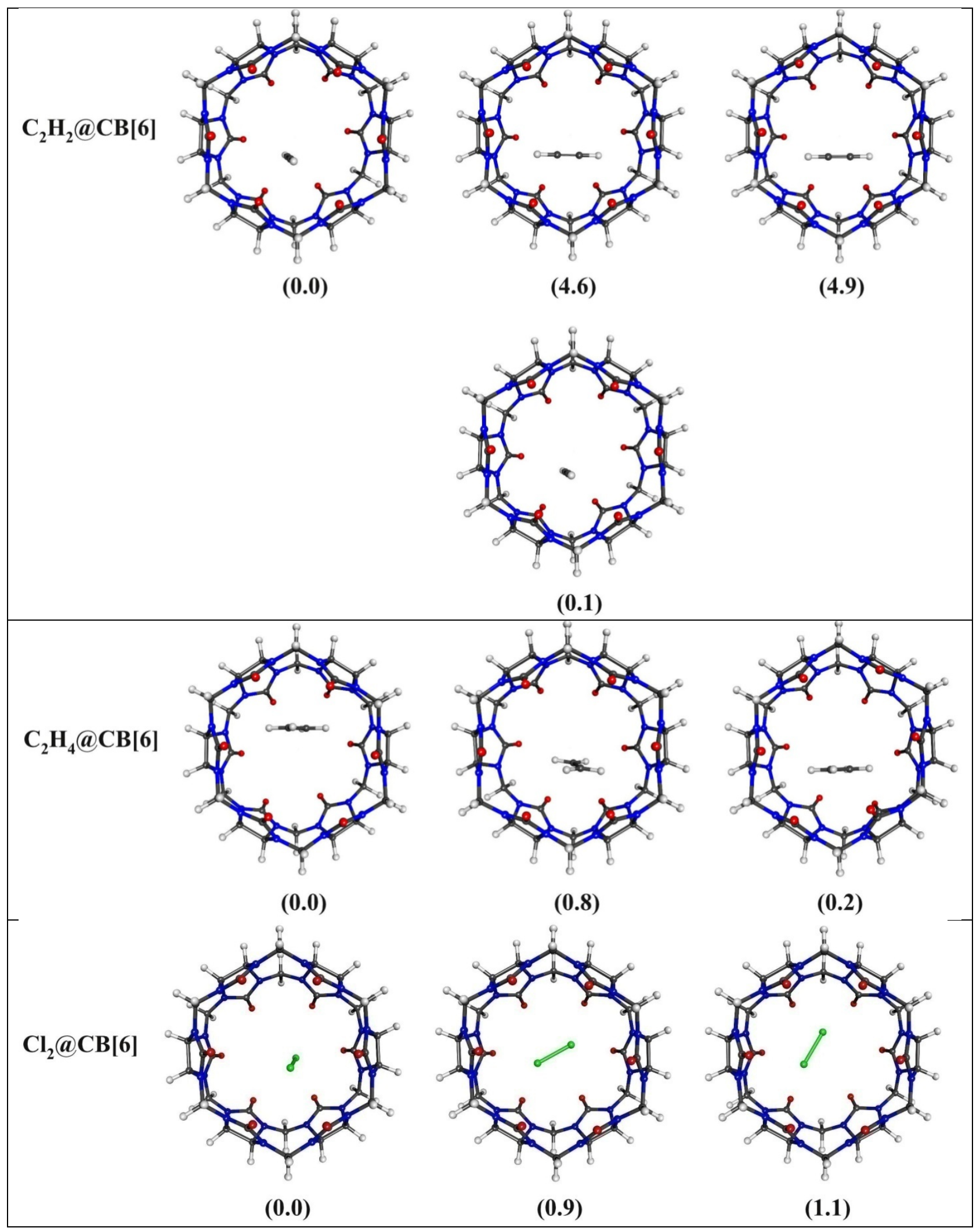




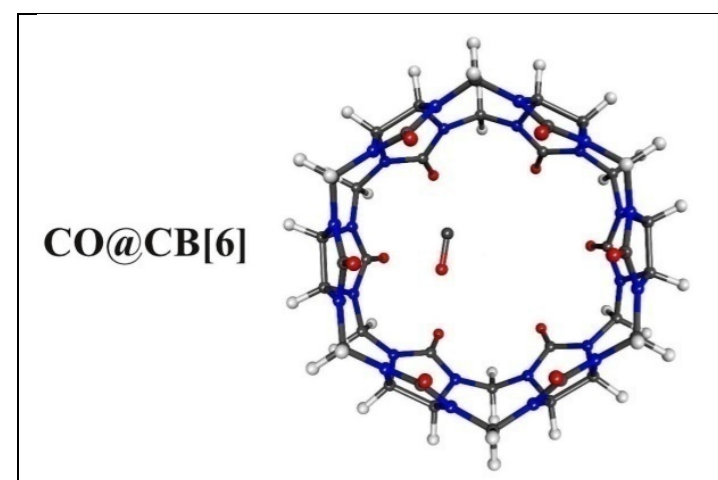

(0.0)

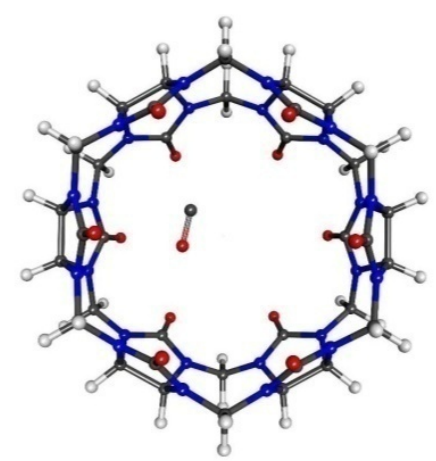

(0.5)

(0.5)

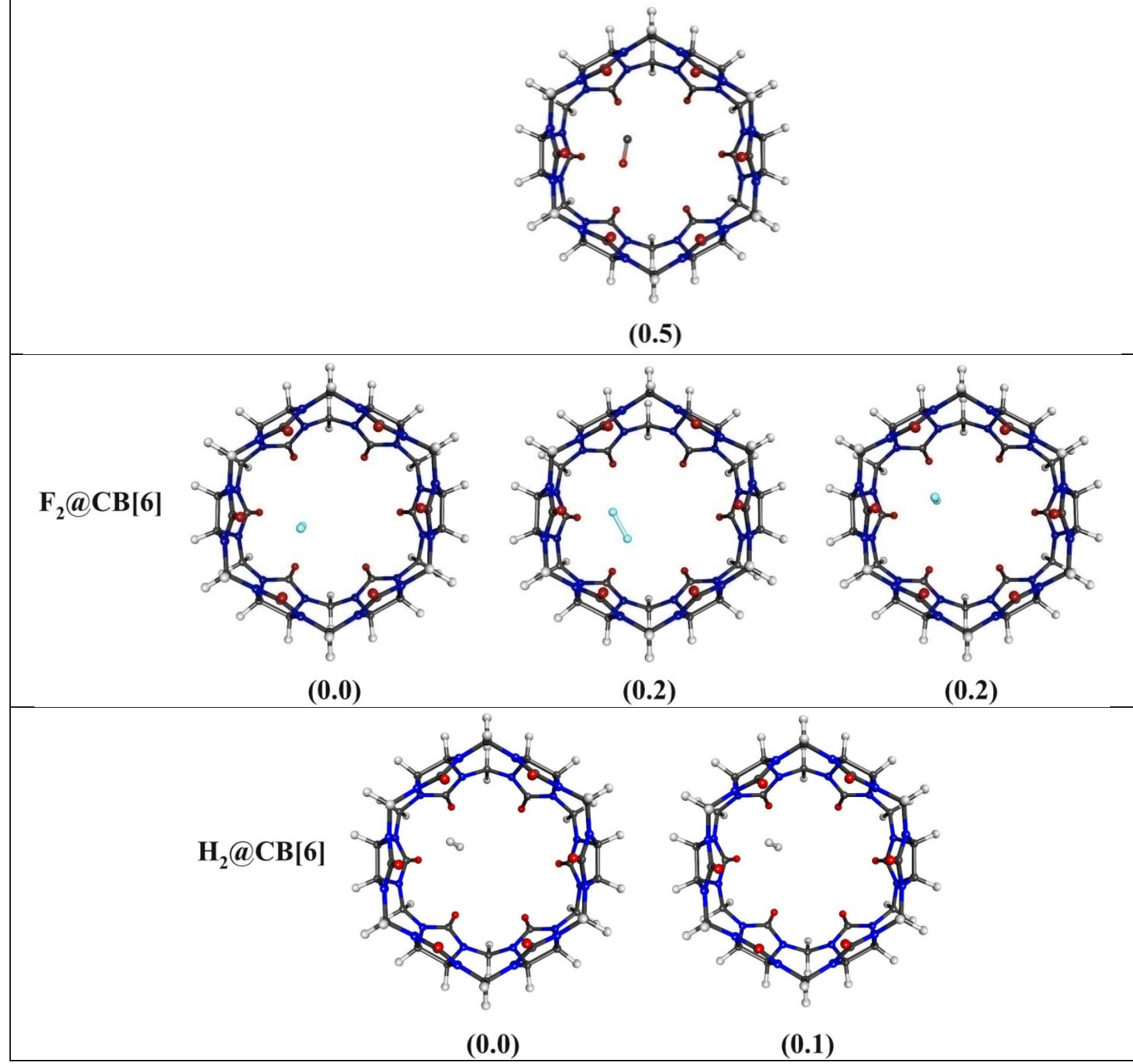

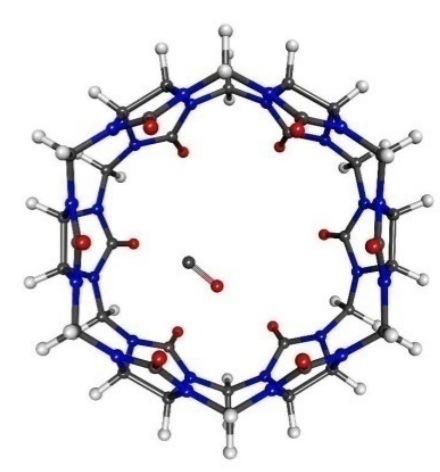

(0.5) 


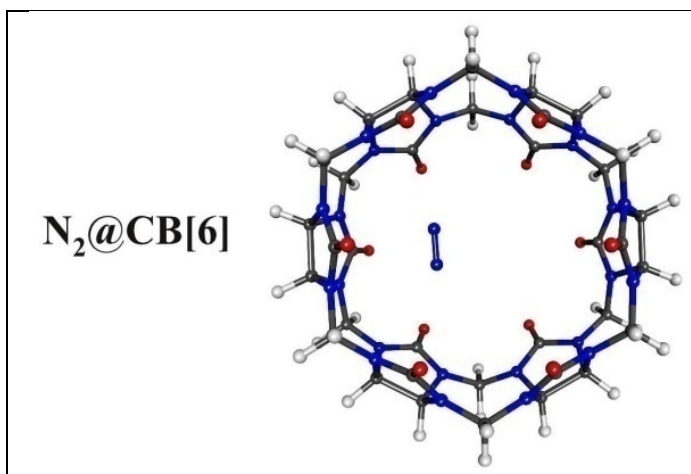

(0.0)

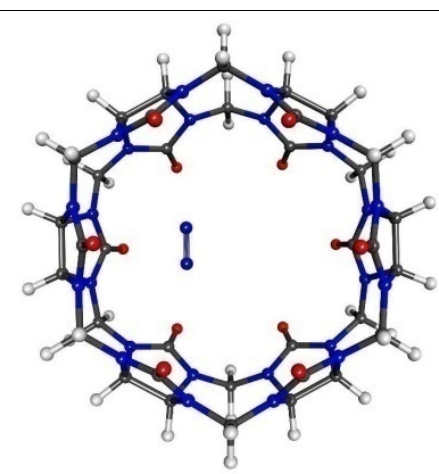

(0.1)

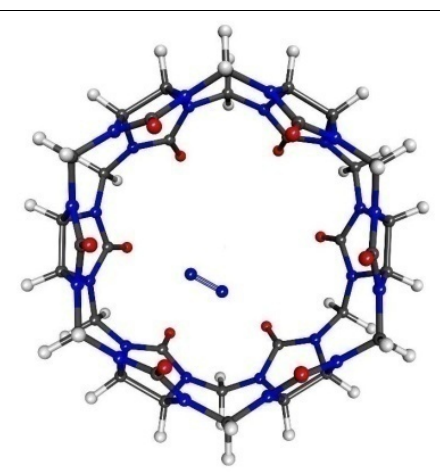

$(0.1)$

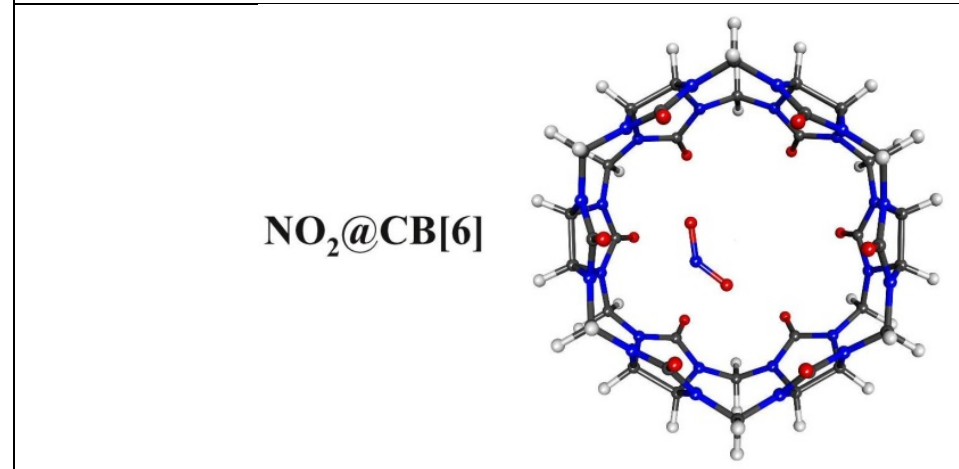

(0.0)

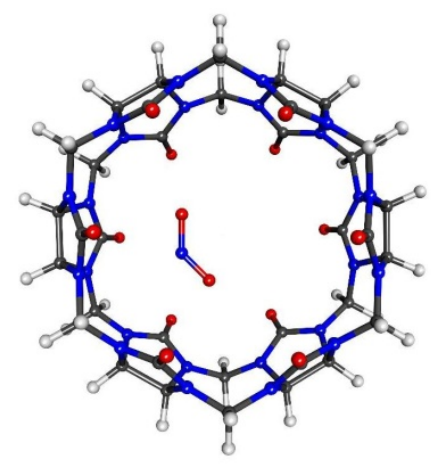

(0.1)

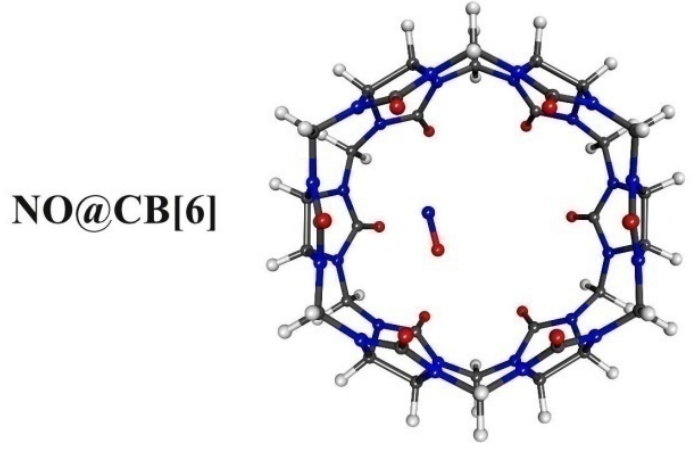

(0.0)

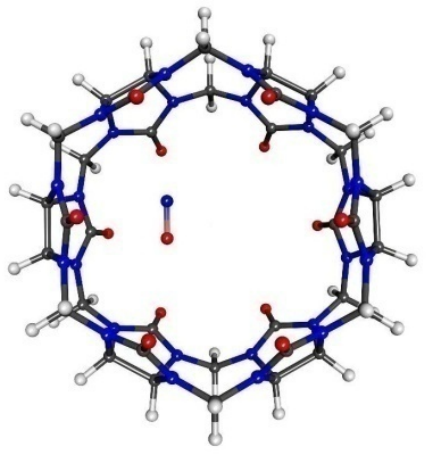

(0.4)

Figure 1-SI.The different isomers of gas@CB[6] obtained at the $\omega$ B97X-D/6-31G(d,p) level. 

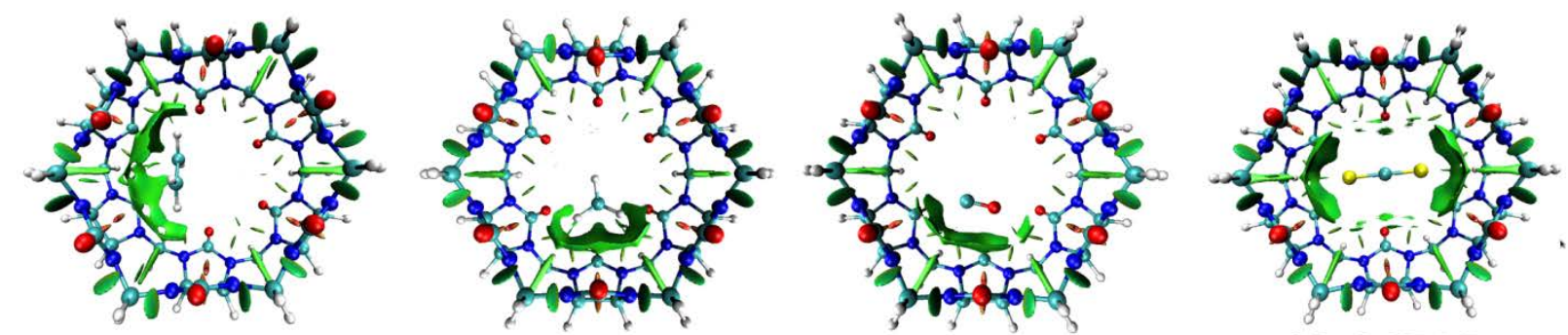

$$
\mathrm{C}_{2} \mathrm{H}_{4} @ \mathrm{CB}[6
$$

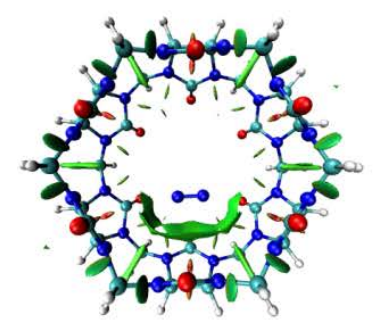

$\mathrm{N}_{2} @ \mathrm{CB}[6]$

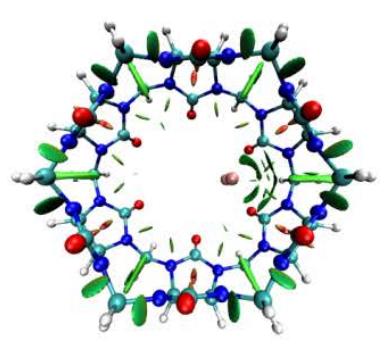

$\mathrm{F}_{2} @ \mathrm{CB}[6]$
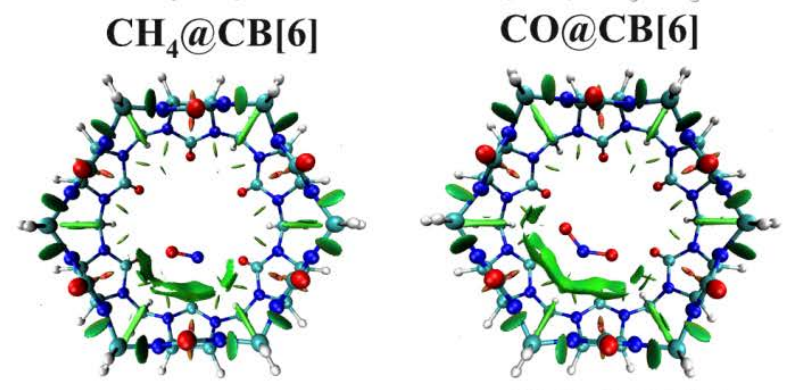

$\mathrm{NO}_{2} @ \mathrm{CB}[6]$

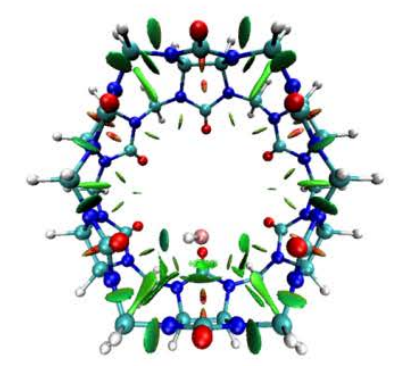

HF@CB[6]

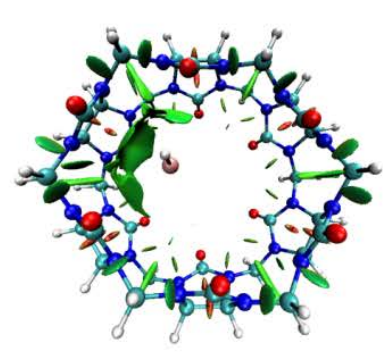

$\mathrm{HBr} @ \mathrm{CB}[6]$

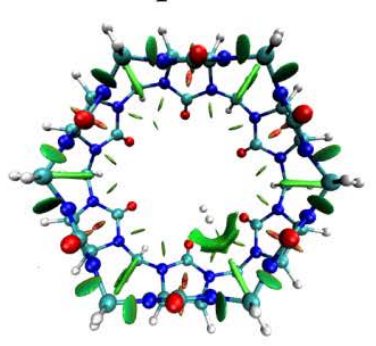

$\mathrm{H}_{2} @ \mathrm{CB}[6]$

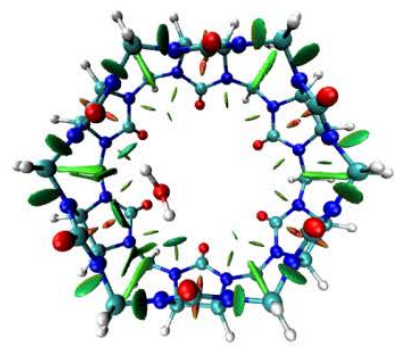

$\mathrm{H}_{2} \mathrm{O} @ \mathrm{CB}[6]$

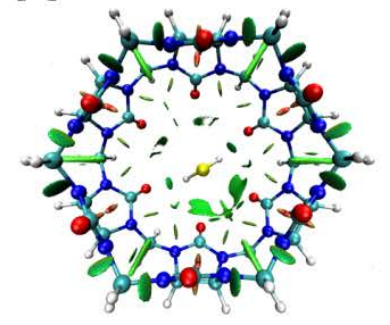

$\mathrm{H}_{2} \mathrm{~S} @ \mathrm{CB}[6]$

Figure 2-SI. NCI isosurface plots of gas@CB[6]. 

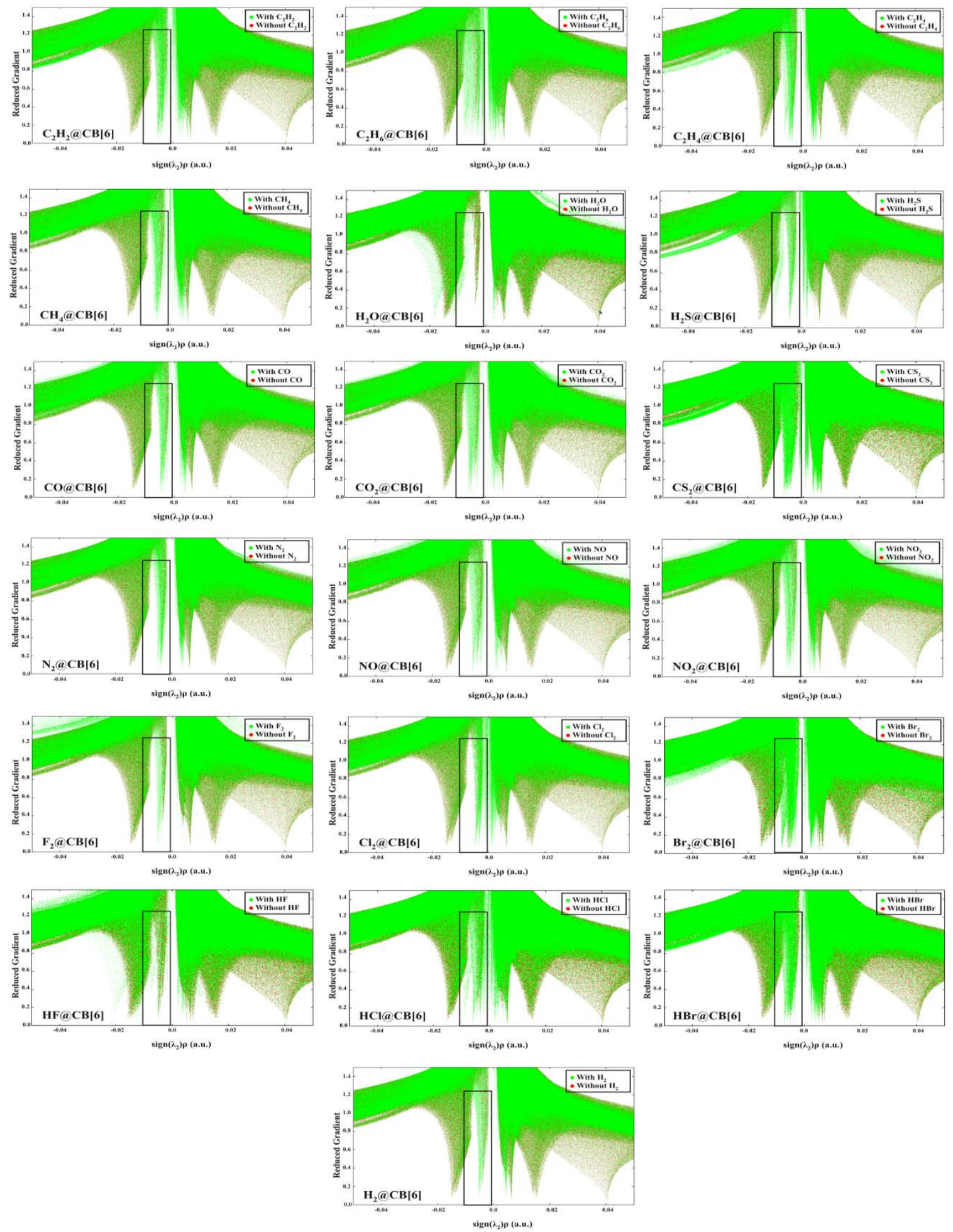

Figure 3-SI. Zoomed view of the plots of reduced gradient versus sign $\left(\lambda_{2}\right) \rho$ of the gas@CB[6] systems. The rectangular boxes indicates the appearance of attractive interactions in presence of the gases with the $\mathrm{CB}[6]$ moiety. 


\begin{tabular}{|c|c|c|c|}
\hline \multicolumn{4}{|c|}{ Cartesian coordinates of gas@CB[6] } \\
\hline \multicolumn{4}{|c|}{$\mathrm{CB}[6]$} \\
\hline $\mathrm{C}$ & 5.05881100 & 0.62302100 & 0.07553000 \\
\hline C & 4.62460000 & 2.12500500 & 0.10248500 \\
\hline $\mathrm{N}$ & 3.80244900 & 2.21016000 & 1.28080700 \\
\hline $\mathrm{C}$ & 3.77904700 & 1.03293500 & 2.01101900 \\
\hline $\mathrm{N}$ & 4.50892800 & 0.09898600 & 1.29401600 \\
\hline $\mathrm{N}$ & 4.49393600 & 0.13641300 & -1.15796500 \\
\hline $\mathrm{H}$ & 6.15063100 & 0.48594400 & 0.06690600 \\
\hline $\mathrm{N}$ & 3.96764300 & 2.30315300 & -1.16349800 \\
\hline $\mathrm{H}$ & 5.47263900 & 2.82134600 & 0.18458500 \\
\hline $\mathrm{C}$ & 3.28030000 & 3.43405200 & 1.82214100 \\
\hline $\mathrm{O}$ & 3.27360900 & 0.86953200 & 3.09552800 \\
\hline $\mathrm{C}$ & 3.51759100 & 3.56384900 & -1.69042800 \\
\hline $\mathrm{C}$ & 3.94350400 & 1.14889900 & -1.93064900 \\
\hline $\mathrm{O}$ & 3.57038300 & 1.05611400 & -3.07511300 \\
\hline $\mathrm{N}$ & 2.23271600 & 4.01002800 & -1.22310300 \\
\hline C & 1.98523900 & 4.68073600 & 0.02169700 \\
\hline C & 1.08403300 & 3.82304000 & -1.97592700 \\
\hline C & 0.47119200 & 5.05593800 & -0.06632900 \\
\hline $\mathrm{N}$ & 2.05266300 & 3.88190200 & 1.22059000 \\
\hline $\mathrm{H}$ & 2.64497700 & 5.55642400 & 0.11372900 \\
\hline $\mathrm{N}$ & 0.03188100 & 4.35755900 & -1.24871900 \\
\hline $\mathrm{O}$ & 1.02312100 & 3.34285800 & -3.08231800 \\
\hline $\mathrm{N}$ & -0.05647300 & 4.60344700 & 1.18999600 \\
\hline $\mathrm{H}$ & 0.29711500 & 6.13637100 & -0.17957600 \\
\hline C & 0.87924400 & 3.92772700 & 1.95819100 \\
\hline $\mathrm{O}$ & 0.71921700 & 3.51274200 & 3.08075600 \\
\hline C & -1.26730800 & 4.51653400 & -1.84287800 \\
\hline $\mathrm{C}$ & -1.39349500 & 4.83842100 & 1.66712900 \\
\hline $\mathrm{N}$ & -2.40248600 & 3.94144000 & 1.17001700 \\
\hline $\mathrm{N}$ & -2.29335700 & 3.69069100 & -1.26658700 \\
\hline C & -2.88112100 & 2.89088100 & 1.93693300 \\
\hline $\mathrm{C}$ & -3.06040000 & 4.05336600 & -0.10150800 \\
\hline $\mathrm{C}$ & -4.15703400 & 2.94255900 & -0.03969600 \\
\hline $\mathrm{N}$ & -3.83359100 & 2.23038800 & 1.17282000 \\
\hline C & -2.88345500 & 2.67354000 & -2.00075400 \\
\hline $\mathrm{N}$ & -3.98257900 & 2.23757000 & -1.27752000 \\
\hline C & -4.69119900 & 1.24153600 & 1.77469900 \\
\hline $\mathrm{C}$ & -4.79906000 & 1.14788000 & -1.73574300 \\
\hline $\mathrm{O}$ & -2.54384600 & 2.28341800 & -3.09184700 \\
\hline $\mathrm{O}$ & -2.57136300 & 2.63659500 & 3.07583100 \\
\hline $\mathrm{N}$ & -4.50574100 & -0.09850900 & 1.29358800 \\
\hline $\mathrm{N}$ & -4.49596400 & -0.13624900 & -1.15831600 \\
\hline C & -3.77932600 & -1.03389900 & 2.01208600 \\
\hline $\mathrm{C}$ & -5.05838300 & -0.62258200 & 0.07627700 \\
\hline C & -4.62462200 & -2.12476000 & 0.10254000 \\
\hline $\mathrm{N}$ & -3.80581100 & -2.21168300 & 1.28290700 \\
\hline $\mathrm{O}$ & -3.27403200 & -0.87114100 & 3.09677600 \\
\hline
\end{tabular}




\begin{tabular}{|c|c|c|c|}
\hline C & -3.28241100 & -3.43554400 & 1.82301700 \\
\hline $\mathrm{N}$ & -3.96425000 & -2.30164000 & -1.16196800 \\
\hline $\mathrm{C}$ & -3.94352600 & -1.14806800 & -1.93033800 \\
\hline $\mathrm{O}$ & -3.57128600 & -1.05536600 & -3.07508600 \\
\hline C & -3.51594900 & -3.56272400 & -1.68966000 \\
\hline $\mathrm{N}$ & -2.05401200 & -3.88182100 & 1.22162200 \\
\hline $\mathrm{N}$ & -2.23184300 & -4.01095700 & -1.22222600 \\
\hline C & -0.88074800 & -3.92613300 & 1.95965100 \\
\hline C & -1.98532400 & -4.68113500 & 0.02311300 \\
\hline C & -0.47114500 & -5.05621700 & -0.06358000 \\
\hline $\mathrm{N}$ & 0.05572200 & -4.60182700 & 1.19241600 \\
\hline $\mathrm{O}$ & -0.72143500 & -3.50999800 & 3.08189700 \\
\hline C & 1.39283100 & -4.83515500 & 1.67011900 \\
\hline $\mathrm{N}$ & -0.03116800 & -4.35953200 & -1.24668000 \\
\hline C & -1.08272300 & -3.82479800 & -1.97456200 \\
\hline $\mathrm{O}$ & -1.02113400 & -3.34521500 & -3.08117700 \\
\hline C & 1.26812500 & -4.52001300 & -1.84031300 \\
\hline $\mathrm{N}$ & 2.40101400 & -3.93809900 & 1.17163300 \\
\hline $\mathrm{N}$ & 2.29443500 & -3.69354200 & -1.26562000 \\
\hline C & 3.06033800 & -4.05313600 & -0.09899000 \\
\hline $\mathrm{C}$ & 2.88296400 & -2.67574500 & -2.00005000 \\
\hline C & 4.15700900 & -2.94214000 & -0.03876900 \\
\hline $\mathrm{N}$ & 3.98064200 & -2.23711800 & -1.27632400 \\
\hline C & 2.88189300 & -2.88852300 & 1.93848500 \\
\hline $\mathrm{N}$ & 3.83554700 & -2.22994300 & 1.17416200 \\
\hline $\mathrm{O}$ & 2.54324600 & -2.28699300 & -3.09159300 \\
\hline $\mathrm{O}$ & 2.57265700 & -2.63348200 & 3.07734500 \\
\hline C & 4.79681100 & -1.14774100 & -1.73560300 \\
\hline C & 4.69420200 & -1.24122100 & 1.77485000 \\
\hline $\mathrm{H}$ & -5.85208500 & 1.39275700 & -1.53945100 \\
\hline $\mathrm{H}$ & -4.63128000 & 1.05558500 & -2.81194900 \\
\hline $\mathrm{H}$ & -4.46127800 & 1.23164800 & 2.84343400 \\
\hline $\mathrm{H}$ & -5.74091400 & 1.52931200 & 1.61889700 \\
\hline $\mathrm{H}$ & -4.04274000 & -4.22449900 & 1.72251700 \\
\hline $\mathrm{H}$ & -3.07034100 & -3.25479300 & 2.88037800 \\
\hline $\mathrm{H}$ & -4.26364500 & -4.33273300 & -1.45694400 \\
\hline $\mathrm{H}$ & -3.43066400 & -3.43730500 & -2.77207800 \\
\hline $\mathrm{H}$ & 1.68124300 & -5.86482000 & 1.42124800 \\
\hline $\mathrm{H}$ & 1.36686900 & -4.70459200 & 2.75496900 \\
\hline $\mathrm{H}$ & 1.57239600 & -5.57518900 & -1.76542900 \\
\hline $\mathrm{H}$ & 1.17428400 & -4.23226100 & -2.89107800 \\
\hline $\mathrm{H}$ & 4.46555200 & -1.23099800 & 2.84384300 \\
\hline $\mathrm{H}$ & 5.74360900 & -1.52928800 & 1.61782700 \\
\hline $\mathrm{H}$ & 4.62825000 & -1.05564600 & -2.81172100 \\
\hline $\mathrm{H}$ & 5.85002800 & -1.39255600 & -1.53999200 \\
\hline $\mathrm{H}$ & 4.04154500 & 4.22231600 & 1.72268200 \\
\hline $\mathrm{H}$ & 3.06750100 & 3.25260900 & 2.87924000 \\
\hline $\mathrm{H}$ & 3.43287900 & 3.43880200 & -2.77291900 \\
\hline $\mathrm{H}$ & 4.26402400 & 4.33487600 & -1.45714700 \\
\hline $\mathrm{H}$ & -3.47344200 & 5.06598600 & -0.22221200 \\
\hline $\mathrm{H}$ & -5.17939400 & 3.34434500 & 0.02279700 \\
\hline $\mathrm{H}$ & -5.47300700 & -2.82106900 & 0.18146100 \\
\hline $\mathrm{H}$ & -6.15016600 & -0.48516800 & 0.06979300 \\
\hline $\mathrm{H}$ & -2.64499500 & -5.55687700 & 0.11499400 \\
\hline $\mathrm{H}$ & -0.29691200 & -6.13679100 & -0.17528600 \\
\hline $\mathrm{H}$ & 5.17952500 & -3.34380600 & 0.02213100 \\
\hline $\mathrm{H}$ & -1.57192800 & 5.57178800 & -1.77029200 \\
\hline $\mathrm{H}$ & -1.17322700 & 4.22653700 & -2.89300600 \\
\hline $\mathrm{H}$ & -1.36765100 & 4.70935500 & 2.75216700 \\
\hline $\mathrm{H}$ & -1.68113800 & 5.86791100 & 1.41676200 \\
\hline $\mathrm{H}$ & 3.47352500 & -5.06606500 & -0.21674700 \\
\hline
\end{tabular}




\begin{tabular}{|c|c|c|c|}
\hline \multicolumn{4}{|c|}{ C $\mathrm{C}_{2} @ \mathrm{CB}[6]$} \\
\hline $\mathrm{C}$ & 1.42397300 & -4.78558800 & -0.13112000 \\
\hline $\mathrm{C}$ & 2.86232600 & -4.18402200 & -0.06170900 \\
\hline $\mathrm{N}$ & 2.84151600 & -3.42743000 & 1.16759600 \\
\hline $\mathrm{C}$ & 1.71878400 & -3.70163100 & 1.93658900 \\
\hline $\mathrm{N}$ & 0.87661700 & -4.48153500 & 1.16037900 \\
\hline $\mathrm{N}$ & 0.83875800 & -4.10794600 & -1.26067000 \\
\hline $\mathrm{H}$ & 1.41441300 & -5.87359300 & -0.29612300 \\
\hline $\mathrm{N}$ & 2.96568400 & -3.44169700 & -1.28425000 \\
\hline $\mathrm{H}$ & 3.65201700 & -4.94870800 & -0.01908800 \\
\hline $\mathrm{C}$ & 4.01361200 & -2.84669300 & 1.77034100 \\
\hline $\mathrm{O}$ & 1.53832500 & -3.37803800 & 3.08594900 \\
\hline $\mathrm{C}$ & 4.13625900 & -2.74028800 & -1.73148600 \\
\hline $\mathrm{C}$ & 1.77031800 & -3.37869600 & -1.98365000 \\
\hline $\mathrm{O}$ & 1.59523400 & -2.84690600 & -3.05358100 \\
\hline $\mathrm{N}$ & 4.33686300 & -1.44244600 & -1.14028000 \\
\hline $\mathrm{C}$ & 5.04177200 & -1.21669100 & 0.09860500 \\
\hline C & 4.25548500 & -0.29766400 & -1.91990300 \\
\hline $\mathrm{C}$ & 5.17852500 & 0.33916100 & 0.15087500 \\
\hline $\mathrm{N}$ & 4.34408900 & -1.52606600 & 1.31380000 \\
\hline $\mathrm{H}$ & 6.01061500 & -1.73734300 & 0.07953900 \\
\hline $\mathrm{N}$ & 4.71779900 & 0.75434800 & -1.14478800 \\
\hline $\mathrm{O}$ & 3.90933200 & -0.23837100 & -3.07495900 \\
\hline $\mathrm{N}$ & 4.36384700 & 0.70105500 & 1.28309900 \\
\hline $\mathrm{H}$ & 6.21292600 & 0.67938900 & 0.30708300 \\
\hline $\mathrm{C}$ & 3.93272000 & -0.39918600 & 2.00680400 \\
\hline $\mathrm{O}$ & 3.36377500 & -0.38119900 & 3.07194000 \\
\hline $\mathrm{C}$ & 4.72173600 & 2.10441100 & -1.64050400 \\
\hline $\mathrm{C}$ & 4.29822700 & 2.02253400 & 1.84447900 \\
\hline $\mathrm{N}$ & 3.32580600 & 2.88368200 & 1.22990600 \\
\hline $\mathrm{N}$ & 3.63054700 & 2.93548200 & -1.20517500 \\
\hline $\mathrm{C}$ & 2.23463300 & 3.34815200 & 1.94834000 \\
\hline $\mathrm{C}$ & 3.57119300 & 3.65431500 & 0.03495800 \\
\hline $\mathrm{C}$ & 2.26653700 & 4.49869000 & -0.10006800 \\
\hline $\mathrm{N}$ & 1.61429500 & 4.30809700 & 1.16339600 \\
\hline $\mathrm{C}$ & 2.48058400 & 3.08147900 & -1.96596800 \\
\hline $\mathrm{N}$ & 1.63018300 & 3.91615800 & -1.25733200 \\
\hline $\mathrm{C}$ & 0.44969100 & 5.01963300 & 1.61829600 \\
\hline $\mathrm{C}$ & 0.45936800 & 4.46131200 & -1.88698600 \\
\hline $\mathrm{O}$ & 2.28549400 & 2.61997300 & -3.06471200 \\
\hline $\mathrm{O}$ & 1.92090800 & 3.02685000 & 3.06925500 \\
\hline $\mathrm{N}$ & -0.81502200 & 4.53155800 & 1.13925600 \\
\hline $\mathrm{N}$ & -0.78343100 & 4.08661200 & -1.27029600 \\
\hline $\mathrm{C}$ & -1.64335200 & 3.75522500 & 1.93543600 \\
\hline $\mathrm{C}$ & -1.36921300 & 4.79368100 & -0.15871400 \\
\hline $\mathrm{C}$ & -2.80236700 & 4.18251600 & -0.06793100 \\
\hline $\mathrm{N}$ & -2.77220700 & 3.46920200 & 1.18393200 \\
\hline $\mathrm{O}$ & -1.44773000 & 3.44719400 & 3.08665800 \\
\hline $\mathrm{C}$ & -3.91916700 & 2.84030600 & 1.77971900 \\
\hline $\mathrm{N}$ & -2.90007700 & 3.39162500 & -1.25982700 \\
\hline $\mathrm{C}$ & -1.70512600 & 3.30872000 & -1.95319000 \\
\hline $\mathrm{O}$ & -1.52080100 & 2.72384600 & -2.99518500 \\
\hline $\mathrm{C}$ & -4.09533200 & 2.74492600 & -1.72817100 \\
\hline $\mathrm{N}$ & -4.21992100 & 1.52386100 & 1.28946900 \\
\hline $\mathrm{N}$ & -4.36995600 & 1.45603200 & -1.15702400 \\
\hline $\mathrm{C}$ & -3.83202900 & 0.39251500 & 1.98233400 \\
\hline
\end{tabular}




\begin{tabular}{|c|c|c|c|}
\hline $\mathrm{C}$ & -4.99824400 & 1.22844900 & 0.11813200 \\
\hline $\mathrm{C}$ & -5.14785500 & -0.32962600 & 0.16717100 \\
\hline $\mathrm{N}$ & -4.36375900 & -0.69596200 & 1.31639900 \\
\hline $\mathrm{O}$ & -3.20170200 & 0.36345900 & 3.01444900 \\
\hline $\mathrm{C}$ & -4.27242600 & -2.01882500 & 1.86636300 \\
\hline $\mathrm{N}$ & -4.66186000 & -0.74953000 & -1.11954000 \\
\hline $\mathrm{C}$ & -4.21743800 & 0.30090200 & -1.90140500 \\
\hline $\mathrm{O}$ & -3.83341100 & 0.23195400 & -3.04614100 \\
\hline $\mathrm{C}$ & -4.65679600 & -2.09870100 & -1.61595500 \\
\hline $\mathrm{N}$ & -3.28729000 & -2.86808300 & 1.25519300 \\
\hline $\mathrm{N}$ & -3.56858000 & -2.93053400 & -1.17869300 \\
\hline $\mathrm{C}$ & -3.51746100 & -3.64543600 & 0.06273900 \\
\hline $\mathrm{C}$ & -2.40964000 & -3.06663700 & -1.92543800 \\
\hline $\mathrm{C}$ & -2.20824900 & -4.48563200 & -0.05896800 \\
\hline $\mathrm{N}$ & -1.57576400 & -3.92464700 & -1.22708500 \\
\hline $\mathrm{C}$ & -2.17028200 & -3.28434700 & 1.95964200 \\
\hline $\mathrm{N}$ & -1.55301100 & -4.26353100 & 1.19863000 \\
\hline $\mathrm{O}$ & -2.19844500 & -2.58660800 & -3.01410200 \\
\hline $\mathrm{O}$ & -1.83221900 & -2.91045800 & 3.05826900 \\
\hline $\mathrm{C}$ & -0.41329500 & -4.48653300 & -1.85681800 \\
\hline $\mathrm{C}$ & -0.38288500 & -4.95857000 & 1.66311900 \\
\hline $\mathrm{H}$ & 0.53310600 & 5.56027700 & -1.90501100 \\
\hline $\mathrm{H}$ & 0.44287600 & 4.07270300 & -2.90903000 \\
\hline $\mathrm{H}$ & 0.42649400 & 4.92458900 & 2.70675000 \\
\hline $\mathrm{H}$ & 0.55368100 & 6.07527100 & 1.33528200 \\
\hline $\mathrm{H}$ & -4.80364100 & 3.47626300 & 1.63084000 \\
\hline $\mathrm{H}$ & -3.70238400 & 2.74636400 & 2.84694500 \\
\hline $\mathrm{H}$ & -4.94500900 & 3.41510200 & -1.53953600 \\
\hline $\mathrm{H}$ & -3.97503100 & 2.58636500 & -2.80269900 \\
\hline $\mathrm{H}$ & -5.25880900 & -2.49988800 & 1.79324900 \\
\hline $\mathrm{H}$ & -3.98686000 & -1.90987200 & 2.91598500 \\
\hline $\mathrm{H}$ & -5.59943300 & -2.58596600 & -1.33573100 \\
\hline $\mathrm{H}$ & -4.58223600 & -2.02560800 & -2.70364700 \\
\hline $\mathrm{H}$ & -0.35011600 & -4.83465000 & 2.74834800 \\
\hline $\mathrm{H}$ & -0.48737600 & -6.02173200 & 1.40955600 \\
\hline $\mathrm{H}$ & -0.40125100 & -4.11712400 & -2.88601200 \\
\hline $\mathrm{H}$ & -0.49640800 & -5.58505300 & -1.85353300 \\
\hline $\mathrm{H}$ & 4.87710200 & -3.50349700 & 1.59437500 \\
\hline $\mathrm{H}$ & 3.81234400 & -2.77246700 & 2.84213200 \\
\hline $\mathrm{H}$ & 4.02101300 & -2.57979500 & -2.80647000 \\
\hline $\mathrm{H}$ & 5.01555900 & -3.36993700 & -1.53672400 \\
\hline $\mathrm{H}$ & 4.47771900 & 4.26548400 & 0.15675800 \\
\hline $\mathrm{H}$ & 2.45428600 & 5.56968600 & -0.26827800 \\
\hline $\mathrm{H}$ & -3.59994800 & 4.94003600 & -0.05385200 \\
\hline $\mathrm{H}$ & -1.36796400 & 5.87605900 & -0.35622000 \\
\hline $\mathrm{H}$ & -5.96014900 & 1.75961500 & 0.16430600 \\
\hline $\mathrm{H}$ & -6.18725600 & -0.66304200 & 0.30098200 \\
\hline $\mathrm{H}$ & -2.38953400 & -5.56097400 & -0.20364100 \\
\hline $\mathrm{H}$ & 5.66389200 & 2.58778900 & -1.35326800 \\
\hline $\mathrm{H}$ & 4.65292700 & 2.03410500 & -2.72881900 \\
\hline $\mathrm{H}$ & 4.00707900 & 1.91309900 & 2.89281300 \\
\hline $\mathrm{H}$ & 5.29236800 & 2.49012700 & 1.77761200 \\
\hline $\mathrm{H}$ & -4.42294700 & -4.25891900 & 0.17981700 \\
\hline $\mathrm{C}$ & -1.18527200 & -0.08655600 & -0.89293600 \\
\hline $\mathrm{H}$ & -1.24026700 & 0.04874200 & -1.95344500 \\
\hline $\mathrm{C}$ & -1.13748300 & -0.24312500 & 0.30195400 \\
\hline $\mathrm{H}$ & -1.09076500 & -0.36949200 & 1.36351200 \\
\hline
\end{tabular}




\section{$\mathrm{C}_{2} \mathrm{H}_{4} @ \mathrm{CB}[6]$}

\begin{tabular}{|c|c|c|c|}
\hline & -0.73535100 & 4.91763200 & 0.08666700 \\
\hline & 0.81765500 & 4.92449900 & -0.04419000 \\
\hline & 1.04108300 & 4.12292300 & -1.22319200 \\
\hline & -0.12471600 & 3.90192800 & -1.94168700 \\
\hline & -1.17238900 & 4.37646300 & -1.16684100 \\
\hline & -0.95409400 & 4.10165700 & 1.25712600 \\
\hline & -1.16693200 & 5.91895900 & 0.23534600 \\
\hline & 1.25763100 & 4.37816900 & 1.20708300 \\
\hline & 1.24144500 & 5.93043200 & -0.18353500 \\
\hline & 2.32638400 & 3.97224300 & -1.84935100 \\
\hline & -0.20909900 & 3.44014900 & -3.05418200 \\
\hline & 2.62294900 & 4.31899600 & 1.65735100 \\
\hline & 0.21352500 & 3.89200700 & 1.97862500 \\
\hline & 0.29863400 & 3.43070600 & 3.09104300 \\
\hline & 3.40218100 & 3.21994800 & 1.15511700 \\
\hline & 4.02302300 & 3.16184200 & -0.13836800 \\
\hline & 3.65421500 & 2.09770100 & 1.92863400 \\
\hline & 4.85413200 & 1.84021500 & -0.07970300 \\
\hline & 3.15574800 & 2.95159100 & -1.27065900 \\
\hline & 4.64108100 & 4.05791600 & -0.29824400 \\
\hline & 4.41140000 & 1.23164300 & 1.15205800 \\
\hline & 3.33135600 & 1.93489900 & 3.08045500 \\
\hline & 4.50182200 & 1.17618400 & -1.30147800 \\
\hline & 5.94019900 & 2.01104300 & -0.04469200 \\
\hline & 3.48996700 & 1.81902100 & -1.99663800 \\
\hline & 3.03402700 & 1.48900400 & -3.06481800 \\
\hline & 5.02358900 & 0.07004700 & 1.74464400 \\
\hline & 5.02574500 & -0.08617200 & -1.74291900 \\
\hline & 4.40882800 & -1.24567200 & -1.15116500 \\
\hline & 4.49592400 & -1.19049100 & 1.30252300 \\
\hline & 3.64936600 & -2.10895600 & -1.92861700 \\
\hline & 4.84766300 & -1.85573100 & 0.08122600 \\
\hline & 4.01192700 & -3.17448300 & 0.13877100 \\
\hline & 3.39213000 & -3.23016200 & -1.15528400 \\
\hline & 3.48139200 & -1.83017400 & 1.99666800 \\
\hline & 3.14417500 & -2.96159100 & 1.27023000 \\
\hline & 2.60895700 & -4.32615800 & -1.65803600 \\
\hline & 2.31265200 & -3.98057800 & 1.84891300 \\
\hline & 3.02554600 & -1.49881400 & 3.06447800 \\
\hline & 3.32861800 & -1.94507300 & -3.08086900 \\
\hline & 1.24346500 & -4.38075200 & -1.20769400 \\
\hline & 1.02703100 & -4.12853800 & 1.22295600 \\
\hline & 0.20102400 & -3.89105200 & -1.97930400 \\
\hline & 0.80140200 & -4.92752700 & 0.04268800 \\
\hline & -0.75160900 & -4.91550000 & -0.08851500 \\
\hline & -0.96747600 & -4.09852700 & -1.25873700 \\
\hline & 0.28798500 & -3.42887900 & -3.09121300 \\
\hline & -2.25170000 & -3.93986700 & -1.88740500 \\
\hline & -1.18732100 & -4.37310000 & 1.16495000 \\
\hline & -0.13827000 & -3.90306000 & 1.94069300 \\
\hline & -0.22149300 & -3.44142700 & 3.05334700 \\
\hline & -2.55077100 & -4.32497000 & 1.62132200 \\
\hline & -3.08976600 & -2.93682600 & -1.29309500 \\
\hline & -3.33685700 & -3.22734000 & 1.12941800 \\
\hline & -3.37475500 & -1.76014800 & -1.96766400 \\
\hline & -3.95749800 & -3.16142600 & -0.16511000 \\
\hline & -4.79850300 & -1.84412200 & -0.09693600 \\
\hline & -4.38139700 & -1.12329900 & -1.26623400 \\
\hline & -2.88318900 & -1.39430900 & -3.00926000 \\
\hline
\end{tabular}




\begin{tabular}{|c|c|c|c|}
\hline $\mathrm{C}$ & -4.97010300 & 0.09853100 & -1.74274500 \\
\hline $\mathrm{N}$ & -4.43192500 & -1.29102900 & 1.18124900 \\
\hline $\mathrm{C}$ & -3.62780000 & -2.13275800 & 1.92855700 \\
\hline $\mathrm{O}$ & -3.29676200 & -1.97864500 & 3.08007000 \\
\hline $\mathrm{C}$ & -4.96829800 & -0.08298100 & 1.74274600 \\
\hline $\mathrm{N}$ & -4.42863100 & 1.30453400 & -1.18179600 \\
\hline $\mathrm{N}$ & -4.37552800 & 1.13653000 & 1.26558100 \\
\hline $\mathrm{C}$ & -4.79168800 & 1.85878100 & 0.09685800 \\
\hline $\mathrm{C}$ & -3.36783800 & 1.77138500 & 1.96735300 \\
\hline $\mathrm{C}$ & -3.94642000 & 3.17340200 & 0.16398600 \\
\hline $\mathrm{N}$ & -3.08056500 & 2.94756500 & 1.29296800 \\
\hline $\mathrm{C}$ & -3.62117700 & 2.14282900 & -1.92944900 \\
\hline $\mathrm{N}$ & -3.32446300 & 3.23566500 & -1.13016800 \\
\hline $\mathrm{O}$ & -2.87752000 & 1.40485200 & 3.00928500 \\
\hline $\mathrm{O}$ & -3.29167500 & 1.98742200 & -3.08124300 \\
\hline $\mathrm{C}$ & -2.23895500 & 3.94819500 & 1.88602100 \\
\hline $\mathrm{C}$ & -2.53597400 & 4.33110300 & -1.62309100 \\
\hline $\mathrm{H}$ & 2.84593600 & -4.94344500 & 1.81315800 \\
\hline $\mathrm{H}$ & 2.12831800 & -3.69592700 & 2.88852600 \\
\hline $\mathrm{H}$ & 2.57962200 & -4.21481900 & -2.74476100 \\
\hline $\mathrm{H}$ & 3.10275700 & -5.26904200 & -1.38983200 \\
\hline $\mathrm{H}$ & -2.78459200 & -4.90326800 & -1.87532400 \\
\hline $\mathrm{H}$ & -2.06115700 & -3.63303600 & -2.91950300 \\
\hline $\mathrm{H}$ & -3.04228500 & -5.26833200 & 1.34922200 \\
\hline $\mathrm{H}$ & -2.51758300 & -4.21978800 & 2.70861500 \\
\hline $\mathrm{H}$ & -6.05086500 & 0.05909800 & -1.54959900 \\
\hline $\mathrm{H}$ & -4.78496300 & 0.15069000 & -2.81868200 \\
\hline $\mathrm{H}$ & -6.04915000 & -0.03955600 & 1.55081800 \\
\hline $\mathrm{H}$ & -4.78219200 & -0.13591200 & 2.81848800 \\
\hline $\mathrm{H}$ & -2.50303200 & 4.22500600 & -2.71030600 \\
\hline $\mathrm{H}$ & -3.02524000 & 5.27585000 & -1.35169600 \\
\hline $\mathrm{H}$ & -2.04944200 & 3.64197100 & 2.91848300 \\
\hline $\mathrm{H}$ & -2.76842500 & 4.91345800 & 1.87275900 \\
\hline $\mathrm{H}$ & 2.86163900 & 4.93403500 & -1.81377700 \\
\hline $\mathrm{H}$ & 2.14147800 & 3.68782600 & -2.88892800 \\
\hline $\mathrm{H}$ & 2.59325200 & 4.20838100 & 2.74413500 \\
\hline $\mathrm{H}$ & 3.12006200 & 5.25997400 & 1.38860500 \\
\hline $\mathrm{H}$ & 5.93320200 & -2.03023700 & 0.04780300 \\
\hline $\mathrm{H}$ & 4.62667300 & -4.07275400 & 0.29913500 \\
\hline $\mathrm{H}$ & -1.18641200 & -5.91538400 & -0.23749700 \\
\hline $\mathrm{H}$ & 1.22199900 & -5.93498400 & 0.18063300 \\
\hline $\mathrm{H}$ & -4.56849700 & -4.06041300 & -0.33442500 \\
\hline $\mathrm{H}$ & -5.88390200 & -2.01835000 & -0.13413600 \\
\hline $\mathrm{H}$ & -4.55455300 & 4.07468300 & 0.33150500 \\
\hline $\mathrm{H}$ & 6.10406000 & 0.08527400 & 1.54595900 \\
\hline $\mathrm{H}$ & 4.84044500 & 0.13421100 & 2.82031500 \\
\hline $\mathrm{H}$ & 4.84378600 & -0.14969100 & -2.81882800 \\
\hline $\mathrm{H}$ & 6.10589700 & -0.10514900 & -1.54284900 \\
\hline $\mathrm{H}$ & -5.87650100 & 2.03643900 & 0.13537400 \\
\hline $\mathrm{C}$ & -1.12428400 & -0.48738300 & 0.45461000 \\
\hline $\mathrm{H}$ & -1.11476900 & -0.29760100 & 1.52473200 \\
\hline $\mathrm{C}$ & -1.12240400 & 0.49597700 & -0.44037600 \\
\hline $\mathrm{H}$ & -1.10471900 & 1.53824900 & -0.12744700 \\
\hline $\mathrm{H}$ & -1.13588600 & 0.30661200 & -1.51058700 \\
\hline $\mathrm{H}$ & -1.13393000 & -1.53003700 & 0.14253700 \\
\hline
\end{tabular}

$\mathrm{C}_{2} \mathrm{H}_{6} @ \mathrm{CB}[6]$ 


\begin{tabular}{|c|c|c|c|}
\hline $\mathrm{C}$ & 5.03388200 & 0.79864600 & -0.11287700 \\
\hline $\mathrm{C}$ & 4.53934200 & 2.28275000 & -0.08288300 \\
\hline $\mathrm{N}$ & 3.84354700 & 2.37520400 & 1.17381500 \\
\hline $\mathrm{C}$ & 3.93809500 & 1.21914700 & 1.93130400 \\
\hline $\mathrm{N}$ & 4.58226300 & 0.27412100 & 1.14851200 \\
\hline $\mathrm{N}$ & 4.41784200 & 0.27233300 & -1.30121500 \\
\hline $\mathrm{H}$ & 6.12766000 & 0.70554400 & -0.18625400 \\
\hline $\mathrm{N}$ & 3.74350600 & 2.39234600 & -1.27403500 \\
\hline $\mathrm{H}$ & 5.35976000 & 3.01531400 & -0.11146600 \\
\hline $\mathrm{C}$ & 3.35183200 & 3.59863100 & 1.74737200 \\
\hline $\mathrm{O}$ & 3.58540800 & 1.08061700 & 3.07786100 \\
\hline C & 3.16890100 & 3.60934900 & -1.77252800 \\
\hline $\mathrm{C}$ & 3.68415200 & 1.21584100 & -2.00091600 \\
\hline $\mathrm{O}$ & 3.15108400 & 1.06001600 & -3.07330200 \\
\hline $\mathrm{N}$ & 1.91710200 & 3.98119700 & -1.16679500 \\
\hline $\mathrm{C}$ & 1.81228700 & 4.73407400 & 0.05982400 \\
\hline $\mathrm{C}$ & 0.76003000 & 4.05112500 & -1.93016900 \\
\hline $\mathrm{C}$ & 0.28890800 & 5.06313800 & 0.14121600 \\
\hline $\mathrm{N}$ & 2.06155400 & 4.02477300 & 1.28063400 \\
\hline $\mathrm{H}$ & 2.44923000 & 5.62989300 & 0.00917700 \\
\hline $\mathrm{N}$ & -0.20200300 & 4.67395200 & -1.15001700 \\
\hline $\mathrm{O}$ & 0.63245900 & 3.69531500 & -3.07677600 \\
\hline $\mathrm{N}$ & -0.15183000 & 4.28189900 & 1.27062100 \\
\hline $\mathrm{H}$ & 0.08293500 & 6.12965600 & 0.31585000 \\
\hline $\mathrm{C}$ & 0.90517800 & 3.74412900 & 1.99003100 \\
\hline $\mathrm{O}$ & 0.83857600 & 3.19361300 & 3.06273100 \\
\hline $\mathrm{C}$ & -1.54533400 & 4.87004100 & -1.62530200 \\
\hline $\mathrm{C}$ & -1.45195800 & 4.41103900 & 1.87138500 \\
\hline $\mathrm{N}$ & -2.46925000 & 3.59697400 & 1.26743300 \\
\hline $\mathrm{N}$ & -2.52004300 & 3.91760600 & -1.16376700 \\
\hline $\mathrm{C}$ & -3.07213300 & 2.57200600 & 1.97844500 \\
\hline $\mathrm{C}$ & -3.21453600 & 3.97246000 & 0.09168400 \\
\hline $\mathrm{C}$ & -4.27097200 & 2.82843400 & -0.02903400 \\
\hline $\mathrm{N}$ & -4.13582900 & 2.12008500 & 1.21233300 \\
\hline $\mathrm{C}$ & -2.90375300 & 2.83896000 & -1.94558100 \\
\hline $\mathrm{N}$ & -3.85793900 & 2.13778600 & -1.22569400 \\
\hline $\mathrm{C}$ & -4.98526900 & 1.05113300 & 1.66550600 \\
\hline $\mathrm{C}$ & -4.59812400 & 1.06844600 & -1.83594700 \\
\hline $\mathrm{O}$ & -2.52417900 & 2.59463200 & -3.06550100 \\
\hline $\mathrm{O}$ & -2.76958600 & 2.18682300 & 3.08218000 \\
\hline $\mathrm{N}$ & -4.66936400 & -0.25733700 & 1.15857400 \\
\hline $\mathrm{N}$ & -4.34442800 & -0.22859000 & -1.27076100 \\
\hline $\mathrm{C}$ & -3.98083700 & -1.18516200 & 1.92413800 \\
\hline $\mathrm{C}$ & -5.04756500 & -0.76131700 & -0.13133500 \\
\hline $\mathrm{C}$ & -4.57789400 & -2.24980700 & -0.08726900 \\
\hline $\mathrm{N}$ & -3.82308700 & -2.32101500 & 1.14084500 \\
\hline $\mathrm{O}$ & -3.64134200 & -1.05401200 & 3.07513200 \\
\hline $\mathrm{C}$ & -3.37767300 & -3.55575400 & 1.73504400 \\
\hline $\mathrm{N}$ & -3.84640300 & -2.39913800 & -1.31222000 \\
\hline $\mathrm{C}$ & -3.69917100 & -1.20991000 & -2.00745700 \\
\hline $\mathrm{O}$ & -3.16669200 & -1.06906200 & -3.08197600 \\
\hline $\mathrm{C}$ & -3.24816700 & -3.61775400 & -1.77779100 \\
\hline $\mathrm{N}$ & -2.09929400 & -4.02509000 & 1.28180600 \\
\hline $\mathrm{N}$ & -1.98736400 & -3.95277200 & -1.16794200 \\
\hline $\mathrm{C}$ & -0.94287000 & -3.78357000 & 2.00446400 \\
\hline $\mathrm{C}$ & -1.85991400 & -4.71790900 & 0.04818400 \\
\hline $\mathrm{C}$ & -0.33225800 & -5.04162700 & 0.10838600 \\
\hline $\mathrm{N}$ & 0.10619800 & -4.32838400 & 1.28102300 \\
\hline $\mathrm{O}$ & -0.87114700 & -3.25724400 & 3.08887700 \\
\hline $\mathrm{C}$ & 1.42183100 & -4.42768200 & 1.84831400 \\
\hline $\mathrm{N}$ & 0.15416100 & -4.57325100 & -1.15898200 \\
\hline
\end{tabular}




\begin{tabular}{|c|c|c|c|}
\hline C & -0.82551500 & -3.96456600 & -1.92612300 \\
\hline $\mathrm{O}$ & -0.70580700 & -3.57697200 & -3.06352200 \\
\hline C & 1.48476700 & -4.77007200 & -1.66678700 \\
\hline $\mathrm{N}$ & 2.40415300 & -3.57124000 & 1.24003600 \\
\hline $\mathrm{N}$ & 2.47753400 & -3.84419200 & -1.19441900 \\
\hline C & 3.15943300 & -3.92901200 & 0.06484400 \\
\hline $\mathrm{C}$ & 2.87338900 & -2.75413800 & -1.95275900 \\
\hline C & 4.22610300 & -2.79411300 & -0.02798600 \\
\hline $\mathrm{N}$ & 3.83557800 & -2.07741300 & -1.21733600 \\
\hline C & 3.00637000 & -2.55676500 & 1.96980200 \\
\hline $\mathrm{N}$ & 4.08389300 & -2.10643600 & 1.22265000 \\
\hline $\mathrm{O}$ & 2.49912600 & -2.48338700 & -3.06844100 \\
\hline $\mathrm{O}$ & 2.69319000 & -2.18197300 & 3.07382300 \\
\hline C & 4.63149500 & -1.04523500 & -1.82767100 \\
\hline $\mathrm{C}$ & 4.91277100 & -1.02273000 & 1.67500500 \\
\hline $\mathrm{H}$ & -5.67502400 & 1.28502000 & -1.76585200 \\
\hline $\mathrm{H}$ & -4.29256400 & 1.02931100 & -2.88524000 \\
\hline $\mathrm{H}$ & -4.87431200 & 0.99713500 & 2.75138500 \\
\hline $\mathrm{H}$ & -6.02402500 & 1.29249400 & 1.40533600 \\
\hline $\mathrm{H}$ & -4.12631300 & -4.33889100 & 1.54891500 \\
\hline $\mathrm{H}$ & -3.28601000 & -3.37368700 & 2.80898300 \\
\hline $\mathrm{H}$ & -3.95955100 & -4.44036500 & -1.61890300 \\
\hline $\mathrm{H}$ & -3.05758900 & -3.48861200 & -2.84634500 \\
\hline $\mathrm{H}$ & 1.76372300 & -5.47215300 & 1.78369300 \\
\hline $\mathrm{H}$ & 1.34054600 & -4.12546100 & 2.89606600 \\
\hline $\mathrm{H}$ & 1.81022900 & -5.79006500 & -1.42340500 \\
\hline $\mathrm{H}$ & 1.42627700 & -4.64328300 & -2.75076000 \\
\hline $\mathrm{H}$ & 4.78654200 & -0.95942600 & 2.75878100 \\
\hline $\mathrm{H}$ & 5.95815400 & -1.25396300 & 1.43093900 \\
\hline $\mathrm{H}$ & 4.35501200 & -1.01489000 & -2.88509600 \\
\hline $\mathrm{H}$ & 5.69637000 & -1.30031400 & -1.72396800 \\
\hline $\mathrm{H}$ & 4.07513000 & 4.40332200 & 1.55678300 \\
\hline $\mathrm{H}$ & 3.25944600 & 3.42482100 & 2.82244100 \\
\hline $\mathrm{H}$ & 2.96952900 & 3.45760200 & -2.83672300 \\
\hline $\mathrm{H}$ & 3.89603900 & 4.42400500 & -1.63908100 \\
\hline $\mathrm{H}$ & -3.66412000 & 4.96723300 & 0.22865100 \\
\hline $\mathrm{H}$ & -5.30128800 & 3.19748200 & -0.14070000 \\
\hline $\mathrm{H}$ & -5.41060200 & -2.96829200 & -0.05767000 \\
\hline $\mathrm{H}$ & -6.13297500 & -0.65099200 & -0.27386900 \\
\hline $\mathrm{H}$ & -2.49214100 & -5.61681900 & -0.00541900 \\
\hline $\mathrm{H}$ & -0.11918300 & -6.11523900 & 0.21958400 \\
\hline $\mathrm{H}$ & 5.25404600 & -3.17110500 & -0.13554800 \\
\hline $\mathrm{H}$ & -1.87741700 & 5.87758700 & -1.34378600 \\
\hline $\mathrm{H}$ & -1.50506000 & 4.77856600 & -2.71359500 \\
\hline $\mathrm{H}$ & -1.36031800 & 4.08460400 & 2.91105000 \\
\hline $\mathrm{H}$ & -1.76157200 & 5.46706700 & 1.83520400 \\
\hline $\mathrm{H}$ & 3.59917400 & -4.93009800 & 0.18813300 \\
\hline $\mathrm{C}$ & 0.10793500 & -0.95229600 & -0.36763100 \\
\hline $\mathrm{H}$ & 0.22691100 & -1.07145900 & -1.44891300 \\
\hline $\mathrm{C}$ & 1.07964800 & 0.08575300 & 0.19107200 \\
\hline $\mathrm{H}$ & 2.11404700 & -0.23019600 & 0.02210100 \\
\hline $\mathrm{H}$ & 0.95033500 & 1.05822300 & -0.29660900 \\
\hline $\mathrm{H}$ & -0.93279100 & -0.67219400 & -0.17425200 \\
\hline $\mathrm{H}$ & 0.94680500 & 0.22547700 & 1.26821600 \\
\hline $\mathrm{H}$ & 0.27832000 & -1.92780100 & 0.09993900 \\
\hline
\end{tabular}




\begin{tabular}{|c|c|c|c|}
\hline $\mathrm{C}$ & 5.10864800 & -0.76575800 & 0.13936500 \\
\hline $\mathrm{C}$ & 5.10690800 & 0.79795300 & 0.12023300 \\
\hline $\mathrm{N}$ & 4.38829800 & 1.14166700 & 1.31513000 \\
\hline $\mathrm{C}$ & 3.92163200 & 0.03723400 & 2.00867400 \\
\hline $\mathrm{N}$ & 4.29303500 & -1.08320700 & 1.28249800 \\
\hline $\mathrm{N}$ & 4.58289500 & -1.11298800 & -1.15257900 \\
\hline $\mathrm{H}$ & 6.11266100 & -1.19822600 & 0.26374600 \\
\hline $\mathrm{N}$ & 4.47460600 & 1.11271200 & -1.13600800 \\
\hline $\mathrm{H}$ & 6.11624000 & 1.23436800 & 0.15178200 \\
\hline $\mathrm{C}$ & 4.18283800 & 2.47692900 & 1.80194400 \\
\hline $\mathrm{O}$ & 3.35448800 & 0.04673600 & 3.07477000 \\
\hline $\mathrm{C}$ & 4.39623300 & 2.43450700 & -1.70078300 \\
\hline $\mathrm{C}$ & 4.25843400 & -0.00820500 & -1.92376400 \\
\hline $\mathrm{O}$ & 3.91481300 & -0.01721700 & -3.08109600 \\
\hline $\mathrm{N}$ & 3.27992200 & 3.22538900 & -1.26090300 \\
\hline $\mathrm{C}$ & 3.21221100 & 3.95546700 & -0.02812400 \\
\hline $\mathrm{C}$ & 2.09750600 & 3.27205100 & -1.98316500 \\
\hline $\mathrm{C}$ & 1.83499600 & 4.68450500 & -0.12034600 \\
\hline $\mathrm{N}$ & 3.09012500 & 3.18603600 & 1.18761800 \\
\hline $\mathrm{H}$ & 4.06522500 & 4.64622700 & 0.04586800 \\
\hline $\mathrm{N}$ & 1.21273500 & 4.05895800 & -1.26139900 \\
\hline $\mathrm{O}$ & 1.89991400 & 2.77378800 & -3.06511700 \\
\hline $\mathrm{N}$ & 1.24099300 & 4.43290600 & 1.16157100 \\
\hline $\mathrm{H}$ & 1.92771500 & 5.76834700 & -0.28578300 \\
\hline $\mathrm{C}$ & 1.98820600 & 3.56528400 & 1.94243100 \\
\hline $\mathrm{O}$ & 1.75641100 & 3.25040300 & 3.08472900 \\
\hline $\mathrm{C}$ & -0.00391600 & 4.52862700 & -1.86610200 \\
\hline $\mathrm{C}$ & 0.02155900 & 5.02225700 & 1.64633500 \\
\hline $\mathrm{N}$ & -1.19684900 & 4.41704200 & 1.17978700 \\
\hline $\mathrm{N}$ & -1.20553300 & 4.04427100 & -1.24365800 \\
\hline $\mathrm{C}$ & -1.92552600 & 3.54324600 & 1.97207900 \\
\hline $\mathrm{C}$ & -1.81336100 & 4.66471700 & -0.09236300 \\
\hline $\mathrm{C}$ & -3.18347400 & 3.92688900 & 0.02008900 \\
\hline $\mathrm{N}$ & -3.03936200 & 3.16518600 & 1.23624800 \\
\hline $\mathrm{C}$ & -2.09745500 & 3.25401300 & -1.95402700 \\
\hline $\mathrm{N}$ & -3.26244400 & 3.18647100 & -1.20538100 \\
\hline $\mathrm{C}$ & -4.10324800 & 2.41092000 & 1.84247700 \\
\hline $\mathrm{C}$ & -4.40953800 & 2.44571600 & -1.65702900 \\
\hline $\mathrm{O}$ & -1.91821400 & 2.76844300 & -3.04481900 \\
\hline $\mathrm{O}$ & -1.67103200 & 3.22381100 & 3.10815100 \\
\hline $\mathrm{N}$ & -4.28214200 & 1.08972300 & 1.30784000 \\
\hline $\mathrm{N}$ & -4.53392500 & 1.11254700 & -1.13404800 \\
\hline $\mathrm{C}$ & -3.86171200 & -0.02824200 & 2.00892000 \\
\hline $\mathrm{C}$ & -5.07688500 & 0.76705900 & 0.15265300 \\
\hline $\mathrm{C}$ & -5.06899700 & -0.79829200 & 0.13760100 \\
\hline $\mathrm{N}$ & -4.29960800 & -1.13659100 & 1.30393400 \\
\hline $\mathrm{O}$ & -3.28087500 & -0.03496200 & 3.06763300 \\
\hline $\mathrm{C}$ & -4.11125600 & -2.46596300 & 1.81329800 \\
\hline $\mathrm{N}$ & -4.49057700 & -1.11293500 & -1.14228800 \\
\hline $\mathrm{C}$ & -4.23776900 & 0.00788300 & -1.91464300 \\
\hline $\mathrm{O}$ & -3.88737500 & 0.01903900 & -3.07077100 \\
\hline $\mathrm{C}$ & -4.36204500 & -2.43492500 & -1.69193500 \\
\hline $\mathrm{N}$ & -3.03184100 & -3.19737400 & 1.20488400 \\
\hline $\mathrm{N}$ & -3.22221700 & -3.18588400 & -1.24016400 \\
\hline $\mathrm{C}$ & -1.93084100 & -3.59049400 & 1.95302600 \\
\hline $\mathrm{C}$ & -3.15710800 & -3.94256400 & -0.02422500 \\
\hline $\mathrm{C}$ & -1.78319600 & -4.67399100 & -0.12943000 \\
\hline $\mathrm{N}$ & -1.18726500 & -4.44730300 & 1.15623100 \\
\hline $\mathrm{O}$ & -1.69698000 & -3.29391000 & 3.09965700 \\
\hline $\mathrm{C}$ & 0.03267900 & -5.04714300 & 1.62604700 \\
\hline $\mathrm{N}$ & -1.15971700 & -4.03094700 & -1.26010500 \\
\hline
\end{tabular}




\begin{tabular}{|c|c|c|c|}
\hline C & -2.04460100 & -3.23148900 & -1.96913500 \\
\hline $\mathrm{O}$ & -1.84948800 & -2.72224800 & -3.04671200 \\
\hline C & 0.04753900 & -4.50773200 & -1.87846700 \\
\hline $\mathrm{N}$ & 1.25101300 & -4.43962400 & 1.16181600 \\
\hline $\mathrm{N}$ & 1.25858100 & -4.04430200 & -1.25872800 \\
\hline C & 1.86696500 & -4.67560500 & -0.11289400 \\
\hline C & 2.15235700 & -3.25498700 & -1.96690700 \\
\hline $\mathrm{C}$ & 3.23695200 & -3.93667800 & 0.00496100 \\
\hline $\mathrm{N}$ & 3.31872000 & -3.19375800 & -1.21985000 \\
\hline C & 1.97203600 & -3.55943700 & 1.95424700 \\
\hline $\mathrm{N}$ & 3.08674700 & -3.17900800 & 1.22306800 \\
\hline $\mathrm{O}$ & 1.97333700 & -2.76465100 & -3.05568700 \\
\hline $\mathrm{O}$ & 1.71039900 & -3.23725800 & 3.08814100 \\
\hline C & 4.46397300 & -2.44899700 & -1.67144800 \\
\hline C & 4.13587400 & -2.40441000 & 1.82712700 \\
\hline $\mathrm{H}$ & -5.31371500 & 3.01711900 & -1.40847200 \\
\hline $\mathrm{H}$ & -4.31625800 & 2.34380600 & -2.74114600 \\
\hline $\mathrm{H}$ & -3.84747900 & 2.29883200 & 2.89956100 \\
\hline $\mathrm{H}$ & -5.04851900 & 2.96414500 & 1.74121700 \\
\hline $\mathrm{H}$ & -5.04962500 & -3.02615300 & 1.69007100 \\
\hline $\mathrm{H}$ & -3.86855900 & -2.37307000 & 2.87523600 \\
\hline $\mathrm{H}$ & -5.26968100 & -3.01116800 & -1.46719900 \\
\hline $\mathrm{H}$ & -4.25487300 & -2.31045400 & -2.77240600 \\
\hline $\mathrm{H}$ & 0.03765100 & -6.10749100 & 1.34123900 \\
\hline $\mathrm{H}$ & 0.02826500 & -4.95159900 & 2.71471100 \\
\hline $\mathrm{H}$ & 0.04046700 & -5.60923500 & -1.88338900 \\
\hline $\mathrm{H}$ & 0.05164400 & -4.13089000 & -2.90513000 \\
\hline $\mathrm{H}$ & 3.87613300 & -2.28978800 & 2.88304400 \\
\hline $\mathrm{H}$ & 5.09030800 & -2.94337800 & 1.73161400 \\
\hline $\mathrm{H}$ & 4.37326600 & -2.35153700 & -2.75615800 \\
\hline $\mathrm{H}$ & 5.37007900 & -3.01483500 & -1.41851600 \\
\hline $\mathrm{H}$ & 5.11269200 & 3.04716500 & 1.66420200 \\
\hline $\mathrm{H}$ & 3.94803700 & 2.39685500 & 2.86662300 \\
\hline $\mathrm{H}$ & 4.28912000 & 2.30583000 & -2.78085000 \\
\hline $\mathrm{H}$ & 5.32210700 & 2.98156800 & -1.47687500 \\
\hline $\mathrm{H}$ & -1.91543700 & 5.74816000 & -0.25562200 \\
\hline $\mathrm{H}$ & -4.04109500 & 4.61144700 & 0.10067300 \\
\hline $\mathrm{H}$ & -6.07426600 & -1.23871300 & 0.21335200 \\
\hline $\mathrm{H}$ & -6.08417300 & 1.19585300 & 0.26109900 \\
\hline $\mathrm{H}$ & -4.01328600 & -4.63102100 & 0.03572200 \\
\hline $\mathrm{H}$ & -1.87932700 & -5.75459200 & -0.31357800 \\
\hline $\mathrm{H}$ & 4.09490400 & -4.62052400 & 0.08605200 \\
\hline $\mathrm{H}$ & -0.01057800 & 5.63011100 & -1.85833100 \\
\hline $\mathrm{H}$ & -0.00937400 & 4.16339200 & -2.89694800 \\
\hline $\mathrm{H}$ & 0.03110800 & 4.91145100 & 2.73358000 \\
\hline $\mathrm{H}$ & 0.01272800 & 6.08653900 & 1.37637300 \\
\hline 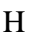 & 1.97014300 & -5.75731700 & -0.2857570 \\
\hline
\end{tabular}

\section{$\mathrm{Cl}_{2} @ \mathrm{CB}[6]$}

$\begin{array}{lrrr}\mathrm{C} & 4.44992400 & 2.43829600 & 0.09828800 \\ \mathrm{C} & 3.47265300 & 3.65641600 & 0.14316200 \\ \mathrm{~N} & 2.63990800 & 3.36839200 & 1.28332100 \\ \mathrm{C} & 3.08527600 & 2.27533500 & 2.01048200 \\ \mathrm{~N} & 4.14716700 & 1.72927200 & 1.30842200 \\ \mathrm{~N} & 4.11213100 & 1.78987300 & -1.14448200 \\ \mathrm{H} & 5.51044000 & 2.73149600 & 0.09146600\end{array}$




\begin{tabular}{|c|c|c|c|}
\hline $\mathrm{N}$ & 2.84207300 & 3.62092100 & -1.14647600 \\
\hline $\mathrm{H}$ & 3.98366700 & 4.62053000 & 0.28441600 \\
\hline $\mathrm{C}$ & 1.70638900 & 4.30131300 & 1.85248900 \\
\hline $\mathrm{O}$ & 2.67071200 & 1.90766300 & 3.08349000 \\
\hline C & 1.94090800 & 4.61713100 & -1.65844100 \\
\hline $\mathrm{C}$ & 3.23860100 & 2.54032600 & -1.91678300 \\
\hline $\mathrm{O}$ & 2.93214200 & 2.32843300 & -3.06578900 \\
\hline $\mathrm{N}$ & 0.58098600 & 4.52810300 & -1.19962600 \\
\hline C & 0.08394800 & 5.04541600 & 0.04271400 \\
\hline C & -0.40100400 & 3.90978700 & -1.95703700 \\
\hline C & -1.45997100 & 4.85177100 & -0.07789300 \\
\hline $\mathrm{N}$ & 0.40842300 & 4.30072700 & 1.23493100 \\
\hline $\mathrm{H}$ & 0.38348700 & 6.09757200 & 0.15900100 \\
\hline $\mathrm{N}$ & -1.58732400 & 4.01866700 & -1.24865000 \\
\hline $\mathrm{O}$ & -0.25995500 & 3.42509000 & -3.05442600 \\
\hline $\mathrm{N}$ & -1.81926500 & 4.26369900 & 1.18074100 \\
\hline $\mathrm{H}$ & -2.00677500 & 5.79539400 & -0.22199600 \\
\hline C & -0.71767200 & 3.93663400 & 1.95731600 \\
\hline $\mathrm{O}$ & -0.73964600 & 3.48111500 & 3.07533900 \\
\hline C & -2.84223600 & 3.68927500 & -1.86634600 \\
\hline C & -3.16153000 & 4.01413800 & 1.63425900 \\
\hline $\mathrm{N}$ & -3.77642500 & 2.80940600 & 1.14442600 \\
\hline $\mathrm{N}$ & -3.51739900 & 2.56369300 & -1.28170800 \\
\hline C & -3.84690300 & 1.66450200 & 1.92259000 \\
\hline C & -4.39520200 & 2.65293000 & -0.14182800 \\
\hline C & -5.02310700 & 1.22531000 & -0.06701700 \\
\hline $\mathrm{N}$ & -4.47496700 & 0.69128600 & 1.15700100 \\
\hline $\mathrm{C}$ & -3.69625200 & 1.39342600 & -2.00267600 \\
\hline $\mathrm{N}$ & -4.59848000 & 0.61580900 & -1.29425300 \\
\hline $\mathrm{C}$ & -4.91741200 & -0.53698400 & 1.76545700 \\
\hline C & -4.95201800 & -0.70498200 & -1.73376700 \\
\hline $\mathrm{O}$ & -3.21014700 & 1.12808000 & -3.07571900 \\
\hline $\mathrm{O}$ & -3.48881900 & 1.55498800 & 3.07025300 \\
\hline $\mathrm{N}$ & -4.24348200 & -1.72006700 & 1.31024400 \\
\hline $\mathrm{N}$ & -4.18733000 & -1.77312900 & -1.14284900 \\
\hline $\mathrm{C}$ & -3.16722400 & -2.25231100 & 2.00140100 \\
\hline C & -4.53755900 & -2.42509600 & 0.09564500 \\
\hline C & -3.56268100 & -3.64469600 & 0.14390800 \\
\hline $\mathrm{N}$ & -2.72463700 & -3.34854400 & 1.27772800 \\
\hline $\mathrm{O}$ & -2.73950900 & -1.87215800 & 3.06478300 \\
\hline $\mathrm{C}$ & -1.78884700 & -4.27735000 & 1.84865300 \\
\hline $\mathrm{N}$ & -2.93795000 & -3.61991600 & -1.14923600 \\
\hline C & -3.33485300 & -2.54154600 & -1.92375700 \\
\hline $\mathrm{O}$ & -3.04495200 & -2.34383900 & -3.07892100 \\
\hline C & -2.03729700 & -4.61927900 & -1.65829400 \\
\hline $\mathrm{N}$ & -0.49394000 & -4.28118500 & 1.22621900 \\
\hline $\mathrm{N}$ & -0.67482000 & -4.52539000 & -1.20620100 \\
\hline C & 0.63232500 & -3.90041700 & 1.93738600 \\
\hline C & -0.17219200 & -5.03314800 & 0.03801300 \\
\hline C & 1.37047800 & -4.83522900 & -0.08807400 \\
\hline $\mathrm{N}$ & 1.73242700 & -4.24689800 & 1.16929300 \\
\hline $\mathrm{O}$ & 0.65505900 & -3.41623000 & 3.04427100 \\
\hline C & 3.07504100 & -4.00265600 & 1.62391000 \\
\hline $\mathrm{N}$ & 1.49055700 & -3.99982200 & -1.25839300 \\
\hline $\mathrm{C}$ & 0.30462100 & -3.91093300 & -1.97164600 \\
\hline $\mathrm{O}$ & 0.16234700 & -3.44447800 & -3.07623200 \\
\hline $\mathrm{C}$ & 2.74349300 & -3.66278800 & -1.87618600 \\
\hline $\mathrm{N}$ & 3.69469300 & -2.79973600 & 1.13879000 \\
\hline $\mathrm{N}$ & 3.41454300 & -2.53747700 & -1.28443200 \\
\hline $\mathrm{C}$ & 4.30164300 & -2.63675600 & -0.15110200 \\
\hline $\mathrm{C}$ & 3.61865300 & -1.37663100 & -2.01472000 \\
\hline
\end{tabular}




\begin{tabular}{cccc}
$\mathrm{C}$ & 4.93376500 & -1.21214600 & -0.07123700 \\
$\mathrm{~N}$ & 4.51598300 & -0.60049000 & -1.29916000 \\
$\mathrm{C}$ & 3.75859800 & -1.65467800 & 1.91631300 \\
$\mathrm{~N}$ & 4.38374000 & -0.67960000 & 1.15262500 \\
$\mathrm{O}$ & 3.15367100 & -1.11712700 & -3.09884600 \\
$\mathrm{O}$ & 3.39738200 & -1.54693300 & 3.06375400 \\
$\mathrm{C}$ & 4.87474600 & 0.72018600 & -1.73422800 \\
$\mathrm{C}$ & 4.82268200 & 0.54814500 & 1.76435300 \\
$\mathrm{H}$ & -6.02002000 & -0.86633100 & -1.53212900 \\
$\mathrm{H}$ & -4.76547600 & -0.74369800 & -2.81004900 \\
$\mathrm{H}$ & -4.71802300 & -0.44598700 & 2.83629600 \\
$\mathrm{H}$ & -5.99567300 & -0.66213300 & 1.59362800 \\
$\mathrm{H}$ & -2.21747900 & -5.29041100 & 1.80230800 \\
$\mathrm{H}$ & -1.63752700 & -3.98398300 & 2.89105000 \\
$\mathrm{H}$ & -2.42425000 & -5.61307400 & -1.39810700 \\
$\mathrm{H}$ & -2.02002100 & -4.49598200 & -2.74410400 \\
$\mathrm{H}$ & 3.69962700 & -4.86113400 & 1.34529800 \\
$\mathrm{H}$ & 3.02745000 & -3.91029700 & 2.71170000 \\
$\mathrm{H}$ & 3.41112800 & -4.53827400 & -1.84653200 \\
$\mathrm{H}$ & 2.52496800 & -3.39712600 & -2.91430100 \\
$\mathrm{H}$ & 4.62038400 & 0.45546200 & 2.83452900 \\
$\mathrm{H}$ & 5.90143400 & 0.67476100 & 1.59562200 \\
$\mathrm{H}$ & 4.69220000 & 0.76082600 & -2.81107300 \\
$\mathrm{H}$ & 5.94211400 & 0.87916500 & -1.52818400 \\
$\mathrm{H}$ & 2.13526600 & 5.31391700 & 1.79819100 \\
$\mathrm{H}$ & 1.55886800 & 4.01469600 & 2.89736700 \\
$\mathrm{H}$ & 1.91834400 & 4.48628600 & -2.74324300 \\
$\mathrm{H}$ & 2.33001100 & 5.61239300 & -1.40693100 \\
$\mathrm{H}$ & -5.14008600 & 3.44740800 & -0.29884900 \\
$\mathrm{H}$ & -6.12182800 & 1.23605100 & -0.01339300 \\
$\mathrm{H}$ & -4.07475900 & -4.60678500 & 0.29465500 \\
$\mathrm{H}$ & -5.59839400 & -2.71659500 & 0.07911700 \\
$\mathrm{H}$ & -0.46821700 & -6.08528600 & 0.16256700 \\
$\mathrm{H}$ & 1.91963700 & -5.77703700 & -0.23474400 \\
$\mathrm{H}$ & 6.03234600 & -1.22691500 & -0.01389100 \\
$\mathrm{H}$ & -3.50635200 & 4.56683400 & -1.82945800 \\
$\mathrm{H}$ & -2.62645300 & 3.42971500 & -2.90651100 \\
$\mathrm{H}$ & -3.11295400 & 3.91920900 & 2.72190500 \\
$\mathrm{H}$ & -3.79009500 & 4.87061700 & 1.35803200 \\
\hline & 5.04325500 & -3.43195700 & -0.32007300 \\
$\mathrm{H}$ & 0.65771400 & -0.40293300 & 1.01194600 \\
$\mathrm{H}$ & & 0.18966300 & -0.91247500
\end{tabular}

\section{$\mathrm{F}_{2} @ \mathrm{CB}[6]$}

$\begin{array}{llcc}\mathrm{C} & 4.16509100 & -3.01316400 & 0.10223900 \\ \mathrm{C} & 4.92520500 & -1.64801700 & 0.05842100 \\ \mathrm{~N} & 4.54831300 & -1.02235700 & 1.29386500 \\ \mathrm{C} & 3.60481200 & -1.74595200 & 2.00554800 \\ \mathrm{~N} & 3.32895700 & -2.88664300 & 1.26879600 \\ \mathrm{~N} & 3.50212800 & -3.06395900 & -1.17112900 \\ \mathrm{H} & 4.83505600 & -3.87918300 & 0.21042600 \\ \mathrm{~N} & 4.44304300 & -1.04174800 & -1.15870200 \\ \mathrm{H} & 6.01838200 & -1.76184800 & 0.01271400 \\ \mathrm{C} & 5.00429500 & 0.26123400 & 1.74873900 \\ \mathrm{O} & 3.15719600 & -1.46590100 & 3.09156900 \\ \mathrm{C} & 5.00968400 & 0.14486200 & -1.74812600 \\ \mathrm{C} & 3.69743000 & -1.92178100 & -1.93125100\end{array}$




\begin{tabular}{|c|c|c|c|}
\hline $\mathrm{O}$ & 3.34017100 & -1.75028900 & -3.07163600 \\
\hline $\mathrm{N}$ & 4.44309800 & 1.38366700 & -1.29344300 \\
\hline $\mathrm{C}$ & 4.76511600 & 2.03907300 & -0.05796900 \\
\hline $\mathrm{C}$ & 3.44189500 & 2.02470600 & -2.00533100 \\
\hline C & 3.89221000 & 3.33507900 & -0.10117800 \\
\hline $\mathrm{N}$ & 4.33573800 & 1.39351700 & 1.15882800 \\
\hline $\mathrm{H}$ & 5.84497300 & 2.24397800 & -0.01183700 \\
\hline $\mathrm{N}$ & 3.07056200 & 3.13788800 & -1.26824700 \\
\hline $\mathrm{O}$ & 3.02028400 & 1.70784900 & -3.09159800 \\
\hline $\mathrm{N}$ & 3.22634700 & 3.32848900 & 1.17163700 \\
\hline $\mathrm{H}$ & 4.48453300 & 4.25596800 & -0.20917200 \\
\hline C & 3.52030400 & 2.20832500 & 1.93253100 \\
\hline $\mathrm{O}$ & 3.18176000 & 2.00888500 & 3.07409200 \\
\hline C & 2.21344500 & 4.14413100 & -1.83266400 \\
\hline C & 2.40465400 & 4.39185000 & 1.68480100 \\
\hline $\mathrm{N}$ & 1.04838800 & 4.41961300 & 1.20690100 \\
\hline $\mathrm{N}$ & 0.90966000 & 4.22665500 & -1.23302900 \\
\hline C & -0.00101300 & 3.92933000 & 1.96884300 \\
\hline C & 0.61886100 & 4.98372600 & -0.04128100 \\
\hline $\mathrm{C}$ & -0.93832900 & 4.91430900 & 0.04754400 \\
\hline $\mathrm{N}$ & -1.16152200 & 4.13129100 & 1.23745800 \\
\hline $\mathrm{C}$ & -0.22865300 & 3.92186000 & -1.96394500 \\
\hline $\mathrm{N}$ & -1.31660400 & 4.31745700 & -1.20160400 \\
\hline C & -2.45395100 & 3.94442000 & 1.83998700 \\
\hline C & -2.66540600 & 4.17640800 & -1.68088800 \\
\hline $\mathrm{O}$ & -0.26472700 & 3.45856100 & -3.07853100 \\
\hline $\mathrm{O}$ & 0.07660700 & 3.47098900 & 3.08336200 \\
\hline $\mathrm{N}$ & -3.23255000 & 2.87822200 & 1.27364700 \\
\hline $\mathrm{N}$ & -3.39721900 & 3.05160200 & -1.16471900 \\
\hline $\mathrm{C}$ & -3.50186000 & 1.73021100 & 1.99972600 \\
\hline C & -4.06553300 & 3.01015400 & 0.10613200 \\
\hline C & -4.83920600 & 1.65182400 & 0.06342300 \\
\hline $\mathrm{N}$ & -4.45422500 & 1.01655000 & 1.29168000 \\
\hline $\mathrm{O}$ & -3.04462000 & 1.43935800 & 3.07907700 \\
\hline C & -4.91914800 & -0.26344200 & 1.74771700 \\
\hline $\mathrm{N}$ & -4.37577200 & 1.04919200 & -1.16138900 \\
\hline C & -3.60351200 & 1.91171300 & -1.92442300 \\
\hline $\mathrm{O}$ & -3.23608000 & 1.73186100 & -3.06044900 \\
\hline C & -4.93172300 & -0.14472000 & -1.74316400 \\
\hline $\mathrm{N}$ & -4.25927000 & -1.40218200 & 1.16307800 \\
\hline $\mathrm{N}$ & -4.35188200 & -1.37778800 & -1.28899100 \\
\hline $\mathrm{C}$ & -3.42291500 & -2.20170100 & 1.92763100 \\
\hline $\mathrm{C}$ & -4.67594700 & -2.04305600 & -0.05938500 \\
\hline C & -3.79095600 & -3.33098400 & -0.10485600 \\
\hline $\mathrm{N}$ & -3.12486000 & -3.32092300 & 1.16750600 \\
\hline $\mathrm{O}$ & -3.07346000 & -1.99479800 & 3.06469500 \\
\hline $\mathrm{C}$ & -2.30368300 & -4.38320500 & 1.68222700 \\
\hline $\mathrm{N}$ & -2.96875300 & -3.12303300 & -1.26938700 \\
\hline C & -3.34029600 & -2.00455400 & -1.99751700 \\
\hline $\mathrm{O}$ & -2.91243300 & -1.67549200 & -3.07777600 \\
\hline C & -2.10891000 & -4.12307200 & -1.83966900 \\
\hline $\mathrm{N}$ & -0.94823300 & -4.41493500 & 1.20235400 \\
\hline $\mathrm{N}$ & -0.80684000 & -4.21223100 & -1.23623900 \\
\hline C & -0.52303500 & -4.97758700 & -0.04781100 \\
\hline C & 0.33421900 & -3.91926900 & -1.96741700 \\
\hline $\mathrm{C}$ & 1.03473200 & -4.92309200 & 0.04192000 \\
\hline $\mathrm{N}$ & 1.41887800 & -4.32602200 & -1.20551700 \\
\hline $\mathrm{C}$ & 0.10458200 & -3.93598000 & 1.96620100 \\
\hline $\mathrm{N}$ & 1.26365700 & -4.14659400 & 1.23488600 \\
\hline $\mathrm{O}$ & 0.37517200 & -3.45575400 & -3.08174600 \\
\hline $\mathrm{O}$ & 0.03096500 & -3.47991000 & 3.08193400 \\
\hline
\end{tabular}




$\begin{array}{lrrr}\mathrm{C} & 2.76924200 & -4.19099300 & -1.68246200 \\ \mathrm{C} & 2.55759500 & -3.96005700 & 1.83305400 \\ \mathrm{H} & -3.21804900 & 5.09683200 & -1.44940300 \\ \mathrm{H} & -2.60218100 & 4.03961000 & -2.76328800 \\ \mathrm{H} & -2.28117300 & 3.69326700 & 2.89007000 \\ \mathrm{H} & -3.02410200 & 4.88322400 & 1.76755800 \\ \mathrm{H} & -5.99866000 & -0.33437700 & 1.55616400 \\ \mathrm{H} & -4.72671700 & -0.30562600 & 2.82289000 \\ \mathrm{H} & -6.01234600 & -0.17311300 & -1.54728400 \\ \mathrm{H} & -4.74803000 & -0.08493500 & -2.81899300 \\ \mathrm{H} & -2.78147300 & -5.34454700 & 1.45101300 \\ \mathrm{H} & -2.25097200 & -4.24221800 & 2.76472800 \\ \mathrm{H} & -2.60565200 & -5.10318500 & -1.77216300 \\ \mathrm{H} & -1.95379100 & -3.85501800 & -2.88834500 \\ \mathrm{H} & 2.38909900 & -3.71588300 & 2.88550500 \\ \mathrm{H} & 3.13054800 & -4.89638000 & 1.75208700 \\ \mathrm{H} & 2.70964000 & -4.06043000 & -2.76583400 \\ \mathrm{H} & 3.31952700 & -5.11082800 & -1.44401400 \\ \mathrm{H} & 6.08371800 & 0.33947000 & 1.55882100 \\ \mathrm{H} & 4.80990100 & 0.30409300 & 2.82358000 \\ \mathrm{H} & 4.82004300 & 0.08447100 & -2.82297700 \\ \mathrm{H} & 6.09171100 & 0.16286200 & -1.55765900 \\ \mathrm{H} & 1.00559200 & 6.00894200 & -0.14231500 \\ \mathrm{H} & -1.41331700 & 5.90153200 & 0.15133900 \\ \mathrm{H} & -5.93171000 & 1.77517100 & 0.03081200 \\ \mathrm{H} & -4.72673800 & 3.88328000 & 0.21066800 \\ \mathrm{H} & -5.75431100 & -2.25706600 & -0.02155300 \\ \mathrm{H} & -4.37402100 & -4.25736600 & -0.21580000 \\ \mathrm{H} & 1.50069500 & -5.91488100 & 0.14262600 \\ \mathrm{H} & 2.70971800 & 5.12349500 & -1.75353900 \\ \mathrm{H} & 2.06298400 & 3.88611200 & -2.88452900 \\ \mathrm{H} & 2.35446100 & 4.25393200 & 2.76782700 \\ \mathrm{H} & 2.88057300 & 5.35304900 & 1.44979600 \\ \mathrm{H} & -0.91922300 & -5.99876000 & -0.15366500 \\ \mathrm{~F} & -1.40486300 & 0.03173800 & -0.71721400 \\ \mathrm{~F} & -1.41940400 & -0.01864300 & 0.67032800 \\ & & & \end{array}$

\section{$\mathrm{H}_{2} @ \mathrm{CB}[6]$}

$\begin{array}{lrrr}\mathrm{C} & -4.56053000 & 2.25613500 & 0.12975400 \\ \mathrm{C} & -5.04135300 & 0.77084500 & 0.07432100 \\ \mathrm{~N} & -4.53348700 & 0.21453100 & 1.29554200 \\ \mathrm{C} & -3.73898300 & 1.09827300 & 2.00818800 \\ \mathrm{~N} & -3.69443200 & 2.27794500 & 1.28094900 \\ \mathrm{~N} & -3.94281000 & 2.45187500 & -1.15199200 \\ \mathrm{H} & -5.38207500 & 2.97590400 & 0.26297000 \\ \mathrm{~N} & -4.46664000 & 0.28393100 & -1.15654300 \\ \mathrm{H} & -6.13641900 & 0.66892500 & 0.04224700 \\ \mathrm{C} & -4.76541600 & -1.12653700 & 1.75405300 \\ \mathrm{O} & -3.23520500 & 0.89898000 & 3.08728200 \\ \mathrm{C} & -4.80230500 & -0.98317800 & -1.75426400 \\ \mathrm{C} & -3.92358400 & 1.30301900 & -1.92704400 \\ \mathrm{O} & -3.56095200 & 1.21975600 & -3.07561000 \\ \mathrm{~N} & -4.02478200 & -2.10084100 & -1.29750400 \\ \mathrm{C} & -4.24308400 & -2.81798800 & -0.07402600 \\ \mathrm{C} & -2.92057500 & -2.55240400 & -2.00312400 \\ \mathrm{C} & -3.17673200 & -3.95755600 & -0.13259500\end{array}$




\begin{tabular}{|c|c|c|c|}
\hline $\mathrm{N}$ & -3.92512400 & -2.13219100 & 1.15539800 \\
\hline $\mathrm{H}$ & -5.27684800 & -3.19288700 & -0.03802800 \\
\hline $\mathrm{N}$ & -2.37535700 & -3.59893000 & -1.27551100 \\
\hline $\mathrm{O}$ & -2.54470600 & -2.15124600 & -3.07825300 \\
\hline $\mathrm{N}$ & -2.54347400 & -3.88331700 & 1.15413500 \\
\hline $\mathrm{H}$ & -3.61596300 & -4.95594700 & -0.27770500 \\
\hline $\mathrm{C}$ & -3.00905400 & -2.83231700 & 1.92879800 \\
\hline $\mathrm{O}$ & -2.71962000 & -2.60710400 & 3.07900300 \\
\hline C & -1.36763200 & -4.44853400 & -1.84932700 \\
\hline C & -1.56335000 & -4.81007300 & 1.65392300 \\
\hline $\mathrm{N}$ & -0.21441400 & -4.60349900 & 1.19735100 \\
\hline $\mathrm{N}$ & -0.07214800 & -4.33738300 & -1.23629300 \\
\hline C & 0.71387900 & -3.91837600 & 1.96544100 \\
\hline C & 0.32335000 & -5.06026800 & -0.05235100 \\
\hline $\mathrm{C}$ & 1.84408400 & -4.72707100 & 0.06496800 \\
\hline $\mathrm{N}$ & 1.89873700 & -3.89673500 & 1.24413300 \\
\hline C & 1.01288000 & -3.86396900 & -1.95750700 \\
\hline $\mathrm{N}$ & 2.14314000 & -4.09839200 & -1.18967600 \\
\hline C & 3.12062300 & -3.45184400 & 1.85680700 \\
\hline C & 3.45323000 & -3.71283000 & -1.64341800 \\
\hline $\mathrm{O}$ & 0.98753700 & -3.39240200 & -3.06889400 \\
\hline $\mathrm{O}$ & 0.54053700 & -3.47880700 & 3.07651500 \\
\hline $\mathrm{N}$ & 3.67475900 & -2.25738700 & 1.28069900 \\
\hline $\mathrm{N}$ & 3.94907600 & -2.45619700 & -1.14841100 \\
\hline C & 3.73342100 & -1.07864900 & 2.00968800 \\
\hline C & 4.55284300 & -2.24795600 & 0.13716800 \\
\hline C & 5.03273500 & -0.76335300 & 0.07297000 \\
\hline $\mathrm{N}$ & 4.53919300 & -0.20429000 & 1.29881500 \\
\hline $\mathrm{O}$ & 3.23043900 & -0.87473800 & 3.08821300 \\
\hline C & 4.77186700 & 1.13912800 & 1.75114800 \\
\hline $\mathrm{N}$ & 4.44262700 & -0.28073800 & -1.15247700 \\
\hline $\mathrm{C}$ & 3.90840000 & -1.30613500 & -1.92069100 \\
\hline $\mathrm{O}$ & 3.53650000 & -1.22578100 & -3.06647200 \\
\hline C & 4.77459800 & 0.98399500 & -1.75735000 \\
\hline $\mathrm{N}$ & 3.92695700 & 2.14242000 & 1.15566600 \\
\hline $\mathrm{N}$ & 4.00215800 & 2.10332800 & -1.29725900 \\
\hline C & 3.00477100 & 2.83485300 & 1.92859000 \\
\hline C & 4.23363900 & 2.82424400 & -0.07815000 \\
\hline C & 3.16935200 & 3.96659600 & -0.13009300 \\
\hline $\mathrm{N}$ & 2.52965100 & 3.88089200 & 1.15319700 \\
\hline $\mathrm{O}$ & 2.71751100 & 2.60765000 & 3.07896800 \\
\hline $\mathrm{C}$ & 1.55007400 & 4.80632300 & 1.65678800 \\
\hline $\mathrm{N}$ & 2.37243100 & 3.62191600 & -1.27963500 \\
\hline C & 2.90492500 & 2.56739900 & -2.00493200 \\
\hline $\mathrm{O}$ & 2.52504500 & 2.16962700 & -3.07993600 \\
\hline C & 1.36726300 & 4.47735300 & -1.84868500 \\
\hline $\mathrm{N}$ & 0.20223600 & 4.60372500 & 1.19547500 \\
\hline $\mathrm{N}$ & 0.06953800 & 4.36200400 & -1.24104800 \\
\hline $\mathrm{C}$ & -0.33173100 & 5.07235800 & -0.05167100 \\
\hline C & -1.00975600 & 3.87657600 & -1.96274800 \\
\hline C & -1.85376300 & 4.73772600 & 0.05745300 \\
\hline $\mathrm{N}$ & -2.14202200 & 4.09374100 & -1.19288900 \\
\hline C & -0.73396100 & 3.93029700 & 1.96489600 \\
\hline $\mathrm{N}$ & -1.91889700 & 3.92161700 & 1.24479400 \\
\hline $\mathrm{O}$ & -0.97941700 & 3.40974200 & -3.07602500 \\
\hline $\mathrm{O}$ & -0.56458300 & 3.49055800 & 3.07661300 \\
\hline $\mathrm{C}$ & -3.44898800 & 3.70657600 & -1.65330700 \\
\hline $\mathrm{C}$ & -3.14420400 & 3.47720500 & 1.85122800 \\
\hline $\mathrm{H}$ & 4.16414900 & -4.50424800 & -1.37368700 \\
\hline $\mathrm{H}$ & 3.39326400 & -3.61597400 & -2.73027100 \\
\hline $\mathrm{H}$ & 2.88907900 & -3.22792900 & 2.90184500 \\
\hline
\end{tabular}




$\begin{array}{lrrr}\mathrm{H} & 3.86913700 & -4.25739500 & 1.80364100 \\ \mathrm{H} & 5.82493100 & 1.39201800 & 1.56429800 \\ \mathrm{H} & 4.56903500 & 1.15385800 & 2.82514000 \\ \mathrm{H} & 5.83909600 & 1.19883700 & -1.58705400 \\ \mathrm{H} & 4.58114300 & 0.88047100 & -2.82815900 \\ \mathrm{H} & 1.85872400 & 5.82789300 & 1.39869800 \\ \mathrm{H} & 1.53415500 & 4.68283000 & 2.74261200 \\ \mathrm{H} & 1.70711400 & 5.52233000 & -1.78375200 \\ \mathrm{H} & 1.25270400 & 4.18677500 & -2.89660700 \\ \mathrm{H} & -2.91928700 & 3.26108800 & 2.89927500 \\ \mathrm{H} & -3.89345700 & 4.28104400 & 1.78732800 \\ \mathrm{H} & -3.38305900 & 3.60345700 & -2.73937600 \\ \mathrm{H} & -4.16245900 & 4.49879300 & -1.39193500 \\ \mathrm{H} & -5.82008200 & -1.37907600 & 1.57487600 \\ \mathrm{H} & -4.55553100 & -1.13840400 & 2.82677100 \\ \mathrm{H} & -4.61793900 & -0.88273500 & -2.82693500 \\ \mathrm{H} & -5.86483100 & -1.19878300 & -1.57435000 \\ \mathrm{H} & 0.12148200 & -6.13433900 & -0.17852900 \\ \mathrm{H} & 2.47614200 & -5.61739700 & 0.19923800 \\ \mathrm{H} & 6.12731200 & -0.66130600 & 0.02797500 \\ \mathrm{H} & 5.37327300 & -2.96571800 & 0.28671600 \\ \mathrm{H} & 5.26865800 & 3.19670400 & -0.05333000 \\ \mathrm{H} & 3.61102900 & 4.96556800 & -0.26340500 \\ \mathrm{H} & -2.48816200 & 5.62878500 & 0.17488400 \\ \mathrm{H} & -1.70687100 & -5.49460200 & -1.79566000 \\ \mathrm{H} & -1.25012800 & -4.14795400 & -2.89412500 \\ \mathrm{H} & -1.55027300 & -4.69320100 & 2.74046500 \\ \mathrm{H} & -1.86982500 & -5.83033000 & 1.38887600 \\ \mathrm{H} & -0.12991600 & 6.14764100 & -0.16714700 \\ \mathrm{H} & 1.04712300 & -1.07980800 & -0.81319100 \\ \mathrm{H} & 1.35088200 & -1.40012000 & -0.21364400\end{array}$

\section{$\mathrm{H}_{2} \mathrm{~S} @ \mathrm{CB}[6]$}

$\begin{array}{lrrr}\mathrm{C} & -4.74302900 & 0.11254300 & 1.83185800 \\ \mathrm{C} & -3.97829800 & 0.02612300 & 3.18999400 \\ \mathrm{~N} & -3.26986000 & 1.27142600 & 3.24967600 \\ \mathrm{C} & -3.37063200 & 2.00822200 & 2.07894000 \\ \mathrm{~N} & -4.16659600 & 1.27807100 & 1.20959400 \\ \mathrm{~N} & -4.47172000 & -1.15655300 & 1.21844500 \\ \mathrm{H} & -5.82855100 & 0.24613700 & 1.95220300 \\ \mathrm{~N} & -3.18858300 & -1.17282500 & 3.04095700 \\ \mathrm{H} & -4.64606400 & -0.06782100 & 4.05944700 \\ \mathrm{C} & -2.46965100 & 1.72080300 & 4.35476200 \\ \mathrm{O} & -2.90856600 & 3.10607600 & 1.88277400 \\ \mathrm{C} & -2.45424400 & -1.78702400 & 4.11356500 \\ \mathrm{C} & -3.56785700 & -1.92252700 & 1.93594000 \\ \mathrm{O} & -3.22874400 & -3.05413600 & 1.68579900 \\ \mathrm{~N} & -1.12631000 & -1.27598800 & 4.31396800 \\ \mathrm{C} & -0.79963500 & -0.07984200 & 5.04174100 \\ \mathrm{C} & -0.01696000 & -1.92232600 & 3.80569200 \\ \mathrm{C} & 0.76436100 & -0.07370900 & 5.03375500 \\ \mathrm{~N} & -1.13895300 & 1.17342800 & 4.41825400 \\ \mathrm{H} & -1.23124200 & -0.12864700 & 6.05213800 \\ \mathrm{~N} & 1.09509200 & -1.20373500 & 4.20335700 \\ \mathrm{O} & -0.01276100 & -2.95759200 & 3.17631200 \\ \mathrm{~N} & 1.08752000 & 1.22591900 & 4.51459300\end{array}$




\begin{tabular}{|c|c|c|c|}
\hline $\mathrm{H}$ & 1.20644300 & -0.20252300 & 6.03269900 \\
\hline C & -0.03090100 & 1.97712300 & 4.19016700 \\
\hline $\mathrm{O}$ & -0.04211300 & 3.13260000 & 3.84132600 \\
\hline $\mathrm{C}$ & 2.41812500 & -1.75499600 & 4.07172600 \\
\hline C & 2.41680500 & 1.75653100 & 4.36819100 \\
\hline $\mathrm{N}$ & 3.15634500 & 1.28381700 & 3.22869300 \\
\hline $\mathrm{N}$ & 3.21538700 & -1.15814800 & 3.04099900 \\
\hline C & 3.26289600 & 2.03541800 & 2.06771600 \\
\hline C & 3.93645700 & 0.08023700 & 3.18690900 \\
\hline C & 4.70877500 & 0.19157600 & 1.83428000 \\
\hline $\mathrm{N}$ & 4.09826600 & 1.33775400 & 1.20944900 \\
\hline C & 3.60928300 & -1.88534400 & 1.92977600 \\
\hline $\mathrm{N}$ & 4.48949300 & -1.08781500 & 1.21915500 \\
\hline C & 4.58001600 & 1.94556700 & 0.00000000 \\
\hline $\mathrm{C}$ & 5.10001000 & -1.54323500 & 0.00000000 \\
\hline $\mathrm{O}$ & 3.29968900 & -3.02503700 & 1.67312200 \\
\hline $\mathrm{O}$ & 2.77444000 & 3.12206000 & 1.87252800 \\
\hline $\mathrm{N}$ & 4.09826600 & 1.33775400 & -1.20944900 \\
\hline $\mathrm{N}$ & 4.48949300 & -1.08781500 & -1.21915500 \\
\hline $\mathrm{C}$ & 3.26289600 & 2.03541800 & -2.06771600 \\
\hline C & 4.70877500 & 0.19157600 & -1.83428000 \\
\hline C & 3.93645700 & 0.08023700 & -3.18690900 \\
\hline $\mathrm{N}$ & 3.15634500 & 1.28381700 & -3.22869300 \\
\hline $\mathrm{O}$ & 2.77444000 & 3.12206000 & -1.87252800 \\
\hline $\mathrm{C}$ & 2.41680500 & 1.75653100 & -4.36819100 \\
\hline $\mathrm{N}$ & 3.21538700 & -1.15814800 & -3.04099900 \\
\hline C & 3.60928300 & -1.88534400 & -1.92977600 \\
\hline $\mathrm{O}$ & 3.29968900 & -3.02503700 & -1.67312200 \\
\hline C & 2.41812500 & -1.75499600 & -4.07172600 \\
\hline $\mathrm{N}$ & 1.08752000 & 1.22591900 & -4.51459300 \\
\hline $\mathrm{N}$ & 1.09509200 & -1.20373500 & -4.20335700 \\
\hline $\mathrm{C}$ & -0.03090100 & 1.97712300 & -4.19016700 \\
\hline C & 0.76436100 & -0.07370900 & -5.03375500 \\
\hline C & -0.79963500 & -0.07984200 & -5.04174100 \\
\hline $\mathrm{N}$ & -1.13895300 & 1.17342800 & -4.41825400 \\
\hline $\mathrm{O}$ & -0.04211300 & 3.13260000 & -3.84132600 \\
\hline C & -2.46965100 & 1.72080300 & -4.35476200 \\
\hline $\mathrm{N}$ & -1.12631000 & -1.27598800 & -4.31396800 \\
\hline C & -0.01696000 & -1.92232600 & -3.80569200 \\
\hline $\mathrm{O}$ & -0.01276100 & -2.95759200 & -3.17631200 \\
\hline $\mathrm{C}$ & -2.45424400 & -1.78702400 & -4.11356500 \\
\hline $\mathrm{N}$ & -3.26986000 & 1.27142600 & -3.24967600 \\
\hline $\mathrm{N}$ & -3.18858300 & -1.17282500 & -3.04095700 \\
\hline C & -3.97829800 & 0.02612300 & -3.18999400 \\
\hline $\mathrm{C}$ & -3.56785700 & -1.92252700 & -1.93594000 \\
\hline $\mathrm{C}$ & -4.74302900 & 0.11254300 & -1.83185800 \\
\hline $\mathrm{N}$ & -4.47172000 & -1.15655300 & -1.21844500 \\
\hline C & -3.37063200 & 2.00822200 & -2.07894000 \\
\hline $\mathrm{N}$ & -4.16659600 & 1.27807100 & -1.20959400 \\
\hline $\mathrm{O}$ & -3.22874400 & -3.05413600 & -1.68579900 \\
\hline $\mathrm{O}$ & -2.90856600 & 3.10607600 & -1.88277400 \\
\hline $\mathrm{C}$ & -5.06431400 & -1.63811200 & 0.00000000 \\
\hline C & -4.66717300 & 1.87165700 & 0.00000000 \\
\hline $\mathrm{H}$ & 6.15527900 & -1.24027700 & 0.00000000 \\
\hline $\mathrm{H}$ & 5.02365400 & -2.63324800 & 0.00000000 \\
\hline $\mathrm{H}$ & 4.22524600 & 2.98006600 & 0.00000000 \\
\hline $\mathrm{H}$ & 5.68091300 & 1.92636000 & 0.00000000 \\
\hline $\mathrm{H}$ & 2.99254100 & 1.53391500 & -5.27653000 \\
\hline $\mathrm{H}$ & 2.30389200 & 2.83732500 & -4.25145200 \\
\hline $\mathrm{H}$ & 2.93393000 & -1.65992500 & -5.03885300 \\
\hline $\mathrm{H}$ & 2.30706600 & -2.81079900 & -3.81058700 \\
\hline
\end{tabular}




$\begin{array}{cccc}\mathrm{H} & -3.00094500 & 1.49121300 & -5.28864500 \\ \mathrm{H} & -2.35574400 & 2.80236500 & -4.24514700 \\ \mathrm{H} & -3.01302200 & -1.67536900 & -5.05373400 \\ \mathrm{H} & -2.35557700 & -2.84596500 & -3.86107000 \\ \mathrm{H} & -4.34370400 & 2.91639300 & 0.00000000 \\ \mathrm{H} & -5.76718000 & 1.81933500 & 0.00000000 \\ \mathrm{H} & -4.94269700 & -2.72417600 & 0.00000000 \\ \mathrm{H} & -6.13101900 & -1.37867600 & 0.00000000 \\ \mathrm{H} & -3.00094500 & 1.49121300 & 5.28864500 \\ \mathrm{H} & -2.35574400 & 2.80236500 & 4.24514700 \\ \mathrm{H} & -2.35557700 & -2.84596500 & 3.86107000 \\ \mathrm{H} & -3.01302200 & -1.67536900 & 5.05373400 \\ \mathrm{H} & 4.59878100 & 0.03248200 & 4.06445200 \\ \mathrm{H} & 5.78811800 & 0.36251600 & 1.96296000 \\ \mathrm{H} & 4.59878100 & 0.03248200 & -4.06445200 \\ \mathrm{H} & 5.78811800 & 0.36251600 & -1.96296000 \\ \mathrm{H} & 1.20644300 & -0.20252300 & -6.03269900 \\ \mathrm{H} & -1.23124200 & -0.12864700 & -6.05213800 \\ \mathrm{H} & -5.82855100 & 0.24613700 & -1.95220300 \\ \mathrm{H} & 2.93393000 & -1.65992500 & 5.03885300 \\ \mathrm{H} & 2.30706600 & -2.81079900 & 3.81058700 \\ \mathrm{H} & 2.30389200 & 2.83732500 & 4.25145200 \\ \mathrm{H} & 2.99254100 & 1.53391500 & 5.27653000 \\ \mathrm{H} & -4.64606400 & -0.06782100 & -4.05944700 \\ \mathrm{~S} & 0.54358300 & -1.20291800 & 0.00000000 \\ \mathrm{H} & 0.52673700 & -2.12815100 & 0.97643300 \\ \mathrm{H} & 0.52673700 & -2.12815100 & -0.97643300\end{array}$

\section{$\mathrm{CO}_{2} @ \mathrm{CB}[6]$}

$\begin{array}{lrrr}\mathrm{C} & 1.77142900 & 4.79073300 & 0.05792700 \\ \mathrm{C} & 3.13278200 & 4.02955700 & -0.03192100 \\ \mathrm{~N} & 2.98362700 & 3.24159400 & -1.23043900 \\ \mathrm{C} & 1.86143600 & 3.59201600 & -1.96441700 \\ \mathrm{~N} & 1.14079000 & 4.49431300 & -1.19724100 \\ \mathrm{~N} & 1.16627000 & 4.23079500 & 1.24032100 \\ \mathrm{H} & 1.88829000 & 5.87868300 & 0.17236000 \\ \mathrm{~N} & 3.19773100 & 3.31672700 & 1.21273000 \\ \mathrm{H} & 4.00042500 & 4.69964400 & -0.12504400 \\ \mathrm{C} & 4.04228400 & 2.47330000 & -1.82802100 \\ \mathrm{O} & 1.59157600 & 3.22867200 & -3.08406200 \\ \mathrm{C} & 4.33207700 & 2.57014900 & 1.68826300 \\ \mathrm{C} & 2.04273500 & 3.44096600 & 1.96839500 \\ \mathrm{O} & 1.85850200 & 2.99652400 & 3.07602900 \\ \mathrm{~N} & 4.46182200 & 1.23473400 & 1.17223100 \\ \mathrm{C} & 5.02906500 & 0.89071200 & -0.10169100 \\ \mathrm{C} & 4.12721000 & 0.12709700 & 1.93553500 \\ \mathrm{C} & 5.08332600 & -0.67068400 & -0.06363700 \\ \mathrm{~N} & 4.22545100 & 1.16458900 & -1.26444600 \\ \mathrm{H} & 6.01932200 & 1.35810000 & -0.21047800 \\ \mathrm{~N} & 4.41280100 & -0.99268200 & 1.17023200 \\ \mathrm{O} & 3.72918300 & 0.13656900 & 3.07552100 \\ \mathrm{~N} & 4.42925400 & -1.05287000 & -1.28266000 \\ \mathrm{H} & 6.10819100 & -1.07049100 & -0.04785300 \\ \mathrm{C} & 3.92266400 & 0.02383000 & -1.99143400 \\ \mathrm{O} & 3.38299100 & -0.01742400 & -3.07093600 \\ \mathrm{C} & 4.35048200 & -2.31114900 & 1.74138000\end{array}$




\begin{tabular}{|c|c|c|c|}
\hline $\mathrm{C}$ & 4.28753600 & -2.40047200 & -1.75858100 \\
\hline $\mathrm{N}$ & 3.19814300 & -3.14550300 & -1.18667100 \\
\hline $\mathrm{N}$ & 3.26975500 & -3.13370000 & 1.27332800 \\
\hline C & 2.07916000 & -3.45652000 & -1.94083500 \\
\hline C & 3.26518300 & -3.88466600 & 0.04869700 \\
\hline C & 1.89029300 & -4.62775700 & 0.09235200 \\
\hline $\mathrm{N}$ & 1.29579900 & -4.30463400 & -1.17396800 \\
\hline C & 2.09178000 & -3.24936100 & 1.98992100 \\
\hline $\mathrm{N}$ & 1.25134900 & -4.07680100 & 1.25978700 \\
\hline C & 0.08692700 & -4.88503000 & -1.69189900 \\
\hline $\mathrm{C}$ & 0.04866300 & -4.60703300 & 1.84362400 \\
\hline $\mathrm{O}$ & 1.86032800 & -2.77243300 & 3.07516300 \\
\hline $\mathrm{O}$ & 1.85512400 & -3.10893900 & -3.07564200 \\
\hline $\mathrm{N}$ & -1.13480700 & -4.31899300 & -1.18689100 \\
\hline $\mathrm{N}$ & -1.16826900 & -4.12645100 & 1.24877600 \\
\hline C & -1.92172200 & -3.48244800 & -1.96342800 \\
\hline $\mathrm{C}$ & -1.75673500 & -4.67889600 & 0.05636700 \\
\hline C & -3.15195600 & -3.97622600 & -0.01448600 \\
\hline $\mathrm{N}$ & -3.07606300 & -3.22996300 & -1.24399000 \\
\hline $\mathrm{O}$ & -1.67048400 & -3.10013200 & -3.08143500 \\
\hline $\mathrm{C}$ & -4.14766000 & -2.45684500 & -1.80720100 \\
\hline $\mathrm{N}$ & -3.20801100 & -3.23165200 & 1.21361200 \\
\hline C & -2.05277100 & -3.33768700 & 1.96882600 \\
\hline $\mathrm{O}$ & -1.87025800 & -2.88249900 & 3.07247100 \\
\hline C & -4.34685400 & -2.49435500 & 1.69095700 \\
\hline $\mathrm{N}$ & -4.29558600 & -1.13374300 & -1.26638200 \\
\hline $\mathrm{N}$ & -4.48138400 & -1.15842000 & 1.17891000 \\
\hline C & -3.91798100 & -0.01507800 & -1.99038500 \\
\hline C & -5.05948500 & -0.81290900 & -0.09041000 \\
\hline $\mathrm{C}$ & -5.05413100 & 0.75054000 & -0.07370200 \\
\hline $\mathrm{N}$ & -4.34943100 & 1.09178200 & -1.27768200 \\
\hline $\mathrm{O}$ & -3.37793600 & -0.00588600 & -3.07064700 \\
\hline C & -4.18616800 & 2.42442500 & -1.78503400 \\
\hline $\mathrm{N}$ & -4.40293600 & 1.06782900 & 1.17061100 \\
\hline C & -4.13261600 & -0.05445800 & 1.93917800 \\
\hline $\mathrm{O}$ & -3.73567000 & -0.06412700 & 3.07952100 \\
\hline C & -4.33303400 & 2.38750000 & 1.73835100 \\
\hline $\mathrm{N}$ & -3.11373600 & 3.17688000 & -1.18687500 \\
\hline $\mathrm{N}$ & -3.25156700 & 3.20660800 & 1.26361100 \\
\hline $\mathrm{C}$ & -3.24481600 & 3.94072200 & 0.03017500 \\
\hline C & -2.07918900 & 3.34994200 & 1.98733100 \\
\hline C & -1.90271000 & 4.73814100 & 0.09360400 \\
\hline $\mathrm{N}$ & -1.25135000 & 4.18685900 & 1.25495100 \\
\hline $\mathrm{C}$ & -2.02239000 & 3.57124800 & -1.94681400 \\
\hline $\mathrm{N}$ & -1.29649000 & 4.46913100 & -1.17987000 \\
\hline $\mathrm{O}$ & -1.84731600 & 2.88414500 & 3.07718600 \\
\hline $\mathrm{O}$ & -1.78158300 & 3.24325200 & -3.08374500 \\
\hline C & -0.04808900 & 4.72092800 & 1.83240000 \\
\hline C & -0.08744000 & 5.07336000 & -1.67265200 \\
\hline $\mathrm{H}$ & 0.07272700 & -5.70564700 & 1.78079600 \\
\hline $\mathrm{H}$ & 0.03541500 & -4.29281000 & 2.89099500 \\
\hline $\mathrm{H}$ & 0.09356500 & -4.71307300 & -2.77145700 \\
\hline $\mathrm{H}$ & 0.09156400 & -5.96366700 & -1.48197000 \\
\hline $\mathrm{H}$ & -5.09339000 & -3.00154000 & -1.67409600 \\
\hline $\mathrm{H}$ & -3.92787900 & -2.34427100 & -2.87219200 \\
\hline $\mathrm{H}$ & -5.25741900 & -3.06081700 & 1.45407300 \\
\hline $\mathrm{H}$ & -4.23385800 & -2.40254100 & 2.77410000 \\
\hline $\mathrm{H}$ & -5.13135900 & 2.97063600 & -1.65161700 \\
\hline $\mathrm{H}$ & -3.95378400 & 2.33471800 & -2.84948200 \\
\hline $\mathrm{H}$ & -5.27965700 & 2.91272100 & 1.54961200 \\
\hline $\mathrm{H}$ & -4.18482700 & 2.25867900 & 2.81356700 \\
\hline
\end{tabular}




$\begin{array}{lrrr}\mathrm{H} & -0.09568300 & 4.94710100 & -2.75824000 \\ \mathrm{H} & -0.09561300 & 6.14111500 & -1.41765500 \\ \mathrm{H} & -0.03434100 & 4.41805100 & 2.88313600 \\ \mathrm{H} & -0.06995700 & 5.81892300 & 1.75823700 \\ \mathrm{H} & 4.98669900 & 3.03252700 & -1.74637900 \\ \mathrm{H} & 3.77977000 & 2.33659500 & -2.88058500 \\ \mathrm{H} & 4.21688000 & 2.47594100 & 2.77095400 \\ \mathrm{H} & 5.24568700 & 3.13277600 & 1.45419000 \\ \mathrm{H} & 4.12718900 & -4.56784400 & 0.02861000 \\ \mathrm{H} & 1.98989000 & -5.71862600 & 0.19633100 \\ \mathrm{H} & -3.99278200 & -4.68418100 & -0.05764500 \\ \mathrm{H} & -1.82607700 & -5.77383100 & 0.14117400 \\ \mathrm{H} & -6.06851300 & -1.24403500 & -0.17187900 \\ \mathrm{H} & -6.06277700 & 1.18973200 & -0.09200500 \\ \mathrm{H} & -2.04622900 & 5.82149500 & 0.22052700 \\ \mathrm{H} & 5.29801600 & -2.83355700 & 1.54978600 \\ \mathrm{H} & 4.20684400 & -2.18034000 & 2.81698000 \\ \mathrm{H} & 4.09605500 & -2.33924700 & -2.83294300 \\ \mathrm{H} & 5.22847800 & -2.93731600 & -1.57469900 \\ \mathrm{H} & -4.13290100 & 4.58793900 & -0.02512400 \\ \mathrm{C} & 0.00135100 & -0.98153200 & 0.01352000 \\ \mathrm{O} & -1.16352900 & -0.98807700 & 0.02861300 \\ \mathrm{O} & 1.16608400 & -0.97325700 & -0.00422400\end{array}$

\section{CO@CB[6]}

$\begin{array}{lrrr}\mathrm{C} & -5.10845300 & 0.69153700 & 0.07148800 \\ \mathrm{C} & -5.06936100 & -0.87142400 & 0.09678000 \\ \mathrm{~N} & -4.27945100 & -1.16292700 & 1.26492200 \\ \mathrm{C} & -3.96672900 & -0.03129900 & 2.00113500 \\ \mathrm{~N} & -4.44736900 & 1.05766100 & 1.29245900 \\ \mathrm{~N} & -4.43489100 & 1.01949300 & -1.16018600 \\ \mathrm{H} & -6.12945200 & 1.10100300 & 0.06027800 \\ \mathrm{~N} & -4.49531100 & -1.20894900 & -1.17673700 \\ \mathrm{H} & -6.06485200 & -1.32996500 & 0.19324200 \\ \mathrm{C} & -4.09952000 & -2.47897600 & 1.81512700 \\ \mathrm{O} & -3.43335400 & -0.00311600 & 3.08427400 \\ \mathrm{C} & -4.35223500 & -2.54235000 & -1.69749100 \\ \mathrm{C} & -4.16633800 & -0.09821300 & -1.93701500 \\ \mathrm{O} & -3.77899900 & -0.10132500 & -3.08061700 \\ \mathrm{~N} & -3.21248600 & -3.27836200 & -1.22021300 \\ \mathrm{C} & -3.15290300 & -4.00720100 & 0.01511800 \\ \mathrm{C} & -2.05093400 & -3.38649400 & -1.96963000 \\ \mathrm{C} & -1.78117400 & -4.74956900 & -0.06981400 \\ \mathrm{~N} & -3.02831200 & -3.23381300 & 1.22510500 \\ \mathrm{H} & -4.01273500 & -4.68975500 & 0.08879800 \\ \mathrm{~N} & -1.17070200 & -4.17253500 & -1.24157100 \\ \mathrm{O} & -1.86659800 & -2.93343000 & -3.07363000 \\ \mathrm{~N} & -1.16721300 & -4.45787200 & 1.19427300 \\ \mathrm{H} & -1.88376100 & -5.83784400 & -0.19559000 \\ \mathrm{C} & -1.90508000 & -3.57259700 & 1.96379100 \\ \mathrm{O} & -1.64993200 & -3.21520200 & 3.08871400 \\ \mathrm{C} & 0.03670500 & -4.67143700 & -1.84372900 \\ \mathrm{C} & 0.07143700 & -5.01395500 & 1.66766500 \\ \mathrm{~N} & 1.26802900 & -4.39419800 & 1.16504200 \\ \mathrm{~N} & 1.25147700 & -4.15872600 & -1.27493900 \\ \mathrm{C} & 2.00649100 & -3.51553200 & 1.94021200\end{array}$




\begin{tabular}{|c|c|c|c|}
\hline C & 1.88735000 & -4.68882800 & -0.09715100 \\
\hline C & 3.23439900 & -3.89710100 & -0.03391700 \\
\hline $\mathrm{N}$ & 3.12127400 & -3.15761700 & 1.19797500 \\
\hline $\mathrm{C}$ & 2.06255000 & -3.29001700 & -1.98402000 \\
\hline $\mathrm{N}$ & 3.23059200 & -3.13953900 & -1.25364500 \\
\hline C & 4.18211300 & -2.38595300 & 1.78619400 \\
\hline $\mathrm{C}$ & 4.31605400 & -2.32959800 & -1.73177100 \\
\hline $\mathrm{O}$ & 1.82818100 & -2.80642400 & -3.06583100 \\
\hline $\mathrm{O}$ & 1.76079100 & -3.17834000 & 3.07353900 \\
\hline $\mathrm{N}$ & 4.31350300 & -1.04744600 & 1.27889500 \\
\hline $\mathrm{N}$ & 4.39214100 & -1.00785600 & -1.16781300 \\
\hline C & 3.88925300 & 0.05069200 & 2.00974400 \\
\hline C & 5.02668400 & -0.68882000 & 0.08424800 \\
\hline C & 5.01135900 & 0.87494100 & 0.10823300 \\
\hline $\mathrm{N}$ & 4.25306300 & 1.17790100 & 1.29290800 \\
\hline $\mathrm{O}$ & 3.35585800 & 0.02801500 & 3.09308800 \\
\hline C & 4.05485200 & 2.49751800 & 1.82583300 \\
\hline $\mathrm{N}$ & 4.41921600 & 1.21934300 & -1.15624500 \\
\hline C & 4.11386700 & 0.11411800 & -1.93330900 \\
\hline $\mathrm{O}$ & 3.73411700 & 0.12406300 & -3.07945900 \\
\hline C & 4.29805400 & 2.55414300 & -1.67868400 \\
\hline $\mathrm{N}$ & 2.97217100 & 3.23352900 & 1.23189000 \\
\hline $\mathrm{N}$ & 3.16174400 & 3.30141100 & -1.21438600 \\
\hline C & 1.83650400 & 3.54304300 & 1.96418400 \\
\hline $\mathrm{C}$ & 3.08948600 & 4.01702700 & 0.02771700 \\
\hline C & 1.70892500 & 4.74356000 & -0.05562400 \\
\hline $\mathrm{N}$ & 1.08475000 & 4.41774000 & 1.19543800 \\
\hline $\mathrm{O}$ & 1.58115300 & 3.17388100 & 3.08524300 \\
\hline C & -0.14172500 & 4.98745700 & 1.68469500 \\
\hline $\mathrm{N}$ & 1.11633300 & 4.18247400 & -1.24270800 \\
\hline C & 2.00137800 & 3.39877700 & -1.96614600 \\
\hline $\mathrm{O}$ & 1.82225100 & 2.94372500 & -3.07014900 \\
\hline C & -0.10116300 & 4.66086300 & -1.83635500 \\
\hline $\mathrm{N}$ & -1.35165100 & 4.39380900 & 1.18149600 \\
\hline $\mathrm{N}$ & -1.30312500 & 4.12640000 & -1.25514800 \\
\hline $\mathrm{C}$ & -1.95037600 & 4.67905800 & -0.09201100 \\
\hline C & -2.13932000 & 3.30237100 & -1.99197500 \\
\hline $\mathrm{C}$ & -3.30406400 & 3.90103900 & -0.03586800 \\
\hline $\mathrm{N}$ & -3.31694000 & 3.17338800 & -1.27271200 \\
\hline $\mathrm{C}$ & -2.09479900 & 3.50758600 & 1.94430800 \\
\hline $\mathrm{N}$ & -3.18959400 & 3.13027000 & 1.17854800 \\
\hline $\mathrm{O}$ & -1.91183700 & 2.83607400 & -3.08243200 \\
\hline $\mathrm{O}$ & -1.86596800 & 3.17591400 & 3.08252300 \\
\hline C & -4.39105500 & 2.33923200 & -1.73473300 \\
\hline $\mathrm{C}$ & -4.27948800 & 2.40047200 & 1.77355000 \\
\hline $\mathrm{H}$ & 5.26066500 & -2.85787000 & -1.54171100 \\
\hline $\mathrm{H}$ & 4.16922300 & -2.20108300 & -2.80724300 \\
\hline $\mathrm{H}$ & 3.95785200 & -2.30174300 & 2.85284700 \\
\hline $\mathrm{H}$ & 5.13664000 & -2.91263700 & 1.64319700 \\
\hline $\mathrm{H}$ & 4.98756500 & 3.06911800 & 1.71199600 \\
\hline $\mathrm{H}$ & 3.81552900 & 2.38056300 & 2.88619000 \\
\hline $\mathrm{H}$ & 5.21082700 & 3.11612600 & -1.43944500 \\
\hline $\mathrm{H}$ & 4.19150300 & 2.45569900 & -2.76194700 \\
\hline $\mathrm{H}$ & -0.15052300 & 6.06046900 & 1.45071200 \\
\hline $\mathrm{H}$ & -0.14865700 & 4.83990900 & 2.76771800 \\
\hline $\mathrm{H}$ & -0.12837600 & 5.75951200 & -1.77056500 \\
\hline $\mathrm{H}$ & -0.08705400 & 4.35002400 & -2.88473700 \\
\hline $\mathrm{H}$ & -4.06713200 & 2.33001500 & 2.84351800 \\
\hline $\mathrm{H}$ & -5.21709800 & 2.95103600 & 1.61204200 \\
\hline $\mathrm{H}$ & -4.25070000 & 2.20864600 & -2.81081900 \\
\hline $\mathrm{H}$ & -5.34341300 & 2.85117500 & -1.53932800 \\
\hline
\end{tabular}




$\begin{array}{lrrr}\mathrm{H} & -5.04051100 & -3.03970100 & 1.71126500 \\ \mathrm{H} & -3.85442500 & -2.35395200 & 2.87332200 \\ \mathrm{H} & -4.23523200 & -2.44220200 & -2.77946900 \\ \mathrm{H} & -5.26139400 & -3.11390800 & -1.46806400 \\ \mathrm{H} & 2.02647100 & -5.77520800 & -0.20192200 \\ \mathrm{H} & 4.12051700 & -4.54856000 & -0.00118900 \\ \mathrm{H} & 6.01513800 & 1.31898500 & 0.18418600 \\ \mathrm{H} & 6.04092000 & -1.11447400 & 0.11087500 \\ \mathrm{H} & 3.94034200 & 4.70972400 & 0.10999900 \\ \mathrm{H} & 1.79828400 & 5.83553000 & -0.15811700 \\ \mathrm{H} & -4.18234500 & 4.56143300 & 0.01930400 \\ \mathrm{H} & 0.04556000 & -5.77001500 & -1.77641600 \\ \mathrm{H} & 0.01957700 & -4.36207000 & -2.89243800 \\ \mathrm{H} & 0.08057500 & -4.88020700 & 2.75243500 \\ \mathrm{H} & 0.09561000 & -6.08354800 & 1.41983600 \\ \mathrm{H} & -2.07847500 & 5.76517900 & -0.21269100 \\ \mathrm{C} & 1.13874600 & -0.69140100 & -0.09702300 \\ \mathrm{O} & 1.35479400 & 0.42360900 & -0.11165500\end{array}$

$\mathrm{N}_{2} @ \mathrm{CB}[6]$

$\begin{array}{lrrr}\mathrm{C} & 5.12642600 & 0.62806100 & 0.07506500 \\ \mathrm{C} & 4.68035300 & 2.12550900 & 0.11125000 \\ \mathrm{~N} & 3.82863000 & 2.18696000 & 1.27126000 \\ \mathrm{C} & 3.83682600 & 1.01273900 & 2.00761100 \\ \mathrm{~N} & 4.60264400 & 0.09884300 & 1.30211500 \\ \mathrm{~N} & 4.54448400 & 0.13882900 & -1.15123200 \\ \mathrm{H} & 6.21883400 & 0.50086500 & 0.04736600 \\ \mathrm{~N} & 4.05239700 & 2.31419100 & -1.16712200 \\ \mathrm{H} & 5.51978500 & 2.82788500 & 0.22320800 \\ \mathrm{C} & 3.30839900 & 3.40547100 & 1.82850200 \\ \mathrm{O} & 3.32768400 & 0.83813700 & 3.08853600 \\ \mathrm{C} & 3.57531400 & 3.56986500 & -1.68195000 \\ \mathrm{C} & 4.01346100 & 1.15794500 & -1.92995200 \\ \mathrm{O} & 3.64465100 & 1.06814200 & -3.07603700 \\ \mathrm{~N} & 2.28102700 & 3.98646700 & -1.21210200 \\ \mathrm{C} & 2.02201000 & 4.65957000 & 0.02903100 \\ \mathrm{C} & 1.13594100 & 3.78439900 & -1.96675700 \\ \mathrm{C} & 0.50662100 & 5.02497500 & -0.06814300 \\ \mathrm{~N} & 2.08477900 & 3.86371600 & 1.22963000 \\ \mathrm{H} & 2.67756900 & 5.53822100 & 0.12223600 \\ \mathrm{~N} & 0.07553000 & 4.30671200 & -1.24242000 \\ \mathrm{O} & 1.08312300 & 3.30077300 & -3.07204700 \\ \mathrm{~N} & -0.02269300 & 4.58549300 & 1.19135200 \\ \mathrm{H} & 0.32569100 & 6.10256200 & -0.19691200 \\ \mathrm{C} & 0.90777600 & 3.90579600 & 1.96158400 \\ \mathrm{O} & 0.74229200 & 3.48650200 & 3.08182500 \\ \mathrm{C} & -1.21763000 & 4.46759900 & -1.85067600 \\ \mathrm{C} & -1.36042700 & 4.82471700 & 1.66347100 \\ \mathrm{~N} & -2.37057200 & 3.93176000 & 1.16327800 \\ \mathrm{~N} & -2.25759900 & 3.66435400 & -1.26988600 \\ \mathrm{C} & -2.87206600 & 2.89719300 & 1.93713200 \\ \mathrm{C} & -3.02241000 & 4.04825900 & -0.11096000 \\ \mathrm{C} & -4.14065000 & 2.95934800 & -0.04493700 \\ \mathrm{~N} & -3.84484000 & 2.25991700 & 1.18091300 \\ \mathrm{C} & -2.85344600 & 2.64173200 & -1.99031100 \\ \mathrm{~N} & -3.96652800 & 2.23545100 & -1.27176700\end{array}$




\begin{tabular}{|c|c|c|c|}
\hline C & -4.70395000 & 1.27012900 & 1.77448500 \\
\hline C & -4.79964700 & 1.15869100 & -1.72807000 \\
\hline $\mathrm{O}$ & -2.50873600 & 2.22720300 & -3.07095100 \\
\hline $\mathrm{O}$ & -2.56728700 & 2.64171800 & 3.07715300 \\
\hline $\mathrm{N}$ & -4.50764600 & -0.07129600 & 1.29910700 \\
\hline $\mathrm{N}$ & -4.50964700 & -0.12961600 & -1.15410900 \\
\hline $\mathrm{C}$ & -3.75891300 & -0.99109800 & 2.01386800 \\
\hline C & -5.06066500 & -0.60965300 & 0.08839800 \\
\hline C & -4.61325100 & -2.10699400 & 0.12395100 \\
\hline $\mathrm{N}$ & -3.76270800 & -2.16949200 & 1.28425000 \\
\hline $\mathrm{O}$ & -3.24919800 & -0.81650900 & 3.09455200 \\
\hline C & -3.24339300 & -3.38801200 & 1.84285100 \\
\hline $\mathrm{N}$ & -3.98445200 & -2.29506800 & -1.15436800 \\
\hline C & -3.97028300 & -1.14613100 & -1.92835200 \\
\hline $\mathrm{O}$ & -3.61014000 & -1.06012600 & -3.07758000 \\
\hline C & -3.50790800 & -3.55146100 & -1.66773500 \\
\hline $\mathrm{N}$ & -2.02143500 & -3.85051100 & 1.24558300 \\
\hline $\mathrm{N}$ & -2.21409900 & -3.96559200 & -1.19750300 \\
\hline C & -0.83886500 & -3.87252400 & 1.96831800 \\
\hline C & -1.95611800 & -4.64123500 & 0.04235900 \\
\hline C & -0.44022200 & -5.00610100 & -0.05432700 \\
\hline $\mathrm{N}$ & 0.09373600 & -4.54749300 & 1.19665700 \\
\hline $\mathrm{O}$ & -0.67049800 & -3.44390800 & 3.08469600 \\
\hline C & 1.42762700 & -4.79703300 & 1.67334300 \\
\hline $\mathrm{N}$ & -0.01294300 & -4.30539900 & -1.23909400 \\
\hline C & -1.07085900 & -3.77008500 & -1.95620200 \\
\hline $\mathrm{O}$ & -1.01906200 & -3.28471100 & -3.06079900 \\
\hline C & 1.28171900 & -4.46171000 & -1.84224900 \\
\hline $\mathrm{N}$ & 2.44746300 & -3.91556200 & 1.17148700 \\
\hline $\mathrm{N}$ & 2.31625400 & -3.64841600 & -1.26254300 \\
\hline C & 3.08951300 & -4.03102800 & -0.10761900 \\
\hline $\mathrm{C}$ & 2.92068000 & -2.64123100 & -1.99860200 \\
\hline C & 4.20472000 & -2.93956600 & -0.04867000 \\
\hline $\mathrm{N}$ & 4.03878100 & -2.23429800 & -1.28711800 \\
\hline C & 2.94266400 & -2.87073000 & 1.93520300 \\
\hline $\mathrm{N}$ & 3.89710300 & -2.21992800 & 1.16427800 \\
\hline $\mathrm{O}$ & 2.57736800 & -2.23792900 & -3.08374500 \\
\hline $\mathrm{O}$ & 2.64574100 & -2.61411300 & 3.07691100 \\
\hline C & 4.85443100 & -1.14097600 & -1.73647400 \\
\hline C & 4.77688600 & -1.24931500 & 1.76458200 \\
\hline $\mathrm{H}$ & -5.84872500 & 1.41600700 & -1.52702400 \\
\hline $\mathrm{H}$ & -4.63743000 & 1.06417100 & -2.80486000 \\
\hline $\mathrm{H}$ & -4.48560800 & 1.26421800 & 2.84565200 \\
\hline $\mathrm{H}$ & -5.75314700 & 1.55107700 & 1.60606000 \\
\hline $\mathrm{H}$ & -4.00866200 & -4.17431300 & 1.75854100 \\
\hline $\mathrm{H}$ & -3.02628200 & -3.19119600 & 2.89622900 \\
\hline $\mathrm{H}$ & -4.23749400 & -4.33549300 & -1.42552700 \\
\hline $\mathrm{H}$ & -3.42882100 & -3.43373500 & -2.75148500 \\
\hline $\mathrm{H}$ & 1.70068400 & -5.83191500 & 1.42776500 \\
\hline $\mathrm{H}$ & 1.40486400 & -4.66343000 & 2.75781300 \\
\hline $\mathrm{H}$ & 1.58103800 & -5.51973400 & -1.78511100 \\
\hline $\mathrm{H}$ & 1.18359700 & -4.15790100 & -2.88809800 \\
\hline $\mathrm{H}$ & 4.56223200 & -1.24823800 & 2.83648700 \\
\hline $\mathrm{H}$ & 5.82034200 & -1.54736700 & 1.59041200 \\
\hline $\mathrm{H}$ & 4.68909300 & -1.04297100 & -2.81246000 \\
\hline $\mathrm{H}$ & 5.90761700 & -1.38352100 & -1.53854200 \\
\hline $\mathrm{H}$ & 4.07216400 & 4.19331300 & 1.74271900 \\
\hline $\mathrm{H}$ & 3.09163800 & 3.21046500 & 2.88232100 \\
\hline $\mathrm{H}$ & 3.49528200 & 3.45129700 & -2.76550000 \\
\hline $\mathrm{H}$ & 4.30520500 & 4.35368700 & -1.44098700 \\
\hline $\mathrm{H}$ & -3.41661400 & 5.06751500 & -0.23939000 \\
\hline
\end{tabular}




$$
\begin{array}{rrr}
-5.15538500 & 3.38197100 & 0.00024000 \\
-5.45226000 & -2.81008900 & 0.23508300 \\
-6.15350700 & -0.48255400 & 0.08699500 \\
-2.61150300 & -5.52043500 & 0.13215300 \\
-0.25830500 & -6.08532500 & -0.16752900 \\
5.21955200 & -3.35992100 & 0.01181500 \\
-1.51072000 & 5.52770700 & -1.80025000 \\
-1.11783200 & 4.15751900 & -2.89453700 \\
-1.33785500 & 4.69623500 & 2.74858300 \\
-1.64162400 & 5.85620500 & 1.41275200 \\
3.48562100 & -5.04914900 & -0.23879700 \\
-1.03720100 & -0.85505100 & -0.18110500 \\
-1.38339700 & 0.18820900 & -0.10405600
\end{array}
$$

$\mathrm{NO}_{2} @ \mathrm{CB}[6]$

$\begin{array}{lrrr}\mathrm{C} & 4.28622700 & 2.81067100 & 0.03821000 \\ \mathrm{C} & 3.24250100 & 3.97098000 & 0.09831500 \\ \mathrm{~N} & 2.45408100 & 3.64163000 & 1.25899100 \\ \mathrm{C} & 2.99632800 & 2.60007900 & 1.99572600 \\ \mathrm{~N} & 4.07959900 & 2.11816300 & 1.27804800 \\ \mathrm{~N} & 3.93026200 & 2.11074800 & -1.17289300 \\ \mathrm{H} & 5.32587700 & 3.16514300 & -0.02522700 \\ \mathrm{~N} & 2.58711700 & 3.89152100 & -1.17687800 \\ \mathrm{H} & 3.70197200 & 4.96292100 & 0.22324000 \\ \mathrm{C} & 1.46354400 & 4.51178000 & 1.83199700 \\ \mathrm{O} & 2.63468500 & 2.22541500 & 3.08520700 \\ \mathrm{C} & 1.61221400 & 4.82589400 & -1.67297200 \\ \mathrm{C} & 3.01307400 & 2.81575000 & -1.93984600 \\ \mathrm{O} & 2.69340800 & 2.57466300 & -3.07892800 \\ \mathrm{~N} & 0.26673900 & 4.63745700 & -1.19950500 \\ \mathrm{C} & -0.25115700 & 5.11257000 & 0.05233500 \\ \mathrm{C} & -0.68576900 & 3.98332500 & -1.96550600 \\ \mathrm{C} & -1.77672900 & 4.78699800 & -0.03979600 \\ \mathrm{~N} & 0.15908400 & 4.40374300 & 1.23874900 \\ \mathrm{H} & -0.04170200 & 6.18694200 & 0.16264500 \\ \mathrm{~N} & -1.86394300 & 3.98584300 & -1.23551300 \\ \mathrm{O} & -0.53232300 & 3.54967000 & -3.08208100 \\ \mathrm{~N} & -2.05154600 & 4.12948700 & 1.20661900 \\ \mathrm{H} & -2.40775700 & 5.68291600 & -0.13761500 \\ \mathrm{C} & -0.91281800 & 3.90956800 & 1.96532800 \\ \mathrm{O} & -0.87153300 & 3.43491200 & 3.07499000 \\ \mathrm{C} & -3.09966200 & 3.55975700 & -1.83400000 \\ \mathrm{C} & -3.35547900 & 3.75120800 & 1.68221500 \\ \mathrm{~N} & -3.87190400 & 2.51103700 & 1.16956700 \\ \mathrm{~N} & -3.66095300 & 2.36485200 & -1.26710200 \\ \mathrm{C} & -3.88405800 & 1.35712300 & 1.93728200 \\ \mathrm{C} & -4.51197300 & 2.34640700 & -0.10570600 \\ \mathrm{C} & -5.02712900 & 0.87215100 & -0.06318600 \\ \mathrm{~N} & -4.47783000 & 0.36631200 & 1.16940100 \\ \mathrm{C} & -3.71725300 & 1.18638500 & -1.99489300 \\ \mathrm{~N} & -4.52013600 & 0.31074800 & -1.28247100 \\ \mathrm{C} & -4.82690000 & -0.90381800 & 1.74775200 \\ \mathrm{C} & -4.78805400 & -1.01864500 & -1.75378000 \\ \mathrm{O} & -3.21861100 & 0.98451800 & -3.07596500 \\ \mathrm{O} & -3.51035800 & 1.25280700 & 3.08062600 \\ \mathrm{~N} & -4.05314400 & -2.02244800 & 1.28500400\end{array}$




\begin{tabular}{|c|c|c|c|}
\hline $\mathrm{N}$ & -3.97400300 & -2.05382300 & -1.17347400 \\
\hline C & -2.96486200 & -2.48965200 & 2.00100100 \\
\hline C & -4.27471400 & -2.73781900 & 0.05928000 \\
\hline C & -3.19850300 & -3.87013000 & 0.10887400 \\
\hline $\mathrm{N}$ & -2.41593000 & -3.53336800 & 1.27092400 \\
\hline $\mathrm{O}$ & -2.59760100 & -2.10298600 & 3.08455900 \\
\hline C & -1.42365100 & -4.39779300 & 1.85084800 \\
\hline $\mathrm{N}$ & -2.54039800 & -3.75935600 & -1.16245200 \\
\hline $\mathrm{C}$ & -3.01614400 & -2.70807900 & -1.93047800 \\
\hline $\mathrm{O}$ & -2.69834900 & -2.45233400 & -3.06718200 \\
\hline C & -1.56159600 & -4.68117800 & -1.67211200 \\
\hline $\mathrm{N}$ & -0.12042400 & -4.29829900 & 1.25326600 \\
\hline $\mathrm{N}$ & -0.22184100 & -4.49876200 & -1.18210500 \\
\hline C & 0.95625100 & -3.79782400 & 1.96928100 \\
\hline $\mathrm{C}$ & 0.28015400 & -5.00550000 & 0.06394100 \\
\hline C & 1.81599700 & -4.72293000 & -0.01799000 \\
\hline $\mathrm{N}$ & 2.09051500 & -4.02854100 & 1.20989200 \\
\hline $\mathrm{O}$ & 0.91723300 & -3.30861200 & 3.07274600 \\
\hline C & 3.39738900 & -3.66652200 & 1.69105400 \\
\hline $\mathrm{N}$ & 1.94173400 & -3.97665400 & -1.24327900 \\
\hline $\mathrm{C}$ & 0.75998400 & -3.90311900 & -1.95888900 \\
\hline $\mathrm{O}$ & 0.61836100 & -3.46088100 & -3.07399700 \\
\hline C & 3.18450700 & -3.53779400 & -1.81419700 \\
\hline $\mathrm{N}$ & 3.92767800 & -2.43180100 & 1.18231300 \\
\hline $\mathrm{N}$ & 3.71397400 & -2.32313400 & -1.25751600 \\
\hline C & 4.56222200 & -2.26859300 & -0.09567900 \\
\hline C & 3.73188800 & -1.14857600 & -1.99356400 \\
\hline C & 5.04122600 & -0.78160500 & -0.07040600 \\
\hline $\mathrm{N}$ & 4.50890300 & -0.24339700 & -1.28970800 \\
\hline $\mathrm{C}$ & 3.91824600 & -1.27137200 & 1.93950300 \\
\hline $\mathrm{N}$ & 4.48987900 & -0.27468600 & 1.16133600 \\
\hline $\mathrm{O}$ & 3.22475100 & -0.97000100 & -3.07481600 \\
\hline $\mathrm{O}$ & 3.54536100 & -1.16467700 & 3.08283900 \\
\hline C & 4.74492200 & 1.08645600 & -1.77527000 \\
\hline C & 4.84601900 & 0.99477900 & 1.74035000 \\
\hline $\mathrm{H}$ & -5.84736100 & -1.24767400 & -1.57194600 \\
\hline $\mathrm{H}$ & -4.58386100 & -1.02337000 & -2.82760000 \\
\hline $\mathrm{H}$ & -4.65115000 & -0.81817400 & 2.82313900 \\
\hline $\mathrm{H}$ & -5.88923100 & -1.10922700 & 1.55597100 \\
\hline $\mathrm{H}$ & -1.77270200 & -5.43960800 & 1.78683600 \\
\hline $\mathrm{H}$ & -1.31546300 & -4.10376900 & 2.89849100 \\
\hline $\mathrm{H}$ & -1.88491000 & -5.70575700 & -1.44341500 \\
\hline $\mathrm{H}$ & -1.52428700 & -4.53220200 & -2.75434700 \\
\hline $\mathrm{H}$ & 4.09678200 & -4.47915500 & 1.45401300 \\
\hline $\mathrm{H}$ & 3.31400700 & -3.54651800 & 2.77422100 \\
\hline $\mathrm{H}$ & 3.93312300 & -4.33633300 & -1.70465300 \\
\hline $\mathrm{H}$ & 2.99380000 & -3.34877800 & -2.87395700 \\
\hline $\mathrm{H}$ & 4.66912400 & 0.91052800 & 2.81565200 \\
\hline $\mathrm{H}$ & 5.90976800 & 1.19364700 & 1.54901700 \\
\hline $\mathrm{H}$ & 4.51095200 & 1.08330800 & -2.84311200 \\
\hline $\mathrm{H}$ & 5.80609000 & 1.33115700 & -1.62377700 \\
\hline $\mathrm{H}$ & 1.81157100 & 5.55319700 & 1.75480800 \\
\hline $\mathrm{H}$ & 1.35847900 & 4.23028000 & 2.88340500 \\
\hline $\mathrm{H}$ & 1.58392500 & 4.70065900 & -2.75834300 \\
\hline $\mathrm{H}$ & 1.93534400 & 5.84393400 & -1.41938500 \\
\hline $\mathrm{H}$ & -5.31808500 & 3.08701500 & -0.21828500 \\
\hline $\mathrm{H}$ & -6.12456900 & 0.79474400 & -0.04328300 \\
\hline $\mathrm{H}$ & -3.63128500 & -4.87521600 & 0.22377800 \\
\hline $\mathrm{H}$ & -5.30581800 & -3.12023600 & 0.03223600 \\
\hline $\mathrm{H}$ & 0.04006500 & -6.07479200 & 0.16102300 \\
\hline $\mathrm{H}$ & 2.42399500 & -5.63822400 & -0.07120200 \\
\hline
\end{tabular}




$\begin{array}{lrrr}\mathrm{H} & 6.13660000 & -0.67745300 & -0.06094400 \\ \mathrm{H} & -3.83839100 & 4.37233100 & -1.75907100 \\ \mathrm{H} & -2.88547900 & 3.34752400 & -2.88502600 \\ \mathrm{H} & -3.27330100 & 3.63082400 & 2.76543600 \\ \mathrm{H} & -4.06330700 & 4.55635500 & 1.44443300 \\ \mathrm{H} & 5.38619800 & -2.99053800 & -0.19891700 \\ \mathrm{~N} & -0.46115400 & -1.29027900 & -0.10351800 \\ \mathrm{O} & -1.36256700 & -0.50906400 & -0.03204400 \\ \mathrm{O} & 0.72616700 & -1.19729800 & -0.00873100\end{array}$

\section{NO@CB[6]}

$\begin{array}{lrrr}\mathrm{C} & 4.62637500 & 2.22953100 & 0.13245600 \\ \mathrm{C} & 5.10957600 & 0.74437200 & 0.08361400 \\ \mathrm{~N} & 4.54292100 & 0.25311500 & -1.14925000 \\ \mathrm{C} & 3.99866800 & 1.26832800 & -1.92388800 \\ \mathrm{~N} & 4.00786300 & 2.41817100 & -1.15033200 \\ \mathrm{~N} & 3.76077400 & 2.25561400 & 1.28358400 \\ \mathrm{H} & 5.44689800 & 2.95105600 & 0.26193000 \\ \mathrm{~N} & 4.59579800 & 0.19097900 & 1.30381100 \\ \mathrm{H} & 6.20487200 & 0.64411300 & 0.05802300 \\ \mathrm{C} & 4.88052700 & -1.01584600 & -1.74222600 \\ \mathrm{O} & 3.64236700 & 1.18183100 & -3.07417900 \\ \mathrm{C} & 4.80984400 & -1.15434100 & 1.75785300 \\ \mathrm{C} & 3.79736300 & 1.07586400 & 2.01047500 \\ \mathrm{O} & 3.28501700 & 0.87728000 & 3.08566900 \\ \mathrm{~N} & 3.95669300 & -2.14600900 & 1.15359700 \\ \mathrm{C} & 4.27768000 & -2.84403800 & -0.06879200 \\ \mathrm{C} & 3.03108400 & -2.83579700 & 1.92549500 \\ \mathrm{C} & 3.18926400 & -3.96156900 & -0.13552800 \\ \mathrm{~N} & 4.08953800 & -2.12960300 & -1.29852900 \\ \mathrm{H} & 5.30277500 & -3.23995400 & -0.01705400 \\ \mathrm{~N} & 2.55819300 & -3.88393800 & 1.15182900 \\ \mathrm{O} & 2.73982300 & -2.60509600 & 3.07416300 \\ \mathrm{~N} & 2.39516100 & -3.57738600 & -1.27589200 \\ \mathrm{H} & 3.60872900 & -4.96729400 & -0.28803000 \\ \mathrm{C} & 2.97132800 & -2.54758900 & -2.00333300 \\ \mathrm{O} & 2.60741400 & -2.13463200 & -3.07812000 \\ \mathrm{C} & 1.56764600 & -4.79985300 & 1.65117800 \\ \mathrm{C} & 1.37045400 & -4.40173900 & -1.85667200 \\ \mathrm{~N} & 0.08066700 & -4.28013500 & -1.23397500 \\ \mathrm{~N} & 0.22135200 & -4.57892500 & 1.19516400 \\ \mathrm{C} & -1.00477100 & -3.79413000 & -1.94752400 \\ \mathrm{C} & -0.31548900 & -5.01951600 & -0.06045500 \\ \mathrm{C} & -1.83611800 & -4.68962700 & 0.06040000 \\ \mathrm{~N} & -2.13438900 & -4.03641300 & -1.18129100 \\ \mathrm{C} & -0.70768200 & -3.90361500 & 1.97152000 \\ \mathrm{~N} & -1.89341800 & -3.88185800 & 1.25368500 \\ \mathrm{C} & -3.44945900 & -3.67573900 & -1.64039800 \\ \mathrm{C} & -3.11516600 & -3.43376700 & 1.86125100 \\ \mathrm{O} & -0.53344700 & -3.47325300 & 3.08627200 \\ \mathrm{O} & -0.97982400 & -3.31079400 & -3.05377000 \\ \mathrm{~N} & -3.97144600 & -2.43066400 & -1.14500600 \\ \mathrm{~N} & -3.66520600 & -2.23731700 & 1.28344500 \\ \mathrm{C} & -3.93504800 & -1.27750300 & -1.91222700 \\ & -4.55680900 & -2.22633900 & 0.14951700 \\ \mathrm{C} & -4.03898100 & -0.74180100 & 0.09402500 \\ \mathrm{~N} & & -0.25514500 & -1.13624500\end{array}$




\begin{tabular}{|c|c|c|c|}
\hline $\mathrm{O}$ & -3.57470000 & -1.19299300 & -3.06140100 \\
\hline $\mathrm{C}$ & -4.79775800 & 1.01263800 & -1.73308600 \\
\hline $\mathrm{N}$ & -4.53145900 & -0.18503100 & 1.31532900 \\
\hline $\mathrm{C}$ & -3.71684700 & -1.05994600 & 2.01412300 \\
\hline $\mathrm{O}$ & -3.19742500 & -0.85551700 & 3.08511800 \\
\hline C & -4.74801100 & 1.16151000 & 1.76674000 \\
\hline $\mathrm{N}$ & -4.00810700 & 2.12579100 & -1.28773400 \\
\hline $\mathrm{N}$ & -3.89533200 & 2.15390300 & 1.16547400 \\
\hline C & -2.89520000 & 2.55007400 & -1.99540400 \\
\hline $\mathrm{C}$ & -4.20471600 & 2.84562700 & -0.06251400 \\
\hline $\mathrm{C}$ & -3.11480900 & 3.96245300 & -0.12603200 \\
\hline $\mathrm{N}$ & -2.33065100 & 3.59136700 & -1.27620600 \\
\hline $\mathrm{O}$ & -2.52712100 & 2.13384600 & -3.06771200 \\
\hline $\mathrm{C}$ & -1.30799600 & 4.41851800 & -1.85417300 \\
\hline $\mathrm{N}$ & -2.47118900 & 3.86996300 & 1.15440700 \\
\hline $\mathrm{C}$ & -2.95526700 & 2.82966900 & 1.93134700 \\
\hline $\mathrm{O}$ & -2.66198500 & 2.59553400 & 3.07886800 \\
\hline C & -1.48685600 & 4.79020700 & 1.65752500 \\
\hline $\mathrm{N}$ & -0.01560000 & 4.29352900 & -1.23591700 \\
\hline $\mathrm{N}$ & -0.14040400 & 4.58234000 & 1.19567800 \\
\hline C & 0.38756300 & 5.02872400 & -0.06154400 \\
\hline $\mathrm{C}$ & 0.79558900 & 3.90977000 & 1.96595400 \\
\hline C & 1.91002500 & 4.70070000 & 0.04918600 \\
\hline $\mathrm{N}$ & 1.97726300 & 3.88993000 & 1.24027900 \\
\hline $\mathrm{C}$ & 1.06581100 & 3.81147000 & -1.95762500 \\
\hline $\mathrm{N}$ & 2.20000700 & 4.05182800 & -1.19766200 \\
\hline $\mathrm{O}$ & 0.62946900 & 3.48013000 & 3.08209900 \\
\hline $\mathrm{O}$ & 1.03472400 & 3.32976800 & -3.06458500 \\
\hline $\mathrm{C}$ & 3.20510200 & 3.45473600 & 1.84826400 \\
\hline $\mathrm{C}$ & 3.50845900 & 3.66841300 & -1.65696700 \\
\hline $\mathrm{H}$ & -3.86637400 & -4.23653800 & 1.80502800 \\
\hline $\mathrm{H}$ & -2.88630800 & -3.21160600 & 2.90720900 \\
\hline $\mathrm{H}$ & -3.38767700 & -3.57346800 & -2.72671000 \\
\hline $\mathrm{H}$ & -4.14558200 & -4.48218800 & -1.37635300 \\
\hline $\mathrm{H}$ & -5.85696900 & 1.23588000 & -1.54350800 \\
\hline $\mathrm{H}$ & -4.62427400 & 0.90823300 & -2.80719200 \\
\hline $\mathrm{H}$ & -5.79923000 & 1.42462900 & 1.58444500 \\
\hline $\mathrm{H}$ & -4.53993500 & 1.17579300 & 2.83973200 \\
\hline $\mathrm{H}$ & -1.62979500 & 5.47075100 & -1.81232500 \\
\hline $\mathrm{H}$ & -1.19234300 & 4.10530600 & -2.89546700 \\
\hline $\mathrm{H}$ & -1.79140100 & 5.81320100 & 1.39994400 \\
\hline $\mathrm{H}$ & -1.46978800 & 4.66671200 & 2.74330500 \\
\hline $\mathrm{H}$ & 3.44313600 & 3.55973600 & -2.74250800 \\
\hline $\mathrm{H}$ & 4.21765000 & 4.46562300 & -1.39934400 \\
\hline $\mathrm{H}$ & 2.98150700 & 3.24290000 & 2.89745000 \\
\hline $\mathrm{H}$ & 3.95092100 & 4.26142600 & 1.78003800 \\
\hline $\mathrm{H}$ & 5.93871000 & -1.23778200 & -1.54684100 \\
\hline $\mathrm{H}$ & 4.71228800 & -0.91406500 & -2.81738400 \\
\hline $\mathrm{H}$ & 4.59786500 & -1.16717300 & 2.83016400 \\
\hline $\mathrm{H}$ & 5.86136300 & -1.42003300 & 1.57961300 \\
\hline $\mathrm{H}$ & -0.11195400 & -6.09156100 & -0.20116900 \\
\hline $\mathrm{H}$ & -2.46832300 & -5.58260400 & 0.17607900 \\
\hline $\mathrm{H}$ & -6.13405800 & -0.64112200 & 0.06243800 \\
\hline $\mathrm{H}$ & -5.37475000 & -2.94472200 & 0.30856700 \\
\hline $\mathrm{H}$ & -5.22997800 & 3.24236500 & -0.02072900 \\
\hline $\mathrm{H}$ & -3.53401800 & 4.97047900 & -0.26374800 \\
\hline $\mathrm{H}$ & 2.54134700 & 5.59451000 & 0.16232800 \\
\hline $\mathrm{H}$ & 1.86400300 & -5.82307900 & 1.38569000 \\
\hline $\mathrm{H}$ & 1.55558200 & -4.68355900 & 2.73778800 \\
\hline $\mathrm{H}$ & 1.25247700 & -4.08201600 & -2.89575800 \\
\hline $\mathrm{H}$ & 1.69237500 & -5.45435400 & -1.82230500 \\
\hline
\end{tabular}




$\begin{array}{lrrr}\mathrm{H} & 0.18202500 & 6.10100200 & -0.19712000 \\ \mathrm{~N} & -1.21997700 & -0.67739000 & -0.31830300 \\ \mathrm{O} & -1.30447500 & 0.44930700 & -0.08879700\end{array}$

$\mathrm{Br}_{2} @ \mathrm{CB}[6]$

\begin{tabular}{|c|c|c|c|}
\hline $\mathrm{C}$ & -3.91320100 & 0.32110300 & -3.06281600 \\
\hline $\mathrm{C}$ & -4.71397100 & 0.21180200 & -1.72937500 \\
\hline $\mathrm{N}$ & -4.24227700 & 1.32869400 & -0.96690800 \\
\hline C & -3.15651800 & 1.95906400 & -1.55166300 \\
\hline $\mathrm{N}$ & -2.91048800 & 1.31188800 & -2.75555200 \\
\hline $\mathrm{N}$ & -3.45752700 & -1.02275100 & -3.28446800 \\
\hline $\mathrm{H}$ & -4.52629900 & 0.65358500 & -3.91342500 \\
\hline $\mathrm{N}$ & -4.35063000 & -1.09809200 & -1.24920900 \\
\hline $\mathrm{H}$ & -5.80315300 & 0.28151600 & -1.86537600 \\
\hline $\mathrm{C}$ & -4.83292200 & 1.80537400 & 0.25301900 \\
\hline $\mathrm{O}$ & -2.58745300 & 2.93763300 & -1.13317000 \\
\hline $\mathrm{C}$ & -4.87188800 & -1.70223600 & -0.05691200 \\
\hline $\mathrm{C}$ & -3.68117200 & -1.85137200 & -2.19703400 \\
\hline $\mathrm{O}$ & -3.40757500 & -3.02589600 & -2.11804500 \\
\hline $\mathrm{N}$ & -4.28174400 & -1.24042300 & 1.16928700 \\
\hline $\mathrm{C}$ & -4.72688600 & -0.11219800 & 1.93111400 \\
\hline $\mathrm{C}$ & -3.19205600 & -1.87567500 & 1.73701900 \\
\hline $\mathrm{C}$ & -3.92626900 & -0.24235200 & 3.26285700 \\
\hline $\mathrm{N}$ & -4.33340300 & 1.19059600 & 1.45151800 \\
\hline $\mathrm{H}$ & -5.81702000 & -0.15766100 & 2.06920500 \\
\hline $\mathrm{N}$ & -2.93533100 & -1.24516500 & 2.94671700 \\
\hline $\mathrm{O}$ & -2.61786700 & -2.84122500 & 1.29054600 \\
\hline $\mathrm{N}$ & -3.45232300 & 1.09316000 & 3.49313800 \\
\hline $\mathrm{H}$ & -4.54281200 & -0.57442000 & 4.11100200 \\
\hline $\mathrm{C}$ & -3.68117200 & 1.93920100 & 2.41664600 \\
\hline $\mathrm{O}$ & -3.42382000 & 3.11758500 & 2.36262500 \\
\hline $\mathrm{C}$ & -2.14456800 & -1.91076800 & 3.94923900 \\
\hline $\mathrm{C}$ & -2.67493400 & 1.51914500 & 4.62533200 \\
\hline $\mathrm{N}$ & -1.28922700 & 1.12894900 & 4.62599800 \\
\hline $\mathrm{N}$ & -0.90037600 & -1.26338100 & 4.24781100 \\
\hline $\mathrm{C}$ & -0.30101000 & 1.93278700 & 4.07557500 \\
\hline $\mathrm{C}$ & -0.76960600 & -0.11448600 & 5.11441100 \\
\hline $\mathrm{C}$ & 0.76960600 & 0.11448600 & 5.11441100 \\
\hline $\mathrm{N}$ & 0.90037600 & 1.26338100 & 4.24781100 \\
\hline C & 0.30101000 & -1.93278700 & 4.07557500 \\
\hline $\mathrm{N}$ & 1.28922700 & -1.12894900 & 4.62599800 \\
\hline $\mathrm{C}$ & 2.14456800 & 1.91076800 & 3.94923900 \\
\hline C & 2.67493400 & -1.51914500 & 4.62533200 \\
\hline $\mathrm{O}$ & 0.45267600 & -3.03001900 & 3.59575900 \\
\hline $\mathrm{O}$ & -0.45267600 & 3.03001900 & 3.59575900 \\
\hline $\mathrm{N}$ & 2.93533100 & 1.24516500 & 2.94671700 \\
\hline $\mathrm{N}$ & 3.45232300 & -1.09316000 & 3.49313800 \\
\hline $\mathrm{C}$ & 3.19205600 & 1.87567500 & 1.73701900 \\
\hline $\mathrm{C}$ & 3.92626900 & 0.24235200 & 3.26285700 \\
\hline $\mathrm{C}$ & 4.72688600 & 0.11219800 & 1.93111400 \\
\hline $\mathrm{N}$ & 4.28174400 & 1.24042300 & 1.16928700 \\
\hline $\mathrm{O}$ & 2.61786700 & 2.84122500 & 1.29054600 \\
\hline C & 4.87188800 & 1.70223600 & -0.05691200 \\
\hline $\mathrm{N}$ & 4.33340300 & -1.19059600 & 1.45151800 \\
\hline $\mathrm{C}$ & 3.68117200 & -1.93920100 & 2.41664600 \\
\hline $\mathrm{O}$ & 3.42382000 & -3.11758500 & 2.3626250 \\
\hline
\end{tabular}




\begin{tabular}{|c|c|c|c|}
\hline $\mathrm{C}$ & 4.83292200 & -1.80537400 & 0.25301900 \\
\hline $\mathrm{N}$ & 4.35063000 & 1.09809200 & -1.24920900 \\
\hline $\mathrm{N}$ & 4.24227700 & -1.32869400 & -0.96690800 \\
\hline C & 3.68117200 & 1.85137200 & -2.19703400 \\
\hline C & 4.71397100 & -0.21180200 & -1.72937500 \\
\hline C & 3.91320100 & -0.32110300 & -3.06281600 \\
\hline $\mathrm{N}$ & 3.45752700 & 1.02275100 & -3.28446800 \\
\hline $\mathrm{O}$ & 3.40757500 & 3.02589600 & -2.11804500 \\
\hline C & 2.69880000 & 1.46870500 & -4.42171000 \\
\hline $\mathrm{N}$ & 2.91048800 & -1.31188800 & -2.75555200 \\
\hline $\mathrm{C}$ & 3.15651800 & -1.95906400 & -1.55166300 \\
\hline $\mathrm{O}$ & 2.58745300 & -2.93763300 & -1.13317000 \\
\hline C & 2.10777600 & -1.95488900 & -3.76066600 \\
\hline $\mathrm{N}$ & 1.30720700 & 1.10735500 & -4.43566600 \\
\hline $\mathrm{N}$ & 0.87640100 & -1.27977500 & -4.05036700 \\
\hline $\mathrm{C}$ & 0.76683600 & -0.12888900 & -4.91756500 \\
\hline $\mathrm{C}$ & -0.33389600 & -1.93506400 & -3.89796800 \\
\hline C & -0.76683600 & 0.12888900 & -4.91756500 \\
\hline $\mathrm{N}$ & -1.30720700 & -1.10735500 & -4.43566600 \\
\hline C & 0.33389600 & 1.93506400 & -3.89796800 \\
\hline $\mathrm{N}$ & -0.87640100 & 1.27977500 & -4.05036700 \\
\hline $\mathrm{O}$ & -0.50516100 & -3.04098000 & -3.44323500 \\
\hline $\mathrm{O}$ & 0.50516100 & 3.04098000 & -3.44323500 \\
\hline C & -2.69880000 & -1.46870500 & -4.42171000 \\
\hline C & -2.10777600 & 1.95488900 & -3.76066600 \\
\hline $\mathrm{H}$ & 3.13883300 & -1.13645300 & 5.54246900 \\
\hline $\mathrm{H}$ & 2.70531800 & -2.61120900 & 4.61977800 \\
\hline $\mathrm{H}$ & 1.89346800 & 2.90397300 & 3.56678500 \\
\hline $\mathrm{H}$ & 2.74421100 & 2.00498000 & 4.86862900 \\
\hline $\mathrm{H}$ & 5.95572200 & 1.53245900 & 0.00686600 \\
\hline $\mathrm{H}$ & 4.66857800 & 2.77233800 & -0.14557300 \\
\hline $\mathrm{H}$ & 5.92006000 & -1.65969900 & 0.18122300 \\
\hline $\mathrm{H}$ & 4.60703900 & -2.87095500 & 0.34340400 \\
\hline $\mathrm{H}$ & 3.16589000 & 1.07964900 & -5.33452000 \\
\hline $\mathrm{H}$ & 2.74766000 & 2.56001800 & -4.41258100 \\
\hline $\mathrm{H}$ & 2.70308300 & -2.05605300 & -4.68243000 \\
\hline $\mathrm{H}$ & 1.83746700 & -2.94457600 & -3.38201000 \\
\hline $\mathrm{H}$ & -1.83746700 & 2.94457600 & -3.38201000 \\
\hline $\mathrm{H}$ & -2.70308300 & 2.05605300 & -4.68243000 \\
\hline $\mathrm{H}$ & -2.74766000 & -2.56001800 & -4.41258100 \\
\hline $\mathrm{H}$ & -3.16589000 & -1.07964900 & -5.33452000 \\
\hline $\mathrm{H}$ & -5.92006000 & 1.65969900 & 0.18122300 \\
\hline $\mathrm{H}$ & -4.60703900 & 2.87095500 & 0.34340400 \\
\hline $\mathrm{H}$ & -4.66857800 & -2.77233800 & -0.14557300 \\
\hline $\mathrm{H}$ & -5.95572200 & -1.53245900 & 0.00686600 \\
\hline $\mathrm{H}$ & -1.18414400 & -0.33011600 & 6.11003200 \\
\hline $\mathrm{H}$ & 1.18414400 & 0.33011600 & 6.11003200 \\
\hline $\mathrm{H}$ & 5.81702000 & 0.15766100 & 2.06920500 \\
\hline $\mathrm{H}$ & 4.54281200 & 0.57442000 & 4.11100200 \\
\hline $\mathrm{H}$ & 5.80315300 & -0.28151600 & -1.86537600 \\
\hline $\mathrm{H}$ & 4.52629900 & -0.65358500 & -3.91342500 \\
\hline $\mathrm{H}$ & -1.17621400 & 0.35477700 & -5.91327100 \\
\hline $\mathrm{H}$ & -2.74421100 & -2.00498000 & 4.86862900 \\
\hline $\mathrm{H}$ & -1.89346800 & -2.90397300 & 3.56678500 \\
\hline $\mathrm{H}$ & -2.70531800 & 2.61120900 & 4.61977800 \\
\hline $\mathrm{H}$ & -3.13883300 & 1.13645300 & 5.54246900 \\
\hline $\mathrm{H}$ & 1.17621400 & -0.35477700 & -5.91327100 \\
\hline $\mathrm{Br}$ & -0.61398700 & -0.96746800 & -0.71911800 \\
\hline $\mathrm{Br}$ & 0.61398700 & 0.96746800 & -0.71911800 \\
\hline
\end{tabular}




\begin{tabular}{|c|c|c|c|}
\hline \multicolumn{4}{|c|}{$\mathrm{CS}_{2} @ \mathrm{CB}[6]$} \\
\hline $\mathrm{C}$ & 4.85412200 & -0.50362300 & 0.03203200 \\
\hline $\mathrm{C}$ & 4.78535300 & 1.05340500 & -0.03365800 \\
\hline $\mathrm{N}$ & 4.16991800 & 1.41181600 & 1.21196900 \\
\hline $\mathrm{C}$ & 3.79485200 & 0.31175200 & 1.96798500 \\
\hline $\mathrm{N}$ & 4.10545800 & -0.81776400 & 1.22285800 \\
\hline $\mathrm{N}$ & 4.28017900 & -0.91600900 & -1.21610700 \\
\hline $\mathrm{H}$ & 5.88081400 & -0.89064800 & 0.12062900 \\
\hline $\mathrm{N}$ & 4.02183300 & 1.29877300 & -1.23049100 \\
\hline $\mathrm{H}$ & 5.77412400 & 1.53056000 & -0.11333400 \\
\hline $\mathrm{C}$ & 4.04769000 & 2.75531100 & 1.70970600 \\
\hline $\mathrm{O}$ & 3.34205300 & 0.33204200 & 3.08716100 \\
\hline $\mathrm{C}$ & 3.87615200 & 2.60097300 & -1.82396400 \\
\hline $\mathrm{C}$ & 3.81277200 & 0.14591300 & -1.97529500 \\
\hline $\mathrm{O}$ & 3.36738200 & 0.08464700 & -3.09588200 \\
\hline $\mathrm{N}$ & 2.82176500 & 3.40403200 & -1.26824400 \\
\hline $\mathrm{C}$ & 2.94312400 & 4.20895700 & -0.07945400 \\
\hline $\mathrm{C}$ & 1.69315400 & 3.70670300 & -2.01205100 \\
\hline $\mathrm{C}$ & 1.59671500 & 5.00608000 & -0.04772500 \\
\hline $\mathrm{N}$ & 2.94813100 & 3.51758500 & 1.18139500 \\
\hline $\mathrm{H}$ & 3.82959200 & 4.85652600 & -0.15359400 \\
\hline $\mathrm{N}$ & 0.96783600 & 4.63940100 & -1.28630400 \\
\hline $\mathrm{O}$ & 1.42148400 & 3.28570300 & -3.11051200 \\
\hline $\mathrm{N}$ & 0.97229200 & 4.55033600 & 1.16985700 \\
\hline $\mathrm{H}$ & 1.73913500 & 6.09602000 & -0.00863700 \\
\hline $\mathrm{C}$ & 1.81568100 & 3.76198100 & 1.94039600 \\
\hline $\mathrm{O}$ & 1.62377200 & 3.40513200 & 3.07756600 \\
\hline $\mathrm{C}$ & -0.31782800 & 5.09898900 & -1.73638700 \\
\hline $\mathrm{C}$ & -0.23219700 & 5.10604200 & 1.73274800 \\
\hline $\mathrm{N}$ & -1.45325700 & 4.49574200 & 1.28352800 \\
\hline $\mathrm{N}$ & -1.44668900 & 4.40324500 & -1.17352200 \\
\hline $\mathrm{C}$ & -2.05939800 & 3.48142900 & 2.00955900 \\
\hline $\mathrm{C}$ & -2.12095500 & 4.78090700 & 0.04386500 \\
\hline $\mathrm{C}$ & -3.35956800 & 3.82524100 & 0.07673200 \\
\hline $\mathrm{N}$ & -3.14143100 & 3.04137200 & 1.26574000 \\
\hline $\mathrm{C}$ & -2.18622300 & 3.51455700 & -1.94153400 \\
\hline $\mathrm{N}$ & -3.28148900 & 3.13619200 & -1.18273500 \\
\hline $\mathrm{C}$ & -4.09599900 & 2.12459400 & 1.82547600 \\
\hline $\mathrm{C}$ & -4.29255200 & 2.26372400 & -1.71700900 \\
\hline $\mathrm{O}$ & -1.95176900 & 3.18141700 & -3.07792700 \\
\hline $\mathrm{O}$ & -1.73925700 & 3.09910100 & 3.10920900 \\
\hline $\mathrm{N}$ & -4.10545800 & 0.81776400 & 1.22285800 \\
\hline $\mathrm{N}$ & -4.28017900 & 0.91600900 & -1.21610700 \\
\hline $\mathrm{C}$ & -3.79485200 & -0.31175200 & 1.96798500 \\
\hline $\mathrm{C}$ & -4.85412200 & 0.50362300 & 0.03203200 \\
\hline $\mathrm{C}$ & -4.78535300 & -1.05340500 & -0.03365800 \\
\hline $\mathrm{N}$ & -4.16991800 & -1.41181600 & 1.21196900 \\
\hline $\mathrm{O}$ & -3.34205300 & -0.33204200 & 3.08716100 \\
\hline $\mathrm{C}$ & -4.04769000 & -2.75531100 & 1.70970600 \\
\hline $\mathrm{N}$ & -4.02183300 & -1.29877300 & -1.23049100 \\
\hline $\mathrm{C}$ & -3.81277200 & -0.14591300 & -1.97529500 \\
\hline $\mathrm{O}$ & -3.36738200 & -0.08464700 & -3.09588200 \\
\hline $\mathrm{C}$ & -3.87615200 & -2.60097300 & -1.82396400 \\
\hline $\mathrm{N}$ & -2.94813100 & -3.51758500 & 1.18139500 \\
\hline $\mathrm{N}$ & -2.82176500 & -3.40403200 & -1.26824400 \\
\hline $\mathrm{C}$ & -1.81568100 & -3.76198100 & 1.94039600 \\
\hline C & -2.94312400 & -4.20895700 & -0.07945400 \\
\hline
\end{tabular}




\begin{tabular}{|c|c|c|c|}
\hline C & -1.59671500 & -5.00608000 & -0.04772500 \\
\hline $\mathrm{N}$ & -0.97229200 & -4.55033600 & 1.16985700 \\
\hline $\mathrm{O}$ & -1.62377200 & -3.40513200 & 3.07756600 \\
\hline $\mathrm{C}$ & 0.23219700 & -5.10604200 & 1.73274800 \\
\hline $\mathrm{N}$ & -0.96783600 & -4.63940100 & -1.28630400 \\
\hline C & -1.69315400 & -3.70670300 & -2.01205100 \\
\hline $\mathrm{O}$ & -1.42148400 & -3.28570300 & -3.11051200 \\
\hline C & 0.31782800 & -5.09898900 & -1.73638700 \\
\hline $\mathrm{N}$ & 1.45325700 & -4.49574200 & 1.28352800 \\
\hline $\mathrm{N}$ & 1.44668900 & -4.40324500 & -1.17352200 \\
\hline $\mathrm{C}$ & 2.12095500 & -4.78090700 & 0.04386500 \\
\hline C & 2.18622300 & -3.51455700 & -1.94153400 \\
\hline C & 3.35956800 & -3.82524100 & 0.07673200 \\
\hline $\mathrm{N}$ & 3.28148900 & -3.13619200 & -1.18273500 \\
\hline C & 2.05939800 & -3.48142900 & 2.00955900 \\
\hline $\mathrm{N}$ & 3.14143100 & -3.04137200 & 1.26574000 \\
\hline $\mathrm{O}$ & 1.95176900 & -3.18141700 & -3.07792700 \\
\hline $\mathrm{O}$ & 1.73925700 & -3.09910100 & 3.10920900 \\
\hline C & 4.29255200 & -2.26372400 & -1.71700900 \\
\hline C & 4.09599900 & -2.12459400 & 1.82547600 \\
\hline $\mathrm{H}$ & -5.28077900 & 2.70240000 & -1.52065300 \\
\hline $\mathrm{H}$ & -4.11788400 & 2.20271700 & -2.79427200 \\
\hline $\mathrm{H}$ & -3.82970300 & 1.98949600 & 2.87730400 \\
\hline $\mathrm{H}$ & -5.10312700 & 2.56258500 & 1.74682500 \\
\hline $\mathrm{H}$ & -4.98329500 & -3.29366000 & 1.50503700 \\
\hline $\mathrm{H}$ & -3.88684300 & -2.67980200 & 2.78818000 \\
\hline $\mathrm{H}$ & -4.82758900 & -3.14727600 & -1.73357200 \\
\hline $\mathrm{H}$ & -3.63519300 & -2.44583600 & -2.87907500 \\
\hline $\mathrm{H}$ & 0.26996700 & -6.18146000 & 1.51393000 \\
\hline $\mathrm{H}$ & 0.17825800 & -4.94511500 & 2.81255000 \\
\hline $\mathrm{H}$ & 0.40697500 & -6.17127600 & -1.51699000 \\
\hline $\mathrm{H}$ & 0.35101800 & -4.93358700 & -2.81637900 \\
\hline $\mathrm{H}$ & 3.82970300 & -1.98949600 & 2.87730400 \\
\hline $\mathrm{H}$ & 5.10312700 & -2.56258500 & 1.74682500 \\
\hline $\mathrm{H}$ & 4.11788400 & -2.20271700 & -2.79427200 \\
\hline $\mathrm{H}$ & 5.28077900 & -2.70240000 & -1.52065300 \\
\hline $\mathrm{H}$ & 4.98329500 & 3.29366000 & 1.50503700 \\
\hline $\mathrm{H}$ & 3.88684300 & 2.67980200 & 2.78818000 \\
\hline $\mathrm{H}$ & 3.63519300 & 2.44583600 & -2.87907500 \\
\hline $\mathrm{H}$ & 4.82758900 & 3.14727600 & -1.73357200 \\
\hline $\mathrm{H}$ & -2.39625700 & 5.84491800 & 0.00318400 \\
\hline $\mathrm{H}$ & -4.31775400 & 4.36122700 & 0.15142200 \\
\hline $\mathrm{H}$ & -5.77412400 & -1.53056000 & -0.11333400 \\
\hline $\mathrm{H}$ & -5.88081400 & 0.89064800 & 0.12062900 \\
\hline $\mathrm{H}$ & -3.82959200 & -4.85652600 & -0.15359400 \\
\hline $\mathrm{H}$ & -1.73913500 & -6.09602000 & -0.00863700 \\
\hline $\mathrm{H}$ & 4.31775400 & -4.36122700 & 0.15142200 \\
\hline $\mathrm{H}$ & -0.40697500 & 6.17127600 & -1.51699000 \\
\hline $\mathrm{H}$ & -0.35101800 & 4.93358700 & -2.81637900 \\
\hline $\mathrm{H}$ & -0.17825800 & 4.94511500 & 2.81255000 \\
\hline $\mathrm{H}$ & -0.26996700 & 6.18146000 & 1.51393000 \\
\hline $\mathrm{H}$ & 2.39625700 & -5.84491800 & 0.00318400 \\
\hline C & 0.00000000 & 0.00000000 & 0.02218900 \\
\hline S & 0.00000000 & 1.55258800 & 0.02248800 \\
\hline S & 0.00000000 & -1.55258800 & 0.02248800 \\
\hline
\end{tabular}




\begin{tabular}{|c|c|c|c|}
\hline $\mathrm{C}$ & -0.10407300 & 5.06493900 & 0.78202000 \\
\hline $\mathrm{C}$ & -0.10407300 & 5.06493900 & -0.78202000 \\
\hline $\mathrm{N}$ & -1.27821300 & 4.30454100 & -1.11355600 \\
\hline $\mathrm{C}$ & -1.99567500 & 3.89823800 & 0.00000000 \\
\hline $\mathrm{N}$ & -1.27821300 & 4.30454100 & 1.11355600 \\
\hline $\mathrm{N}$ & 1.16757000 & 4.47887000 & 1.11343200 \\
\hline $\mathrm{H}$ & -0.17656600 & 6.07310500 & 1.21703500 \\
\hline $\mathrm{N}$ & 1.16757000 & 4.47887000 & -1.11343200 \\
\hline $\mathrm{H}$ & -0.17656600 & 6.07310500 & -1.21703500 \\
\hline $\mathrm{C}$ & -1.80408800 & 4.14613400 & -2.44106100 \\
\hline $\mathrm{O}$ & -3.07250400 & 3.35190900 & 0.00000000 \\
\hline $\mathrm{C}$ & 1.70783200 & 4.37911300 & -2.44209000 \\
\hline $\mathrm{C}$ & 1.94398100 & 4.19979500 & 0.00000000 \\
\hline $\mathrm{O}$ & 3.09883100 & 3.84662100 & 0.00000000 \\
\hline $\mathrm{N}$ & 1.24196200 & 3.26120600 & -3.21754600 \\
\hline $\mathrm{C}$ & 0.01416700 & 3.21659600 & -3.95936200 \\
\hline $\mathrm{C}$ & 1.96427400 & 2.08188000 & -3.29632100 \\
\hline $\mathrm{C}$ & 0.10341200 & 1.85649700 & -4.72004800 \\
\hline $\mathrm{N}$ & -1.20705400 & 3.08199600 & -3.20329700 \\
\hline $\mathrm{H}$ & -0.04875900 & 4.08673200 & -4.62976200 \\
\hline $\mathrm{N}$ & 1.23887700 & 1.21611300 & -4.10198100 \\
\hline $\mathrm{O}$ & 3.04965200 & 1.87116800 & -2.81036300 \\
\hline $\mathrm{N}$ & -1.18177200 & 1.26209900 & -4.49078900 \\
\hline $\mathrm{H}$ & 0.27676700 & 1.97359200 & -5.80032200 \\
\hline $\mathrm{C}$ & -1.96150800 & 1.98852500 & -3.60300100 \\
\hline $\mathrm{O}$ & -3.10271700 & 1.74875800 & -3.29094500 \\
\hline $\mathrm{C}$ & 1.83893100 & 0.00660600 & -4.59204100 \\
\hline $\mathrm{C}$ & -1.65727900 & 0.04457800 & -5.09157800 \\
\hline $\mathrm{N}$ & -1.20281400 & -1.17706000 & -4.48228500 \\
\hline $\mathrm{N}$ & 1.22153200 & -1.20524900 & -4.12711800 \\
\hline $\mathrm{C}$ & -2.01879500 & -1.90864100 & -3.63121500 \\
\hline $\mathrm{C}$ & 0.06067700 & -1.81233200 & -4.73005500 \\
\hline $\mathrm{C}$ & -0.05731800 & -3.17247600 & -3.96669200 \\
\hline $\mathrm{N}$ & -1.31310900 & -3.04520500 & -3.27614600 \\
\hline $\mathrm{C}$ & 1.86366500 & -2.04110700 & -3.23711500 \\
\hline $\mathrm{N}$ & 1.12864800 & -3.20442900 & -3.15370100 \\
\hline $\mathrm{C}$ & -1.88939800 & -4.05210900 & -2.42940000 \\
\hline $\mathrm{C}$ & 1.61834900 & -4.35868500 & -2.44047900 \\
\hline $\mathrm{O}$ & 2.91824900 & -1.81793800 & -2.67777000 \\
\hline $\mathrm{O}$ & -3.15303600 & -1.63778000 & -3.31884700 \\
\hline $\mathrm{N}$ & -1.31065100 & -4.14695500 & -1.11604600 \\
\hline $\mathrm{N}$ & 1.11459000 & -4.52674500 & -1.11057400 \\
\hline $\mathrm{C}$ & -2.00620800 & -3.70731300 & 0.00000000 \\
\hline $\mathrm{C}$ & -0.20167100 & -4.99948300 & -0.78131400 \\
\hline $\mathrm{C}$ & -0.20167100 & -4.99948300 & 0.78131400 \\
\hline $\mathrm{N}$ & -1.31065100 & -4.14695500 & 1.11604600 \\
\hline $\mathrm{O}$ & -3.05918700 & -3.11643600 & 0.00000000 \\
\hline $\mathrm{C}$ & -1.88939800 & -4.05210900 & 2.42940000 \\
\hline $\mathrm{N}$ & 1.11459000 & -4.52674500 & 1.11057400 \\
\hline $\mathrm{C}$ & 1.90291300 & -4.28122000 & 0.00000000 \\
\hline $\mathrm{O}$ & 3.08336200 & -4.01890800 & 0.00000000 \\
\hline $\mathrm{C}$ & 1.61834900 & -4.35868500 & 2.44047900 \\
\hline $\mathrm{N}$ & -1.31310900 & -3.04520500 & 3.27614600 \\
\hline $\mathrm{N}$ & 1.12864800 & -3.20442900 & 3.15370100 \\
\hline $\mathrm{C}$ & -2.01879500 & -1.90864100 & 3.63121500 \\
\hline $\mathrm{C}$ & -0.05731800 & -3.17247600 & 3.96669200 \\
\hline $\mathrm{C}$ & 0.06067700 & -1.81233200 & 4.73005500 \\
\hline $\mathrm{N}$ & -1.20281400 & -1.17706000 & 4.48228500 \\
\hline $\mathrm{O}$ & -3.15303600 & -1.63778000 & 3.31884700 \\
\hline $\mathrm{C}$ & -1.65727900 & 0.04457800 & 5.09157800 \\
\hline $\mathrm{N}$ & 1.22153200 & -1.20524900 & 4.12711800 \\
\hline
\end{tabular}




\begin{tabular}{|c|c|c|c|}
\hline $\mathrm{C}$ & 1.86366500 & -2.04110700 & 3.23711500 \\
\hline $\mathrm{O}$ & 2.91824900 & -1.81793800 & 2.67777000 \\
\hline $\mathrm{C}$ & 1.83893100 & 0.00660600 & 4.59204100 \\
\hline $\mathrm{N}$ & -1.18177200 & 1.26209900 & 4.49078900 \\
\hline $\mathrm{N}$ & 1.23887700 & 1.21611300 & 4.10198100 \\
\hline $\mathrm{C}$ & 0.10341200 & 1.85649700 & 4.72004800 \\
\hline $\mathrm{C}$ & 1.96427400 & 2.08188000 & 3.29632100 \\
\hline $\mathrm{C}$ & 0.01416700 & 3.21659600 & 3.95936200 \\
\hline $\mathrm{N}$ & 1.24196200 & 3.26120600 & 3.21754600 \\
\hline $\mathrm{C}$ & -1.96150800 & 1.98852500 & 3.60300100 \\
\hline $\mathrm{N}$ & -1.20705400 & 3.08199600 & 3.20329700 \\
\hline $\mathrm{O}$ & 3.04965200 & 1.87116800 & 2.81036300 \\
\hline $\mathrm{O}$ & -3.10271700 & 1.74875800 & 3.29094500 \\
\hline $\mathrm{C}$ & 1.70783200 & 4.37911300 & 2.44209000 \\
\hline $\mathrm{C}$ & -1.80408800 & 4.14613400 & 2.44106100 \\
\hline $\mathrm{H}$ & 1.38403700 & -5.25023700 & -3.03824000 \\
\hline $\mathrm{H}$ & 2.70125200 & -4.24825300 & -2.34355900 \\
\hline $\mathrm{H}$ & -2.94253100 & -3.78619600 & -2.30460000 \\
\hline $\mathrm{H}$ & -1.80556400 & -5.03210500 & -2.92160100 \\
\hline $\mathrm{H}$ & -1.80556400 & -5.03210500 & 2.92160100 \\
\hline $\mathrm{H}$ & -2.94253100 & -3.78619600 & 2.30460000 \\
\hline $\mathrm{H}$ & 1.38403700 & -5.25023700 & 3.03824000 \\
\hline $\mathrm{H}$ & 2.70125200 & -4.24825300 & 2.34355900 \\
\hline $\mathrm{H}$ & -1.37118500 & 0.04024400 & 6.15169400 \\
\hline $\mathrm{H}$ & -2.74609100 & 0.05421600 & 4.99725800 \\
\hline $\mathrm{H}$ & 1.82319700 & 0.01134900 & 5.69252800 \\
\hline $\mathrm{H}$ & 2.87196400 & -0.00158300 & 4.23398400 \\
\hline $\mathrm{H}$ & -2.86558100 & 3.90459400 & 2.33975600 \\
\hline $\mathrm{H}$ & -1.68514700 & 5.09580000 & 2.98335100 \\
\hline $\mathrm{H}$ & 2.78841800 & 4.26116600 & 2.32876900 \\
\hline $\mathrm{H}$ & 1.48574300 & 5.30261100 & 2.99355900 \\
\hline $\mathrm{H}$ & -1.68514700 & 5.09580000 & -2.98335100 \\
\hline $\mathrm{H}$ & -2.86558100 & 3.90459400 & -2.33975600 \\
\hline $\mathrm{H}$ & 2.78841800 & 4.26116600 & -2.32876900 \\
\hline $\mathrm{H}$ & 1.48574300 & 5.30261100 & -2.99355900 \\
\hline $\mathrm{H}$ & 0.21843600 & -1.93124800 & -5.81195600 \\
\hline $\mathrm{H}$ & -0.07419700 & -4.04729500 & -4.63298600 \\
\hline $\mathrm{H}$ & -0.35822200 & -5.99866900 & 1.21585200 \\
\hline $\mathrm{H}$ & -0.35822200 & -5.99866900 & -1.21585200 \\
\hline $\mathrm{H}$ & -0.07419700 & -4.04729500 & 4.63298600 \\
\hline $\mathrm{H}$ & 0.21843600 & -1.93124800 & 5.81195600 \\
\hline $\mathrm{H}$ & -0.04875900 & 4.08673200 & 4.62976200 \\
\hline $\mathrm{H}$ & 1.82319700 & 0.01134900 & -5.69252800 \\
\hline $\mathrm{H}$ & 2.87196400 & -0.00158300 & -4.23398400 \\
\hline $\mathrm{H}$ & -2.74609100 & 0.05421600 & -4.99725800 \\
\hline $\mathrm{H}$ & -1.37118500 & 0.04024400 & -6.15169400 \\
\hline $\mathrm{H}$ & 0.27676700 & 1.97359200 & 5.80032200 \\
\hline $\mathrm{H}$ & 2.26015400 & -1.44315700 & -0.75372600 \\
\hline $\mathrm{H}$ & 2.26015400 & -1.44315700 & 0.75372600 \\
\hline $\mathrm{O}$ & 1.65740600 & -1.42351700 & 0.00000000 \\
\hline
\end{tabular}

\section{HF@CB[6]}

$\begin{array}{lllr}\mathrm{C} & -1.37991700 & 4.74232600 & -0.18066700 \\ \mathrm{C} & -2.82682100 & 4.19092200 & -0.01074100 \\ \mathrm{~N} & -2.72484300 & 3.42737300 & 1.21009600 \\ \mathrm{C} & -1.57743300 & 3.73063300 & 1.92771900\end{array}$




\begin{tabular}{|c|c|c|c|}
\hline $\mathrm{N}$ & -0.78862600 & 4.52456700 & 1.10654800 \\
\hline $\mathrm{N}$ & -0.87080400 & 3.94565500 & -1.27442600 \\
\hline $\mathrm{H}$ & -1.34524600 & 5.81207100 & -0.43630900 \\
\hline $\mathrm{N}$ & -3.04614200 & 3.45725500 & -1.22295400 \\
\hline $\mathrm{H}$ & -3.58705400 & 4.97933000 & 0.09235700 \\
\hline C & -3.83442800 & 2.76304700 & 1.83653300 \\
\hline $\mathrm{O}$ & -1.33677300 & 3.41989100 & 3.06907900 \\
\hline C & -4.30069800 & 2.88689300 & -1.64241100 \\
\hline C & -1.88551900 & 3.29210100 & -1.96117900 \\
\hline $\mathrm{O}$ & -1.78696200 & 2.73766600 & -3.02954000 \\
\hline $\mathrm{N}$ & -4.59105200 & 1.57332600 & -1.13704000 \\
\hline C & -5.06651600 & 1.26964800 & 0.18494100 \\
\hline C & -4.38933900 & 0.44439400 & -1.91646100 \\
\hline C & -5.24106100 & -0.28404600 & 0.15358800 \\
\hline $\mathrm{N}$ & -4.14851200 & 1.47591300 & 1.27664100 \\
\hline $\mathrm{H}$ & -6.00727200 & 1.80631200 & 0.37707300 \\
\hline $\mathrm{N}$ & -4.66666900 & -0.65347600 & -1.11714600 \\
\hline $\mathrm{O}$ & -4.09381100 & 0.42645900 & -3.08691900 \\
\hline $\mathrm{N}$ & -4.55041300 & -0.71605200 & 1.33483300 \\
\hline $\mathrm{H}$ & -6.29298800 & -0.60296300 & 0.19704300 \\
\hline $\mathrm{C}$ & -3.90623400 & 0.31559200 & 1.99738300 \\
\hline $\mathrm{O}$ & -3.30266900 & 0.23107000 & 3.03955600 \\
\hline C & -4.70912600 & -1.97699100 & -1.68225200 \\
\hline C & -4.46692900 & -2.07246800 & 1.80102900 \\
\hline $\mathrm{N}$ & -3.43838200 & -2.86570800 & 1.18001200 \\
\hline $\mathrm{N}$ & -3.64861000 & -2.85363700 & -1.26801100 \\
\hline C & -2.38770000 & -3.36585600 & 1.93923900 \\
\hline C & -3.62149700 & -3.60498600 & -0.04796400 \\
\hline C & -2.29552100 & -4.41733500 & -0.15973700 \\
\hline $\mathrm{N}$ & -1.70802600 & -4.27165900 & 1.14072600 \\
\hline C & -2.45338400 & -2.92413700 & -1.96847000 \\
\hline $\mathrm{N}$ & -1.61653600 & -3.77187800 & -1.25898300 \\
\hline C & -0.55959600 & -4.99293500 & 1.62226900 \\
\hline C & -0.42363800 & -4.28931100 & -1.87382500 \\
\hline $\mathrm{O}$ & -2.21514800 & -2.39522000 & -3.02774600 \\
\hline $\mathrm{O}$ & -2.15046600 & -3.10981900 & 3.09496200 \\
\hline $\mathrm{N}$ & 0.72032900 & -4.49589900 & 1.19917800 \\
\hline $\mathrm{N}$ & 0.79630500 & -3.95157800 & -1.19247300 \\
\hline C & 1.51223000 & -3.71370100 & 2.02859200 \\
\hline $\mathrm{C}$ & 1.31585500 & -4.72132700 & -0.08634000 \\
\hline $\mathrm{C}$ & 2.75744500 & -4.15437300 & 0.07985500 \\
\hline $\mathrm{N}$ & 2.67152200 & -3.43679900 & 1.32600100 \\
\hline $\mathrm{O}$ & 1.26363300 & -3.39735200 & 3.16741700 \\
\hline C & 3.76178000 & -2.71963500 & 1.91987200 \\
\hline $\mathrm{N}$ & 2.94945900 & -3.37164100 & -1.10462900 \\
\hline C & 1.79581700 & -3.25422100 & -1.86187400 \\
\hline $\mathrm{O}$ & 1.69723300 & -2.71720600 & -2.93897400 \\
\hline C & 4.21855500 & -2.87494900 & -1.56547600 \\
\hline $\mathrm{N}$ & 4.05025200 & -1.45026600 & 1.30510100 \\
\hline $\mathrm{N}$ & 4.59914800 & -1.57379400 & -1.08887600 \\
\hline C & 3.70435700 & -0.27008800 & 1.94261300 \\
\hline $\mathrm{C}$ & 5.01378100 & -1.25698600 & 0.25303500 \\
\hline C & 5.19775000 & 0.29811000 & 0.21532800 \\
\hline $\mathrm{N}$ & 4.33890400 & 0.76323300 & 1.27053700 \\
\hline $\mathrm{O}$ & 3.03866000 & -0.16491600 & 2.94440300 \\
\hline $\mathrm{C}$ & 4.36823500 & 2.08978900 & 1.82799700 \\
\hline $\mathrm{N}$ & 4.82171200 & 0.63764600 & -1.13136900 \\
\hline $\mathrm{C}$ & 4.44072600 & -0.45809000 & -1.88444700 \\
\hline $\mathrm{O}$ & 4.12450700 & -0.44300600 & -3.05255100 \\
\hline C & 4.68081100 & 1.96517600 & -1.64780400 \\
\hline $\mathrm{N}$ & 3.42623200 & 3.00838500 & 1.25241100 \\
\hline
\end{tabular}




$\begin{array}{lrrr} & & & \\ \mathrm{N} & 3.54191600 & 2.71407800 & -1.17078300 \\ \mathrm{C} & 3.55922900 & 3.60922600 & -0.04844100 \\ \mathrm{C} & 2.33452500 & 2.72065800 & -1.83412500 \\ \mathrm{~N} & 2.22542100 & 4.40450400 & -0.19775900 \\ \mathrm{C} & 1.53761400 & 3.68366300 & -1.24554200 \\ \mathrm{~N} & 2.34326300 & 3.49056300 & 1.96654500 \\ \mathrm{O} & 1.64629400 & 4.33569000 & 1.11283900 \\ \mathrm{O} & 2.04664000 & 2.04701600 & -2.80430500 \\ \mathrm{C} & 2.09123700 & 3.27753500 & 3.12767900 \\ \mathrm{C} & 0.38114000 & 4.20025500 & -1.92933700 \\ \mathrm{H} & 0.47607200 & 5.05206400 & 1.54442800 \\ \mathrm{H} & -0.50431500 & -5.38601000 & -1.95298700 \\ \mathrm{H} & -0.36440900 & -3.84921200 & -2.87312700 \\ \mathrm{H} & -0.57981700 & -4.92092900 & 2.71251700 \\ \mathrm{H} & -0.65110300 & -6.04236100 & 1.31267000 \\ \mathrm{H} & 4.67292300 & -3.33595700 & 1.88829100 \\ \mathrm{H} & 3.47766700 & -2.53036000 & 2.95862000 \\ \mathrm{H} & 4.99146600 & -3.60241800 & -1.28624600 \\ \mathrm{H} & 4.16050500 & -2.78922400 & -2.65330100 \\ \mathrm{H} & 5.38637700 & 2.48976100 & 1.71928700 \\ \mathrm{H} & 4.10898500 & 2.01476200 & 2.88752200 \\ \mathrm{H} & 5.58025500 & 2.54984700 & -1.41699400 \\ \mathrm{H} & 4.57875200 & 1.85355200 & -2.72988400 \\ \mathrm{H} & 0.47500700 & 5.01218900 & 2.63644800 \\ \mathrm{H} & 0.55387100 & 6.09378900 & 1.20624800 \\ \mathrm{H} & 0.32897700 & 3.70802200 & -2.90416300 \\ \mathrm{H} & 0.51280900 & 5.28575700 & -2.06671700 \\ \mathrm{H} & -4.72774700 & 3.40392700 & 1.78014900 \\ \mathrm{H} & -3.55566400 & 2.60631700 & 2.88225500 \\ \mathrm{H} & -4.26603700 & 2.79345000 & -2.73047500 \\ \mathrm{H} & -5.10384300 & 3.57430100 & -1.34962800 \\ \mathrm{H} & -4.51748400 & -4.23952400 & 0.02146300 \\ \mathrm{H} & -2.45453800 & -5.48307400 & -0.38281400 \\ \mathrm{H} & 3.53036700 & -4.93516700 & 0.14156000 \\ \mathrm{H} & 1.29298800 & -5.79508800 & -0.32702200 \\ \mathrm{H} & 5.94174900 & -1.79586400 & 0.49297100 \\ \mathrm{H} & 6.23269300 & 0.61573100 & 0.40723300 \\ \mathrm{H} & 2.37035200 & 5.45351000 & -0.49453200 \\ \mathrm{H} & -5.66763400 & -2.45276600 & -1.43597400 \\ -4.62033800 & -1.85281000 & -2.76441700 \\ \mathrm{H} & -4.22966000 & -2.03304100 & 2.86746600 \\ \mathrm{H} & 4.45637000 & -2.55271300 & 1.65215400 \\ \mathrm{H} & 1.86014000 & -0.13747100 & -1.14686800\end{array}$

\section{HCl@CB[6]}

$\begin{array}{lrrr}\mathrm{C} & -0.55967500 & 4.99045400 & -0.08852200 \\ \mathrm{C} & -2.07093200 & 4.62089600 & 0.03058500 \\ \mathrm{~N} & -2.11150400 & 3.83230700 & 1.23746400 \\ \mathrm{C} & -0.93532800 & 3.91506400 & 1.96747100 \\ \mathrm{~N} & -0.02147200 & 4.60302500 & 1.18283700 \\ \mathrm{~N} & -0.13369800 & 4.22630300 & -1.23641700 \\ \mathrm{H} & -0.38458900 & 6.06303000 & -0.26073000 \\ \mathrm{~N} & -2.34314800 & 3.93947100 & -1.20258500 \\ \mathrm{H} & -2.72910000 & 5.49727500 & 0.12638600\end{array}$




\begin{tabular}{|c|c|c|c|}
\hline $\mathrm{C}$ & -3.32453100 & 3.35900400 & 1.84646700 \\
\hline $\mathrm{O}$ & -0.75852300 & 3.52294800 & 3.09553300 \\
\hline C & -3.64294800 & 3.52458700 & -1.65955000 \\
\hline C & -1.20065000 & 3.69751200 & -1.94756000 \\
\hline $\mathrm{O}$ & -1.15130700 & 3.18305900 & -3.03930900 \\
\hline $\mathrm{N}$ & -4.11546500 & 2.26894200 & -1.14371300 \\
\hline $\mathrm{C}$ & -4.71264300 & 2.07177600 & 0.14793600 \\
\hline $\mathrm{C}$ & -4.07980100 & 1.11456300 & -1.90964800 \\
\hline $\mathrm{C}$ & -5.14739100 & 0.57087700 & 0.11621800 \\
\hline $\mathrm{N}$ & -3.83690700 & 2.13690000 & 1.28991700 \\
\hline $\mathrm{H}$ & -5.55528900 & 2.76676800 & 0.28039300 \\
\hline $\mathrm{N}$ & -4.58665000 & 0.09039700 & -1.12317500 \\
\hline $\mathrm{O}$ & -3.73454100 & 1.03162600 & -3.06370700 \\
\hline $\mathrm{N}$ & -4.59605500 & 0.04294100 & 1.33137100 \\
\hline $\mathrm{H}$ & -6.23915700 & 0.43519200 & 0.11005000 \\
\hline $\mathrm{C}$ & -3.82450200 & 0.96120400 & 2.02470100 \\
\hline $\mathrm{O}$ & -3.29740700 & 0.78947100 & 3.09729700 \\
\hline $\mathrm{C}$ & -4.87968100 & -1.19661400 & -1.69959600 \\
\hline $\mathrm{C}$ & -4.75632800 & -1.30595400 & 1.79672500 \\
\hline $\mathrm{N}$ & -3.87252800 & -2.26937000 & 1.19161400 \\
\hline $\mathrm{N}$ & -4.03576100 & -2.27204600 & -1.25962800 \\
\hline $\mathrm{C}$ & -2.91548900 & -2.91943200 & 1.95969700 \\
\hline $\mathrm{C}$ & -4.17698200 & -2.98661200 & -0.02396900 \\
\hline $\mathrm{C}$ & -3.04014500 & -4.05388700 & -0.09807900 \\
\hline $\mathrm{N}$ & -2.40939200 & -3.95307400 & 1.18743700 \\
\hline $\mathrm{C}$ & -2.90044200 & -2.63398200 & -1.96829700 \\
\hline $\mathrm{N}$ & -2.26846400 & -3.62947800 & -1.23996300 \\
\hline $\mathrm{C}$ & -1.38267900 & -4.83000300 & 1.68312200 \\
\hline $\mathrm{C}$ & -1.21269800 & -4.40648200 & -1.82934800 \\
\hline $\mathrm{O}$ & -2.56575600 & -2.20898700 & -3.04797700 \\
\hline $\mathrm{O}$ & -2.62550700 & -2.67281900 & 3.10536200 \\
\hline $\mathrm{N}$ & -0.04791900 & -4.55869800 & 1.22143000 \\
\hline $\mathrm{N}$ & 0.07203900 & -4.24338800 & -1.20687100 \\
\hline $\mathrm{C}$ & 0.86831600 & -3.87466400 & 2.00594600 \\
\hline $\mathrm{C}$ & 0.49613100 & -4.97577200 & -0.03895800 \\
\hline $\mathrm{C}$ & 2.00676400 & -4.60117900 & 0.07813800 \\
\hline $\mathrm{N}$ & 2.04981800 & -3.81174800 & 1.28354200 \\
\hline $\mathrm{O}$ & 0.68911300 & -3.47121600 & 3.12987500 \\
\hline $\mathrm{C}$ & 3.26258200 & -3.33779000 & 1.89159600 \\
\hline $\mathrm{N}$ & 2.27503700 & -3.91985700 & -1.15496400 \\
\hline $\mathrm{C}$ & 1.13416700 & -3.69754900 & -1.90943200 \\
\hline $\mathrm{O}$ & 1.08862900 & -3.18799700 & -3.00412300 \\
\hline $\mathrm{C}$ & 3.57349600 & -3.51157600 & -1.61434600 \\
\hline $\mathrm{N}$ & 3.78500300 & -2.12214100 & 1.33103400 \\
\hline $\mathrm{N}$ & 4.05778600 & -2.25750800 & -1.10294600 \\
\hline $\mathrm{C}$ & 3.71533100 & -0.92630000 & 2.02753300 \\
\hline $\mathrm{C}$ & 4.65829900 & -2.05932300 & 0.18949700 \\
\hline $\mathrm{C}$ & 5.09696100 & -0.55743200 & 0.15649400 \\
\hline $\mathrm{N}$ & 4.46374600 & -0.00033900 & 1.31895600 \\
\hline $\mathrm{O}$ & 3.16674800 & -0.74315200 & 3.08729800 \\
\hline $\mathrm{C}$ & 4.67409900 & 1.33101500 & 1.81712100 \\
\hline $\mathrm{N}$ & 4.62054000 & -0.10629300 & -1.12497700 \\
\hline $\mathrm{C}$ & 4.03584000 & -1.11143300 & -1.87292800 \\
\hline $\mathrm{O}$ & 3.63795300 & -1.01596700 & -3.01241400 \\
\hline $\mathrm{C}$ & 4.83961900 & 1.20049500 & -1.67754400 \\
\hline $\mathrm{N}$ & 3.83947800 & 2.34281300 & 1.22698000 \\
\hline $\mathrm{N}$ & 3.94755500 & 2.23091400 & -1.21836200 \\
\hline $\mathrm{C}$ & 4.11819100 & 3.00523900 & -0.02116200 \\
\hline $\mathrm{C}$ & 2.80079600 & 2.56137700 & -1.91473900 \\
\hline $\mathrm{C}$ & 2.97813600 & 4.06972000 & -0.11796300 \\
\hline $\mathrm{N}$ & 2.21076500 & 3.62091000 & -1.25384900 \\
\hline
\end{tabular}




$\begin{array}{crrr}\mathrm{C} & 2.85800900 & 2.98301000 & 1.96732300 \\ \mathrm{~N} & 2.34005600 & 3.98980400 & 1.16564300 \\ \mathrm{O} & 2.41922300 & 2.05320800 & -2.94703100 \\ \mathrm{O} & 2.55860900 & 2.75480600 & 3.11418800 \\ \mathrm{C} & 1.15000300 & 4.37560100 & -1.86290000 \\ \mathrm{C} & 1.31343500 & 4.87759200 & 1.64272000 \\ \mathrm{H} & -1.49174400 & -5.47177500 & -1.80622700 \\ \mathrm{H} & -1.10909700 & -4.07086000 & -2.86489300 \\ \mathrm{H} & -1.36906200 & -4.71270700 & 2.76961100 \\ \mathrm{H} & -1.64225400 & -5.86398100 & 1.41995700 \\ \mathrm{H} & 4.03421600 & -4.11947900 & 1.82325700 \\ \mathrm{H} & 3.02948900 & -3.13594400 & 2.94062800 \\ \mathrm{H} & 4.29616500 & -4.29942000 & -1.36438300 \\ \mathrm{H} & 3.50415500 & -3.39607900 & -2.69880300 \\ \mathrm{H} & 5.73037500 & 1.59677400 & 1.66808700 \\ \mathrm{H} & 4.43907900 & 1.31842800 & 2.88478200 \\ \mathrm{H} & 5.86893200 & 1.51632700 & -1.46232700 \\ \mathrm{H} & 4.69603800 & 1.10655500 & -2.75663600 \\ \mathrm{H} & 1.29670200 & 4.77795900 & 2.73096900 \\ \mathrm{H} & 1.57521800 & 5.90687900 & 1.36369900 \\ \mathrm{H} & 1.04354500 & 4.01130600 & -2.88834100 \\ \mathrm{H} & 1.43092700 & 5.44034300 & -1.86997700 \\ \mathrm{H} & -4.09822400 & 4.13847600 & 1.77099200 \\ \mathrm{H} & -3.09450900 & 3.16414200 & 2.89755200 \\ \mathrm{H} & -3.57633300 & 3.40756700 & -2.74408300 \\ \mathrm{H} & -4.36717000 & 4.31026200 & -1.40860400 \\ \mathrm{H} & -5.18252700 & -3.42796500 & 0.04219200 \\ \mathrm{H} & -3.41449200 & -5.07688500 & -0.25341200 \\ \mathrm{H} & 2.66774900 & -5.47571000 & 0.17371100 \\ \mathrm{H} & 0.32335000 & -6.05260500 & -0.18493600 \\ \mathrm{H} & 5.49886500 & -2.75670200 & 0.32015200 \\ \mathrm{H} & 6.18642200 & -0.42016800 & 0.22070700 \\ \mathrm{H} & 3.34818700 & 5.09064000 & -0.29276200 \\ \mathrm{H} & -5.92437100 & -1.46098800 & -1.48578600 \\ \mathrm{H} & -4.73218800 & -1.09689300 & -2.77795300 \\ \mathrm{H} & -4.53410600 & -1.30339900 & 2.86710900 \\ \mathrm{H} & -5.79890200 & -1.61162500 & 1.63004000 \\ \mathrm{H} & 5.12563900 & 3.44588200 & -0.00174000 \\ \mathrm{H} & 1.33570800 & 0.03942300 & -2.06520200 \\ \mathrm{Cl} & 0.97120500 & -0.19349400 & -0.84984500\end{array}$

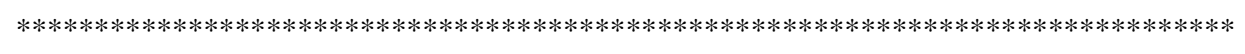

HBr@CB[6]

$\begin{array}{lrrr}\mathrm{C} & -2.10024000 & 4.55837700 & -0.12660300 \\ \mathrm{C} & -3.45488000 & 3.80173000 & 0.02271800 \\ \mathrm{~N} & -3.25646000 & 3.04214000 & 1.23324800 \\ \mathrm{C} & -2.15038900 & 3.46816500 & 1.95288100 \\ \mathrm{~N} & -1.47550200 & 4.37670200 & 1.15129800 \\ \mathrm{~N} & -1.48833000 & 3.89163800 & -1.25257400 \\ \mathrm{H} & -2.22105200 & 5.63154600 & -0.33748500 \\ \mathrm{~N} & -3.55747500 & 3.06342900 & -1.20264800 \\ \mathrm{H} & -4.32040500 & 4.47221100 & 0.12935900 \\ \mathrm{C} & -4.26479400 & 2.22249400 & 1.84519900 \\ \mathrm{O} & -1.86390700 & 3.15958300 & 3.08440200 \\ \mathrm{C} & -4.70475800 & 2.30771600 & -1.63256700 \\ \mathrm{C} & -2.39044700 & 3.10220500 & -1.95013300 \\ \mathrm{O} & -2.21601300 & 2.59588500 & -3.03258800\end{array}$




\begin{tabular}{|c|c|c|c|}
\hline $\mathrm{N}$ & -4.78749300 & 0.95950800 & -1.13981200 \\
\hline C & -5.23651900 & 0.57245100 & 0.16932100 \\
\hline $\mathrm{C}$ & -4.38867700 & -0.11601300 & -1.91791300 \\
\hline C & -5.16982500 & -0.98846500 & 0.12412400 \\
\hline $\mathrm{N}$ & -4.38260900 & 0.90442100 & 1.28172700 \\
\hline $\mathrm{H}$ & -6.25111400 & 0.95948700 & 0.34507500 \\
\hline $\mathrm{N}$ & -4.50624500 & -1.25057800 & -1.12989700 \\
\hline $\mathrm{O}$ & -4.06388700 & -0.07888800 & -3.08018800 \\
\hline $\mathrm{N}$ & -4.45635500 & -1.32247000 & 1.32339500 \\
\hline $\mathrm{H}$ & -6.16054900 & -1.46633100 & 0.13202500 \\
\hline C & -3.98870700 & -0.21088700 & 2.00528300 \\
\hline $\mathrm{O}$ & -3.40525200 & -0.21210000 & 3.06234400 \\
\hline C & -4.34318100 & -2.55866700 & -1.70695800 \\
\hline C & -4.19464300 & -2.65492700 & 1.79189700 \\
\hline $\mathrm{N}$ & -3.05412500 & -3.29869600 & 1.19345200 \\
\hline $\mathrm{N}$ & -3.18791600 & -3.28491600 & -1.25788700 \\
\hline C & -1.95490400 & -3.64424400 & 1.96820400 \\
\hline C & -3.10624300 & -4.04206500 & -0.04343800 \\
\hline C & -1.68864100 & -4.68772400 & -0.12262100 \\
\hline $\mathrm{N}$ & -1.14665500 & -4.45332200 & 1.18487400 \\
\hline C & -1.98140200 & -3.22118200 & -1.93698800 \\
\hline $\mathrm{N}$ & -1.07041800 & -3.98304000 & -1.22001800 \\
\hline C & 0.09689200 & -4.98060600 & 1.67822000 \\
\hline C & 0.16134900 & -4.39795000 & -1.83495900 \\
\hline $\mathrm{O}$ & -1.78211900 & -2.66444900 & -2.98995200 \\
\hline $\mathrm{O}$ & -1.76966700 & -3.35192300 & 3.12491300 \\
\hline $\mathrm{N}$ & 1.28866000 & -4.31465100 & 1.22649100 \\
\hline $\mathrm{N}$ & 1.34843600 & -3.91221300 & -1.18928800 \\
\hline C & 1.94673900 & -3.38037500 & 2.01174800 \\
\hline $\mathrm{C}$ & 1.93997700 & -4.53762100 & -0.03156500 \\
\hline $\mathrm{C}$ & 3.28752300 & -3.77112200 & 0.11976300 \\
\hline $\mathrm{N}$ & 3.06456700 & -2.97086500 & 1.29955500 \\
\hline $\mathrm{O}$ & 1.64067500 & -3.04229400 & 3.12967100 \\
\hline C & 4.08839200 & -2.18573800 & 1.93427800 \\
\hline $\mathrm{N}$ & 3.41077400 & -3.07282100 & -1.12560500 \\
\hline $\mathrm{C}$ & 2.24601600 & -3.11097600 & -1.87523700 \\
\hline $\mathrm{O}$ & 2.07348800 & -2.60128000 & -2.95787800 \\
\hline C & 4.55369500 & -2.30247800 & -1.53641800 \\
\hline $\mathrm{N}$ & 4.27107700 & -0.87310900 & 1.38336900 \\
\hline $\mathrm{N}$ & 4.62029900 & -0.95275900 & -1.04497000 \\
\hline C & 3.73732800 & 0.24163800 & 2.00404600 \\
\hline $\mathrm{C}$ & 5.09922200 & -0.55344400 & 0.25304300 \\
\hline C & 5.03334400 & 1.01103100 & 0.19658400 \\
\hline $\mathrm{N}$ & 4.16386300 & 1.34995700 & 1.29148000 \\
\hline $\mathrm{O}$ & 3.08253300 & 0.25229300 & 3.01883300 \\
\hline C & 3.99225000 & 2.67467700 & 1.82760100 \\
\hline $\mathrm{N}$ & 4.54065100 & 1.26810800 & -1.13116900 \\
\hline C & 4.28706800 & 0.11706300 & -1.85283500 \\
\hline $\mathrm{O}$ & 3.91259300 & 0.06145900 & -3.00214400 \\
\hline C & 4.22354900 & 2.56043300 & -1.66839100 \\
\hline $\mathrm{N}$ & 2.91039900 & 3.42265100 & 1.25574300 \\
\hline $\mathrm{N}$ & 3.01511100 & 3.16659900 & -1.17277800 \\
\hline C & 2.94723400 & 4.04524500 & -0.03997400 \\
\hline C & 1.82747100 & 3.10816200 & -1.87957600 \\
\hline $\mathrm{C}$ & 1.52948700 & 4.68711500 & -0.15946500 \\
\hline $\mathrm{N}$ & 0.93136200 & 3.95344200 & -1.24708500 \\
\hline $\mathrm{C}$ & 1.77335400 & 3.73538700 & 1.97995400 \\
\hline $\mathrm{N}$ & 0.96084500 & 4.48665400 & 1.14337800 \\
\hline $\mathrm{O}$ & 1.63164300 & 2.48678400 & -2.89943800 \\
\hline $\mathrm{O}$ & 1.56083200 & 3.47053700 & 3.13874600 \\
\hline C & -0.28793400 & 4.35051000 & -1.89544300 \\
\hline
\end{tabular}




$\begin{array}{lrrr}\mathrm{C} & -0.28300500 & 5.04390900 & 1.60008800 \\ \mathrm{H} & 0.19300200 & -5.49898900 & -1.87100700 \\ \mathrm{H} & 0.16488000 & -3.99266900 & -2.85052700 \\ \mathrm{H} & 0.06798900 & -4.87603700 & 2.76563100 \\ \mathrm{H} & 0.16582700 & -6.04154700 & 1.40422300 \\ \mathrm{H} & 5.04704600 & -2.72278800 & 1.88481800 \\ \mathrm{H} & 3.78450900 & -2.05915600 & 2.97691100 \\ \mathrm{H} & 5.45899300 & -2.84093800 & -1.22820500 \\ \mathrm{H} & 4.51428000 & -2.23072800 & -2.62586100 \\ \mathrm{H} & 4.93358400 & 3.22686300 & 1.69420500 \\ \mathrm{H} & 3.76642800 & 2.57374300 & 2.89250000 \\ \mathrm{H} & 5.05545900 & 3.24941700 & -1.47225400 \\ \mathrm{H} & 4.10232900 & 2.41783400 & -2.74494300 \\ \mathrm{H} & -0.27219900 & 4.97361500 & 2.69062200 \\ \mathrm{H} & -0.33715700 & 6.09653300 & 1.29152400 \\ \mathrm{H} & -0.28090300 & 3.91167300 & -2.89688500 \\ \mathrm{H} & -0.30940800 & 5.44966300 & -1.96875300 \\ \mathrm{H} & -5.24013000 & 2.72774400 & 1.77636600 \\ \mathrm{H} & -3.98025100 & 2.10274600 & 2.89423900 \\ \mathrm{H} & -4.64990100 & 2.23354200 & -2.72130800 \\ \mathrm{H} & -5.60942800 & 2.85402900 & -1.33820400 \\ \mathrm{H} & -3.91988700 & -4.78140600 & -0.00667700 \\ \mathrm{H} & -1.71365600 & -5.76781300 & -0.33170800 \\ \mathrm{H} & 4.15374400 & -4.43327200 & 0.26634600 \\ \mathrm{H} & 2.06747500 & -5.61682500 & -0.20384500 \\ \mathrm{H} & 6.11572800 & -0.94214700 & 0.41028000 \\ \mathrm{H} & 6.01269200 & 1.49177800 & 0.33320300 \\ \mathrm{H} & 1.55484400 & 5.76103900 & -0.39785000 \\ \mathrm{H} & -5.23536500 & -3.16565100 & -1.50064200 \\ \mathrm{H} & -4.23215200 & -2.41010000 & -2.78401200 \\ \mathrm{H} & -3.98805400 & -2.58480000 & 2.86294200 \\ \mathrm{H} & -5.09278400 & -3.26536200 & 1.62179800 \\ \mathrm{H} & 3.76461900 & 4.77984100 & -0.08745600 \\ \mathrm{H} & 1.29159500 & 0.00699200 & -1.90858100 \\ \mathrm{Br} & 1.18875900 & -0.10806300 & -0.49979300 \\ & & & \\ & & \end{array}$

\begin{tabular}{|c|c|c|c|}
\hline \multicolumn{4}{|c|}{$\mathrm{C}_{2} \mathrm{H}_{6} @\left(\mathrm{CH}_{3}\right)_{12} \mathrm{CB}[6]$} \\
\hline $\mathrm{C}$ & -4.47317300 & -2.40493000 & 0.39119700 \\
\hline $\mathrm{C}$ & -5.05364600 & -0.93696700 & 0.23041600 \\
\hline $\mathrm{N}$ & -4.52889200 & -0.54860600 & -1.07225500 \\
\hline $\mathrm{C}$ & -4.13515000 & -1.64683300 & -1.81867100 \\
\hline $\mathrm{N}$ & -4.09792100 & -2.73750900 & -0.96373800 \\
\hline $\mathrm{N}$ & -3.35268900 & -2.19205700 & 1.30124200 \\
\hline $\mathrm{N}$ & -4.45963700 & -0.24730600 & 1.34686900 \\
\hline $\mathrm{C}$ & -4.83814800 & 0.68070200 & -1.76131500 \\
\hline $\mathrm{O}$ & -3.93229000 & -1.66895600 & -3.01044900 \\
\hline $\mathrm{C}$ & -4.74128200 & 1.09443500 & 1.77966800 \\
\hline $\mathrm{C}$ & -3.42465700 & -0.95652400 & 1.92857400 \\
\hline $\mathrm{O}$ & -2.75285000 & -0.58605500 & 2.86351400 \\
\hline $\mathrm{N}$ & -4.02177400 & 2.13451600 & 1.08446800 \\
\hline $\mathrm{C}$ & -4.38221100 & 2.68706100 & -0.21520400 \\
\hline C & -3.25201100 & 3.01570400 & 1.82491400 \\
\hline $\mathrm{C}$ & -3.30826100 & 3.84256200 & -0.38480100 \\
\hline $\mathrm{N}$ & -4.08864500 & 1.83083300 & -1.33556800 \\
\hline $\mathrm{N}$ & -2.83457100 & 4.02357300 & 0.96844700 \\
\hline $\mathrm{O}$ & -3.04086900 & 2.95896300 & 3.01413900 \\
\hline
\end{tabular}




\begin{tabular}{|c|c|c|c|}
\hline $\mathrm{N}$ & -2.34195600 & 3.22951900 & -1.29092400 \\
\hline $\mathrm{C}$ & -2.85901700 & 2.09987000 & -1.90877100 \\
\hline $\mathrm{O}$ & -2.36236100 & 1.49452500 & -2.83019400 \\
\hline $\mathrm{C}$ & -1.86997400 & 4.99548300 & 1.42075200 \\
\hline C & -1.29260200 & 3.93975100 & -1.98045200 \\
\hline $\mathrm{N}$ & -0.06899500 & 4.09365900 & -1.23840300 \\
\hline $\mathrm{N}$ & -0.49088200 & 4.75833900 & 1.07070700 \\
\hline C & 1.11940700 & 3.74762800 & -1.86083400 \\
\hline $\mathrm{C}$ & 0.15235000 & 5.07422300 & -0.17995600 \\
\hline C & 1.66836100 & 4.80953900 & 0.15118000 \\
\hline $\mathrm{N}$ & 2.15412900 & 4.25332400 & -1.08597100 \\
\hline C & 0.31489600 & 3.96163500 & 1.87406900 \\
\hline $\mathrm{N}$ & 1.54386700 & 3.84828700 & 1.24256700 \\
\hline C & 3.52666600 & 3.98741300 & -1.43812000 \\
\hline C & 2.64628800 & 3.28788000 & 1.98088800 \\
\hline $\mathrm{O}$ & 0.01515900 & 3.50493700 & 2.95209700 \\
\hline $\mathrm{O}$ & 1.23768000 & 3.16934300 & -2.91582800 \\
\hline $\mathrm{N}$ & 4.08218100 & 2.74203400 & -0.97417600 \\
\hline $\mathrm{N}$ & 3.35858100 & 2.22890200 & 1.30829400 \\
\hline C & 4.11634700 & 1.64676800 & -1.82292600 \\
\hline C & 4.46803100 & 2.42215600 & 0.38054500 \\
\hline C & 5.03779500 & 0.94750100 & 0.22801600 \\
\hline $\mathrm{N}$ & 4.51444900 & 0.55380200 & -1.07388300 \\
\hline $\mathrm{O}$ & 3.90885600 & 1.66265900 & -3.01425400 \\
\hline C & 4.81304900 & -0.67894700 & -1.76129100 \\
\hline $\mathrm{N}$ & 4.43833900 & 0.26869500 & 1.34795500 \\
\hline C & 3.42169800 & 0.99600700 & 1.94094900 \\
\hline $\mathrm{O}$ & 2.75707100 & 0.63955700 & 2.88588900 \\
\hline C & 4.71054900 & -1.07265800 & 1.78575100 \\
\hline $\mathrm{N}$ & 4.05928000 & -1.82467300 & -1.33160100 \\
\hline $\mathrm{N}$ & 3.98781900 & -2.11143500 & 1.08995600 \\
\hline $\mathrm{C}$ & 2.84116300 & -2.11390400 & -1.91936800 \\
\hline C & 4.35378100 & -2.67147200 & -0.20566800 \\
\hline $\mathrm{C}$ & 3.28797700 & -3.83349400 & -0.37115700 \\
\hline $\mathrm{N}$ & 2.31616700 & -3.23107600 & -1.28140400 \\
\hline $\mathrm{O}$ & 2.35913500 & -1.53600900 & -2.86592200 \\
\hline C & 1.27979900 & -3.95995900 & -1.97566800 \\
\hline $\mathrm{N}$ & 2.82135100 & -4.01390400 & 0.98333500 \\
\hline C & 3.23275700 & -3.00063800 & 1.83653200 \\
\hline $\mathrm{O}$ & 3.02999700 & -2.94593900 & 3.02714000 \\
\hline C & 1.85514600 & -4.98316300 & 1.43384400 \\
\hline $\mathrm{N}$ & 0.05026700 & -4.12175100 & -1.24506400 \\
\hline $\mathrm{N}$ & 0.47988200 & -4.74018000 & 1.07604800 \\
\hline C & -0.16983300 & -5.07824100 & -0.16486800 \\
\hline C & -0.31604100 & -3.92025700 & 1.86432600 \\
\hline $\mathrm{C}$ & -1.68389300 & -4.79965700 & 0.16850800 \\
\hline $\mathrm{N}$ & -1.54963200 & -3.82243200 & 1.24361200 \\
\hline $\mathrm{C}$ & -1.13956800 & -3.78603500 & -1.87178400 \\
\hline $\mathrm{N}$ & -2.17371800 & -4.26053200 & -1.07571000 \\
\hline $\mathrm{O}$ & -0.00454100 & -3.43563500 & 2.92707800 \\
\hline $\mathrm{O}$ & -1.25921900 & -3.23552500 & -2.94090500 \\
\hline $\mathrm{C}$ & -2.63957200 & -3.24394900 & 1.98358900 \\
\hline C & -3.54735100 & -3.98871100 & -1.42173100 \\
\hline $\mathrm{H}$ & 3.34441200 & 4.07515600 & 2.29099000 \\
\hline $\mathrm{H}$ & 2.20595900 & 2.84389400 & 2.87772300 \\
\hline $\mathrm{H}$ & 3.56698600 & 3.93342500 & -2.52825200 \\
\hline $\mathrm{H}$ & 4.14422100 & 4.82149800 & -1.09477300 \\
\hline $\mathrm{H}$ & 5.88008700 & -0.91551100 & -1.69924100 \\
\hline $\mathrm{H}$ & 4.55226500 & -0.49674400 & -2.80639400 \\
\hline $\mathrm{H}$ & 5.79059400 & -1.24656500 & 1.74307600 \\
\hline $\mathrm{H}$ & 4.38286700 & -1.14317100 & 2.82565100 \\
\hline
\end{tabular}




\begin{tabular}{|c|c|c|c|}
\hline & 1.66088000 & -4.93204600 & -2.31353500 \\
\hline & 1.02301400 & -3.36685100 & -2.85770500 \\
\hline $\mathrm{H}$ & 2.13735100 & -5.97941900 & 1.08321900 \\
\hline $\mathrm{H}$ & 1.90580900 & -4.95667200 & 2.52450900 \\
\hline & -3.59340100 & -3.94183600 & -2.51198400 \\
\hline & -4.16779100 & -4.81702200 & -1.06951000 \\
\hline & -2.18718400 & -2.78810100 & 2.86830900 \\
\hline & -3.33904500 & -4.02176100 & 2.31417700 \\
\hline & -5.90592200 & 0.91226000 & -1.69533800 \\
\hline & -4.58101200 & 0.49725600 & -2.80710800 \\
\hline & -4.41935300 & 1.17013200 & 2.82101700 \\
\hline & -5.82202100 & 1.26127400 & 1.73035400 \\
\hline & -2.15430100 & 5.99033800 & 1.06757800 \\
\hline & -1.92421700 & 4.97039600 & 2.51136900 \\
\hline & -1.03383300 & 3.33661000 & -2.85495900 \\
\hline & -1.66001100 & 4.91337000 & -2.32913800 \\
\hline & -4.28975000 & 4.96876900 & -1.95435700 \\
\hline & -6.52216000 & 2.32829000 & -0.06364400 \\
\hline & -7.00005200 & -1.44997000 & -0.56160400 \\
\hline & -5.03521900 & -4.40677500 & 1.03639100 \\
\hline & -1.96497300 & -6.48070800 & 1.48828200 \\
\hline & 1.16013500 & -6.62120500 & -0.92854800 \\
\hline & 4.65036600 & -5.51871800 & -0.28693000 \\
\hline & 6.49295300 & -2.30528600 & -0.04931100 \\
\hline & 6.96697600 & 1.20119600 & 1.20330200 \\
\hline & 5.04474900 & 4.42792000 & 0.99809700 \\
\hline & 1.94877600 & 6.51175900 & 1.44376700 \\
\hline & -1.18563300 & 6.60290100 & -0.96069300 \\
\hline & -0.14341400 & 6.49418800 & -0.65638400 \\
\hline & 2.44240600 & 6.05216800 & 0.58391200 \\
\hline & 5.48224500 & 3.43112300 & 0.92242100 \\
\hline & 6.56424900 & 0.85266200 & 0.25100800 \\
\hline $\mathrm{C}$ & 5.80917900 & -3.14167900 & -0.20647500 \\
\hline 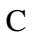 & 3.86648600 & -5.13073800 & -0.93842200 \\
\hline 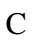 & 0.12068500 & -6.50767000 & -0.61563100 \\
\hline & -2.46062100 & -6.03271900 & 0.62349700 \\
\hline & -5.47632200 & -3.41230200 & 0.95536100 \\
\hline U & -6.58048000 & -0.85192700 & 0.25073400 \\
\hline 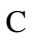 & -5.83564900 & 3.16225100 & -0.22317600 \\
\hline 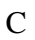 & -3.87929900 & 5.14073300 & -0.95634400 \\
\hline & 0.05052500 & 7.22272900 & 0.13282300 \\
\hline & 0.47648700 & 6.72630000 & -1.52596400 \\
\hline & -4.65877000 & 5.53705900 & -0.30444100 \\
\hline & -3.10955200 & 5.90855500 & -1.04354300 \\
\hline & -6.00060400 & 3.87901400 & 0.58465600 \\
\hline & -6.09317100 & 3.62243600 & -1.17836700 \\
\hline & -6.98209700 & -1.19755900 & 1.20453800 \\
\hline & -6.91795600 & 0.17613800 & 0.10502200 \\
\hline & -5.79492100 & -3.11147500 & 1.95649400 \\
\hline & -6.34724200 & -3.49555300 & 0.30414900 \\
\hline & -3.47589600 & -5.77299200 & 0.92840400 \\
\hline & -2.53149200 & -6.77074200 & -0.17734300 \\
\hline & -0.06536600 & -7.22141800 & 0.18894500 \\
\hline & -0.50735700 & -6.75644700 & -1.47466100 \\
\hline & 3.10145900 & -5.90415400 & -1.01870100 \\
\hline & 4.27274900 & -4.96183300 & -1.93874100 \\
\hline & 5.97381400 & -3.85364300 & 0.60569300 \\
\hline & 6.07097800 & -3.60642700 & -1.15829300 \\
\hline & 6.89454300 & -0.17854900 & 0.11164100 \\
\hline & 6.98917600 & 1.44262400 & -0.56439900 \\
\hline & 5.81132100 & 3.14010500 & 1.92297700 \\
\hline
\end{tabular}




$\begin{array}{lrrr}\mathrm{H} & 6.34573800 & 3.50507200 & 0.26017600 \\ \mathrm{H} & 3.45969900 & 5.80011200 & 0.88881600 \\ \mathrm{H} & 2.50770000 & 6.77816400 & -0.22830200 \\ \mathrm{C} & 0.83054200 & 0.31628900 & -0.30557400 \\ \mathrm{H} & 0.90804200 & 0.45988100 & -1.38696500 \\ \mathrm{C} & -0.33744000 & -0.59799700 & 0.05771700 \\ \mathrm{H} & -1.28135800 & -0.21614400 & -0.34634700 \\ \mathrm{H} & -0.18612900 & -1.59939600 & -0.35647800 \\ \mathrm{H} & 1.77695700 & -0.11409000 & 0.04153500 \\ \mathrm{H} & -0.44730400 & -0.69513700 & 1.14220300 \\ \mathrm{H} & 0.72892700 & 1.30003900 & 0.16497500\end{array}$

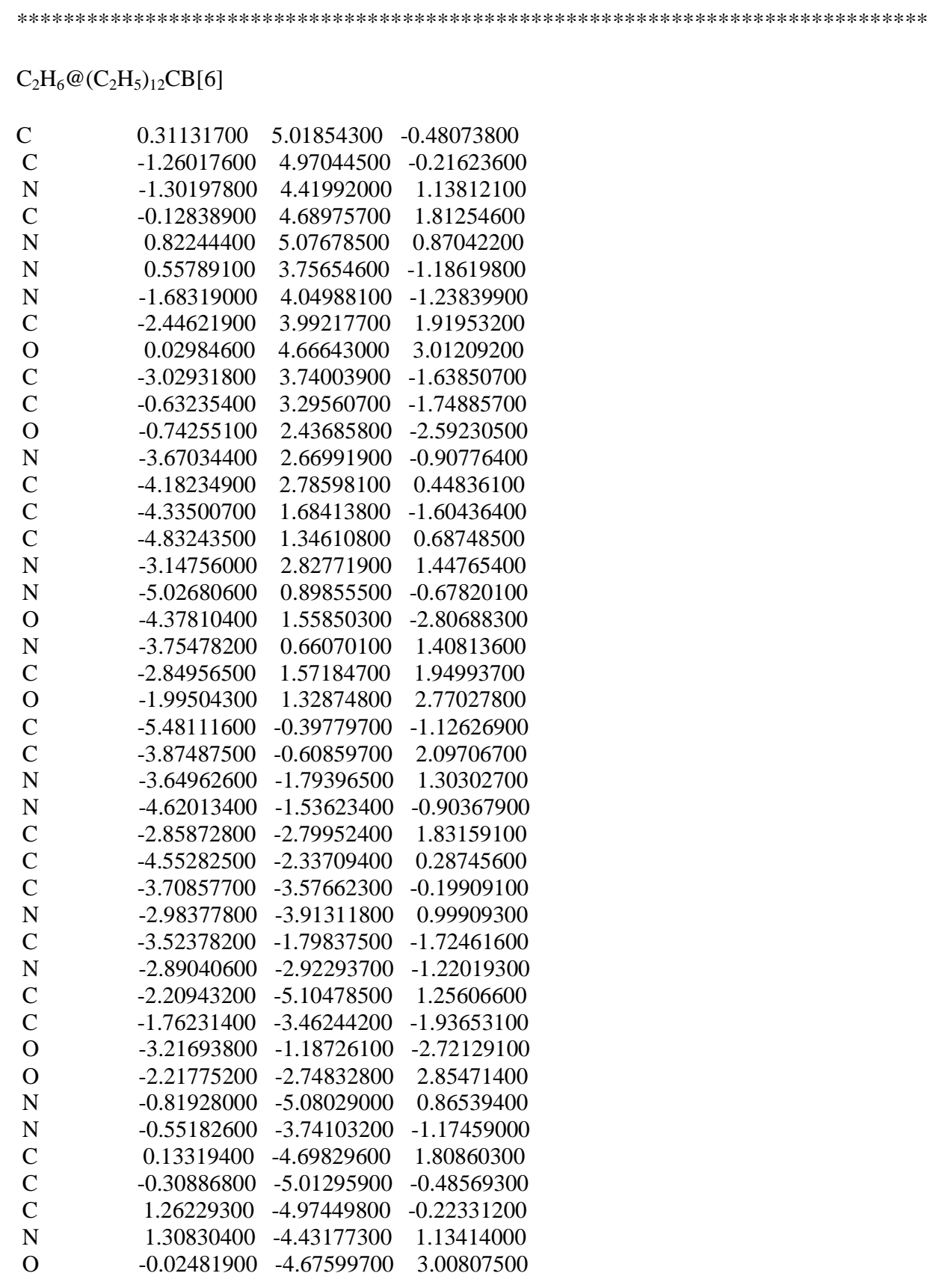




\begin{tabular}{|c|c|c|c|}
\hline C & 2.45798100 & -4.01144600 & 1.91204900 \\
\hline $\mathrm{N}$ & 1.68604300 & -4.04882700 & -1.24005000 \\
\hline $\mathrm{C}$ & 0.64011700 & -3.27102900 & -1.72425400 \\
\hline $\mathrm{O}$ & 0.75759300 & -2.38702100 & -2.54063900 \\
\hline C & 3.03109800 & -3.74245000 & -1.64483400 \\
\hline $\mathrm{N}$ & 3.15867900 & -2.84428700 & 1.44358900 \\
\hline $\mathrm{N}$ & 3.67334100 & -2.67433200 & -0.91443800 \\
\hline C & 2.86933300 & -1.59395900 & 1.96313600 \\
\hline C & 4.18907600 & -2.79467600 & 0.43905200 \\
\hline C & 4.83536800 & -1.35335700 & 0.68033700 \\
\hline $\mathrm{N}$ & 3.75734000 & -0.67489200 & 1.40759900 \\
\hline $\mathrm{O}$ & 2.03378900 & -1.36237100 & 2.80662800 \\
\hline C & 3.87373700 & 0.59754900 & 2.09140200 \\
\hline $\mathrm{N}$ & 5.02356700 & -0.89933800 & -0.68421500 \\
\hline C & 4.33310100 & -1.68498900 & -1.61049600 \\
\hline $\mathrm{O}$ & 4.37263700 & -1.55687000 & -2.81289900 \\
\hline C & 5.47863500 & 0.39686500 & -1.13312300 \\
\hline $\mathrm{N}$ & 3.64306100 & 1.77934900 & 1.29350900 \\
\hline $\mathrm{N}$ & 4.61971200 & 1.53699300 & -0.91166500 \\
\hline $\mathrm{C}$ & 4.54979600 & 2.32979100 & 0.28440200 \\
\hline C & 3.52604600 & 1.80600100 & -1.73539500 \\
\hline C & 3.70932200 & 3.57381800 & -0.19657500 \\
\hline $\mathrm{N}$ & 2.89405300 & 2.93089000 & -1.22670800 \\
\hline C & 2.84452900 & 2.77964800 & 1.82074800 \\
\hline $\mathrm{N}$ & 2.97992100 & 3.90106800 & 1.00062500 \\
\hline $\mathrm{O}$ & 3.21980100 & 1.20062700 & -2.73502900 \\
\hline $\mathrm{O}$ & 2.19224100 & 2.71897500 & 2.83621400 \\
\hline $\mathrm{C}$ & 1.77290000 & 3.48458300 & -1.94482600 \\
\hline C & 2.21155900 & 5.09474200 & 1.26318100 \\
\hline $\mathrm{H}$ & -2.06681600 & -4.35941600 & -2.47872000 \\
\hline $\mathrm{H}$ & -1.49111400 & -2.69757900 & -2.66881300 \\
\hline $\mathrm{H}$ & -2.19415100 & -5.25473900 & 2.33744400 \\
\hline $\mathrm{H}$ & -2.71544600 & -5.94883100 & 0.78499800 \\
\hline $\mathrm{H}$ & 3.17732600 & -4.82190300 & 2.03981800 \\
\hline $\mathrm{H}$ & 2.04585100 & -3.76419200 & 2.89283800 \\
\hline $\mathrm{H}$ & 3.61015400 & -4.66866500 & -1.59750000 \\
\hline $\mathrm{H}$ & 3.00123500 & -3.39669300 & -2.68018700 \\
\hline $\mathrm{H}$ & 4.83931000 & 0.65522300 & 2.60242100 \\
\hline $\mathrm{H}$ & 3.08977500 & 0.60789000 & 2.85293700 \\
\hline $\mathrm{H}$ & 6.44722600 & 0.61171900 & -0.68655500 \\
\hline $\mathrm{H}$ & 5.59627100 & 0.29088900 & -2.21348400 \\
\hline $\mathrm{H}$ & 2.19487700 & 5.23982200 & 2.34519000 \\
\hline $\mathrm{H}$ & 2.72219200 & 5.93875300 & 0.79741900 \\
\hline $\mathrm{H}$ & 1.50283600 & 2.72930200 & -2.68775700 \\
\hline $\mathrm{H}$ & 2.08416100 & 4.38701400 & -2.47379900 \\
\hline $\mathrm{H}$ & -3.16658300 & 4.80013500 & 2.05794300 \\
\hline $\mathrm{H}$ & -2.02709800 & 3.73956700 & 2.89594400 \\
\hline $\mathrm{H}$ & -3.00023100 & 3.39492500 & -2.67413000 \\
\hline $\mathrm{H}$ & -3.61077000 & 4.66477900 & -1.58941200 \\
\hline $\mathrm{H}$ & -6.44925800 & -0.61376500 & -0.67946000 \\
\hline $\mathrm{H}$ & -5.59900500 & -0.29247000 & -2.20669600 \\
\hline $\mathrm{H}$ & -3.08989700 & -0.62008800 & 2.85758700 \\
\hline $\mathrm{H}$ & -4.84038400 & -0.66000900 & 2.60883600 \\
\hline $\mathrm{H}$ & -5.80374300 & 1.95955400 & 2.47507300 \\
\hline $\mathrm{H}$ & -4.52780900 & 4.88384200 & 0.53206700 \\
\hline $\mathrm{H}$ & -1.15342500 & 6.99027600 & 0.36990000 \\
\hline $\mathrm{H}$ & 1.88196200 & 6.19821600 & -1.30805000 \\
\hline $\mathrm{H}$ & 5.36869500 & 4.09758700 & -1.36785500 \\
\hline $\mathrm{H}$ & 6.15522700 & 1.77425600 & 1.53446400 \\
\hline $\mathrm{H}$ & 6.72387800 & -2.28028500 & 0.99633800 \\
\hline $\mathrm{H}$ & 4.53978000 & -4.89258500 & 0.51459300 \\
\hline
\end{tabular}




\begin{tabular}{|c|c|c|c|}
\hline $\mathrm{H}$ & 1.66332600 & -6.78848500 & -1.31160600 \\
\hline $\mathrm{H}$ & -1.88296700 & -6.18077000 & -1.32380000 \\
\hline $\mathrm{H}$ & -5.36405900 & -4.09068600 & -1.37963600 \\
\hline $\mathrm{H}$ & -6.16188200 & -1.78959000 & 1.53632900 \\
\hline C & -5.91073500 & -2.66098300 & 0.94118000 \\
\hline C & -4.67474700 & -4.65240600 & -0.74468500 \\
\hline C & -0.79722300 & -6.20612900 & -1.32673100 \\
\hline C & 1.82040200 & -6.42291100 & -0.29722100 \\
\hline $\mathrm{C}$ & 5.14750000 & -3.99415300 & 0.53748800 \\
\hline C & 6.13720400 & -1.52567900 & 1.51429300 \\
\hline C & 5.90608300 & 2.64892400 & 0.94366500 \\
\hline C & 4.67811000 & 4.65371100 & -0.72944400 \\
\hline C & 0.79591500 & 6.22097400 & -1.31151900 \\
\hline C & -1.82254100 & 6.41811800 & -0.28048100 \\
\hline C & -5.13739700 & 3.98695400 & 0.55471000 \\
\hline $\mathrm{C}$ & -6.13101900 & 1.51818700 & 1.52648700 \\
\hline $\mathrm{H}$ & -5.71533100 & -3.45116600 & 1.67366100 \\
\hline $\mathrm{H}$ & -6.71509500 & 2.28049600 & 1.01709500 \\
\hline $\mathrm{H}$ & -5.60652400 & 3.99243800 & 1.54053800 \\
\hline $\mathrm{H}$ & -1.66717300 & 6.79015200 & -1.29278100 \\
\hline $\mathrm{H}$ & 0.53624600 & 7.13799200 & -0.78096300 \\
\hline $\mathrm{H}$ & 5.25179100 & 5.01162200 & 0.12968300 \\
\hline $\mathrm{H}$ & 5.70927700 & 3.43507800 & 1.68009700 \\
\hline $\mathrm{H}$ & 5.81474600 & -1.97715700 & 2.45972900 \\
\hline $\mathrm{H}$ & 5.62057100 & -4.00183200 & 1.52137900 \\
\hline $\mathrm{H}$ & 1.15074500 & -6.99751100 & 0.35047800 \\
\hline $\mathrm{H}$ & -0.54000900 & -7.12759700 & -0.80263000 \\
\hline $\mathrm{H}$ & -5.25055000 & -5.01909400 & 0.10931300 \\
\hline C & 7.13007100 & 3.00390500 & 0.08401100 \\
\hline $\mathrm{H}$ & 7.29125900 & 4.08072200 & 0.01339600 \\
\hline $\mathrm{H}$ & 8.03337800 & 2.57103600 & 0.52372600 \\
\hline $\mathrm{H}$ & 7.04628400 & 2.62547200 & -0.93748000 \\
\hline C & 4.24912000 & 5.87380300 & -1.54315600 \\
\hline C & 0.32505200 & 6.28763200 & -2.76939400 \\
\hline C & -3.24058700 & 6.82083200 & 0.12315300 \\
\hline $\mathrm{C}$ & -6.17961000 & 4.11143800 & -0.56050600 \\
\hline C & -7.13914200 & 0.41164900 & 1.84826000 \\
\hline $\mathrm{C}$ & -7.13215700 & -3.01244900 & 0.07647100 \\
\hline C & -4.24128300 & -5.86347200 & -1.56911900 \\
\hline C & -0.32512100 & -6.26430100 & -2.78464700 \\
\hline C & 3.23840300 & -6.82994600 & 0.10251400 \\
\hline C & 6.18566700 & -4.11286800 & -0.58211500 \\
\hline C & 7.14046400 & -0.41742900 & 1.84469900 \\
\hline $\mathrm{H}$ & 6.75770200 & -3.19527100 & -0.73781300 \\
\hline $\mathrm{H}$ & 6.89023200 & -4.91483300 & -0.34766000 \\
\hline $\mathrm{H}$ & 5.72044800 & -4.34943700 & -1.54215000 \\
\hline $\mathrm{H}$ & 3.48026700 & -6.58741800 & 1.13819900 \\
\hline $\mathrm{H}$ & 3.30254000 & -7.91860800 & 0.01560900 \\
\hline $\mathrm{H}$ & 4.00983500 & -6.42516500 & -0.55517600 \\
\hline $\mathrm{H}$ & -0.80817100 & -5.51118100 & -3.41022700 \\
\hline $\mathrm{H}$ & 0.74908600 & -6.10574100 & -2.89443500 \\
\hline $\mathrm{H}$ & -0.56541400 & -7.24289500 & -3.20806000 \\
\hline $\mathrm{H}$ & -3.64787100 & -5.59175400 & -2.44409900 \\
\hline $\mathrm{H}$ & -3.70243800 & -6.61435900 & -0.98890500 \\
\hline $\mathrm{H}$ & -5.14529500 & -6.35261800 & -1.94320300 \\
\hline $\mathrm{H}$ & -8.03714700 & -2.58429300 & 0.51728400 \\
\hline $\mathrm{H}$ & -7.04712500 & -2.62686500 & -0.94221100 \\
\hline $\mathrm{H}$ & -7.29102000 & -4.08907200 & -0.00189800 \\
\hline $\mathrm{H}$ & -8.01955200 & 0.89003900 & 2.28745300 \\
\hline $\mathrm{H}$ & -7.48608800 & -0.12374300 & 0.96269400 \\
\hline $\mathrm{H}$ & -6.78602500 & -0.31011100 & 2.58443500 \\
\hline
\end{tabular}




$\begin{array}{crrr}\mathrm{H} & -6.88211300 & 4.91350700 & -0.32030500 \\ \mathrm{H} & -5.71740200 & 4.35114000 & -1.52121800 \\ \mathrm{H} & -6.75337500 & 3.19520200 & -0.71775900 \\ \mathrm{H} & -3.30594600 & 7.90995600 & 0.04298500 \\ \mathrm{H} & -4.01218900 & 6.41922200 & -0.53627200 \\ \mathrm{H} & -3.48085200 & 6.57184100 & 1.15769900 \\ \mathrm{H} & 0.56458200 & 7.26896400 & -3.18684500 \\ \mathrm{H} & 0.80932500 & 5.53851300 & -3.39887000 \\ \mathrm{H} & -0.74897500 & 6.12863800 & -2.88094500 \\ \mathrm{H} & 3.70414200 & 6.61717500 & -0.95908900 \\ \mathrm{H} & 5.15562300 & 6.36860500 & -1.90357500 \\ \mathrm{H} & 3.66380200 & 5.61214100 & -2.42655500 \\ \mathrm{H} & 6.78419000 & 0.29723300 & 2.58628700 \\ \mathrm{H} & 8.02262900 & -0.89569500 & 2.28050800 \\ \mathrm{H} & 7.48558100 & 0.12593800 & 0.96327000 \\ \mathrm{C} & -0.75584300 & -0.11736400 & -0.47367000 \\ \mathrm{H} & -0.77161400 & -0.24763500 & -1.55818400 \\ \mathrm{C} & 0.64584800 & 0.21652600 & 0.02529900 \\ \mathrm{H} & 1.36312800 & -0.54990800 & -0.28823000 \\ \mathrm{H} & 0.99397700 & 1.16794000 & -0.38692000 \\ \mathrm{H} & -1.46687100 & 0.67926300 & -0.23290500 \\ \mathrm{H} & 0.67567200 & 0.29173600 & 1.11620600 \\ \mathrm{H} & -1.12749800 & -1.03737300 & -0.00985000\end{array}$

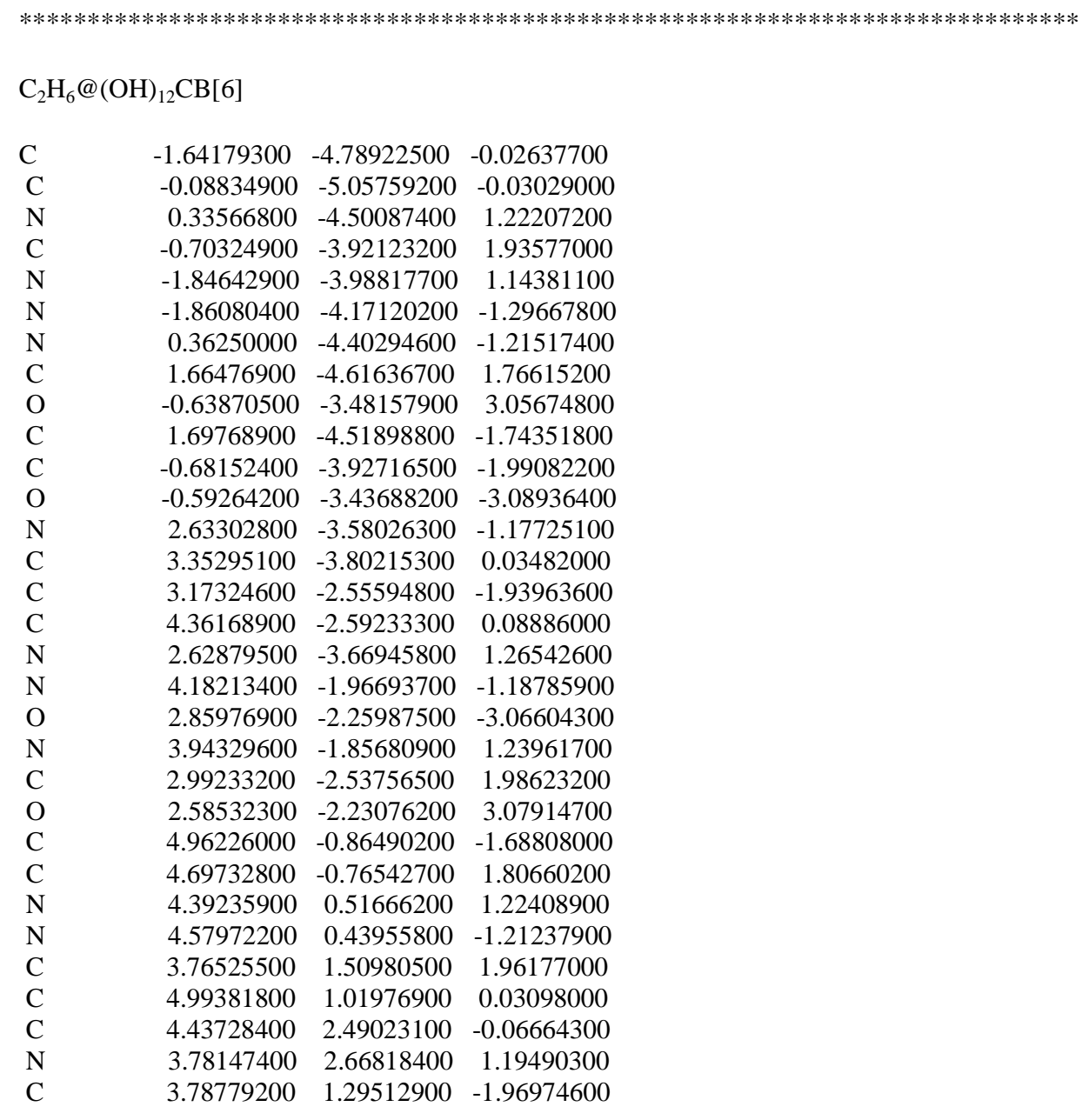




\begin{tabular}{|c|c|c|c|}
\hline $\mathrm{N}$ & 3.61523600 & 2.45923700 & -1.23466300 \\
\hline $\mathrm{C}$ & 3.21888900 & 3.90563500 & 1.66954400 \\
\hline $\mathrm{C}$ & 3.01415800 & 3.62910400 & -1.82533100 \\
\hline $\mathrm{O}$ & 3.36617700 & 1.07787600 & -3.07832700 \\
\hline $\mathrm{O}$ & 3.32573200 & 1.40231700 & 3.07968500 \\
\hline $\mathrm{N}$ & 1.90052800 & 4.22193000 & 1.18409600 \\
\hline $\mathrm{N}$ & 1.74294500 & 3.98467200 & -1.24331500 \\
\hline C & 0.76100200 & 3.97279600 & 1.93803000 \\
\hline $\mathrm{C}$ & 1.60661900 & 4.81339400 & -0.08728300 \\
\hline C & 0.05500400 & 5.07115900 & -0.00328600 \\
\hline $\mathrm{N}$ & -0.33231600 & 4.37670500 & 1.18391700 \\
\hline $\mathrm{O}$ & 0.73302300 & 3.52978800 & 3.05934800 \\
\hline C & -1.64141000 & 4.47960600 & 1.78019700 \\
\hline $\mathrm{N}$ & -0.42303800 & 4.55501500 & -1.25192200 \\
\hline C & 0.57294200 & 3.92434600 & -1.98828800 \\
\hline $\mathrm{O}$ & 0.44862500 & 3.45068000 & -3.09035100 \\
\hline C & -1.77809200 & 4.65906800 & -1.73080000 \\
\hline $\mathrm{N}$ & -2.59927100 & 3.55108000 & 1.23390700 \\
\hline $\mathrm{N}$ & -2.70598000 & 3.69377300 & -1.19784000 \\
\hline C & -3.13141400 & 2.52988000 & 2.00473200 \\
\hline C & -3.37680700 & 3.81234200 & 0.06498600 \\
\hline C & -4.40372300 & 2.61844000 & 0.03109900 \\
\hline $\mathrm{N}$ & -4.20765400 & 1.99511200 & 1.30253200 \\
\hline $\mathrm{O}$ & -2.76493900 & 2.19274300 & 3.10276200 \\
\hline C & -4.89506500 & 0.81568300 & 1.75815800 \\
\hline $\mathrm{N}$ & -4.02807200 & 1.88331400 & -1.14016300 \\
\hline C & -3.12494700 & 2.59292500 & -1.92941300 \\
\hline $\mathrm{O}$ & -2.80178100 & 2.32213700 & -3.05909200 \\
\hline C & -4.85925500 & 0.86104900 & -1.73609700 \\
\hline $\mathrm{N}$ & -4.43593000 & -0.42207400 & 1.16855900 \\
\hline $\mathrm{N}$ & -4.59596900 & -0.47518200 & -1.27015600 \\
\hline $\mathrm{C}$ & -5.02072400 & -0.99393800 & -0.00741800 \\
\hline C & -3.77785200 & -1.35161000 & -1.96988500 \\
\hline $\mathrm{C}$ & -4.45854800 & -2.46473800 & -0.02764400 \\
\hline $\mathrm{N}$ & -3.61557700 & -2.49128600 & -1.18629300 \\
\hline $\mathrm{C}$ & -3.81943900 & -1.38664000 & 1.96104200 \\
\hline $\mathrm{N}$ & -3.82268000 & -2.57800300 & 1.24860400 \\
\hline $\mathrm{O}$ & -3.33028400 & -1.17656600 & -3.07530700 \\
\hline $\mathrm{O}$ & -3.40174300 & -1.22909500 & 3.08080400 \\
\hline C & -3.13532300 & -3.71735800 & -1.78460000 \\
\hline C & -3.14823200 & -3.75564200 & 1.72824100 \\
\hline $\mathrm{H}$ & 3.70253200 & 4.47324200 & -1.73839200 \\
\hline $\mathrm{H}$ & 2.82975300 & 3.39612600 & -2.87600700 \\
\hline $\mathrm{H}$ & 3.14151300 & 3.82044800 & 2.75555300 \\
\hline $\mathrm{H}$ & 3.89346400 & 4.72026100 & 1.39203800 \\
\hline $\mathrm{H}$ & -2.01739400 & 5.49864600 & 1.66091800 \\
\hline $\mathrm{H}$ & -1.52738500 & 4.23672500 & 2.83855700 \\
\hline $\mathrm{H}$ & -2.15152200 & 5.65978700 & -1.49725300 \\
\hline $\mathrm{H}$ & -1.74370400 & 4.50182800 & -2.81102300 \\
\hline $\mathrm{H}$ & -5.96331100 & 0.92734800 & 1.55793300 \\
\hline $\mathrm{H}$ & -4.71391700 & 0.74262200 & 2.83240800 \\
\hline $\mathrm{H}$ & -5.90784500 & 1.10142500 & -1.54507700 \\
\hline $\mathrm{H}$ & -4.65481200 & 0.86784800 & -2.80866900 \\
\hline $\mathrm{H}$ & -2.99733600 & -3.61565200 & 2.80050000 \\
\hline $\mathrm{H}$ & -3.77851200 & -4.62787100 & 1.53979900 \\
\hline $\mathrm{H}$ & -3.00890800 & -3.52382200 & -2.85172900 \\
\hline $\mathrm{H}$ & -3.87955600 & -4.50108200 & -1.62353800 \\
\hline $\mathrm{H}$ & 2.03425100 & -5.62536600 & 1.56309200 \\
\hline $\mathrm{H}$ & 1.58058800 & -4.44492600 & 2.84151500 \\
\hline $\mathrm{H}$ & 1.63759000 & -4.30103700 & -2.81164400 \\
\hline $\mathrm{H}$ & 2.06291200 & -5.53567700 & -1.58083100 \\
\hline
\end{tabular}




$\begin{array}{crrr}\mathrm{H} & 6.01032000 & -1.03428600 & -1.42605700 \\ \mathrm{H} & 4.83324600 & -0.85661800 & -2.77256400 \\ \mathrm{H} & 4.42956100 & -0.70425600 & 2.86333300 \\ \mathrm{H} & 5.76475700 & -0.96923400 & 1.69483700 \\ \mathrm{O} & 5.68284900 & -3.00554500 & 0.29208600 \\ \mathrm{O} & 4.00067700 & -5.03851300 & -0.06369000 \\ \mathrm{O} & 0.22346500 & -6.41579900 & -0.13500000 \\ \mathrm{O} & -2.38433900 & -5.96765400 & 0.12210300 \\ \mathrm{O} & -5.46497800 & -3.42058600 & -0.20096800 \\ \mathrm{O} & -6.41173600 & -0.95293400 & 0.13719600 \\ \mathrm{O} & -5.72384900 & 3.05785400 & -0.13961300 \\ \mathrm{O} & -3.99948200 & 5.05337900 & 0.21596000 \\ \mathrm{O} & -0.25537800 & 6.42557900 & 0.15958100 \\ \mathrm{O} & 2.33517300 & 5.98830600 & -0.30131900 \\ \mathrm{O} & 5.45038900 & 3.43411800 & -0.26964500 \\ \mathrm{O} & 6.38345300 & 0.98035000 & 0.19236800 \\ \mathrm{H} & 5.93672500 & -3.52705800 & -0.47862300 \\ \mathrm{H} & 4.49604200 & -5.16433400 & 0.75441700 \\ \mathrm{H} & -0.18395900 & -6.85737800 & 0.61987500 \\ \mathrm{H} & -2.29606800 & -6.46150100 & -0.70133300 \\ \mathrm{H} & -6.01257500 & -3.39952300 & 0.59236700 \\ \mathrm{H} & -6.78884200 & -1.32554400 & -0.66832300 \\ \mathrm{H} & -5.99984700 & 3.44980900 & 0.69707400 \\ \mathrm{H} & -4.62155000 & 5.14524700 & -0.51604900 \\ \mathrm{H} & 0.01524800 & 6.87570700 & -0.64949900 \\ \mathrm{H} & 2.21085500 & 6.54208000 & 0.47874500 \\ \mathrm{H} & 6.00670100 & 3.42369100 & 0.51838000 \\ \mathrm{H} & 6.77066100 & 1.34446200 & -0.61281300 \\ \mathrm{C} & 0.24986300 & -1.02589000 & -0.24756100 \\ \mathrm{H} & 0.35769100 & -1.14194800 & -1.33048000 \\ \mathrm{C} & 0.85368300 & 0.29091200 & 0.23666300 \\ \mathrm{H} & 1.92201400 & 0.33445200 & 0.00156300 \\ \mathrm{H} & 0.37602500 & 1.14956000 & -0.24648500 \\ \mathrm{H} & -0.81765700 & -1.08971700 & -0.00955800 \\ \mathrm{H} & 0.74843400 & 0.40795600 & 1.31933600 \\ \mathrm{H} & 0.74072500 & -1.88069800 & 0.22933800 \\ & & & \\ & & & \\ & & & \end{array}$

$\mathrm{C}_{2} \mathrm{H}_{6} @(\mathrm{CN})_{12} \mathrm{CB}[6]$

$\begin{array}{lrrr}\mathrm{C} & -3.06438200 & 4.05917000 & -0.19811400 \\ \mathrm{C} & -4.20204200 & 2.95020500 & -0.07559500 \\ \mathrm{~N} & -3.86380500 & 2.30025400 & 1.16694400 \\ \mathrm{C} & -2.93617800 & 3.02135200 & 1.91373100 \\ \mathrm{~N} & -2.47290800 & 4.05485500 & 1.10886400 \\ \mathrm{~N} & -2.25163900 & 3.54868400 & -1.27556500 \\ \mathrm{~N} & -4.02714600 & 2.18210400 & -1.27272300 \\ \mathrm{C} & -4.68900000 & 1.30260200 & 1.80966700 \\ \mathrm{O} & -2.62378500 & 2.81197100 & 3.05483000 \\ \mathrm{C} & -4.88375900 & 1.10979500 & -1.71171500 \\ \mathrm{C} & -2.85508900 & 2.49011400 & -1.94838100 \\ \mathrm{O} & -2.45518800 & 1.96378300 & -2.95274300 \\ \mathrm{~N} & -4.59342800 & -0.17182200 & -1.11169700 \\ \mathrm{C} & -5.10000000 & -0.60420100 & 0.16559300 \\ \mathrm{C} & -4.10560200 & -1.21982900 & -1.88685600 \\ \mathrm{C} & -4.64161200 & -2.13149600 & 0.22444300 \\ \mathrm{~N} & -4.47862100 & -0.03769000 & 1.32715900 \\ \mathrm{~N} & -4.10564800 & -2.35633400 & -1.08812300\end{array}$




\begin{tabular}{|c|c|c|c|}
\hline $\mathrm{O}$ & -3.78233600 & -1.16045900 & -3.04235400 \\
\hline $\mathrm{N}$ & -3.70051200 & -2.13644600 & 1.31422400 \\
\hline $\mathrm{C}$ & -3.64114800 & -0.92301000 & 1.99050200 \\
\hline $\mathrm{O}$ & -3.01314600 & -0.69439300 & 2.98984800 \\
\hline C & -3.66008100 & -3.63083200 & -1.59654900 \\
\hline C & -3.14994400 & -3.32992300 & 1.90666800 \\
\hline $\mathrm{N}$ & -1.97139100 & -3.82489700 & 1.23689000 \\
\hline $\mathrm{N}$ & -2.33757200 & -4.02669500 & -1.17546800 \\
\hline C & -0.77052900 & -3.93193200 & 1.93334900 \\
\hline C & -2.00747500 & -4.66591200 & 0.06427900 \\
\hline C & -0.47120900 & -5.03013400 & -0.12235900 \\
\hline $\mathrm{N}$ & 0.09776600 & -4.67734300 & 1.14547800 \\
\hline C & -1.21455900 & -3.70459300 & -1.92466700 \\
\hline $\mathrm{N}$ & -0.10656400 & -4.20353400 & -1.24796400 \\
\hline C & 1.44461000 & -4.96746900 & 1.57699100 \\
\hline C & 1.16902500 & -4.27033600 & -1.91686100 \\
\hline $\mathrm{O}$ & -1.20481400 & -3.13061900 & -2.98166200 \\
\hline $\mathrm{O}$ & -0.54061800 & -3.49221300 & 3.02789400 \\
\hline $\mathrm{N}$ & 2.45124300 & -4.04436600 & 1.11272700 \\
\hline $\mathrm{N}$ & 2.21334900 & -3.51642000 & -1.26467400 \\
\hline C & 2.91153900 & -3.00917300 & 1.91744300 \\
\hline C & 3.03321600 & -4.03903300 & -0.19782700 \\
\hline C & 4.17373500 & -2.93526500 & -0.07386700 \\
\hline $\mathrm{N}$ & 3.83854400 & -2.28730700 & 1.17010600 \\
\hline $\mathrm{O}$ & 2.59932400 & -2.80045900 & 3.05855800 \\
\hline C & 4.66551500 & -1.28923400 & 1.80935700 \\
\hline $\mathrm{N}$ & 3.99966900 & -2.16301700 & -1.26811500 \\
\hline C & 2.81963300 & -2.45785800 & -1.93570000 \\
\hline $\mathrm{O}$ & 2.41451400 & -1.92220500 & -2.93342100 \\
\hline $\mathrm{C}$ & 4.87007500 & -1.10257200 & -1.71063400 \\
\hline $\mathrm{N}$ & 4.45807500 & 0.04966300 & 1.32220200 \\
\hline $\mathrm{N}$ & 4.59771100 & 0.18468400 & -1.11824100 \\
\hline C & 3.62700200 & 0.93804000 & 1.98914000 \\
\hline C & 5.08752700 & 0.61722200 & 0.16444500 \\
\hline C & 4.62202000 & 2.14379900 & 0.21870100 \\
\hline $\mathrm{N}$ & 3.69244700 & 2.15217400 & 1.31727000 \\
\hline $\mathrm{O}$ & 2.99759300 & 0.70988400 & 2.98805400 \\
\hline C & 3.13112100 & 3.34350900 & 1.90211600 \\
\hline $\mathrm{N}$ & 4.07036000 & 2.35855200 & -1.08927900 \\
\hline $\mathrm{C}$ & 4.08267400 & 1.22191800 & -1.88720300 \\
\hline $\mathrm{O}$ & 3.74906200 & 1.15661700 & -3.03968900 \\
\hline C & 3.62540700 & 3.62980000 & -1.60589600 \\
\hline $\mathrm{N}$ & 1.94962400 & 3.82915700 & 1.22892500 \\
\hline $\mathrm{N}$ & 2.30575500 & 4.03239600 & -1.18329100 \\
\hline C & 1.98290000 & 4.67210600 & 0.05763700 \\
\hline $\mathrm{C}$ & 1.17821900 & 3.70388400 & -1.92331800 \\
\hline C & 0.44715300 & 5.04073500 & -0.12346100 \\
\hline $\mathrm{N}$ & 0.07426900 & 4.21478000 & -1.24750900 \\
\hline C & 0.74754000 & 3.92935900 & 1.92428000 \\
\hline $\mathrm{N}$ & -0.11833600 & 4.68750700 & 1.14550300 \\
\hline $\mathrm{O}$ & 1.16254500 & 3.11620500 & -2.97243300 \\
\hline $\mathrm{O}$ & 0.51535500 & 3.47606600 & 3.01290600 \\
\hline C & -1.20028200 & 4.29962100 & -1.91852700 \\
\hline C & -1.46565400 & 4.97596800 & 1.57694300 \\
\hline $\mathrm{H}$ & 1.48003400 & -5.31647200 & -2.03285900 \\
\hline $\mathrm{H}$ & 1.02382300 & -3.82030400 & -2.90227900 \\
\hline $\mathrm{H}$ & 1.44668100 & -4.89988300 & 2.66668500 \\
\hline $\mathrm{H}$ & 1.70959800 & -5.98375900 & 1.26893000 \\
\hline $\mathrm{H}$ & 5.72504600 & -1.54976300 & 1.70468500 \\
\hline $\mathrm{H}$ & 4.38895500 & -1.29439500 & 2.86620800 \\
\hline $\mathrm{H}$ & 5.90909400 & -1.39297900 & -1.52064500 \\
\hline
\end{tabular}




\begin{tabular}{|c|c|c|c|}
\hline $\mathrm{H}$ & 4.71228000 & -0.98306700 & -2.78441700 \\
\hline $\mathrm{H}$ & 3.89400000 & 4.13082800 & 1.93942800 \\
\hline $\mathrm{H}$ & 2.82244600 & 3.08466800 & 2.91802100 \\
\hline $\mathrm{H}$ & 4.34072500 & 4.41225200 & -1.33337700 \\
\hline $\mathrm{H}$ & 3.59119700 & 3.52212600 & -2.69182600 \\
\hline $\mathrm{H}$ & -1.46869700 & 4.90450400 & 2.66636400 \\
\hline $\mathrm{H}$ & -1.72950500 & 5.99361700 & 1.27239600 \\
\hline $\mathrm{H}$ & -1.05894700 & 3.85831200 & -2.90843400 \\
\hline $\mathrm{H}$ & -1.50137600 & 5.34966200 & -2.02397100 \\
\hline $\mathrm{H}$ & -5.74902000 & 1.56005300 & 1.70325900 \\
\hline $\mathrm{H}$ & -4.41332300 & 1.31201500 & 2.86671400 \\
\hline $\mathrm{H}$ & -4.72211300 & 0.98775900 & -2.78477400 \\
\hline $\mathrm{H}$ & -5.92689800 & 1.38763400 & -1.52514700 \\
\hline $\mathrm{H}$ & -4.37312800 & -4.41196100 & -1.31469300 \\
\hline $\mathrm{H}$ & -3.63060900 & -3.53190200 & -2.68345000 \\
\hline $\mathrm{H}$ & -2.84124300 & -3.06984400 & 2.92229900 \\
\hline $\mathrm{H}$ & -3.91868000 & -4.11151300 & 1.94575200 \\
\hline C & 3.53933200 & -5.38757400 & -0.54094600 \\
\hline C & 5.54605100 & -3.48877200 & -0.01198800 \\
\hline C & 6.55549600 & 0.42988600 & 0.23761900 \\
\hline C & 5.72555100 & 3.10021500 & 0.46887300 \\
\hline $\mathrm{C}$ & 2.86298900 & 5.84075500 & 0.28364300 \\
\hline C & 0.20127200 & 6.46952100 & -0.42195400 \\
\hline $\mathrm{C}$ & -3.57493500 & 5.40987100 & -0.52593800 \\
\hline C & -5.57619000 & 3.49880400 & -0.01155500 \\
\hline $\mathrm{C}$ & -6.56809600 & -0.41352100 & 0.22488000 \\
\hline C & -5.74714300 & -3.08095700 & 0.49154500 \\
\hline C & -2.88391700 & -5.83680400 & 0.29343600 \\
\hline C & -0.22168800 & -6.45798100 & -0.42338900 \\
\hline $\mathrm{N}$ & 6.64269000 & -3.84833600 & 0.06390300 \\
\hline $\mathrm{N}$ & 7.68920000 & 0.20414700 & 0.27728700 \\
\hline $\mathrm{N}$ & 6.53421400 & 3.90386800 & 0.66384100 \\
\hline $\mathrm{N}$ & 3.60489100 & 6.70429900 & 0.48720600 \\
\hline $\mathrm{N}$ & -0.06048500 & 7.56641000 & -0.67863800 \\
\hline $\mathrm{N}$ & -3.90164800 & 6.48659200 & -0.79353500 \\
\hline $\mathrm{N}$ & -6.67418700 & 3.85406700 & 0.06512900 \\
\hline $\mathrm{N}$ & -7.70191600 & -0.18656600 & 0.25250700 \\
\hline $\mathrm{N}$ & -6.55752500 & -3.87937500 & 0.70035500 \\
\hline $\mathrm{N}$ & -3.62383400 & -6.70138900 & 0.49986800 \\
\hline $\mathrm{N}$ & 0.04304500 & -7.55349900 & -0.68292100 \\
\hline $\mathrm{N}$ & 3.86444300 & -6.46176600 & -0.82034600 \\
\hline $\mathrm{C}$ & -0.14579000 & -0.65015700 & -0.54666900 \\
\hline $\mathrm{H}$ & -0.00301800 & -1.12826000 & -1.51971800 \\
\hline $\mathrm{C}$ & 1.05222200 & 0.22704600 & -0.18995100 \\
\hline $\mathrm{H}$ & 1.96356500 & -0.37459300 & -0.09461400 \\
\hline $\mathrm{H}$ & 1.23051800 & 0.97109000 & -0.97263900 \\
\hline $\mathrm{H}$ & -1.06425700 & -0.05747800 & -0.60726400 \\
\hline $\mathrm{H}$ & 0.90392200 & 0.74477400 & 0.76292200 \\
\hline $\mathrm{H}$ & -0.30309800 & -1.42868300 & 0.20788300 \\
\hline
\end{tabular}




\begin{tabular}{|c|c|c|c|}
\hline $\mathrm{N}$ & 2.77179700 & 2.94016200 & 1.31257300 \\
\hline $\mathrm{N}$ & 4.24456200 & 1.25829700 & 1.34412300 \\
\hline C & 4.86557200 & 0.40905000 & -1.75391000 \\
\hline $\mathrm{O}$ & 3.38789800 & 2.43589100 & -3.01739100 \\
\hline C & 4.81352900 & 0.01636400 & 1.78937000 \\
\hline C & 3.09968600 & 1.74851800 & 1.94090900 \\
\hline $\mathrm{O}$ & 2.53276000 & 1.26257100 & 2.89301300 \\
\hline $\mathrm{N}$ & 4.36060200 & -1.16423600 & 1.09473200 \\
\hline $\mathrm{C}$ & 4.85763600 & -1.63449700 & -0.19154400 \\
\hline C & 3.78451400 & -2.18274300 & 1.83105900 \\
\hline C & 4.08531400 & -3.01159900 & -0.35970600 \\
\hline $\mathrm{N}$ & 4.39576600 & -0.88149300 & -1.32963500 \\
\hline $\mathrm{N}$ & 3.62262600 & -3.26910000 & 0.98405900 \\
\hline $\mathrm{O}$ & 3.53500500 & -2.16066700 & 3.01422400 \\
\hline $\mathrm{N}$ & 3.03816000 & -2.65816100 & -1.31124500 \\
\hline C & 3.28977700 & -1.44585300 & -1.93486000 \\
\hline $\mathrm{O}$ & 2.69606500 & -0.99447400 & -2.88791400 \\
\hline C & 2.91137000 & -4.43521200 & 1.44161800 \\
\hline $\mathrm{C}$ & 2.16966700 & -3.59278300 & -1.98219300 \\
\hline $\mathrm{N}$ & 0.99591400 & -3.98051600 & -1.23925100 \\
\hline $\mathrm{N}$ & 1.51750200 & -4.52698400 & 1.08270800 \\
\hline C & -0.23552000 & -3.90124500 & -1.87577200 \\
\hline C & 0.97532600 & -4.97977600 & -0.17352800 \\
\hline C & -0.56716300 & -5.04903500 & 0.13925700 \\
\hline $\mathrm{N}$ & -1.14941300 & -4.60146100 & -1.10048900 \\
\hline $\mathrm{C}$ & 0.54805300 & -3.92642600 & 1.87521300 \\
\hline $\mathrm{N}$ & -0.66837200 & -4.09039900 & 1.23462800 \\
\hline $\mathrm{C}$ & -2.54419200 & -4.64146000 & -1.46801900 \\
\hline C & -1.87170700 & -3.79465200 & 1.96795100 \\
\hline $\mathrm{O}$ & 0.72959200 & -3.41025300 & 2.95331500 \\
\hline $\mathrm{O}$ & -0.46192700 & -3.37491600 & -2.93967800 \\
\hline $\mathrm{N}$ & -3.37410400 & -3.56270500 & -0.99317800 \\
\hline $\mathrm{N}$ & -2.79891900 & -2.92007600 & 1.29376500 \\
\hline C & -3.67664900 & -2.50358000 & -1.83485600 \\
\hline C & -3.83214200 & -3.35805700 & 0.36242600 \\
\hline $\mathrm{C}$ & -4.73344900 & -2.05663000 & 0.22561900 \\
\hline $\mathrm{N}$ & -4.33535800 & -1.54890300 & -1.08020700 \\
\hline $\mathrm{O}$ & -3.46643900 & -2.45819800 & -3.02491900 \\
\hline C & -4.92324600 & -0.41513900 & -1.75114700 \\
\hline $\mathrm{N}$ & -4.29469800 & -1.25646900 & 1.34132000 \\
\hline C & -3.13321100 & -1.72987400 & 1.92327000 \\
\hline $\mathrm{O}$ & -2.55801400 & -1.22893800 & 2.86149600 \\
\hline C & -4.86725600 & -0.01654700 & 1.78997800 \\
\hline $\mathrm{N}$ & -4.44642200 & 0.87263800 & -1.32463500 \\
\hline $\mathrm{N}$ & -4.40988800 & 1.16438900 & 1.09855400 \\
\hline $\mathrm{C}$ & -3.32786200 & 1.42472100 & -1.92284100 \\
\hline C & -4.90541700 & 1.63213800 & -0.18980600 \\
\hline $\mathrm{C}$ & -4.12387100 & 3.00264800 & -0.36051600 \\
\hline $\mathrm{N}$ & -3.06347100 & 2.63211700 & -1.29207800 \\
\hline $\mathrm{O}$ & -2.73617400 & 0.96610800 & -2.87210100 \\
\hline C & -2.20392400 & 3.56503600 & -1.97825700 \\
\hline $\mathrm{N}$ & -3.67791000 & 3.27217500 & 0.98724300 \\
\hline C & -3.84403200 & 2.18888500 & 1.83754100 \\
\hline $\mathrm{O}$ & -3.60444200 & 2.17116000 & 3.02260900 \\
\hline C & -2.95478100 & 4.43480300 & 1.43875700 \\
\hline $\mathrm{N}$ & -1.03412300 & 3.97281400 & -1.24352600 \\
\hline $\mathrm{N}$ & -1.56068900 & 4.51801200 & 1.07590900 \\
\hline $\mathrm{C}$ & -1.01927800 & 4.97285500 & -0.18008000 \\
\hline C & -0.58806000 & 3.93156200 & 1.87448000 \\
\hline $\mathrm{C}$ & 0.52266500 & 5.05018500 & 0.13253700 \\
\hline $\mathrm{N}$ & 0.62970700 & 4.09113000 & 1.22905100 \\
\hline
\end{tabular}




\begin{tabular}{|c|c|c|c|}
\hline C & 0.19566100 & 3.89243900 & -1.87677300 \\
\hline $\mathrm{N}$ & 1.10476200 & 4.60666800 & -1.10832500 \\
\hline $\mathrm{O}$ & -0.76566000 & 3.43028400 & 2.95966200 \\
\hline $\mathrm{O}$ & 0.42771500 & 3.35230800 & -2.93323900 \\
\hline C & 1.83584600 & 3.81620800 & 1.97049800 \\
\hline C & 2.50010500 & 4.63700300 & -1.47136800 \\
\hline $\mathrm{H}$ & -2.37724500 & -4.71982800 & 2.27093600 \\
\hline $\mathrm{H}$ & -1.54612200 & -3.26831900 & 2.86922600 \\
\hline $\mathrm{H}$ & -2.58318700 & -4.57540500 & -2.55758700 \\
\hline $\mathrm{H}$ & -2.96309900 & -5.59976400 & -1.14957700 \\
\hline $\mathrm{H}$ & -6.01445100 & -0.43158900 & -1.66804300 \\
\hline $\mathrm{H}$ & -4.64677500 & -0.52765900 & -2.80208900 \\
\hline $\mathrm{H}$ & -5.95817100 & -0.09838400 & 1.75134100 \\
\hline $\mathrm{H}$ & -4.55983000 & 0.12115700 & 2.82929500 \\
\hline $\mathrm{H}$ & -2.77807200 & 4.44079800 & -2.30624100 \\
\hline $\mathrm{H}$ & -1.83127200 & 3.04617400 & -2.86548100 \\
\hline $\mathrm{H}$ & -3.46132900 & 5.34052200 & 1.09508700 \\
\hline $\mathrm{H}$ & -2.98963100 & 4.39314100 & 2.52959700 \\
\hline $\mathrm{H}$ & 2.54166000 & 4.56921800 & -2.56069600 \\
\hline $\mathrm{H}$ & 2.92539800 & 5.59240700 & -1.15291200 \\
\hline $\mathrm{H}$ & 1.51311500 & 3.29949900 & 2.87843900 \\
\hline $\mathrm{H}$ & 2.33206900 & 4.75035600 & 2.26091800 \\
\hline $\mathrm{H}$ & 5.95729000 & 0.42850000 & -1.67758700 \\
\hline $\mathrm{H}$ & 4.58261900 & 0.52280900 & -2.80300000 \\
\hline $\mathrm{H}$ & 4.50156900 & -0.12321500 & 2.82707500 \\
\hline $\mathrm{H}$ & 5.90487200 & 0.09657600 & 1.75578000 \\
\hline $\mathrm{H}$ & 3.42265500 & -5.33886300 & 1.09959600 \\
\hline $\mathrm{H}$ & 2.94839300 & -4.38950900 & 2.53221500 \\
\hline $\mathrm{H}$ & 1.80051900 & -3.08203300 & -2.87565700 \\
\hline $\mathrm{H}$ & 2.73587800 & -4.47787500 & -2.29832000 \\
\hline $\mathrm{H}$ & 5.35829200 & -3.90557400 & -1.86712100 \\
\hline $\mathrm{H}$ & 6.85572300 & -0.79180900 & -0.01379200 \\
\hline $\mathrm{H}$ & 6.48323000 & 2.97263000 & -0.55153100 \\
\hline $\mathrm{H}$ & 3.89833300 & 5.45866500 & 0.92506200 \\
\hline $\mathrm{H}$ & 0.43730400 & 6.77720900 & 1.42019200 \\
\hline $\mathrm{H}$ & -2.67315800 & 6.16950600 & -0.93369800 \\
\hline $\mathrm{H}$ & -5.81398200 & 4.35618200 & -0.23095800 \\
\hline $\mathrm{H}$ & -6.90716800 & 0.79970100 & -0.01055600 \\
\hline $\mathrm{H}$ & -6.53468700 & -2.75423000 & 1.22671200 \\
\hline $\mathrm{H}$ & -3.92764900 & -5.45309300 & 0.94476800 \\
\hline $\mathrm{H}$ & -0.48290500 & -6.77704300 & 1.42421800 \\
\hline $\mathrm{H}$ & 2.62759800 & -6.18497900 & -0.91609500 \\
\hline C & 1.58010000 & -6.30402200 & -0.63452800 \\
\hline C & -1.05624800 & -6.43299900 & 0.55996400 \\
\hline C & -4.58338500 & -4.58322800 & 0.88575800 \\
\hline $\mathrm{C}$ & -6.24005000 & -2.31868400 & 0.27071300 \\
\hline C & -6.42873900 & 1.76814400 & -0.16959600 \\
\hline C & -4.98351400 & 4.14368500 & -0.90540700 \\
\hline C & -1.62861600 & 6.29404000 & -0.64405500 \\
\hline C & 1.00664300 & 6.43617600 & 0.55206000 \\
\hline C & 4.55348300 & 4.58855800 & 0.85964100 \\
\hline $\mathrm{C}$ & 6.19174700 & 2.30679900 & 0.26388300 \\
\hline C & 6.38184500 & -1.76275900 & -0.17042900 \\
\hline C & 4.95481700 & -4.15537800 & -0.88300600 \\
\hline $\mathrm{H}$ & 1.53349400 & -7.05449300 & 0.15622500 \\
\hline $\mathrm{H}$ & 1.04412500 & -6.66771000 & -1.51461200 \\
\hline $\mathrm{H}$ & 5.77402500 & -4.36453700 & -0.19381900 \\
\hline $\mathrm{H}$ & 4.37758400 & -5.07517700 & -0.98924500 \\
\hline $\mathrm{H}$ & 6.69181800 & -2.41219800 & 0.65157000 \\
\hline $\mathrm{H}$ & 6.75724200 & -2.16307900 & -1.11349800 \\
\hline $\mathrm{H}$ & 6.49128900 & 2.74368500 & 1.21774400 \\
\hline
\end{tabular}




$\begin{array}{lrrr}\mathrm{H} & 6.74889900 & 1.37647500 & 0.13828700 \\ \mathrm{H} & 4.95941100 & 4.40633100 & 1.85742800 \\ \mathrm{H} & 5.36399200 & 4.84487100 & 0.17609500 \\ \mathrm{H} & 2.05939800 & 6.41941700 & 0.83888800 \\ \mathrm{H} & 0.89596300 & 7.15539700 & -0.26113800 \\ \mathrm{H} & -1.59110900 & 7.04495200 & 0.14689600 \\ \mathrm{H} & -1.08827300 & 6.66018600 & -1.52040400 \\ \mathrm{H} & -4.40575600 & 5.06349700 & -1.00654700 \\ \mathrm{H} & -5.37125900 & 3.88792300 & -1.89445400 \\ \mathrm{H} & -6.73589900 & 2.42149800 & 0.65039300 \\ \mathrm{H} & -6.80178800 & 2.16750200 & -1.11399000 \\ \mathrm{H} & -6.80149200 & -1.39092500 & 0.14412400 \\ \mathrm{H} & -6.53070500 & -2.98820700 & -0.54197800 \\ \mathrm{H} & -4.97188000 & -4.39371200 & 1.88925200 \\ \mathrm{H} & -5.40602100 & -4.84426200 & 0.21878100 \\ \mathrm{H} & -2.10671400 & -6.41109000 & 0.85438200 \\ \mathrm{H} & -0.95430500 & -7.15260000 & -0.25417600 \\ \mathrm{C} & 0.87351600 & -0.47361100 & 0.40908800 \\ \mathrm{H} & 0.78344800 & -0.37784500 & 1.48730500 \\ \mathrm{H} & 0.99114900 & -1.47931800 & 0.01129600 \\ \mathrm{C} & 0.83821500 & 0.58270500 & -0.39915200 \\ \mathrm{H} & 0.92962700 & 0.48897700 & -1.47768600 \\ \mathrm{H} & 0.70944300 & 1.58642700 & 0.00025200\end{array}$

$\mathrm{C}_{2} \mathrm{H}_{4} @\left(\mathrm{C}_{2} \mathrm{H}_{5}\right)_{12} \mathrm{CB}[6]$

C $\quad \begin{array}{llll}0.39132800 & -4.99973800 & 0.49942900\end{array}$

$\begin{array}{llll}\mathrm{C} & -1.94017700 & -4.75423200 & 0.22674500\end{array}$

$\mathrm{N} \quad \quad-1.90017000 \quad-4.19238700 \quad-1.12363400$

C $\quad-0.76768100 \quad-4.61018900 \quad-1.79415400$

$\begin{array}{llll}\mathrm{N} & 0.11980200 & -5.12003900 & -0.84845700\end{array}$

$\begin{array}{llll}\mathrm{N} & 0.00626600 & -3.77607100 & 1.20191000\end{array}$

$\mathrm{N} \quad-2.25114700 \quad-3.79558500 \quad 1.25462500$

C $\quad-2.97762400 \quad-3.61844700 \quad-1.90704300$

O $\quad-0.60072100 \quad-4.60067800-2.99260100$

$\begin{array}{llll}\text { C } & -3.54873600 & -3.31528300 & 1.64784500\end{array}$

C $\quad-1.11794600 \quad-3.16242200 \quad 1.75176400$

$\begin{array}{llll}\mathrm{O} & -1.12225800 & -2.27490200 & 2.57277600\end{array}$

$\mathrm{N} \quad-4.03376600 \quad-2.16345500 \quad 0.92383800$

$\begin{array}{llll}\text { C } & -4.54359800 & -2.19656300 & -0.43596800\end{array}$

$\begin{array}{llll}\text { C } & -4.54949000 & -1.09568800 & 1.62347600\end{array}$

$\begin{array}{llll}\text { C } & -4.98461000 & -0.67754700 & -0.66759700\end{array}$

$\mathrm{N} \quad-\quad \begin{array}{llll}\mathrm{C} & -3.51891700 & -2.37388900 & -1.43161900\end{array}$

$\begin{array}{llll}\mathrm{N} & -5.11872800 & -0.21388700 & 0.70001200\end{array}$

$\begin{array}{llll}\mathrm{O} & -4.57553700 & -0.96963600 & 2.82666500\end{array}$

N $\quad-3.81980900 \quad-0.14319800-1.38317700$

C $\quad-3.06274400 \quad-1.17077500 \quad-1.94334800$

$\begin{array}{llll}\text { O } & -2.20898100 & -1.05059200 & -2.79154800\end{array}$

$\begin{array}{llll}\text { C } & -5.37161100 & 1.13401500 & 1.15492200\end{array}$

$\begin{array}{llll}\text { C } & -3.77766600 & 1.12846400 & -2.07938000\end{array}$

$\mathrm{N} \quad-3.38973900 \quad 2.27506300 \quad-1.29253900$

$\mathrm{N} \quad-4.35635800 \quad 2.13575100 \quad 0.92446500$

C $\quad-2.47243200 \quad 3.16299500-1.82375300$

C $\quad-4.19634500 \quad 2.92904500 \quad-0.26243300$

C $\quad-3.18489500 \quad 4.03717600 \quad 0.22079600$

N $\quad-2.43967600 \quad 4.28186600 \quad-0.98820000$

C $\quad-3.21906500 \quad 2.23218100 \quad 1.72627000$ 


\begin{tabular}{|c|c|c|c|}
\hline $\mathrm{N}$ & -2.44634100 & 3.26857200 & 1.22302200 \\
\hline $\mathrm{C}$ & -1.50727600 & 5.35360500 & -1.24605700 \\
\hline $\mathrm{C}$ & -1.26455200 & 3.66583700 & 1.94593200 \\
\hline $\mathrm{O}$ & -2.98025600 & 1.57034400 & 2.70852300 \\
\hline $\mathrm{O}$ & -1.85220200 & 3.02781700 & -2.85218900 \\
\hline $\mathrm{N}$ & -0.13292300 & 5.13561200 & -0.85988400 \\
\hline $\mathrm{N}$ & -0.02341500 & 3.78515300 & 1.19003600 \\
\hline C & 0.75280200 & 4.62045100 & -1.80451700 \\
\hline $\mathrm{C}$ & 0.37596000 & 5.01075500 & 0.48777700 \\
\hline C & 1.92362800 & 4.76441000 & 0.21540200 \\
\hline $\mathrm{N}$ & 1.88132200 & 4.19462800 & -1.13167700 \\
\hline $\mathrm{O}$ & 0.58892200 & 4.61384800 & -3.00330900 \\
\hline C & 2.95853700 & 3.62044000 & -1.91478400 \\
\hline $\mathrm{N}$ & 2.23561600 & 3.81331700 & 1.24924000 \\
\hline C & 1.10227300 & 3.19219600 & 1.76149800 \\
\hline $\mathrm{O}$ & 1.10829600 & 2.32952800 & 2.60875800 \\
\hline C & 3.53388400 & 3.33506800 & 1.64213100 \\
\hline $\mathrm{N}$ & 3.50322900 & 2.37903200 & -1.43454000 \\
\hline $\mathrm{N}$ & 4.01865000 & 2.18084800 & 0.92051800 \\
\hline $\mathrm{C}$ & 3.04577800 & 1.17183400 & -1.93428200 \\
\hline C & 4.52945600 & 2.20938700 & -0.43938600 \\
\hline C & 4.97529000 & 0.69092200 & -0.66583600 \\
\hline $\mathrm{N}$ & 3.81447500 & 0.15027200 & -1.38316600 \\
\hline $\mathrm{O}$ & 2.17769800 & 1.04737300 & -2.76881000 \\
\hline C & 3.77791300 & -1.12171200 & -2.07946900 \\
\hline $\mathrm{N}$ & 5.10825100 & 0.23279400 & 0.70351300 \\
\hline C & 4.53589500 & 1.11609800 & 1.62369700 \\
\hline $\mathrm{O}$ & 4.56155800 & 0.99386200 & 2.82721100 \\
\hline C & 5.35282200 & -1.11535800 & 1.16143100 \\
\hline $\mathrm{N}$ & 3.39186200 & -2.27246000 & -1.29895700 \\
\hline $\mathrm{N}$ & 4.33451800 & -2.11192200 & 0.92552300 \\
\hline $\mathrm{C}$ & 4.18627400 & -2.91642300 & -0.25467400 \\
\hline C & 3.19053100 & -2.20046000 & 1.71773600 \\
\hline $\mathrm{C}$ & 3.16978800 & -4.02061300 & 0.22912400 \\
\hline $\mathrm{N}$ & 2.42634000 & -3.24702000 & 1.22301700 \\
\hline $\mathrm{C}$ & 2.47430100 & -3.15920800 & -1.82754400 \\
\hline $\mathrm{N}$ & 2.42980400 & -4.27064700 & -0.98273100 \\
\hline $\mathrm{O}$ & 2.94269100 & -1.52835900 & 2.69064700 \\
\hline $\mathrm{O}$ & 1.86002000 & -3.02912700 & -2.86112100 \\
\hline C & 1.24832000 & -3.64456100 & 1.95122400 \\
\hline C & 1.49374300 & -5.34045400 & -1.23606600 \\
\hline $\mathrm{H}$ & -1.45851100 & 4.59811800 & 2.47963300 \\
\hline $\mathrm{H}$ & -1.09559000 & 2.87784200 & 2.68486200 \\
\hline $\mathrm{H}$ & -1.47425600 & 5.49964000 & -2.32763400 \\
\hline $\mathrm{H}$ & -1.88999100 & 6.25971800 & -0.77410100 \\
\hline $\mathrm{H}$ & 3.77405800 & 4.33070000 & -2.05988600 \\
\hline $\mathrm{H}$ & 2.50748000 & 3.41766800 & -2.88854900 \\
\hline $\mathrm{H}$ & 4.23280500 & 4.17328500 & 1.57484700 \\
\hline $\mathrm{H}$ & 3.47038100 & 3.01006400 & 2.68277200 \\
\hline $\mathrm{H}$ & 4.73646500 & -1.29255700 & -2.57837000 \\
\hline $\mathrm{H}$ & 3.00949200 & -1.02923900 & -2.85101600 \\
\hline $\mathrm{H}$ & 6.28362700 & -1.48021700 & 0.73267300 \\
\hline $\mathrm{H}$ & 5.46908800 & -1.02283700 & 2.24316400 \\
\hline $\mathrm{H}$ & 1.45880200 & -5.48826500 & -2.31733000 \\
\hline $\mathrm{H}$ & 1.87510200 & -6.24658700 & -0.76299400 \\
\hline $\mathrm{H}$ & 1.07833500 & -2.85117900 & 2.68418300 \\
\hline $\mathrm{H}$ & 1.44800000 & -4.57211800 & 2.49116100 \\
\hline $\mathrm{H}$ & -3.79414900 & -4.32836300 & -2.04794900 \\
\hline $\mathrm{H}$ & -2.52759800 & -3.41970100 & -2.88219000 \\
\hline $\mathrm{H}$ & -3.48487300 & -2.98743900 & 2.68755000 \\
\hline $\mathrm{H}$ & -4.24764800 & -4.15375900 & 1.58377900 \\
\hline
\end{tabular}




\begin{tabular}{|c|c|c|c|}
\hline $\mathrm{H}$ & -6.30159100 & 1.49407800 & 0.72014000 \\
\hline $\mathrm{H}$ & -5.49336500 & 1.04256100 & 2.23614000 \\
\hline $\mathrm{H}$ & -3.00617000 & 1.03378700 & -2.84775400 \\
\hline $\mathrm{H}$ & -4.73331500 & 1.30442100 & -2.58181300 \\
\hline $\mathrm{H}$ & -6.02877500 & -1.12996700 & -2.46470900 \\
\hline $\mathrm{H}$ & -5.17226200 & -4.22737000 & -0.55039100 \\
\hline $\mathrm{H}$ & -2.08309300 & -6.76642700 & -0.37375400 \\
\hline $\mathrm{H}$ & 1.01811100 & -6.35570700 & 1.33700600 \\
\hline $\mathrm{H}$ & 4.71843500 & -4.74087600 & 1.44826900 \\
\hline $\mathrm{H}$ & 5.88179100 & -2.61261700 & -1.47056500 \\
\hline $\mathrm{H}$ & 6.96228100 & 1.36701000 & -1.00979900 \\
\hline $\mathrm{H}$ & 5.15268400 & 4.24121100 & -0.56286800 \\
\hline $\mathrm{H}$ & 2.56907800 & 6.52942000 & 1.26304100 \\
\hline $\mathrm{H}$ & -1.03163100 & 6.36677800 & 1.32944300 \\
\hline $\mathrm{H}$ & -4.74177300 & 4.76330700 & 1.42659900 \\
\hline $\mathrm{H}$ & -5.87973200 & 2.61860900 & -1.49405100 \\
\hline C & -5.50501800 & 3.44203800 & -0.89565300 \\
\hline C & -3.99137300 & 5.22991100 & 0.78376000 \\
\hline $\mathrm{C}$ & 0.04881700 & 6.25993600 & 1.32583400 \\
\hline C & 2.66370000 & 6.12939900 & 0.25391600 \\
\hline C & 5.63680900 & 3.27025200 & -0.57041600 \\
\hline $\mathrm{C}$ & 6.28590800 & 0.67497700 & -1.50435400 \\
\hline C & 5.50175600 & -3.43309300 & -0.87124400 \\
\hline C & 3.97364100 & -5.21096600 & 0.80144300 \\
\hline C & -0.06229900 & -6.24887500 & 1.33600200 \\
\hline $\mathrm{C}$ & -2.68160300 & -6.11787200 & 0.27405800 \\
\hline C & -5.65412200 & -3.25521600 & -0.56185400 \\
\hline C & -6.29339600 & -0.66266500 & -1.50907100 \\
\hline $\mathrm{H}$ & -5.21143000 & 4.20455800 & -1.62437100 \\
\hline $\mathrm{H}$ & -6.97366400 & -1.34744400 & -1.00962100 \\
\hline $\mathrm{H}$ & -6.11536900 & -3.18205800 & -1.54875800 \\
\hline $\mathrm{H}$ & -2.58606500 & -6.51298500 & 1.28508700 \\
\hline $\mathrm{H}$ & -0.42978700 & -7.13169700 & 0.81061100 \\
\hline $\mathrm{H}$ & 4.51311500 & -5.65179700 & -0.04095400 \\
\hline $\mathrm{H}$ & 5.21556000 & -4.19946300 & -1.59879800 \\
\hline $\mathrm{H}$ & 6.02186000 & 1.13229600 & -2.46497000 \\
\hline $\mathrm{H}$ & 6.09749400 & 3.19381300 & -1.55736400 \\
\hline $\mathrm{H}$ & 2.06309900 & 6.77381400 & -0.39612200 \\
\hline $\mathrm{H}$ & 0.41460200 & 7.14276500 & 0.79921400 \\
\hline $\mathrm{H}$ & -4.52383800 & 5.67013500 & -0.06346400 \\
\hline C & 6.64464800 & -3.93515200 & 0.02673400 \\
\hline $\mathrm{H}$ & 6.66718800 & -5.02358600 & 0.10147600 \\
\hline $\mathrm{H}$ & 7.60843800 & -3.62044500 & -0.38415900 \\
\hline $\mathrm{H}$ & 6.57763200 & -3.54600000 & 1.04540800 \\
\hline $\mathrm{C}$ & 3.37798400 & -6.35949000 & 1.61496000 \\
\hline $\mathrm{C}$ & -0.53714500 & -6.25296200 & 2.79293600 \\
\hline $\mathrm{C}$ & -4.13534100 & -6.32968400 & -0.14763600 \\
\hline $\mathrm{C}$ & -6.70750600 & -3.25299000 & 0.54927900 \\
\hline C & -7.14640500 & 0.57317100 & -1.80925000 \\
\hline $\mathrm{C}$ & -6.65654700 & 3.94925800 & -0.01193600 \\
\hline C & -3.39648400 & 6.37706500 & 1.59970900 \\
\hline $\mathrm{C}$ & 0.52739800 & 6.26587100 & 2.78157200 \\
\hline C & 4.11657100 & 6.34201100 & -0.17030700 \\
\hline C & 6.69101300 & 3.27528300 & 0.54001400 \\
\hline C & 7.14452200 & -0.56062000 & -1.78922500 \\
\hline $\mathrm{H}$ & 7.14844600 & 2.29719000 & 0.70544800 \\
\hline $\mathrm{H}$ & 7.48577400 & 3.98237800 & 0.28875400 \\
\hline $\mathrm{H}$ & 6.26671600 & 3.58159900 & 1.49921000 \\
\hline $\mathrm{H}$ & 4.30954800 & 6.05423500 & -1.20469900 \\
\hline $\mathrm{H}$ & 4.32271100 & 7.41416400 & -0.10215800 \\
\hline $\mathrm{H}$ & 4.83761200 & 5.84818400 & 0.48367400 \\
\hline
\end{tabular}




$\begin{array}{lrrr}\mathrm{H} & 0.06444500 & 5.47671900 & 3.37727400 \\ \mathrm{H} & 1.60550200 & 6.12930700 & 2.88144700 \\ \mathrm{H} & 0.26859200 & 7.22195400 & 3.24408900 \\ \mathrm{H} & -2.84142000 & 6.03671400 & 2.47577800 \\ \mathrm{H} & -2.76576900 & 7.04574800 & 1.01188800 \\ \mathrm{H} & -4.22690600 & 6.98458400 & 1.97111900 \\ \mathrm{H} & -7.61648300 & 3.63921100 & -0.43514200 \\ \mathrm{H} & -6.60401900 & 3.55954900 & 1.00741000 \\ \mathrm{H} & -6.67509700 & 5.03772900 & 0.06293100 \\ \mathrm{H} & -8.08816900 & 0.22225100 & -2.24149100 \\ \mathrm{H} & -7.40656700 & 1.13927500 & -0.91309000 \\ \mathrm{H} & -6.70775400 & 1.24933200 & -2.54271800 \\ \mathrm{H} & -7.50226600 & -3.96201600 & 0.30349000 \\ \mathrm{H} & -6.28236600 & -3.55245400 & 1.51027900 \\ \mathrm{H} & -7.16518800 & -2.27395400 & 0.70833100 \\ \mathrm{H} & -4.34383000 & -7.40099400 & -0.07380500 \\ \mathrm{H} & -4.85474500 & -5.83074900 & 0.50423600 \\ \mathrm{H} & -4.32831400 & -6.04653300 & -1.18330500 \\ \mathrm{H} & -0.29616400 & -7.21614700 & 3.25030500 \\ \mathrm{H} & -0.05656300 & -5.47627400 & 3.39105300 \\ \mathrm{H} & -1.61202200 & -6.09530000 & 2.89648900 \\ \mathrm{H} & 2.75302900 & -7.03077500 & 1.02397300 \\ \mathrm{H} & 4.20879100 & -6.96374800 & 1.99085000 \\ \mathrm{H} & 2.81711500 & -6.02140000 & 2.48814700 \\ \mathrm{H} & 6.71142000 & -1.24550400 & -2.51787600 \\ \mathrm{H} & 8.08707700 & -0.21089700 & -2.22067700 \\ \mathrm{H} & 7.40232000 & -1.11699600 & -0.88631000 \\ \mathrm{C} & 0.29908100 & -0.81973200 & -0.77302400 \\ \mathrm{H} & 0.12604200 & -1.85160500 & -0.47801500 \\ \mathrm{H} & 0.44608400 & -0.63362100 & -1.83173900 \\ \mathrm{C} & 0.31624000 & 0.16179700 & 0.12285300 \\ \mathrm{H} & 0.47889000 & 1.19135400 & -0.19017500 \\ \mathrm{H} & 0.16352200 & -0.01079700 & 1.18423700\end{array}$

$\begin{array}{lrrr}\mathrm{C}_{2} \mathrm{H}_{4} @(\mathrm{OH})_{12} \mathrm{CB}[6] & & \\ \mathrm{C} & -1.65655000 & -4.75638500 & -0.02791600 \\ \mathrm{C} & -0.09837500 & -4.99230700 & -0.00884700 \\ \mathrm{~N} & 0.29592300 & -4.42282700 & 1.24637800 \\ \mathrm{C} & -0.76662300 & -3.87821900 & 1.95221000 \\ \mathrm{~N} & -1.89806100 & -3.97210300 & 1.14586600 \\ \mathrm{~N} & -1.86751200 & -4.13178300 & -1.29612100 \\ \mathrm{~N} & 0.36209900 & -4.32684000 & -1.18723200 \\ \mathrm{C} & 1.62295100 & -4.50509700 & 1.80151800 \\ \mathrm{O} & -0.72789000 & -3.44299100 & 3.07623400 \\ \mathrm{C} & 1.69128300 & -4.49178700 & -1.72818200 \\ \mathrm{C} & -0.68444900 & -3.87970700 & -1.98158800 \\ \mathrm{O} & -0.59139500 & -3.40232100 & -3.08506000 \\ \mathrm{~N} & 2.66301900 & -3.58360100 & -1.17693700 \\ \mathrm{C} & 3.34211100 & -3.78582300 & 0.06252200 \\ \mathrm{C} & 3.24001900 & -2.58806400 & -1.94669600 \\ \mathrm{C} & 4.39569100 & -2.61219600 & 0.10293400 \\ \mathrm{~N} & 2.57900400 & -3.57391500 & 1.25997100 \\ \mathrm{~N} & 4.24997500 & -2.00829200 & -1.18789900 \\ \mathrm{O} & 2.95488600 & -2.30651300 & -3.08470800 \\ \mathrm{~N} & 4.00411000 & -1.84804300 & 1.24203900 \\ \mathrm{C} & 2.97752300 & -2.44210400 & 1.95880100\end{array}$




\begin{tabular}{|c|c|c|c|}
\hline $\mathrm{O}$ & 2.54169000 & -2.07302700 & 3.02203300 \\
\hline $\mathrm{C}$ & 5.01789300 & -0.89203200 & -1.67484000 \\
\hline $\mathrm{C}$ & 4.77791300 & -0.76602700 & 1.79698300 \\
\hline $\mathrm{N}$ & 4.48010600 & 0.52380300 & 1.23013500 \\
\hline $\mathrm{N}$ & 4.61188500 & 0.40786600 & -1.20607000 \\
\hline $\mathrm{C}$ & 3.84620700 & 1.51061300 & 1.96496300 \\
\hline C & 5.04291900 & 1.01778800 & 0.01679300 \\
\hline $\mathrm{C}$ & 4.45523500 & 2.47579300 & -0.09094900 \\
\hline $\mathrm{N}$ & 3.83620900 & 2.66401100 & 1.18830400 \\
\hline C & 3.75799400 & 1.21931700 & -1.93891400 \\
\hline $\mathrm{N}$ & 3.59708700 & 2.40379600 & -1.23195700 \\
\hline $\mathrm{C}$ & 3.23262900 & 3.88633000 & 1.65138100 \\
\hline $\mathrm{C}$ & 2.97748000 & 3.55105100 & -1.84781500 \\
\hline $\mathrm{O}$ & 3.28137500 & 0.96167900 & -3.01684100 \\
\hline $\mathrm{O}$ & 3.41941600 & 1.40797600 & 3.08874100 \\
\hline $\mathrm{N}$ & 1.90354200 & 4.15604100 & 1.16424300 \\
\hline $\mathrm{N}$ & 1.71269900 & 3.91512900 & -1.25641500 \\
\hline $\mathrm{C}$ & 0.77079600 & 3.91395900 & 1.93173400 \\
\hline $\mathrm{C}$ & 1.59523300 & 4.75022800 & -0.10278900 \\
\hline $\mathrm{C}$ & 0.04727600 & 5.01523900 & -0.00085300 \\
\hline $\mathrm{N}$ & -0.33061500 & 4.31085000 & 1.18380100 \\
\hline $\mathrm{O}$ & 0.75373900 & 3.47818700 & 3.05604700 \\
\hline $\mathrm{C}$ & -1.63550300 & 4.41406800 & 1.79148600 \\
\hline $\mathrm{N}$ & -0.44462100 & 4.51749500 & -1.25117500 \\
\hline $\mathrm{C}$ & 0.53738200 & 3.87496200 & -1.99589700 \\
\hline $\mathrm{O}$ & 0.40025100 & 3.40714100 & -3.09877400 \\
\hline $\mathrm{C}$ & -1.80185600 & 4.64096000 & -1.72049600 \\
\hline $\mathrm{N}$ & -2.60606400 & 3.50264400 & 1.23783500 \\
\hline $\mathrm{N}$ & -2.73681700 & 3.68196100 & -1.19113800 \\
\hline $\mathrm{C}$ & -3.15322400 & 2.48498600 & 2.00426400 \\
\hline $\mathrm{C}$ & -3.39207900 & 3.79134000 & 0.08035000 \\
\hline $\mathrm{C}$ & -4.43516000 & 2.61226300 & 0.03954700 \\
\hline $\mathrm{N}$ & -4.24411200 & 1.97602200 & 1.30500300 \\
\hline $\mathrm{O}$ & -2.78658400 & 2.13282500 & 3.09740500 \\
\hline $\mathrm{C}$ & -4.94922200 & 0.80339500 & 1.75222600 \\
\hline $\mathrm{N}$ & -4.07356500 & 1.88303600 & -1.13938600 \\
\hline $\mathrm{C}$ & -3.17165700 & 2.59207800 & -1.93000600 \\
\hline $\mathrm{O}$ & -2.86182500 & 2.33105500 & -3.06568300 \\
\hline C & -4.91185200 & 0.86612600 & -1.73408900 \\
\hline $\mathrm{N}$ & -4.50133900 & -0.43847000 & 1.16329200 \\
\hline $\mathrm{N}$ & -4.64926100 & -0.47350100 & -1.27711000 \\
\hline $\mathrm{C}$ & -5.07914100 & -1.00196100 & -0.02000500 \\
\hline $\mathrm{C}$ & -3.81303400 & -1.33753100 & -1.97082800 \\
\hline $\mathrm{C}$ & -4.51323900 & -2.47163300 & -0.04785200 \\
\hline $\mathrm{N}$ & -3.65232400 & -2.48187100 & -1.19389200 \\
\hline C & -3.89655600 & -1.40906500 & 1.95613400 \\
\hline $\mathrm{N}$ & -3.90022500 & -2.59787100 & 1.23846800 \\
\hline $\mathrm{O}$ & -3.35070600 & -1.15052600 & -3.06826700 \\
\hline $\mathrm{O}$ & -3.48828900 & -1.25964900 & 3.08045200 \\
\hline $\mathrm{C}$ & -3.14585500 & -3.69624400 & -1.79414300 \\
\hline $\mathrm{C}$ & -3.21383600 & -3.77154700 & 1.71073200 \\
\hline $\mathrm{H}$ & 3.66246600 & 4.40098400 & -1.79767200 \\
\hline $\mathrm{H}$ & 2.77568800 & 3.28634600 & -2.88774500 \\
\hline $\mathrm{H}$ & 3.15937000 & 3.80662800 & 2.73812100 \\
\hline $\mathrm{H}$ & 3.87930900 & 4.72099800 & 1.36694400 \\
\hline $\mathrm{H}$ & -2.00365400 & 5.43819000 & 1.69227900 \\
\hline $\mathrm{H}$ & -1.51513100 & 4.15298700 & 2.84480700 \\
\hline $\mathrm{H}$ & -2.16195800 & 5.64386000 & -1.47542300 \\
\hline $\mathrm{H}$ & -1.77606900 & 4.49342000 & -2.80230200 \\
\hline $\mathrm{H}$ & -6.01446800 & 0.92940700 & 1.54416000 \\
\hline $\mathrm{H}$ & -4.77729300 & 0.72441200 & 2.82755900 \\
\hline
\end{tabular}




\begin{tabular}{lrrr}
$\mathrm{H}$ & -5.95866200 & 1.10721200 & -1.53410500 \\
$\mathrm{H}$ & -4.71524000 & 0.87934600 & -2.80802200 \\
$\mathrm{H}$ & -3.08384000 & -3.64633500 & 2.78755000 \\
$\mathrm{H}$ & -3.82525200 & -4.65135500 & 1.49728300 \\
$\mathrm{H}$ & -3.01301100 & -3.49609300 & -2.85930100 \\
$\mathrm{H}$ & -3.87822900 & -4.49321100 & -1.64363200 \\
$\mathrm{H}$ & 2.00439800 & -5.51798300 & 1.64471700 \\
$\mathrm{H}$ & 1.52920300 & -4.28849200 & 2.86787000 \\
$\mathrm{H}$ & 1.62981900 & -4.27674100 & -2.79682800 \\
$\mathrm{H}$ & 2.01985400 & -5.52040200 & -1.56266400 \\
$\mathrm{H}$ & 6.06535000 & -1.04044200 & -1.39828200 \\
$\mathrm{H}$ & 4.90462500 & -0.89033600 & -2.76098900 \\
$\mathrm{H}$ & 4.53413100 & -0.70427200 & 2.85930100 \\
$\mathrm{H}$ & 5.83980100 & -0.98405300 & 1.66255000 \\
$\mathrm{O}$ & 5.70160400 & -3.07193400 & 0.32040400 \\
$\mathrm{O}$ & 3.94275700 & -5.04665700 & 0.03185200 \\
$\mathrm{O}$ & 0.24020000 & -6.34376400 & -0.10824100 \\
$\mathrm{O}$ & -2.37697100 & -5.95156800 & 0.09755400 \\
$\mathrm{O}$ & -5.51416800 & -3.42815000 & -0.24818300 \\
$\mathrm{O}$ & -6.47082200 & -0.96406500 & 0.11751000 \\
$\mathrm{O}$ & -5.75000700 & 3.07140400 & -0.12261300 \\
$\mathrm{O}$ & -3.99710600 & 5.03812700 & 0.25471200 \\
$\mathrm{O}$ & -0.25233800 & 6.37069900 & 0.17926700 \\
$\mathrm{O}$ & 2.32547200 & 5.92120500 & -0.32873700 \\
$\mathrm{O}$ & 5.44162500 & 3.43510100 & -0.34293100 \\
$\mathrm{O}$ & 6.43660200 & 1.00646800 & 0.14488900 \\
$\mathrm{H}$ & 5.97318500 & -3.54007500 & -0.47776500 \\
$\mathrm{H}$ & 4.50244700 & -5.11175800 & 0.81520300 \\
$\mathrm{H}$ & -0.17648100 & -6.79483600 & 0.63572100 \\
$\mathrm{H}$ & -2.26803000 & -6.43478300 & -0.72969000 \\
$\mathrm{H}$ & -6.06686200 & -3.42559400 & 0.54189100 \\
$\mathrm{H}$ & -6.84266200 & -1.33242600 & -0.69245000 \\
$\mathrm{H}$ & -6.01892700 & 3.45550400 & 0.72004800 \\
$\mathrm{H}$ & -4.62718200 & 5.14597800 & -0.46827500 \\
$\mathrm{H}$ & -0.00833800 & 6.82358400 & -0.63674400 \\
$\mathrm{H}$ & 2.18816400 & 6.49018600 & 0.43831200 \\
$\mathrm{H}$ & 6.01073300 & 3.46324300 & 0.43534900 \\
$\mathrm{H}$ & 6.79663100 & 1.37311200 & -0.67175300 \\
$\mathrm{C}$ & 0.98363100 & -0.64340200 & -0.44993900 \\
$\mathrm{H}$ & 1.03371300 & -0.45939000 & -1.52000600 \\
$\mathrm{H}$ & 0.76505800 & -1.66373200 & -0.14148900 \\
$\mathrm{H}$ & 1.17729600 & 0.31936200 & 0.44595700 \\
& 1.12736100 & 0.13375000 & 1.51569300 \\
\hline & 1.39235500 & 1.34040300 & 0.13589800
\end{tabular}

\section{$\mathrm{C}_{2} \mathrm{H}_{4} @(\mathrm{CN})_{12} \mathrm{CB}[6]$}

$\begin{array}{llll}\text { C } & -0.69360300 & -4.95996800 & -0.09654500\end{array}$

$\begin{array}{llll}\mathrm{C} & 0.88387500 & -4.94325800 & 0.08979500\end{array}$

N $\quad \begin{array}{llll}\mathrm{N} & 1.03655700 & -4.08135400 & 1.23791200\end{array}$

$\begin{array}{llll}\mathrm{C} & -0.15892800 & -3.89768800 & 1.92775300\end{array}$

$\mathrm{N} \quad \quad-1.16921200 \quad-4.46595900 \quad 1.16179800$

N $\quad-0.86712000 \quad-4.08851800 \quad-1.23611900$

$\mathrm{N} \quad 1.34840100 \quad-4.42794300 \quad-1.16467100$

$\begin{array}{llll}\text { C } & 2.29424300 & -3.86385300 & 1.91207700\end{array}$

$\begin{array}{llll}\text { O } & -0.28858900 & -3.37608600 & 3.00297700\end{array}$

$\begin{array}{llll}\text { C } & 2.72596300 & -4.37269100 & -1.59427000\end{array}$ 


\begin{tabular}{|c|c|c|c|}
\hline C & 0.32566100 & -3.88004200 & -1.92810800 \\
\hline $\mathrm{O}$ & 0.44218800 & -3.35575400 & -3.00316000 \\
\hline $\mathrm{N}$ & 3.47744000 & -3.24350500 & -1.10298600 \\
\hline $\mathrm{C}$ & 4.04538400 & -3.13089300 & 0.21015900 \\
\hline C & 3.72183400 & -2.13403900 & -1.90332800 \\
\hline $\mathrm{C}$ & 4.87891900 & -1.77339100 & 0.12375600 \\
\hline $\mathrm{N}$ & 3.12669400 & -2.86406100 & 1.28933000 \\
\hline $\mathrm{N}$ & 4.45288400 & -1.22875400 & -1.14052100 \\
\hline $\mathrm{O}$ & 3.39730800 & -1.99859000 & -3.05217400 \\
\hline $\mathrm{N}$ & 4.46558000 & -1.06859100 & 1.30147900 \\
\hline $\mathrm{C}$ & 3.41440300 & -1.68285600 & 1.96600600 \\
\hline $\mathrm{O}$ & 2.87882600 & -1.28714600 & 2.96705300 \\
\hline C & 5.01911100 & -0.04844400 & -1.75115800 \\
\hline $\mathrm{C}$ & 5.00740100 & 0.18690600 & 1.75536300 \\
\hline $\mathrm{N}$ & 4.40854900 & 1.34983600 & 1.14221200 \\
\hline $\mathrm{N}$ & 4.43841600 & 1.19062900 & -1.29983600 \\
\hline C & 3.65152000 & 2.23559700 & 1.90283300 \\
\hline C & 4.82527200 & 1.90679300 & -0.12004800 \\
\hline $\mathrm{C}$ & 3.95355700 & 3.23956600 & -0.21000300 \\
\hline $\mathrm{N}$ & 3.38120600 & 3.33868500 & 1.10207600 \\
\hline C & 3.36953100 & 1.77194400 & -1.96602400 \\
\hline $\mathrm{N}$ & 3.04418500 & 2.94357800 & -1.28952400 \\
\hline C & 2.60025900 & 4.44827900 & 1.59213200 \\
\hline $\mathrm{C}$ & 2.18199800 & 3.91709400 & -1.91341900 \\
\hline $\mathrm{O}$ & 2.84806700 & 1.35980700 & -2.96787600 \\
\hline $\mathrm{O}$ & 3.32619600 & 2.09183100 & 3.05044800 \\
\hline $\mathrm{N}$ & 1.22131300 & 4.46616800 & 1.16368800 \\
\hline $\mathrm{N}$ & 0.91985800 & 4.10134500 & -1.23774300 \\
\hline $\mathrm{C}$ & 0.21229800 & 3.89837700 & 1.93109300 \\
\hline $\mathrm{C}$ & 0.74384300 & 4.96385200 & -0.09324000 \\
\hline C & -0.83367000 & 4.94009000 & 0.09253000 \\
\hline $\mathrm{N}$ & -0.98483400 & 4.07099500 & 1.23676900 \\
\hline $\mathrm{O}$ & 0.34073100 & 3.38581300 & 3.01036300 \\
\hline $\mathrm{C}$ & -2.24231600 & 3.86094100 & 1.91755100 \\
\hline $\mathrm{N}$ & -1.29550500 & 4.42683300 & -1.16303300 \\
\hline $\mathrm{C}$ & -0.27071600 & 3.87931000 & -1.92465700 \\
\hline $\mathrm{O}$ & -0.38747800 & 3.34526300 & -2.99532100 \\
\hline $\mathrm{C}$ & -2.67210800 & 4.36633100 & -1.59250400 \\
\hline $\mathrm{N}$ & -3.08640300 & 2.86921800 & 1.29960400 \\
\hline $\mathrm{N}$ & -3.41879600 & 3.23705900 & -1.09655900 \\
\hline $\mathrm{C}$ & -3.35673600 & 1.67431100 & 1.95753700 \\
\hline $\mathrm{C}$ & -3.99654000 & 3.13230100 & 0.21291500 \\
\hline C & -4.83240300 & 1.77519500 & 0.12627300 \\
\hline $\mathrm{N}$ & -4.40981200 & 1.06405800 & 1.29709200 \\
\hline $\mathrm{O}$ & -2.80555800 & 1.26840100 & 2.94730400 \\
\hline $\mathrm{C}$ & -4.95837100 & -0.18728500 & 1.75463600 \\
\hline $\mathrm{N}$ & -4.42121800 & 1.23814800 & -1.14462500 \\
\hline $\mathrm{C}$ & -3.66576400 & 2.12793300 & -1.89642500 \\
\hline $\mathrm{O}$ & -3.32456100 & 1.98505200 & -3.04012300 \\
\hline $\mathrm{C}$ & -4.97163800 & 0.04989000 & -1.74995400 \\
\hline $\mathrm{N}$ & -4.37411400 & -1.35792200 & 1.14649400 \\
\hline $\mathrm{N}$ & -4.38284500 & -1.18414900 & -1.29534100 \\
\hline C & -4.77743400 & -1.90691300 & -0.12216300 \\
\hline $\mathrm{C}$ & -3.31173600 & -1.76063000 & -1.95767900 \\
\hline $\mathrm{C}$ & -3.90342200 & -3.23895300 & -0.21309100 \\
\hline $\mathrm{N}$ & -3.00156100 & -2.94556800 & -1.29883200 \\
\hline $\mathrm{C}$ & -3.59487900 & -2.22894300 & 1.89630900 \\
\hline $\mathrm{N}$ & -3.32295100 & -3.33168900 & 1.09586200 \\
\hline $\mathrm{O}$ & -2.77686800 & -1.33881600 & -2.94958200 \\
\hline $\mathrm{O}$ & -3.25358400 & -2.07788000 & 3.03898600 \\
\hline C & -2.12859500 & -3.91047500 & -1.91880700 \\
\hline
\end{tabular}




\begin{tabular}{|c|c|c|c|}
\hline C & -2.54749000 & -4.44245300 & 1.58967300 \\
\hline $\mathrm{H}$ & 2.70549700 & 4.87743200 & -2.00243200 \\
\hline $\mathrm{H}$ & 1.94597000 & 3.53638400 & -2.91035200 \\
\hline $\mathrm{H}$ & 2.59251200 & 4.35909300 & 2.68019600 \\
\hline $\mathrm{H}$ & 3.07660600 & 5.38996800 & 1.30189800 \\
\hline $\mathrm{H}$ & -2.78634600 & 4.80969500 & 2.00663300 \\
\hline $\mathrm{H}$ & -1.99353800 & 3.48724500 & 2.91400000 \\
\hline $\mathrm{H}$ & -3.17597000 & 5.29568200 & -1.30881100 \\
\hline $\mathrm{H}$ & -2.65873100 & 4.27120000 & -2.67996000 \\
\hline $\mathrm{H}$ & -6.04226100 & -0.18019500 & 1.59407200 \\
\hline $\mathrm{H}$ & -4.74673800 & -0.26095600 & 2.82339300 \\
\hline $\mathrm{H}$ & -6.05403000 & 0.00904400 & -1.58494500 \\
\hline $\mathrm{H}$ & -4.76690500 & 0.13100100 & -2.81951900 \\
\hline $\mathrm{H}$ & -2.53863400 & -4.34987500 & 2.67741000 \\
\hline $\mathrm{H}$ & -3.02702700 & -5.38349700 & 1.30248800 \\
\hline $\mathrm{H}$ & -1.88849700 & -3.52514000 & -2.91296600 \\
\hline $\mathrm{H}$ & -2.64528100 & -4.87384000 & -2.01397700 \\
\hline $\mathrm{H}$ & 2.84531400 & -4.80909300 & 1.99625700 \\
\hline $\mathrm{H}$ & 2.04908800 & -3.49359400 & 2.91074000 \\
\hline $\mathrm{H}$ & 2.71371800 & -4.28218700 & -2.68216700 \\
\hline $\mathrm{H}$ & 3.22706800 & -5.30220500 & -1.30647100 \\
\hline $\mathrm{H}$ & 6.10149800 & -0.01681500 & -1.58399300 \\
\hline $\mathrm{H}$ & 4.81531800 & -0.13044800 & -2.82094800 \\
\hline $\mathrm{H}$ & 4.79712000 & 0.26192700 & 2.82441100 \\
\hline $\mathrm{H}$ & 6.09092000 & 0.18808100 & 1.59216500 \\
\hline $\mathrm{C}$ & -1.09007400 & 0.49250100 & -0.43897200 \\
\hline $\mathrm{H}$ & -1.06671900 & 0.34048800 & -1.51476700 \\
\hline C & -1.07333100 & -0.51992300 & 0.42375800 \\
\hline $\mathrm{H}$ & -1.02984000 & -1.54957500 & 0.07334600 \\
\hline $\mathrm{H}$ & -1.09707100 & -0.36764300 & 1.49960100 \\
\hline $\mathrm{H}$ & -1.12410900 & 1.52262100 & -0.08871400 \\
\hline $\mathrm{C}$ & 1.29852300 & 6.30957800 & -0.36270700 \\
\hline C & -1.42797600 & 6.26869300 & 0.36184100 \\
\hline $\mathrm{C}$ & -4.81986900 & 4.32726900 & 0.50865700 \\
\hline C & -6.30146300 & 1.96658800 & 0.14599800 \\
\hline $\mathrm{C}$ & -6.24031300 & -2.14150800 & -0.13250100 \\
\hline C & -4.69284900 & -4.45565400 & -0.51279200 \\
\hline C & -1.25251000 & -6.30218900 & -0.37352300 \\
\hline C & 1.47377400 & -6.27506800 & 0.35381300 \\
\hline $\mathrm{C}$ & 4.87049800 & -4.32234500 & 0.51594700 \\
\hline C & 6.34806000 & -1.96379600 & 0.12926500 \\
\hline $\mathrm{C}$ & 6.28819900 & 2.14003400 & -0.11790900 \\
\hline C & 4.74513600 & 4.45308600 & -0.51761600 \\
\hline $\mathrm{N}$ & -5.39840800 & 5.30011800 & 0.74642000 \\
\hline $\mathrm{N}$ & -7.45590400 & 2.03712500 & 0.13890300 \\
\hline $\mathrm{N}$ & -7.39206700 & -2.24640700 & -0.11741900 \\
\hline $\mathrm{N}$ & -5.24419800 & -5.44328500 & -0.75416900 \\
\hline $\mathrm{N}$ & -1.75050700 & -7.31760600 & -0.61530000 \\
\hline $\mathrm{N}$ & 1.99616400 & -7.28043600 & 0.58598200 \\
\hline $\mathrm{N}$ & 5.45073500 & -5.29220400 & 0.76174200 \\
\hline $\mathrm{N}$ & 7.50249500 & -2.03243800 & 0.11062700 \\
\hline $\mathrm{N}$ & 7.43997100 & 2.24288000 & -0.09289700 \\
\hline $\mathrm{N}$ & 5.29858600 & 5.43801100 & -0.76522600 \\
\hline $\mathrm{N}$ & 1.79395800 & 7.32766900 & -0.59849700 \\
\hline $\mathrm{N}$ & -1.95318200 & 7.27158200 & 0.59828500 \\
\hline
\end{tabular}




\begin{tabular}{|c|c|c|c|}
\hline $\mathrm{C}$ & 2.57268300 & -4.34349000 & -0.37930800 \\
\hline $\mathrm{C}$ & 3.82564400 & -3.37953000 & -0.22981300 \\
\hline $\mathrm{N}$ & 3.60407200 & -2.80021300 & 1.08735300 \\
\hline $\mathrm{C}$ & 2.70204400 & -3.53303300 & 1.83609800 \\
\hline $\mathrm{N}$ & 2.09392500 & -4.43945800 & 0.98117700 \\
\hline $\mathrm{N}$ & 1.70882200 & -3.58686500 & -1.27887000 \\
\hline $\mathrm{N}$ & 3.64383700 & -2.46142700 & -1.32441400 \\
\hline C & 4.49924000 & -1.89577200 & 1.76408900 \\
\hline $\mathrm{O}$ & 2.52730300 & -3.45133100 & 3.03033500 \\
\hline $\mathrm{C}$ & 4.58302900 & -1.47541200 & -1.78471200 \\
\hline $\mathrm{C}$ & 2.38579300 & -2.54323500 & -1.89405300 \\
\hline $\mathrm{O}$ & 1.98501600 & -1.87297600 & -2.81744100 \\
\hline $\mathrm{N}$ & 4.54590000 & -0.21180000 & -1.08908200 \\
\hline $\mathrm{C}$ & 5.14349700 & 0.05535100 & 0.21336800 \\
\hline $\mathrm{C}$ & 4.37856500 & 0.94721100 & -1.82493000 \\
\hline $\mathrm{C}$ & 4.84002000 & 1.60120500 & 0.39503100 \\
\hline $\mathrm{N}$ & 4.44154300 & -0.52708100 & 1.32721600 \\
\hline $\mathrm{N}$ & 4.55908300 & 2.01792800 & -0.95976500 \\
\hline $\mathrm{O}$ & 4.18342700 & 1.01976600 & -3.01616600 \\
\hline $\mathrm{N}$ & 3.68087000 & 1.57863900 & 1.28432100 \\
\hline $\mathrm{C}$ & 3.52369400 & 0.34013900 & 1.89136300 \\
\hline $\mathrm{O}$ & 2.77223500 & 0.07673300 & 2.80185700 \\
\hline $\mathrm{C}$ & 4.22783000 & 3.35169300 & -1.39643100 \\
\hline $\mathrm{C}$ & 3.15942500 & 2.72824300 & 1.98526400 \\
\hline $\mathrm{N}$ & 2.19574000 & 3.50479600 & 1.25217700 \\
\hline $\mathrm{N}$ & 2.91535800 & 3.84316200 & -1.05454200 \\
\hline $\mathrm{C}$ & 0.99664400 & 3.82150300 & 1.86846700 \\
\hline $\mathrm{C}$ & 2.51358600 & 4.45189300 & 0.18845700 \\
\hline $\mathrm{C}$ & 1.07589400 & 4.99088300 & -0.15675100 \\
\hline $\mathrm{N}$ & 0.36802200 & 4.77724000 & 1.08009900 \\
\hline $\mathrm{C}$ & 1.81881100 & 3.54855200 & -1.85531200 \\
\hline $\mathrm{N}$ & 0.70140400 & 4.08181200 & -1.23512800 \\
\hline $\mathrm{C}$ & -0.94546600 & 5.26375200 & 1.42327900 \\
\hline $\mathrm{C}$ & -0.53277900 & 4.13092600 & -1.97272300 \\
\hline $\mathrm{O}$ & 1.84869600 & 2.98688800 & -2.92523900 \\
\hline $\mathrm{O}$ & 0.59564400 & 3.39666400 & 2.92655700 \\
\hline $\mathrm{N}$ & -2.06818400 & 4.48121500 & 0.97038100 \\
\hline $\mathrm{N}$ & -1.68357500 & 3.59967900 & -1.28113700 \\
\hline $\mathrm{C}$ & -2.66172600 & 3.56616000 & 1.82837300 \\
\hline $\mathrm{C}$ & -2.54580700 & 4.37065300 & -0.38845200 \\
\hline $\mathrm{C}$ & -3.79806300 & 3.40985200 & -0.22721800 \\
\hline $\mathrm{N}$ & -3.55773200 & 2.82151900 & 1.08320100 \\
\hline $\mathrm{O}$ & -2.48084100 & 3.48819100 & 3.02154500 \\
\hline $\mathrm{C}$ & -4.44605000 & 1.91622300 & 1.77082200 \\
\hline $\mathrm{N}$ & -3.63180300 & 2.49923700 & -1.33036300 \\
\hline $\mathrm{C}$ & -2.36935500 & 2.55819900 & -1.88811300 \\
\hline $\mathrm{O}$ & -1.96766700 & 1.86853700 & -2.79901400 \\
\hline $\mathrm{C}$ & -4.57371500 & 1.50912800 & -1.77736500 \\
\hline $\mathrm{N}$ & -4.40153700 & 0.54847100 & 1.33166700 \\
\hline $\mathrm{N}$ & -4.52506400 & 0.24699000 & -1.08331400 \\
\hline $\mathrm{C}$ & -3.50590700 & -0.33518200 & 1.90534300 \\
\hline $\mathrm{C}$ & -5.11503800 & -0.02567800 & 0.22019200 \\
\hline $\mathrm{C}$ & -4.82187400 & -1.57648300 & 0.39211500 \\
\hline $\mathrm{N}$ & -3.68657900 & -1.57084800 & 1.30612300 \\
\hline $\mathrm{O}$ & -2.75681400 & -0.08536300 & 2.82259100 \\
\hline $\mathrm{C}$ & -3.16553100 & -2.72841400 & 1.98934500 \\
\hline $\mathrm{N}$ & -4.51244500 & -1.98162500 & -0.96043800 \\
\hline $\mathrm{C}$ & -4.32606300 & -0.90763900 & -1.81664800 \\
\hline $\mathrm{O}$ & -4.10526500 & -0.97204400 & -3.00455200 \\
\hline $\mathrm{C}$ & -4.18748400 & -3.31367900 & -1.40517100 \\
\hline $\mathrm{N}$ & -2.19494500 & -3.49777000 & 1.25806000 \\
\hline
\end{tabular}




\begin{tabular}{|c|c|c|c|}
\hline $\mathrm{N}$ & -2.88296400 & -3.81978100 & -1.05792000 \\
\hline C & -2.49503300 & -4.43778800 & 0.18538500 \\
\hline $\mathrm{C}$ & -1.77914700 & -3.51849000 & -1.84409000 \\
\hline $\mathrm{C}$ & -1.05162800 & -4.97562700 & -0.14849100 \\
\hline $\mathrm{N}$ & -0.67494600 & -4.08618800 & -1.23924100 \\
\hline C & -0.97412400 & -3.76194700 & 1.84863000 \\
\hline $\mathrm{N}$ & -0.34454500 & -4.73454300 & 1.08504000 \\
\hline $\mathrm{O}$ & -1.79731300 & -2.92959200 & -2.90101500 \\
\hline $\mathrm{O}$ & -0.55357400 & -3.28132000 & 2.87698700 \\
\hline C & 0.56352700 & -4.13142900 & -1.96764100 \\
\hline C & 0.96875100 & -5.21329400 & 1.44083200 \\
\hline $\mathrm{H}$ & -0.73871900 & 5.15248300 & -2.31571000 \\
\hline $\mathrm{H}$ & -0.37663600 & 3.49854100 & -2.85057500 \\
\hline $\mathrm{H}$ & -1.01057700 & 5.25581900 & 2.51346500 \\
\hline $\mathrm{H}$ & -1.04262200 & 6.29135900 & 1.06325800 \\
\hline $\mathrm{H}$ & -5.48231500 & 2.26643600 & 1.72153600 \\
\hline $\mathrm{H}$ & -4.11767700 & 1.93148700 & 2.81276300 \\
\hline $\mathrm{H}$ & -5.57826100 & 1.94264100 & -1.74084400 \\
\hline $\mathrm{H}$ & -4.32845900 & 1.27559400 & -2.81601300 \\
\hline $\mathrm{H}$ & -3.98994600 & -3.37139000 & 2.32226800 \\
\hline $\mathrm{H}$ & -2.64139300 & -2.35374300 & 2.87263100 \\
\hline $\mathrm{H}$ & -4.94218400 & -4.01772800 & -1.04514100 \\
\hline $\mathrm{H}$ & -4.22737200 & -3.27003500 & -2.49563300 \\
\hline $\mathrm{H}$ & 1.03117200 & -5.18756200 & 2.53090000 \\
\hline $\mathrm{H}$ & 1.06545600 & -6.24716200 & 1.09885900 \\
\hline $\mathrm{H}$ & 0.40714900 & -3.50689800 & -2.85103000 \\
\hline $\mathrm{H}$ & 0.77625400 & -5.15502000 & -2.30028400 \\
\hline $\mathrm{H}$ & 5.53625800 & -2.23984100 & 1.69581100 \\
\hline $\mathrm{H}$ & 4.18836100 & -1.91587000 & 2.81124400 \\
\hline $\mathrm{H}$ & 4.32430500 & -1.24061200 & -2.81991000 \\
\hline $\mathrm{H}$ & 5.58767100 & -1.91016200 & -1.76279800 \\
\hline $\mathrm{H}$ & 4.97352000 & 4.05855500 & -1.02338200 \\
\hline $\mathrm{H}$ & 4.27739200 & 3.31808700 & -2.48688100 \\
\hline $\mathrm{H}$ & 2.62715200 & 2.34415600 & 2.85955200 \\
\hline $\mathrm{H}$ & 3.98377900 & 3.36290100 & 2.33435300 \\
\hline C & -0.70965600 & -0.51455700 & -0.79411200 \\
\hline $\mathrm{H}$ & -0.88435200 & -0.37665600 & -1.84021200 \\
\hline C & -0.50380700 & -0.66032400 & 0.38521000 \\
\hline $\mathrm{H}$ & -0.33536600 & -0.77898400 & 1.43478600 \\
\hline $\mathrm{H}$ & 6.23833600 & 2.01539200 & 1.99424600 \\
\hline $\mathrm{H}$ & 6.77659100 & -1.36941100 & 0.02874000 \\
\hline $\mathrm{H}$ & 5.24419000 & -4.83920000 & 0.50039100 \\
\hline $\mathrm{H}$ & 2.02534200 & -6.35288400 & -1.01742400 \\
\hline $\mathrm{H}$ & -1.68570100 & -6.58643900 & -1.42905400 \\
\hline $\mathrm{H}$ & -4.42568100 & -5.07348900 & 0.95804800 \\
\hline $\mathrm{H}$ & -6.86469600 & -2.30401100 & 0.30276100 \\
\hline $\mathrm{H}$ & -6.74051800 & 1.41212100 & 0.06243900 \\
\hline $\mathrm{H}$ & -5.32060000 & 4.59776100 & -1.23135200 \\
\hline $\mathrm{H}$ & -1.99621800 & 6.36866500 & -1.05950200 \\
\hline $\mathrm{H}$ & 1.72493500 & 6.57685100 & -1.46177500 \\
\hline $\mathrm{H}$ & 4.43157200 & 5.08485300 & 0.99532500 \\
\hline $\mathrm{C}$ & 3.48756100 & 5.52471300 & 0.66863300 \\
\hline C & 1.04612400 & 6.44668200 & -0.61534300 \\
\hline C & -2.88482500 & 5.74117100 & -0.97588400 \\
\hline $\mathrm{C}$ & -5.15111700 & 4.12173500 & -0.26423000 \\
\hline $\mathrm{C}$ & -6.59912900 & 0.34305900 & 0.23253000 \\
\hline $\mathrm{C}$ & -5.99650500 & -2.37459200 & 0.95924100 \\
\hline $\mathrm{C}$ & -3.47594300 & -5.51246600 & 0.64686300 \\
\hline C & -1.01219300 & -6.43885300 & -0.58141200 \\
\hline C & 2.91295600 & -5.72233300 & -0.94735300 \\
\hline C & 5.18069000 & -4.08693600 & -0.28900600 \\
\hline
\end{tabular}




$\begin{array}{lrrr}\mathrm{C} & 6.62983800 & -0.30342700 & 0.21348400 \\ \mathrm{C} & 5.99945700 & 2.39475600 & 0.99753900 \\ \mathrm{H} & 3.70936500 & 6.24234300 & -0.12326800 \\ \mathrm{H} & 3.05828000 & 6.04887500 & 1.52595000 \\ \mathrm{H} & 6.88257500 & 2.33039500 & 0.36073100 \\ \mathrm{H} & 5.74888900 & 3.45201200 & 1.09769000 \\ \mathrm{H} & 7.14305700 & 0.23704300 & -0.58532300 \\ \mathrm{H} & 7.09380700 & -0.06742000 & 1.17245900 \\ \mathrm{H} & 5.33683900 & -4.56080700 & -1.25944100 \\ \mathrm{H} & 5.99822900 & -3.38117200 & -0.13017100 \\ \mathrm{H} & 3.33130800 & -5.62668000 & -1.95233900 \\ \mathrm{H} & 3.62510500 & -6.23942000 & -0.30325200 \\ \mathrm{H} & -0.01140100 & -6.72831500 & -0.90696300 \\ \mathrm{H} & -1.30387100 & -7.10228400 & 0.23479000 \\ \mathrm{H} & -3.68437300 & -6.22850300 & -0.15004200 \\ \mathrm{H} & -3.06106700 & -6.03786100 & 1.51041300 \\ \mathrm{H} & -5.74764300 & -3.43277800 & 1.05501400 \\ \mathrm{H} & -6.25812300 & -2.00604900 & 1.95418000 \\ \mathrm{H} & -7.12062100 & -0.18382000 & -0.56994200 \\ \mathrm{H} & -7.05912700 & 0.09808900 & 1.19111000 \\ \mathrm{H} & -5.96867900 & 3.41896700 & -0.09306300 \\ \mathrm{H} & -5.19848100 & 4.87314900 & 0.52714600 \\ \mathrm{H} & -3.30750000 & 5.63022800 & -1.97752000 \\ \mathrm{H} & -3.59358200 & 6.26944900 & -0.33714400 \\ \mathrm{H} & 0.04859600 & 6.73688400 & -0.95021100 \\ \mathrm{H} & 1.33768700 & 7.12176600 & 0.19123700\end{array}$

$\begin{array}{lccc} & * * * * * * * * * * * * * * * * * * * * * * * * * * * * * * * * * * * * * * * * * * * * * * * & \\ & & & \\ \mathrm{C}_{2} \mathrm{H}_{2} @\left(\mathrm{C}_{2} \mathrm{H}_{5}\right)_{12} \mathrm{CB}[6] & & \\ & & & \\ \mathrm{C} & 0.13131400 & -5.01517400 & -0.49330800 \\ \mathrm{C} & 1.69185000 & -4.84226600 & -0.22550000 \\ \mathrm{~N} & 1.68598000 & -4.30465500 & 1.13455400 \\ \mathrm{C} & 0.53582400 & -4.66815400 & 1.80338300 \\ \mathrm{~N} & -0.37745300 & -5.12735300 & 0.85676800 \\ \mathrm{~N} & -0.21590300 & -3.77126700 & -1.18537800 \\ \mathrm{~N} & 2.03991600 & -3.88090100 & -1.23723300 \\ \mathrm{C} & 2.78313200 & -3.76708100 & 1.91307300 \\ \mathrm{O} & 0.37318100 & -4.65952000 & 3.00302700 \\ \mathrm{C} & 3.35604100 & -3.47265200 & -1.64633600 \\ \mathrm{C} & 0.93073100 & -3.20430100 & -1.73664600 \\ \mathrm{O} & 0.97365400 & -2.33036000 & -2.57079200 \\ \mathrm{~N} & 3.91720000 & -2.35627000 & -0.92004200 \\ \mathrm{C} & 4.41748400 & -2.42263900 & 0.44173600 \\ \mathrm{C} & 4.49822400 & -1.31941700 & -1.61360700 \\ \mathrm{C} & 4.93600100 & -0.93058600 & 0.68136000 \\ \mathrm{~N} & 3.37600200 & -2.54840400 & 1.42843400 \\ \mathrm{~N} & 5.11170700 & -0.47378600 & -0.68339600 \\ \mathrm{O} & 4.53981600 & -1.19150600 & -2.81610700 \\ \mathrm{~N} & 3.78944400 & -0.33667900 & 1.38083100 \\ \mathrm{C} & 2.95891900 & -1.32103700 & 1.91013000 \\ \mathrm{O} & 2.07175100 & -1.14960500 & 2.71721300 \\ \mathrm{C} & 5.43977600 & 0.86061200 & -1.13069400 \\ \mathrm{C} & 3.80208700 & 0.93322300 & 2.08254500 \\ \mathrm{~N} & 3.48783600 & 2.10145500 & 1.29590200 \\ \mathrm{~N} & 4.47459900 & 1.91259700 & -0.90899100 \\ \mathrm{C} & 2.61702100 & 3.03926300 & 1.82165700 \\ \mathrm{C} & 4.34207000 & 2.71199100 & 0.27742700 \\ & & & \end{array}$




\begin{tabular}{|c|c|c|c|}
\hline $\mathrm{C}$ & 3.39584700 & 3.87169200 & -0.21484500 \\
\hline $\mathrm{N}$ & 2.65194900 & 4.15709800 & 0.98543300 \\
\hline $\mathrm{C}$ & 3.35256700 & 2.07032800 & -1.72357900 \\
\hline $\mathrm{N}$ & 2.62870800 & 3.14205300 & -1.22402800 \\
\hline $\mathrm{C}$ & 1.78513500 & 5.28451900 & 1.23595500 \\
\hline $\mathrm{C}$ & 1.46118300 & 3.58700200 & -1.94201700 \\
\hline $\mathrm{O}$ & 3.09137100 & 1.42589800 & -2.71170500 \\
\hline $\mathrm{O}$ & 1.98453900 & 2.93825400 & 2.84636800 \\
\hline $\mathrm{N}$ & 0.40041400 & 5.14690800 & 0.85010500 \\
\hline $\mathrm{N}$ & 0.23183000 & 3.77348900 & -1.18093000 \\
\hline $\mathrm{C}$ & -0.51147800 & 4.68691900 & 1.79983400 \\
\hline $\mathrm{C}$ & -0.11044400 & 5.02687200 & -0.49743600 \\
\hline $\mathrm{C}$ & -1.66998900 & 4.86051600 & -0.22584000 \\
\hline $\mathrm{N}$ & -1.66267700 & 4.32165300 & 1.13349200 \\
\hline $\mathrm{O}$ & -0.34479500 & 4.67987700 & 2.99845400 \\
\hline $\mathrm{C}$ & -2.75776400 & 3.77900300 & 1.91514300 \\
\hline $\mathrm{N}$ & -2.02256100 & 3.90032700 & -1.23836900 \\
\hline $\mathrm{C}$ & -0.91984000 & 3.20769300 & -1.72165000 \\
\hline $\mathrm{O}$ & -0.96834300 & 2.31344100 & -2.53656800 \\
\hline $\mathrm{C}$ & -3.34175300 & 3.49482800 & -1.64358700 \\
\hline $\mathrm{N}$ & -3.35208700 & 2.56288500 & 1.42828900 \\
\hline $\mathrm{N}$ & -3.89800200 & 2.37700200 & -0.91968300 \\
\hline $\mathrm{C}$ & -2.95554900 & 1.33448200 & 1.93093500 \\
\hline $\mathrm{C}$ & -4.39511900 & 2.44106500 & 0.44342700 \\
\hline $\mathrm{C}$ & -4.91936100 & 0.94978500 & 0.67999600 \\
\hline $\mathrm{N}$ & -3.78297200 & 0.35398700 & 1.38865700 \\
\hline $\mathrm{O}$ & -2.09381300 & 1.16407100 & 2.76281700 \\
\hline $\mathrm{C}$ & -3.80005600 & -0.91779800 & 2.08364900 \\
\hline $\mathrm{N}$ & -5.08680100 & 0.49180400 & -0.68641600 \\
\hline $\mathrm{C}$ & -4.47303200 & 1.33757700 & -1.61412500 \\
\hline $\mathrm{O}$ & -4.50834600 & 1.20995700 & -2.81729900 \\
\hline $\mathrm{C}$ & -5.41215800 & -0.84264200 & -1.13503400 \\
\hline $\mathrm{N}$ & -3.48566800 & -2.08841100 & 1.30168000 \\
\hline $\mathrm{N}$ & -4.45053200 & -1.89669400 & -0.90871400 \\
\hline $\mathrm{C}$ & -4.32985100 & -2.69938600 & 0.27775800 \\
\hline $\mathrm{C}$ & -3.31677400 & -2.04836300 & -1.70654200 \\
\hline $\mathrm{C}$ & -3.38181700 & -3.86111000 & -0.21029500 \\
\hline $\mathrm{N}$ & -2.61772800 & -3.14165100 & -1.22740900 \\
\hline $\mathrm{C}$ & -2.57759700 & -2.99997900 & 1.80105400 \\
\hline $\mathrm{N}$ & -2.62884900 & -4.13308700 & 0.98792600 \\
\hline $\mathrm{O}$ & -3.03027800 & -1.38036100 & -2.67376400 \\
\hline $\mathrm{O}$ & -1.90140200 & -2.86978400 & 2.79592600 \\
\hline $\mathrm{C}$ & -1.44833000 & -3.59075200 & -1.93972300 \\
\hline $\mathrm{C}$ & -1.76157100 & -5.25866900 & 1.24516500 \\
\hline $\mathrm{H}$ & 1.69483100 & 4.50372300 & -2.48636400 \\
\hline $\mathrm{H}$ & 1.25227300 & 2.80079200 & -2.67216800 \\
\hline $\mathrm{H}$ & 1.75966400 & 5.44067100 & 2.31633200 \\
\hline $\mathrm{H}$ & 2.22199600 & 6.16268100 & 0.75805000 \\
\hline $\mathrm{H}$ & -3.54553000 & 4.51815400 & 2.07127900 \\
\hline $\mathrm{H}$ & -2.31029500 & 3.55107400 & 2.88507000 \\
\hline $\mathrm{H}$ & -3.99015900 & 4.37383900 & -1.58692400 \\
\hline $\mathrm{H}$ & -3.29008400 & 3.16091200 & -2.68199800 \\
\hline $\mathrm{H}$ & -4.75774000 & -1.04229800 & 2.59708100 \\
\hline $\mathrm{H}$ & -3.01643000 & -0.86051000 & 2.84311600 \\
\hline $\mathrm{H}$ & -6.35652200 & -1.15217700 & -0.69219500 \\
\hline $\mathrm{H}$ & -5.53786500 & -0.74656300 & -2.21540600 \\
\hline $\mathrm{H}$ & -1.73427900 & -5.40834400 & 2.32633300 \\
\hline $\mathrm{H}$ & -2.20146300 & -6.13918700 & 0.77455600 \\
\hline $\mathrm{H}$ & -1.24110500 & -2.81093200 & -2.67689000 \\
\hline $\mathrm{H}$ & -1.68530600 & -4.51149600 & -2.47576900 \\
\hline $\mathrm{H}$ & 3.57401300 & -4.50439700 & 2.06084800 \\
\hline
\end{tabular}




\begin{tabular}{|c|c|c|c|}
\hline $\mathrm{H}$ & 2.34125100 & -3.54566500 & 2.88692500 \\
\hline $\mathrm{H}$ & 3.29665000 & -3.13234000 & -2.68228800 \\
\hline $\mathrm{H}$ & 4.00807100 & -4.34970200 & -159847200 \\
\hline $\mathrm{H}$ & 6.38170500 & 1.17009400 & -0.68266900 \\
\hline $\mathrm{H}$ & 5.57012600 & 0.76507100 & -2.21062400 \\
\hline $\mathrm{H}$ & 3.01531900 & 0.87607300 & 2.83890600 \\
\hline $\mathrm{H}$ & 4.75803100 & 1.05520700 & 2.59969300 \\
\hline $\mathrm{C}$ & -0.61918600 & -0.27728300 & -0.53209100 \\
\hline $\mathrm{H}$ & -0.80753300 & -0.02417400 & -1.55384600 \\
\hline $\mathrm{C}$ & -0.39804800 & -0.56069500 & 0.61904500 \\
\hline $\mathrm{H}$ & -0.19539400 & -0.79159500 & $1.6435210 c$ \\
\hline $\mathrm{H}$ & 5.93327600 & -1.44952700 & 2.48588200 \\
\hline $\mathrm{H}$ & 4.94072000 & -4.48314200 & 0.55037000 \\
\hline $\mathrm{H}$ & 1.74404300 & -6.86946800 & 0.33790200 \\
\hline $\mathrm{H}$ & -1.33938500 & -6.30668400 & -1.33342000 \\
\hline $\mathrm{H}$ & -4.98687600 & -4.51554900 & -1.39096900 \\
\hline $\mathrm{H}$ & -5.98618400 & -2.30258500 & 1.52321300 \\
\hline $\mathrm{H}$ & -6.87372800 & 1.71664700 & 1.02280700 \\
\hline $\mathrm{H}$ & -4.91347600 & 4.50332200 & 0.56197000 \\
\hline $\mathrm{H}$ & -2.24401300 & 6.62878400 & -1.31561300 \\
\hline $\mathrm{H}$ & 1.36201500 & 6.30146600 & -1.35783500 \\
\hline $\mathrm{H}$ & 5.00352400 & 4.51224600 & -1.40124000 \\
\hline $\mathrm{H}$ & 5.98935700 & 2.32188800 & 1.53821100 \\
\hline $\mathrm{C}$ & 5.66622100 & 3.15899800 & 0.92861100 \\
\hline $\mathrm{C}$ & 4.26773900 & 5.01961400 & -0.77268000 \\
\hline $\mathrm{C}$ & 0.27739400 & 6.24672600 & -1.35278400 \\
\hline $\mathrm{C}$ & -2.34645600 & 6.25684000 & -0.29647900 \\
\hline $\mathrm{C}$ & -5.44540300 & 3.55796900 & 0.57436600 \\
\hline C & -6.22329400 & 1.00613700 & 1.52645000 \\
\hline $\mathrm{C}$ & -5.66017400 & -3.14207300 & 0.91846100 \\
\hline $\mathrm{C}$ & -4.25155500 & -5.01582800 & -0.75630000 \\
\hline $\mathrm{C}$ & -0.25503800 & -6.24482000 & -1.33615800 \\
\hline $\mathrm{C}$ & 2.37250500 & -6.23703200 & -0.29696500 \\
\hline $\mathrm{C}$ & 5.47051500 & -3.53675600 & 0.56951800 \\
\hline $\mathrm{C}$ & 6.23202700 & -0.98453400 & 1.53914900 \\
\hline $\mathrm{H}$ & 5.40062900 & 3.94016000 & 1.64827700 \\
\hline $\mathrm{H}$ & 6.88693500 & -1.69401500 & 1.03978900 \\
\hline $\mathrm{H}$ & 5.93057500 & -3.49185200 & 1.55870400 \\
\hline $\mathrm{H}$ & 2.26447600 & -6.61068500 & -1.31483200 \\
\hline $\mathrm{H}$ & 0.07629600 & -7.14505800 & -0.81658000 \\
\hline $\mathrm{H}$ & -4.79361600 & -5.43098200 & 0.09762000 \\
\hline $\mathrm{H}$ & -5.40168300 & -3.92166200 & 1.64235000 \\
\hline $\mathrm{H}$ & -5.93173100 & 1.46931900 & 2.47623700 \\
\hline $\mathrm{H}$ & -5.90705000 & 3.50875200 & 1.56252900 \\
\hline $\mathrm{H}$ & -1.71201800 & 6.88837300 & 0.33337900 \\
\hline $\mathrm{H}$ & -0.04399200 & 7.15327900 & -0.83797900 \\
\hline $\mathrm{H}$ & 4.80940600 & 5.44210600 & 0.07789800 \\
\hline C & -6.84263700 & -3.58395400 & 0.04038800 \\
\hline $\mathrm{H}$ & -6.92735000 & -4.66980900 & -0.02726100 \\
\hline $\mathrm{H}$ & -7.78139200 & -3.21321500 & 0.46242700 \\
\hline $\mathrm{H}$ & -6.76847000 & -3.20454600 & -0.98155500 \\
\hline $\mathrm{C}$ & -3.71661100 & -6.18652400 & -1.57999800 \\
\hline C & 0.21487000 & -6.26155700 & -2.79500400 \\
\hline $\mathrm{C}$ & 3.81208400 & -6.52013900 & 0.13020500 \\
\hline $\mathrm{C}$ & 6.52719900 & -3.58129600 & -0.53744200 \\
\hline $\mathrm{C}$ & 7.13732100 & 0.20640200 & 1.86549200 \\
\hline $\mathrm{C}$ & 6.85504900 & 3.60185000 & 0.05954600 \\
\hline $\mathrm{C}$ & 3.73487100 & 6.18260500 & -1.60843300 \\
\hline C & -0.20146100 & 6.25581900 & -2.80869900 \\
\hline $\mathrm{C}$ & -3.78223300 & 6.54217700 & 0.1413010 \\
\hline $\mathrm{C}$ & -6.50102400 & 3.61173100 & -0.5334580 \\
\hline
\end{tabular}




$\begin{array}{lrrr}\mathrm{C} & -7.13214000 & -0.18429800 & 1.84459300 \\ \mathrm{H} & -7.00420900 & 2.65565900 & -0.69552800 \\ \mathrm{H} & -7.26084000 & 4.35618600 & -0.28240900 \\ \mathrm{H} & -6.06383100 & 3.89469600 & -1.49417000 \\ \mathrm{H} & -3.97328400 & 6.27323000 & 1.18112900 \\ \mathrm{H} & -3.93998900 & 7.62190500 & 0.06455600 \\ \mathrm{H} & -4.53394200 & 6.07598100 & -0.49828900 \\ \mathrm{H} & 0.24593200 & 5.45498700 & -3.40056200 \\ \mathrm{H} & -1.28186900 & 6.13948000 & -2.90819300 \\ \mathrm{H} & 0.07652800 & 7.20418700 & -3.27587200 \\ \mathrm{H} & 3.17063900 & 5.85484500 & -2.48349000 \\ \mathrm{H} & 3.13097300 & 6.88749100 & -1.03491900 \\ \mathrm{H} & 4.59518600 & 6.74528700 & -1.98219200 \\ \mathrm{H} & 7.79105700 & 3.23526100 & 0.49113600 \\ \mathrm{H} & 6.79146100 & 3.21947300 & -0.96194700 \\ \mathrm{H} & 6.93730800 & 4.68771300 & -0.01050500 \\ \mathrm{H} & 8.05347300 & -0.19354300 & 2.30999300 \\ \mathrm{H} & 7.44061000 & 0.76759800 & 0.97993900 \\ \mathrm{H} & 6.71916400 & 0.89627100 & 2.59821100 \\ \mathrm{H} & 7.28384700 & -4.33161500 & -0.29452800 \\ \mathrm{H} & 6.08917500 & -3.85067900 & -1.50167000 \\ \mathrm{H} & 7.03395300 & -2.62510200 & -0.68734800 \\ \mathrm{H} & 3.97124100 & -7.59942300 & 0.04980900 \\ \mathrm{H} & 4.55830700 & -6.05124700 & -0.51379300 \\ \mathrm{H} & 4.01049600 & -6.25378500 & 1.16935200 \\ \mathrm{H} & -0.04154100 & -7.22265200 & -3.24834100 \\ \mathrm{H} & -0.25587900 & -5.48007900 & -3.39448800 \\ \mathrm{H} & 1.29138500 & -6.11906700 & -2.90282500 \\ \mathrm{H} & -3.11141200 & -6.88438300 & -0.99934500 \\ \mathrm{H} & -4.57600200 & -6.75460500 & -1.94775500 \\ \mathrm{H} & -3.15281200 & -5.86702100 & -2.45831300 \\ \mathrm{H} & -6.71934300 & -0.87600100 & 2.57860000 \\ \mathrm{H} & -8.05082200 & 0.21565100 & 2.28392000 \\ \mathrm{H} & -7.43016100 & -0.74346900 & 0.95595100 \\ & & & \\ & & & \\ & & & \end{array}$

$\begin{array}{lrrr}* & * * * * * * * * * * * * * * * * * * * * * * * * * * * * * * * * * * * * * * * * * * * * * * * * * * * * * * * * * * * * * * * * * * * * * * * * * * * * * \\ \mathrm{C}_{2} \mathrm{H}_{2} @(\mathrm{OH})_{12} \mathrm{CB}[6] & & \\ & & & \\ \mathrm{C} & -3.97942700 & 3.16001400 & -0.03506200 \\ \mathrm{C} & -4.79257500 & 1.80713600 & -0.03295800 \\ \mathrm{~N} & -4.42369700 & 1.21557100 & 1.22018300 \\ \mathrm{C} & -3.48889800 & 1.96356200 & 1.91723100 \\ \mathrm{~N} & -3.17134200 & 3.07182600 & 1.14342100 \\ \mathrm{~N} & -3.30614400 & 3.12467900 & -1.29732700 \\ \mathrm{~N} & -4.34885200 & 1.14894900 & -1.21707500 \\ \mathrm{C} & -4.97716400 & -0.00266900 & 1.75370300 \\ \mathrm{O} & -3.06388700 & 1.72189300 & 3.02150500 \\ \mathrm{C} & -4.91511300 & -0.07155500 & -1.73080900 \\ \mathrm{C} & -3.49191600 & 1.92693200 & -1.97388400 \\ \mathrm{O} & -3.02983500 & 1.64008400 & -3.05229400 \\ \mathrm{~N} & -4.34310400 & -1.27217000 & -1.17757700 \\ \mathrm{C} & -4.76597300 & -1.88102700 & 0.04066100 \\ \mathrm{C} & -3.51220200 & -2.08745700 & -1.92591400 \\ \mathrm{C} & -3.92935000 & -3.21815200 & 0.09161100 \\ \mathrm{~N} & -4.40322500 & -1.23008900 & 1.26446700 \\ \mathrm{~N} & -3.27763100 & -3.23709500 & -1.18560300 \\ \mathrm{O} & -3.10384200 & -1.86210900 & -3.03998500 \\ \mathrm{~N} & -3.10010900 & -3.04943900 & 1.24228900 \\ & & \end{array}$




\begin{tabular}{|c|c|c|c|}
\hline $\mathrm{C}$ & -3.41727700 & -1.91142400 & 1.96420100 \\
\hline $\mathrm{O}$ & -2.95755900 & -1.59211100 & 3.03452900 \\
\hline $\mathrm{C}$ & -2.47937600 & -4.32167200 & -1.69522100 \\
\hline $\mathrm{C}$ & -2.28877800 & -4.09474100 & 1.81424900 \\
\hline $\mathrm{N}$ & -0.98155500 & -4.21934500 & 1.21724600 \\
\hline $\mathrm{N}$ & -1.12382200 & -4.39039400 & -1.21387000 \\
\hline $\mathrm{C}$ & 0.16952600 & -3.98414400 & 1.95549000 \\
\hline C & -0.72167900 & -4.97246800 & 0.03195400 \\
\hline C & 0.84907000 & -4.96826100 & -0.06770900 \\
\hline $\mathrm{N}$ & 1.24847000 & -4.41806100 & 1.19356900 \\
\hline C & -0.04825000 & -3.92969700 & -1.96286200 \\
\hline $\mathrm{N}$ & 1.10542600 & -4.18577700 & -1.23459400 \\
\hline C & 2.60601800 & -4.33582700 & 1.66882800 \\
\hline C & 2.41149400 & -4.03941900 & -1.82860800 \\
\hline $\mathrm{O}$ & -0.10637900 & -3.44066600 & -3.06404800 \\
\hline $\mathrm{O}$ & 0.22532700 & -3.53059400 & 3.07178300 \\
\hline $\mathrm{N}$ & 3.37690600 & -3.22013600 & 1.18357500 \\
\hline $\mathrm{N}$ & 3.20188600 & -2.98393700 & -1.24383600 \\
\hline C & 3.57621000 & -2.07668900 & 1.94760200 \\
\hline C & 4.02897100 & -3.15603700 & -0.09140400 \\
\hline $\mathrm{C}$ & 4.82972800 & -1.80096000 & -0.00997600 \\
\hline $\mathrm{N}$ & 4.34492000 & -1.20318800 & 1.19234600 \\
\hline $\mathrm{O}$ & 3.18940200 & -1.89869400 & 3.07595000 \\
\hline C & 4.88568700 & 0.00300900 & 1.76718200 \\
\hline $\mathrm{N}$ & 4.49872500 & -1.15810900 & -1.24739900 \\
\hline C & 3.55199600 & -1.86208000 & -1.98201300 \\
\hline $\mathrm{O}$ & 3.14525400 & -1.57110400 & -3.07948300 \\
\hline C & 5.07093100 & 0.07617900 & -1.72287600 \\
\hline $\mathrm{N}$ & 4.30804700 & 1.20980600 & 1.22990100 \\
\hline $\mathrm{N}$ & 4.47774600 & 1.28245400 & -1.20362200 \\
\hline C & 3.48968100 & 2.02161900 & 1.99956200 \\
\hline $\mathrm{C}$ & 4.79427000 & 1.87376700 & 0.06354500 \\
\hline C & 3.97552900 & 3.22074200 & 0.03734300 \\
\hline $\mathrm{N}$ & 3.30540900 & 3.20945900 & 1.30061300 \\
\hline $\mathrm{O}$ & 3.05487700 & 1.76850900 & 3.09557400 \\
\hline $\mathrm{C}$ & 2.41770900 & 4.23825700 & 1.77453800 \\
\hline $\mathrm{N}$ & 3.17213600 & 3.10467300 & -1.14374100 \\
\hline $\mathrm{C}$ & 3.56285200 & 2.02706700 & -1.93399500 \\
\hline $\mathrm{O}$ & 3.20693200 & 1.80661300 & -3.06488300 \\
\hline C & 2.46362700 & 4.21599300 & -1.73783600 \\
\hline $\mathrm{N}$ & 1.10248600 & 4.23424900 & 1.17503400 \\
\hline $\mathrm{N}$ & 1.11724300 & 4.39823300 & -1.26246800 \\
\hline C & 0.77225700 & 4.97984200 & -0.00257800 \\
\hline C & 0.01484300 & 3.92825600 & -1.96294400 \\
\hline $\mathrm{C}$ & -0.80229100 & 4.95998500 & -0.02872900 \\
\hline $\mathrm{N}$ & -1.11426000 & 4.18229000 & -1.19030500 \\
\hline $\mathrm{C}$ & -0.02021800 & 3.96846000 & 1.95306500 \\
\hline $\mathrm{N}$ & -1.13287000 & 4.39554100 & 1.24285600 \\
\hline $\mathrm{O}$ & 0.02966500 & 3.43749800 & -3.06444700 \\
\hline $\mathrm{O}$ & -0.02292700 & 3.50025800 & 3.06460800 \\
\hline C & -2.42739900 & 4.15023700 & -1.79374600 \\
\hline $\mathrm{C}$ & -2.47374300 & 4.19397400 & 1.72593000 \\
\hline $\mathrm{H}$ & 2.95282000 & -4.98495200 & -1.74701200 \\
\hline $\mathrm{H}$ & 2.25650800 & -3.77912900 & -2.87753300 \\
\hline $\mathrm{H}$ & 2.55430400 & -4.23264100 & 2.75482600 \\
\hline $\mathrm{H}$ & 3.12322500 & -5.25896200 & 1.39298800 \\
\hline $\mathrm{H}$ & 5.96777500 & 0.02416500 & 1.61750100 \\
\hline $\mathrm{H}$ & 4.64880900 & -0.02004900 & 2.83284700 \\
\hline $\mathrm{H}$ & 6.13556200 & 0.08092100 & -1.47332200 \\
\hline $\mathrm{H}$ & 4.92850500 & 0.09637900 & -2.80545700 \\
\hline $\mathrm{H}$ & 2.88010200 & 5.21324600 & 1.60284800 \\
\hline
\end{tabular}




$\begin{array}{lrrr}\mathrm{H} & 2.27954200 & 4.06417400 & 2.84355700 \\ \mathrm{H} & 3.02803500 & 5.13361700 & -1.55552500 \\ \mathrm{H} & 2.39544700 & 4.01446700 & -2.80871100 \\ \mathrm{H} & -2.39367300 & 3.99996000 & 2.79732500 \\ \mathrm{H} & -3.05475700 & 5.10069000 & 1.54206800 \\ \mathrm{H} & -2.28158400 & 3.94976200 & -2.85706400 \\ \mathrm{H} & -2.90325400 & 5.12341900 & -1.65029100 \\ \mathrm{H} & -6.04860300 & -0.01549700 & 1.53538100 \\ \mathrm{H} & -4.80469500 & 0.02025900 & 2.83173500 \\ \mathrm{H} & -4.71161200 & -0.09111300 & -2.80322700 \\ \mathrm{H} & -5.99189100 & -0.06933200 & -1.54697400 \\ \mathrm{H} & -2.97912300 & -5.26248600 & -1.44836000 \\ \mathrm{H} & -2.42303400 & -4.18802600 & -2.77753300 \\ \mathrm{H} & -2.13182300 & -3.84106000 & 2.86443600 \\ \mathrm{H} & -2.81824800 & -5.04642900 & 1.72708100 \\ \mathrm{O} & -4.73035300 & -4.34638100 & 0.29176000 \\ \mathrm{O} & -6.14405800 & -2.10930800 & -0.04427500 \\ \mathrm{O} & -6.17260200 & 2.00309300 & -0.13537000 \\ \mathrm{O} & -4.80647900 & 4.28173800 & 0.09190700 \\ \mathrm{O} & -1.35161100 & 6.23526600 & -0.19850400 \\ \mathrm{O} & 1.29045900 & 6.27122900 & 0.14170900 \\ \mathrm{O} & 4.80050700 & 4.34291300 & -0.11064400 \\ \mathrm{O} & 6.16804100 & 2.08034200 & 0.21823300 \\ \mathrm{O} & 6.20849600 & -1.99917100 & 0.12591900 \\ \mathrm{O} & 4.86213100 & -4.25837200 & -0.31111700 \\ \mathrm{O} & 1.37640300 & -6.24859600 & -0.27268200 \\ \mathrm{O} & -1.24382100 & -6.25918900 & 0.20475800 \\ \mathrm{H} & -5.31429900 & -4.41698400 & -0.47272200 \\ \mathrm{H} & -6.41261800 & -2.52582200 & 0.78330300 \\ \mathrm{H} & -6.44191000 & 2.52052500 & 0.63287300 \\ \mathrm{H} & -5.30458800 & 4.36015800 & -0.72989600 \\ \mathrm{H} & -1.13973100 & 6.74052200 & 0.59497800 \\ \mathrm{H} & 1.06945800 & 6.75402300 & -0.66323900 \\ \mathrm{H} & 5.28088400 & 4.45040900 & 0.71850400 \\ \mathrm{H} & 6.46043400 & 2.60381200 & -0.53756200 \\ \mathrm{H} & 6.51490700 & -2.41206100 & -0.69034200 \\ \mathrm{H} & 5.43112100 & -4.33935000 & 0.46367600 \\ \mathrm{H} & 1.18253000 & -6.76236000 & 0.52044200 \\ \mathrm{H} & -1.02840400 & -6.75867400 & -0.59200700 \\ \mathrm{C} & -1.22473000 & -0.03415300 & -0.59242000 \\ \mathrm{H} & -1.26517100 & -0.07867100 & -1.66136700 \\ \mathrm{H} & -1.18205000 & 0.01920600 & 0.61156700 \\ & -1.15554400 & 0.06320100 & 1.68087300\end{array}$

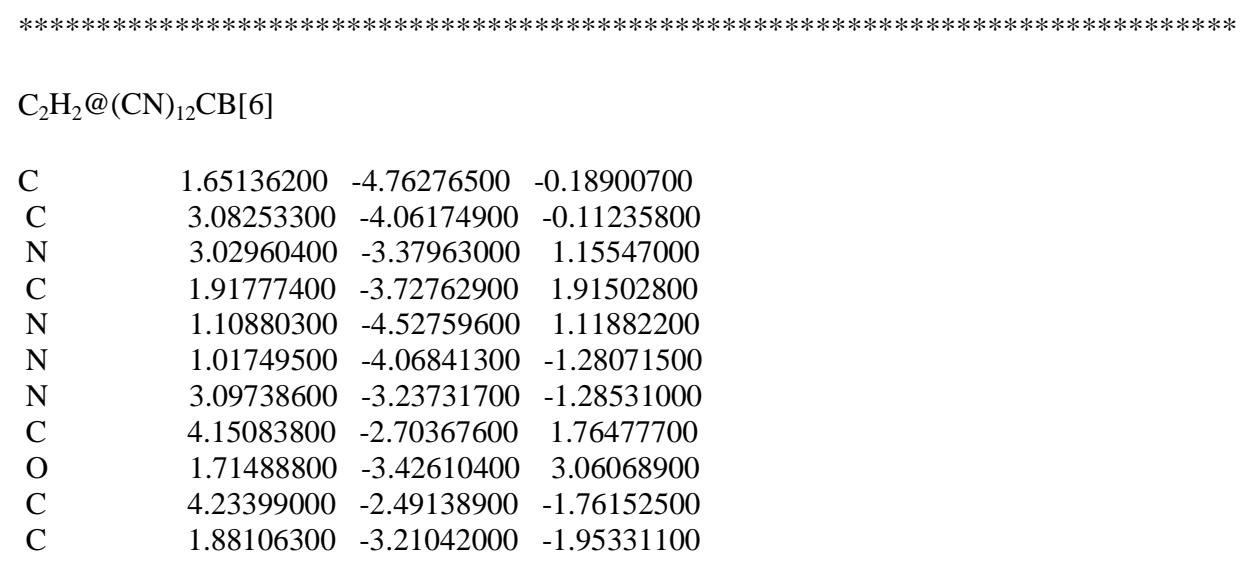




\begin{tabular}{|c|c|c|c|}
\hline $\mathrm{O}$ & 1.63495400 & -2.59372000 & -2.95583500 \\
\hline $\mathrm{N}$ & 4.40151400 & -1.19808700 & -1.13975000 \\
\hline $\mathrm{C}$ & 5.07297800 & -0.98230000 & 0.11756400 \\
\hline $\mathrm{C}$ & 4.28698300 & -0.03733700 & -1.89807300 \\
\hline C & 5.11645900 & 0.60911100 & 0.21299200 \\
\hline $\mathrm{N}$ & 4.35643500 & -1.35438300 & 1.30220400 \\
\hline $\mathrm{N}$ & 4.69801100 & 1.02065000 & -1.09667500 \\
\hline $\mathrm{O}$ & 3.93518200 & 0.03195400 & -3.04488900 \\
\hline $\mathrm{N}$ & 4.20563800 & 0.87951800 & 1.29679500 \\
\hline C & 3.81075000 & -0.26976600 & 1.97337600 \\
\hline $\mathrm{O}$ & 3.15184300 & -0.31366200 & 2.97826400 \\
\hline C & 4.67013400 & 2.38120000 & -1.57709000 \\
\hline C & 4.03777900 & 2.17441300 & 1.90975100 \\
\hline $\mathrm{N}$ & 3.07504200 & 3.01614800 & 1.24151600 \\
\hline $\mathrm{N}$ & 3.52424300 & 3.15588000 & -1.16245800 \\
\hline C & 1.95149600 & 3.46565100 & 1.93025300 \\
\hline C & 3.38302200 & 3.83406500 & 0.09246800 \\
\hline C & 2.03192600 & 4.64920200 & -0.09568000 \\
\hline $\mathrm{N}$ & 1.36973300 & 4.46654000 & 1.16254500 \\
\hline $\mathrm{C}$ & 2.36316500 & 3.19909000 & -1.92486700 \\
\hline $\mathrm{N}$ & 1.44463000 & 3.98704300 & -1.23651000 \\
\hline $\mathrm{C}$ & 0.17127400 & 5.14652100 & 1.59265600 \\
\hline C & 0.25587100 & 4.45107600 & -1.90998800 \\
\hline $\mathrm{O}$ & 2.19632800 & 2.68380200 & -2.99788200 \\
\hline $\mathrm{O}$ & 1.57669000 & 3.08568500 & 3.00723800 \\
\hline $\mathrm{N}$ & -1.06464300 & 4.58126400 & 1.10851900 \\
\hline $\mathrm{N}$ & -0.97574500 & 4.04662600 & -1.27664700 \\
\hline $\mathrm{C}$ & -1.85076700 & 3.76396200 & 1.91148400 \\
\hline C & -1.60869000 & 4.77532600 & -0.20476000 \\
\hline $\mathrm{C}$ & -3.03631600 & 4.07269400 & -0.10637800 \\
\hline $\mathrm{N}$ & -2.95681600 & 3.38669200 & 1.15769500 \\
\hline $\mathrm{O}$ & -1.63691600 & 3.47307000 & 3.05772600 \\
\hline C & -4.06240100 & 2.69571800 & 1.77776300 \\
\hline $\mathrm{N}$ & -3.06887500 & 3.25051100 & -1.28007300 \\
\hline C & -1.84687600 & 3.18266700 & -1.93102200 \\
\hline $\mathrm{O}$ & -1.59963500 & 2.53147500 & -2.91281400 \\
\hline C & -4.21684000 & 2.51802700 & -1.75259200 \\
\hline $\mathrm{N}$ & -4.27551100 & 1.35453000 & 1.29578600 \\
\hline $\mathrm{N}$ & -4.40211700 & 1.22504200 & -1.14186900 \\
\hline $\mathrm{C}$ & -3.75836800 & 0.25685500 & 1.96394000 \\
\hline $\mathrm{C}$ & -5.03483400 & 1.00044000 & 0.13051200 \\
\hline C & -5.09170600 & -0.59441000 & 0.21123200 \\
\hline $\mathrm{N}$ & -4.22644300 & -0.88053200 & 1.32379800 \\
\hline $\mathrm{O}$ & -3.06525000 & 0.28430100 & 2.94856300 \\
\hline $\mathrm{C}$ & -4.04832100 & -2.18322700 & 1.91143800 \\
\hline $\mathrm{N}$ & -4.63002700 & -0.99843300 & -1.08712100 \\
\hline $\mathrm{C}$ & -4.21940600 & 0.06351000 & -1.87804300 \\
\hline $\mathrm{O}$ & -3.82071600 & -0.00330000 & -3.01157200 \\
\hline C & -4.60003000 & -2.35313300 & -1.58243900 \\
\hline $\mathrm{N}$ & -3.06878200 & -3.00981800 & 1.24830700 \\
\hline $\mathrm{N}$ & -3.46643200 & -3.13993300 & -1.16254800 \\
\hline C & -3.34761700 & -3.82360100 & 0.09092800 \\
\hline C & -2.30078000 & -3.19221400 & -1.91445500 \\
\hline C & -1.99004900 & -4.63716800 & -0.07664900 \\
\hline $\mathrm{N}$ & -1.40508000 & -4.00963200 & -1.23574900 \\
\hline $\mathrm{C}$ & -1.91422500 & -3.39822600 & 1.91626300 \\
\hline $\mathrm{N}$ & -1.32497200 & -4.41172100 & 1.17394400 \\
\hline $\mathrm{O}$ & -2.11748900 & -2.66686800 & -2.98123200 \\
\hline $\mathrm{O}$ & -1.51933000 & -2.96236400 & 2.96639500 \\
\hline C & -0.21689000 & -4.48679400 & -1.89953700 \\
\hline C & -0.12572100 & -5.07816800 & 1.62137800 \\
\hline
\end{tabular}




\begin{tabular}{|c|c|c|c|}
\hline $\mathrm{H}$ & 0.28009600 & 5.54378200 & -2.00882900 \\
\hline $\mathrm{H}$ & 0.26782400 & 3.99449900 & -2.90270100 \\
\hline $\mathrm{H}$ & 0.13716100 & 5.06707700 & 2.68100000 \\
\hline $\mathrm{H}$ & 0.23375300 & 6.19945500 & 1.30012600 \\
\hline $\mathrm{H}$ & -4.98738900 & 3.27012200 & 1.65281200 \\
\hline $\mathrm{H}$ & -3.81742500 & 2.62084800 & 2.83952200 \\
\hline $\mathrm{H}$ & -5.11331400 & 3.13175100 & -1.61203500 \\
\hline $\mathrm{H}$ & -4.06215000 & 2.33177600 & -2.81734600 \\
\hline $\mathrm{H}$ & -5.01327400 & -2.70334900 & 1.94705400 \\
\hline $\mathrm{H}$ & -3.68051500 & -2.02341800 & 2.92773100 \\
\hline $\mathrm{H}$ & -5.52042500 & -2.86973700 & -1.29239200 \\
\hline $\mathrm{H}$ & -4.54597500 & -2.27986300 & -2.67022300 \\
\hline $\mathrm{H}$ & -0.08729500 & -4.96770400 & 2.70687300 \\
\hline $\mathrm{H}$ & -0.19044500 & -6.13931800 & 1.35993500 \\
\hline $\mathrm{H}$ & -0.22956300 & -4.05249400 & -2.90213100 \\
\hline $\mathrm{H}$ & -0.24219500 & -5.58125800 & -1.97325400 \\
\hline $\mathrm{H}$ & 5.07082800 & -3.27768600 & 1.60803700 \\
\hline $\mathrm{H}$ & 3.93098100 & -2.64645800 & 2.83297100 \\
\hline $\mathrm{H}$ & 4.07051900 & -2.29987800 & -2.82430500 \\
\hline $\mathrm{H}$ & 5.13976800 & -3.09418800 & -1.63083600 \\
\hline $\mathrm{H}$ & 5.58272800 & 2.89922600 & -1.26650500 \\
\hline $\mathrm{H}$ & 4.63324600 & 2.32016200 & -2.66639100 \\
\hline $\mathrm{H}$ & 3.64945900 & 2.00117500 & 2.91656900 \\
\hline $\mathrm{H}$ & 5.00749200 & 2.68395900 & 1.97286800 \\
\hline C & -1.10941500 & -0.25625300 & -0.89796800 \\
\hline $\mathrm{H}$ & -1.18042900 & -0.13593500 & -1.95963400 \\
\hline C & -1.03551600 & -0.39593000 & 0.29805500 \\
\hline $\mathrm{H}$ & -0.96775400 & -0.50722500 & 1.36069700 \\
\hline C & -1.67270000 & 6.21983100 & -0.52487300 \\
\hline $\mathrm{C}$ & -4.17716400 & 5.01731300 & -0.10699400 \\
\hline C & -6.36239700 & 1.65600400 & 0.16435200 \\
\hline $\mathrm{C}$ & -6.44579000 & -1.14071000 & 0.46049600 \\
\hline C & -4.53644300 & -4.67244300 & 0.33258400 \\
\hline C & -2.18087500 & -6.08644300 & -0.30940500 \\
\hline C & 1.71166500 & -6.21724400 & -0.46325700 \\
\hline C & 4.22154300 & -5.00872000 & -0.13584500 \\
\hline C & 6.40820100 & -1.62332800 & 0.10203000 \\
\hline $\mathrm{C}$ & 6.45878900 & 1.15789800 & 0.51442800 \\
\hline C & 4.56800500 & 4.67974700 & 0.36179200 \\
\hline $\mathrm{C}$ & 2.23101600 & 6.08942200 & -0.37397000 \\
\hline $\mathrm{N}$ & -5.11491100 & 5.69391700 & -0.08304300 \\
\hline $\mathrm{N}$ & -7.36245700 & 2.23701300 & 0.17152200 \\
\hline $\mathrm{N}$ & -7.47035800 & -1.63917200 & 0.65928800 \\
\hline $\mathrm{N}$ & -5.50296600 & -5.27079000 & 0.54570600 \\
\hline $\mathrm{N}$ & -2.25880100 & -7.22188800 & -0.51491000 \\
\hline $\mathrm{N}$ & 1.68414400 & -7.35274500 & -0.68141000 \\
\hline $\mathrm{N}$ & 5.15924600 & -5.68586100 & -0.13351600 \\
\hline $\mathrm{N}$ & 7.41394800 & -2.19338100 & 0.06668900 \\
\hline $\mathrm{N}$ & 7.47351700 & 1.65727300 & 0.75674200 \\
\hline $\mathrm{N}$ & 5.53039400 & 5.27525000 & 0.60011000 \\
\hline $\mathrm{N}$ & 2.31433500 & 7.21694800 & -0.61749900 \\
\hline $\mathrm{N}$ & -1.64932600 & 7.34738800 & -0.78130600 \\
\hline
\end{tabular}

$\mathrm{CO}_{2} @\left(\mathrm{CH}_{3}\right)_{12} \mathrm{CB}[6]$

$\begin{array}{lllll}\text { C } & -3.92532200 & 3.24571900 & -0.37081500\end{array}$

$\begin{array}{llll}\text { C } & -4.77367800 & 1.91522600 & -0.20919400\end{array}$ 


\begin{tabular}{|c|c|c|c|}
\hline $\mathrm{N}$ & -4.32355300 & 1.42919400 & 1.08923100 \\
\hline $\mathrm{C}$ & -3.72464900 & 2.42894100 & 1.83516100 \\
\hline $\mathrm{N}$ & -3.48872200 & 3.49797200 & 0.98312500 \\
\hline $\mathrm{N}$ & -2.86916600 & 2.82356900 & -1.28643800 \\
\hline $\mathrm{N}$ & -4.33239000 & 1.12841600 & -1.33160700 \\
\hline C & -4.85036800 & 0.27454300 & 1.77580200 \\
\hline $\mathrm{O}$ & -3.50979600 & 2.40655400 & 3.02506200 \\
\hline C & -4.87742800 & -0.12952600 & -1.76673100 \\
\hline $\mathrm{C}$ & -3.18487600 & 1.62767100 & -1.92014000 \\
\hline $\mathrm{O}$ & -2.60662200 & 1.14350700 & -2.86465300 \\
\hline $\mathrm{N}$ & -4.37634000 & -1.29733900 & -1.08568700 \\
\hline C & -4.81546900 & -1.77483800 & 0.21768800 \\
\hline C & -3.75462200 & -2.28572200 & -1.82540500 \\
\hline $\mathrm{C}$ & -3.99275700 & -3.12710100 & 0.37074900 \\
\hline $\mathrm{N}$ & -4.34168000 & -0.99494700 & 1.33392700 \\
\hline $\mathrm{N}$ & -3.52573100 & -3.35846600 & -0.97747300 \\
\hline $\mathrm{O}$ & -3.52346400 & -2.25614800 & -3.01208200 \\
\hline $\mathrm{N}$ & -2.95605200 & -2.74814500 & 1.32084800 \\
\hline $\mathrm{C}$ & -3.20893400 & -1.52613500 & 1.92052900 \\
\hline $\mathrm{O}$ & -2.59527600 & -1.04132800 & 2.84381000 \\
\hline C & -2.77121400 & -4.49167700 & -1.44707500 \\
\hline C & -2.05172200 & -3.65298200 & 1.98007300 \\
\hline $\mathrm{N}$ & -0.86280100 & -3.98376000 & 1.23124900 \\
\hline $\mathrm{N}$ & -1.37257700 & -4.53313100 & -1.09314600 \\
\hline C & 0.36176500 & -3.89686800 & 1.88225500 \\
\hline C & -0.81187800 & -4.97499000 & 0.15813300 \\
\hline C & 0.72957600 & -4.98764300 & -0.15631800 \\
\hline $\mathrm{N}$ & 1.29731400 & -4.55093000 & 1.09354300 \\
\hline C & -0.42656200 & -3.89636200 & -1.88376900 \\
\hline $\mathrm{N}$ & 0.79618100 & -4.00060100 & -1.23232400 \\
\hline C & 2.69619000 & -4.53182500 & 1.44829600 \\
\hline C & 1.99045000 & -3.68940700 & -1.98097200 \\
\hline $\mathrm{O}$ & -0.62236100 & -3.39440200 & -2.96531400 \\
\hline $\mathrm{O}$ & 0.56579500 & -3.39435900 & 2.96202700 \\
\hline $\mathrm{N}$ & 3.46969500 & -3.41231000 & 0.97661800 \\
\hline $\mathrm{N}$ & 2.90839800 & -2.79774000 & -1.32282500 \\
\hline C & 3.72201000 & -2.34459700 & 1.82417800 \\
\hline $\mathrm{C}$ & 3.93945500 & -3.19105800 & -0.37253100 \\
\hline C & 4.78455700 & -1.85190400 & -0.22315000 \\
\hline $\mathrm{N}$ & 4.36074700 & -1.36837300 & 1.08286100 \\
\hline $\mathrm{O}$ & 3.49521500 & -2.31082900 & 3.01158600 \\
\hline C & 4.88297700 & -0.20841400 & 1.76117100 \\
\hline $\mathrm{N}$ & 4.31705800 & -1.06303300 & -1.33587700 \\
\hline C & 3.17603600 & -1.57799300 & -1.92067600 \\
\hline $\mathrm{O}$ & 2.56665000 & -1.08325800 & -2.84155200 \\
\hline $\mathrm{C}$ & 4.84557400 & 0.19718500 & -1.78101500 \\
\hline $\mathrm{N}$ & 4.35585800 & 1.05794300 & 1.32788000 \\
\hline $\mathrm{N}$ & 4.34205500 & 1.36104800 & -1.09262300 \\
\hline C & 3.21590300 & 1.57278800 & 1.91770600 \\
\hline C & 4.80528600 & 1.83842000 & 0.20438600 \\
\hline C & 3.97881200 & 3.18192100 & 0.37033500 \\
\hline $\mathrm{N}$ & 2.91645000 & 2.77350300 & 1.28518600 \\
\hline $\mathrm{O}$ & 2.63153000 & 1.09592000 & 2.86218300 \\
\hline C & 2.03128700 & 3.68236300 & 1.97401500 \\
\hline $\mathrm{N}$ & 3.54590700 & 3.44489200 & -0.98276800 \\
\hline $\mathrm{C}$ & 3.76034000 & 2.37245200 & -1.83646300 \\
\hline $\mathrm{O}$ & 3.54283200 & 2.35513400 & -3.02593800 \\
\hline $\mathrm{C}$ & 2.78056600 & 4.58009200 & -1.43397000 \\
\hline $\mathrm{N}$ & 0.85111000 & 4.05909900 & 1.23901600 \\
\hline $\mathrm{N}$ & 1.38212100 & 4.60500700 & -1.07936900 \\
\hline C & 0.81122900 & 5.04977500 & 0.16693500 \\
\hline
\end{tabular}




\begin{tabular}{|c|c|c|c|}
\hline $\mathrm{C}$ & 0.44316300 & 3.95332200 & -1.86855800 \\
\hline C & -0.72860000 & 5.06284200 & -0.16179600 \\
\hline $\mathrm{N}$ & -0.78446000 & 4.07730000 & -1.23780000 \\
\hline C & -0.37869000 & 3.95148600 & 1.86871700 \\
\hline $\mathrm{N}$ & -1.30660500 & 4.62242900 & 1.08271200 \\
\hline $\mathrm{O}$ & 0.65407500 & 3.42782400 & -2.93650100 \\
\hline $\mathrm{O}$ & -0.59867400 & 3.42376900 & 2.93373600 \\
\hline C & -1.97072700 & 3.72080300 & -1.97312100 \\
\hline C & -2.70513200 & 4.61918700 & 1.43772300 \\
\hline $\mathrm{H}$ & 2.50776200 & -4.60847000 & -2.28237000 \\
\hline $\mathrm{H}$ & 1.64864800 & -3.17415200 & -2.88267000 \\
\hline $\mathrm{H}$ & 2.73963500 & -4.47520300 & 2.53824500 \\
\hline $\mathrm{H}$ & 3.15460200 & -5.46723800 & 1.11654500 \\
\hline $\mathrm{H}$ & 5.97504700 & -0.17317200 & 1.69465000 \\
\hline $\mathrm{H}$ & 4.59547100 & -0.33445400 & 2.80762700 \\
\hline $\mathrm{H}$ & 5.93867700 & 0.15465800 & -1.73647700 \\
\hline $\mathrm{H}$ & 4.53957300 & 0.32537600 & -2.82193500 \\
\hline $\mathrm{H}$ & 2.58187400 & 4.57251900 & 2.30296600 \\
\hline $\mathrm{H}$ & 1.67276900 & 3.15389500 & 2.86137700 \\
\hline $\mathrm{H}$ & 3.24717500 & 5.50381400 & -1.08194800 \\
\hline $\mathrm{H}$ & 2.82694500 & 4.54526900 & -2.52458500 \\
\hline $\mathrm{H}$ & -2.75204800 & 4.58115600 & 2.52821800 \\
\hline $\mathrm{H}$ & -3.15678100 & 5.55159900 & 1.08906500 \\
\hline $\mathrm{H}$ & -1.62060200 & 3.18968400 & -2.86228100 \\
\hline $\mathrm{H}$ & -2.50804900 & 4.62010700 & -2.29893200 \\
\hline $\mathrm{H}$ & -5.94381400 & 0.25118600 & 1.72626500 \\
\hline $\mathrm{H}$ & -4.54686500 & 0.39797500 & 2.81804400 \\
\hline $\mathrm{H}$ & -4.58739400 & -0.26036800 & -2.81189200 \\
\hline $\mathrm{H}$ & -5.96910600 & -0.07719300 & -1.70506500 \\
\hline $\mathrm{H}$ & -3.24482600 & -5.41849500 & -1.11263000 \\
\hline $\mathrm{H}$ & -2.81464500 & -4.43678100 & -2.53712100 \\
\hline $\mathrm{H}$ & -1.70191900 & -3.14141500 & 2.88077600 \\
\hline $\mathrm{H}$ & -2.58263900 & -4.56367800 & 2.28337800 \\
\hline $\mathrm{H}$ & -5.22304800 & -4.08553100 & 1.87559300 \\
\hline $\mathrm{H}$ & -6.84014300 & -0.99289400 & 0.09473600 \\
\hline $\mathrm{H}$ & -6.57830300 & 2.79677900 & 0.59558600 \\
\hline $\mathrm{H}$ & -4.09997300 & 5.31879200 & -1.01528100 \\
\hline $\mathrm{H}$ & -0.68175400 & 6.76852600 & -1.47732200 \\
\hline $\mathrm{H}$ & 2.40500800 & 6.31128100 & 0.94305400 \\
\hline $\mathrm{H}$ & 5.63024100 & 4.58552400 & 0.27435100 \\
\hline $\mathrm{H}$ & 6.83354600 & 1.07160800 & 0.04126900 \\
\hline $\mathrm{H}$ & 6.62256200 & -2.45733000 & -1.22055900 \\
\hline $\mathrm{H}$ & 4.11995900 & -5.27648600 & -0.96799900 \\
\hline $\mathrm{H}$ & 0.70376100 & -6.68992700 & -1.47975300 \\
\hline $\mathrm{H}$ & -2.41633500 & -6.24651800 & 0.89852000 \\
\hline $\mathrm{C}$ & -1.36648700 & -6.32361800 & 0.61150600 \\
\hline C & 1.26259400 & -6.34618600 & -0.60580900 \\
\hline C & 4.74504800 & -4.38595500 & -0.88480200 \\
\hline C & 6.30093000 & -2.04996100 & -0.26076300 \\
\hline C & 6.32370600 & 2.02315300 & 0.20393900 \\
\hline C & 4.79385100 & 4.34723500 & 0.93264900 \\
\hline $\mathrm{C}$ & 1.36355200 & 6.39627900 & 0.62815700 \\
\hline C & -1.25912000 & 6.41989600 & -0.61754900 \\
\hline C & -4.72107400 & 4.42594700 & -0.92984100 \\
\hline C & -6.28903500 & 2.12387800 & -0.21496800 \\
\hline $\mathrm{C}$ & -6.33493700 & -1.94885900 & 0.24619400 \\
\hline $\mathrm{C}$ & -4.81833600 & -4.30716900 & 0.88535600 \\
\hline $\mathrm{H}$ & -1.29489600 & -7.06555900 & -0.18543000 \\
\hline $\mathrm{H}$ & -0.81319000 & -6.67394500 & 1.48634700 \\
\hline $\mathrm{H}$ & -5.63461500 & -4.53662800 & 0.19910800 \\
\hline $\mathrm{H}$ & -4.20766100 & -5.20703800 & 0.97475800 \\
\hline
\end{tabular}




$\begin{array}{lrrr}\mathrm{H} & -6.65173400 & -2.61434700 & -0.55995400 \\ \mathrm{H} & -6.66909000 & -2.35012800 & 1.20428500 \\ \mathrm{H} & -6.62752500 & 2.53329400 & -1.16804600 \\ \mathrm{H} & -6.81402900 & 1.17989900 & -0.05656000 \\ \mathrm{H} & -5.10010500 & 4.19065900 & -1.92737900 \\ \mathrm{H} & -5.55470500 & 4.67486000 & -0.27194800 \\ \mathrm{H} & -2.30221700 & 6.35338400 & -0.93153300 \\ \mathrm{H} & -1.19907600 & 7.15769900 & 0.18437600 \\ \mathrm{H} & 1.31679600 & 7.13785300 & -0.17115400 \\ \mathrm{H} & 0.79106600 & 6.75132000 & 1.48855400 \\ \mathrm{H} & 4.18727500 & 5.24955300 & 1.02288400 \\ \mathrm{H} & 5.17069400 & 4.10184400 & 1.92859200 \\ \mathrm{H} & 6.61962100 & 2.69326900 & -0.60654700 \\ \mathrm{H} & 6.67296400 & 2.42537700 & 1.15622300 \\ \mathrm{H} & 6.82186800 & -1.10184800 & -0.11355500 \\ \mathrm{H} & 6.61245100 & -2.71942000 & 0.54410500 \\ \mathrm{H} & 5.15016700 & -4.17511900 & -1.87721900 \\ \mathrm{H} & 5.55974900 & -4.62532900 & -0.20004600 \\ \mathrm{H} & 2.31353900 & -6.28678800 & -0.89306800 \\ \mathrm{H} & 1.17894000 & -7.08471600 & 0.19313300 \\ \mathrm{C} & -0.00190900 & -0.87635200 & 0.01452400 \\ \mathrm{O} & 1.10658300 & -0.89771100 & 0.37148600 \\ \mathrm{O} & -1.11011600 & -0.85387700 & -0.34305800\end{array}$

$\begin{array}{lrrr}\text { * } * * * * * * * * * * * * * * * * * * * * * * * * * * * * * * * * * * * * * * * * * * * * * * * * * * * * * * * * * * * * * * * * * * * * * * * * \\ \mathrm{CO}_{2} @\left(\mathrm{C}_{2} \mathrm{H}_{5}\right)_{12} \mathrm{CB}[6] & & \\ \mathrm{C} & & & \\ \mathrm{C} & -0.29665100 & -4.99519400 & -0.51256500 \\ \mathrm{~N} & 1.26793100 & -4.96249900 & -0.23009600 \\ \mathrm{C} & 1.29311600 & -4.44336100 & 1.13691400 \\ \mathrm{~N} & 0.11789300 & -4.74514500 & 1.79325700 \\ \mathrm{~N} & -0.82029300 & -5.11921600 & 0.83046500 \\ \mathrm{~N} & -0.53402600 & -3.69426800 & -1.15106100 \\ \mathrm{C} & 1.70388200 & -4.01510800 & -1.22006700 \\ \mathrm{O} & 2.41529100 & -3.97313900 & 1.92711400 \\ \mathrm{C} & -0.05079600 & -4.75330200 & 2.99150600 \\ \mathrm{C} & 3.05436000 & -3.72988800 & -1.62701800 \\ \mathrm{O} & 0.66701100 & -3.20124800 & -1.66058200 \\ \mathrm{~N} & 0.79695500 & -2.26203500 & -2.41262500 \\ \mathrm{C} & 3.71337100 & -2.67467200 & -0.89694800 \\ \mathrm{C} & 4.17997000 & -2.78074100 & 0.47426100 \\ \mathrm{C} & 4.39188500 & -1.69421400 & -1.58393000 \\ \mathrm{~N} & 4.82237500 & -1.33729800 & 0.71763500 \\ \mathrm{~N} & 3.10788900 & -2.81119000 & 1.43452400 \\ \mathrm{O} & 5.06202400 & -0.90354500 & -0.64640900 \\ \mathrm{~N} & 4.46200400 & -1.57737400 & -2.78635900 \\ \mathrm{C} & 3.72095400 & -0.64440700 & 1.39371900 \\ \mathrm{O} & 2.78260600 & -1.54444800 & 1.89266600 \\ \mathrm{C} & 1.87860300 & -1.28749400 & 2.65444400 \\ \mathrm{C} & 5.50543200 & 0.39892500 & -1.09139200 \\ \mathrm{~N} & 3.82909800 & 0.62230600 & 2.09214400 \\ \mathrm{~N} & 3.63426800 & 1.81422900 & 1.30386900 \\ \mathrm{C} & 4.62659100 & 1.52618500 & -0.88837300 \\ \mathrm{C} & 2.82263200 & 2.81615200 & 1.80129500 \\ \mathrm{C} & 4.54487200 & 2.34408100 & 0.29001800 \\ \mathrm{~N} & 3.69925800 & 3.56977900 & -0.22331000 \\ \mathrm{C} & 2.96738500 & 3.92492100 & 0.96410400 \\ & 3.52684700 & 1.76296500 & -1.71496700 \\ & & \end{array}$




\begin{tabular}{|c|c|c|c|}
\hline $\mathrm{N}$ & 2.88726500 & 2.89344200 & -1.23348500 \\
\hline $\mathrm{C}$ & 2.21536700 & 5.13292700 & 1.20403100 \\
\hline $\mathrm{C}$ & 1.72798700 & 3.38545200 & -1.93256800 \\
\hline $\mathrm{O}$ & 3.22638700 & 1.13186700 & -2.70038800 \\
\hline $\mathrm{O}$ & 2.15467900 & 2.76932700 & 2.80750500 \\
\hline $\mathrm{N}$ & 0.82012200 & 5.12148400 & 0.82917700 \\
\hline $\mathrm{N}$ & 0.53390100 & 3.69061400 & -1.14810900 \\
\hline C & -0.11791300 & 4.74785500 & 1.79254700 \\
\hline $\mathrm{C}$ & 0.29651400 & 4.99392500 & -0.51368000 \\
\hline C & -1.26728700 & 4.96238800 & -0.23108600 \\
\hline $\mathrm{N}$ & -1.29273600 & 4.44432400 & 1.13649100 \\
\hline $\mathrm{O}$ & 0.05085900 & 4.75758000 & 2.99077400 \\
\hline C & -2.41443600 & 3.97242200 & 1.92659700 \\
\hline $\mathrm{N}$ & -1.70349800 & 4.01460900 & -1.22023200 \\
\hline C & -0.66791000 & 3.19684600 & -1.65571900 \\
\hline $\mathrm{O}$ & -0.79906900 & 2.25413500 & -2.40326900 \\
\hline C & -3.05407500 & 3.73111100 & -1.62778900 \\
\hline $\mathrm{N}$ & -3.10652600 & 2.81072400 & 1.43263500 \\
\hline $\mathrm{N}$ & -3.71396600 & 2.67614200 & -0.89845400 \\
\hline C & -2.78447700 & 1.54420100 & 1.89383400 \\
\hline C & -4.17941800 & 2.78126800 & 0.47308300 \\
\hline C & -4.82269300 & 1.33788300 & 0.71594100 \\
\hline $\mathrm{N}$ & -3.72272200 & 0.64470600 & 1.39385400 \\
\hline $\mathrm{O}$ & -1.88294700 & 1.28736200 & 2.65856300 \\
\hline C & -3.83230800 & -0.62202700 & 2.09200000 \\
\hline $\mathrm{N}$ & -5.06083500 & 0.90379800 & -0.64837000 \\
\hline C & -4.39087300 & 1.69487100 & -1.58558500 \\
\hline $\mathrm{O}$ & -4.45993700 & 1.57759000 & -2.78806400 \\
\hline C & -5.50354700 & -0.39871800 & -1.09389400 \\
\hline $\mathrm{N}$ & -3.63641900 & -1.81411100 & 1.30405500 \\
\hline $\mathrm{N}$ & -4.62478100 & -1.52588900 & -0.88990400 \\
\hline C & -4.54531100 & -2.34379900 & 0.28862500 \\
\hline C & -3.52434900 & -1.76332900 & -1.71520400 \\
\hline $\mathrm{C}$ & -3.69929900 & -3.56983700 & -0.22316300 \\
\hline $\mathrm{N}$ & -2.88586200 & -2.89404100 & -1.23253200 \\
\hline $\mathrm{C}$ & -2.82597900 & -2.81618400 & 1.80305200 \\
\hline $\mathrm{N}$ & -2.96894000 & -3.92482400 & 0.96529000 \\
\hline $\mathrm{O}$ & -3.22241500 & -1.13276500 & -2.70053600 \\
\hline $\mathrm{O}$ & -2.16046400 & -2.76986600 & 2.81088600 \\
\hline C & -1.72981300 & -3.39111700 & -1.93304000 \\
\hline C & -2.21547500 & -5.13183800 & 1.20562900 \\
\hline $\mathrm{H}$ & 2.00113500 & 4.25771900 & -2.53028500 \\
\hline $\mathrm{H}$ & 1.44438100 & 2.58160800 & -2.61673100 \\
\hline $\mathrm{H}$ & 2.21283800 & 5.30988500 & 2.28148500 \\
\hline $\mathrm{H}$ & 2.72910700 & 5.95796400 & 0.70775600 \\
\hline $\mathrm{H}$ & -3.14634000 & 4.76245100 & 2.10449400 \\
\hline $\mathrm{H}$ & -1.97062400 & 3.70043100 & 2.88676000 \\
\hline $\mathrm{H}$ & -3.61376400 & 4.67012100 & -1.57824800 \\
\hline $\mathrm{H}$ & -3.03520500 & 3.38680500 & -2.66385800 \\
\hline $\mathrm{H}$ & -4.78686300 & -0.66031800 & 2.62539400 \\
\hline $\mathrm{H}$ & -3.03039700 & -0.63606600 & 2.83442500 \\
\hline $\mathrm{H}$ & -6.46074000 & -0.63008700 & -0.63192000 \\
\hline $\mathrm{H}$ & -5.64199800 & -0.28819300 & -2.17136400 \\
\hline $\mathrm{H}$ & -2.21257200 & -5.30787200 & 2.28323000 \\
\hline $\mathrm{H}$ & -2.72829400 & -5.95785900 & 0.71010200 \\
\hline $\mathrm{H}$ & -1.44718100 & -2.59184900 & -2.62288900 \\
\hline $\mathrm{H}$ & -2.00634100 & -4.26666900 & -2.52394700 \\
\hline $\mathrm{H}$ & 3.14699300 & -4.76368400 & 2.10356800 \\
\hline $\mathrm{H}$ & 1.97198500 & -3.70233200 & 2.88783800 \\
\hline $\mathrm{H}$ & 3.03486200 & -3.38464400 & -2.66277100 \\
\hline $\mathrm{H}$ & 3.61524900 & -4.66817100 & -1.57842600 \\
\hline
\end{tabular}




\begin{tabular}{|c|c|c|c|}
\hline $\mathrm{H}$ & 6.46221900 & 0.63002900 & -0.62840600 \\
\hline $\mathrm{H}$ & 5.64489200 & 0.28838800 & -2.16873700 \\
\hline $\mathrm{H}$ & 3.02608300 & 0.63612600 & 2.83337600 \\
\hline $\mathrm{H}$ & 4.78281600 & 0.66066300 & 2.62698200 \\
\hline 11 & 5.74196100 & -1.91638600 & 2.54557500 \\
\hline $\mathrm{H}$ & 4.51777200 & -4.87845500 & 0.59895000 \\
\hline $\mathrm{H}$ & 1.12835100 & -6.99802300 & 0.27999900 \\
\hline $\mathrm{H}$ & -1.85475800 & -6.11704100 & -1.43297300 \\
\hline $\mathrm{H}$ & -5.36742700 & -4.06386500 & -1.39197500 \\
\hline $\mathrm{H}$ & -6.14486300 & -1.82718200 & 1.56410700 \\
\hline $\mathrm{H}$ & -6.68895400 & 2.27443700 & 1.11491100 \\
\hline $\mathrm{H}$ & -4.51592700 & 4.87917800 & 0.60061200 \\
\hline $\mathrm{H}$ & -1.73039200 & 6.74303700 & -1.35226200 \\
\hline $\mathrm{H}$ & 1.85915800 & 6.09876900 & -1.43773400 \\
\hline $\mathrm{H}$ & 5.36934700 & 4.06279800 & -1.38932300 \\
\hline $\mathrm{H}$ & 6.14200300 & 1.82897200 & 1.56928600 \\
\hline C & 5.89223500 & 2.68814700 & 0.95535500 \\
\hline C & 4.66129900 & 4.63825400 & -0.78850100 \\
\hline C & 0.77451400 & 6.14687600 & -1.41419700 \\
\hline C & -1.82741700 & 6.40691000 & -0.32037200 \\
\hline $\mathrm{C}$ & -5.12713700 & 3.98308000 & 0.62272200 \\
\hline C & -6.09690700 & 1.49791900 & 1.59280100 \\
\hline C & -5.89414900 & -2.68696200 & 0.95142600 \\
\hline C & -4.66080400 & -4.63860700 & -0.78874200 \\
\hline C & -0.76946800 & -6.15270500 & -1.40969200 \\
\hline C & 1.82907600 & -6.40720400 & -0.31801200 \\
\hline C & 5.12842000 & -3.98205200 & 0.62254700 \\
\hline C & 6.09536800 & -1.49685700 & 1.59627200 \\
\hline $\mathrm{H}$ & 5.67901400 & 3.48889900 & 1.67115500 \\
\hline $\mathrm{H}$ & 6.68896900 & -2.27230100 & 1.11854600 \\
\hline $\mathrm{H}$ & 5.57606900 & -3.97355900 & 1.61843100 \\
\hline $\mathrm{H}$ & 1.72572600 & -6.74821100 & -1.34792700 \\
\hline $\mathrm{H}$ & -0.53171700 & -7.09681700 & -0.91697800 \\
\hline $\mathrm{H}$ & -5.21908100 & -5.04562400 & 0.05903300 \\
\hline $\mathrm{H}$ & -5.68274600 & -3.48719400 & 1.66834300 \\
\hline $\mathrm{H}$ & -5.74459900 & 1.91620300 & 2.54306300 \\
\hline $\mathrm{H}$ & -5.57511900 & 3.97347700 & 1.61844200 \\
\hline $\mathrm{H}$ & -1.12249200 & 6.99949500 & 0.27085000 \\
\hline $\mathrm{H}$ & 0.54775800 & 7.09506800 & -0.92372900 \\
\hline $\mathrm{H}$ & 5.21781700 & 5.04728600 & 0.05950200 \\
\hline C & -7.12290000 & -3.03688900 & 0.09627100 \\
\hline $\mathrm{H}$ & -7.27413600 & -4.11379600 & 0.00681500 \\
\hline $\mathrm{H}$ & -8.02602600 & -2.62104200 & 0.55247300 \\
\hline $\mathrm{H}$ & -7.05327900 & -2.63851000 & -0.91865000 \\
\hline C & -4.21557800 & -5.80491500 & -1.66883100 \\
\hline C & -0.26556600 & -6.16040300 & -2.85718600 \\
\hline C & 3.22338800 & -6.81367900 & 0.15532300 \\
\hline C & 6.19324100 & -4.12231500 & -0.46838000 \\
\hline C & 7.10026200 & -0.38649400 & 1.91554600 \\
\hline C & 7.12262600 & 3.03787200 & 0.10248800 \\
\hline C & 4.21658400 & 5.80177000 & -1.67246800 \\
\hline $\mathrm{C}$ & 0.27327500 & 6.15923700 & -2.86223000 \\
\hline C & -3.21860000 & 6.81538400 & 0.16012800 \\
\hline C & -6.19151300 & 4.12537000 & -0.46840500 \\
\hline C & -7.10332000 & 0.38820400 & 1.90947900 \\
\hline $\mathrm{H}$ & -6.77470600 & 3.21419600 & -0.62067000 \\
\hline $\mathrm{H}$ & -6.88288800 & 4.93050800 & -0.20716500 \\
\hline $\mathrm{H}$ & -5.74572400 & 4.36958500 & -1.43586400 \\
\hline $\mathrm{H}$ & -3.39573000 & 6.57056300 & 1.20860900 \\
\hline $\mathrm{H}$ & -3.29032300 & 7.90374600 & 0.07723200 \\
\hline $\mathrm{H}$ & -4.02698000 & 6.40766700 & -0.44967600 \\
\hline
\end{tabular}




$\begin{array}{lrrr}\mathrm{H} & 0.53380800 & 5.24856300 & -3.40487400 \\ \mathrm{H} & -0.80973800 & 6.26357000 & -2.94300600 \\ \mathrm{H} & 0.72728700 & 7.00065600 & -3.39246100 \\ \mathrm{H} & 3.62510500 & 5.47555800 & -2.53018800 \\ \mathrm{H} & 3.66916800 & 6.57569600 & -1.13163000 \\ \mathrm{H} & 5.11409600 & 6.28139600 & -2.07343100 \\ \mathrm{H} & 8.02499000 & 2.62295500 & 0.56103300 \\ \mathrm{H} & 7.05540500 & 2.63843400 & -0.91217800 \\ \mathrm{H} & 7.27350200 & 4.11476600 & 0.01230600 \\ \mathrm{H} & 7.97257500 & -0.85761000 & 2.37814100 \\ \mathrm{H} & 7.46216800 & 0.13196200 & 1.02590600 \\ \mathrm{H} & 6.73564600 & 0.34944900 & 2.63184300 \\ \mathrm{H} & 6.88386500 & -4.92863100 & -0.20879600 \\ \mathrm{H} & 5.74777700 & -4.36369600 & -1.43670400 \\ \mathrm{H} & 6.77715800 & -3.21116400 & -0.61796600 \\ \mathrm{H} & 3.29562900 & -7.90203700 & 0.07271200 \\ \mathrm{H} & 4.02826800 & -6.40567700 & -0.45888200 \\ \mathrm{H} & 3.40574700 & -6.56825500 & 1.20274500 \\ \mathrm{H} & -0.62584100 & -7.06131100 & -3.36059000 \\ \mathrm{H} & -0.62158700 & -5.30063800 & -3.42787500 \\ \mathrm{H} & 0.82286100 & -6.14656000 & -2.93418000 \\ \mathrm{H} & -3.66875100 & -6.57711800 & -1.12501200 \\ \mathrm{H} & -5.11295400 & -6.28568600 & -2.06873800 \\ \mathrm{H} & -3.62341300 & -5.48208900 & -2.52730600 \\ \mathrm{H} & -6.74040000 & -0.34871400 & 2.62563100 \\ \mathrm{H} & -7.97589200 & 0.85967600 & 2.37122400 \\ \mathrm{H} & -7.46438100 & -0.12908300 & 1.01881300 \\ \mathrm{C} & -0.00371600 & 0.00410900 & -0.24956700 \\ \mathrm{O} & 1.06587200 & 0.46447400 & -0.25724400 \\ \mathrm{O} & -1.07272400 & -0.45712800 & -0.22331300\end{array}$

$\begin{array}{lrrr}\mathrm{CO}_{2} @(\mathrm{OH})_{12} \mathrm{CB}[6] & & \\ \mathrm{C} & -3.10689000 & -4.00692800 & -0.02568400 \\ \mathrm{C} & -1.68919500 & -4.69640800 & -0.02868300 \\ \mathrm{~N} & -1.12592000 & -4.28137700 & 1.22419200 \\ \mathrm{C} & -1.96722500 & -3.44476200 & 1.94225100 \\ \mathrm{~N} & -3.08540000 & -3.19726200 & 1.15334800 \\ \mathrm{~N} & -3.14307800 & -3.35184600 & -1.29606300 \\ \mathrm{~N} & -1.06347600 & -4.18010100 & -1.20551200 \\ \mathrm{C} & 0.09472000 & -4.80377800 & 1.78367300 \\ \mathrm{O} & -1.78323500 & -3.04148800 & 3.06411100 \\ \mathrm{C} & 0.16375000 & -4.70652800 & -1.75213100 \\ \mathrm{C} & -1.94014800 & -3.44347600 & -1.98755400 \\ \mathrm{O} & -1.71839900 & -3.00164300 & -3.08758300 \\ \mathrm{~N} & 1.35086700 & -4.13257700 & -1.16624300 \\ \mathrm{C} & 1.94725900 & -4.60674000 & 0.04346900 \\ \mathrm{C} & 2.23479500 & -3.38532600 & -1.93163800 \\ \mathrm{C} & 3.32294400 & -3.83819000 & 0.10409100 \\ \mathrm{~N} & 1.30839300 & -4.22244300 & 1.26855100 \\ \mathrm{~N} & 3.38411700 & -3.19459900 & -1.17485600 \\ \mathrm{O} & 2.05721200 & -3.00819000 & -3.06337600 \\ \mathrm{~N} & 3.19312200 & -3.01605900 & 1.26287400 \\ \mathrm{C} & 2.04939400 & -3.29785400 & 1.99333400 \\ \mathrm{O} & 1.76610700 & -2.85605200 & 3.07978600 \\ \mathrm{C} & 4.48910100 & -2.40766600 & -1.66064100 \\ \mathrm{C} & 4.25607500 & -2.21440600 & 1.81391200 \\ & & & \end{array}$




\begin{tabular}{|c|c|c|c|}
\hline $\mathrm{N}$ & 4.36253100 & -0.90045500 & 1.23048900 \\
\hline $\mathrm{N}$ & 4.54249900 & -1.04324200 & -1.20379200 \\
\hline $\mathrm{C}$ & 4.07025900 & 0.24077200 & 1.96283400 \\
\hline $\mathrm{C}$ & 5.10055600 & -0.61337500 & 0.04348600 \\
\hline C & 5.02797700 & 0.95594200 & -0.06112200 \\
\hline $\mathrm{N}$ & 4.45539300 & 1.33517600 & 1.19674400 \\
\hline $\mathrm{C}$ & 4.03954800 & 0.00973600 & -1.95896700 \\
\hline $\mathrm{N}$ & 4.23708400 & 1.17439900 & -1.23026900 \\
\hline $\mathrm{C}$ & 4.32286100 & 2.68812000 & 1.67207000 \\
\hline $\mathrm{C}$ & 4.03126700 & 2.47104900 & -1.82625500 \\
\hline $\mathrm{O}$ & 3.56485700 & -0.07165800 & -3.06435800 \\
\hline $\mathrm{O}$ & 3.61201000 & 0.28002600 & 3.07761700 \\
\hline $\mathrm{N}$ & 3.17786600 & 3.41675700 & 1.18735700 \\
\hline $\mathrm{N}$ & 2.94200500 & 3.21422200 & -1.23870100 \\
\hline $\mathrm{C}$ & 2.02668800 & 3.57036000 & 1.95068000 \\
\hline $\mathrm{C}$ & 3.08408500 & 4.05577000 & -0.09211800 \\
\hline $\mathrm{C}$ & 1.69877200 & 4.80230300 & -0.01119900 \\
\hline $\mathrm{N}$ & 1.11833700 & 4.29378200 & 1.19057500 \\
\hline $\mathrm{O}$ & 1.86562600 & 3.18227900 & 3.08110600 \\
\hline $\mathrm{C}$ & -0.09876600 & 4.80401700 & 1.77213200 \\
\hline $\mathrm{N}$ & 1.06855600 & 4.44277500 & -1.24738900 \\
\hline $\mathrm{C}$ & 1.80816400 & 3.52149400 & -1.97877300 \\
\hline $\mathrm{O}$ & 1.53191700 & 3.09902700 & -3.07429600 \\
\hline $\mathrm{C}$ & -0.17481300 & 4.98476200 & -1.73443800 \\
\hline $\mathrm{N}$ & -1.29465300 & 4.21000100 & 1.22625100 \\
\hline $\mathrm{N}$ & -1.37095800 & 4.37891000 & -1.20599200 \\
\hline C & -2.11103900 & 3.39878600 & 2.00010400 \\
\hline $\mathrm{C}$ & -1.96128100 & 4.69832000 & 0.06125100 \\
\hline $\mathrm{C}$ & -3.30937000 & 3.88179700 & 0.03708000 \\
\hline $\mathrm{N}$ & -3.29902300 & 3.21404200 & 1.30145400 \\
\hline $\mathrm{O}$ & -1.85910300 & 2.96754400 & 3.09776100 \\
\hline $\mathrm{C}$ & -4.33099900 & 2.32983800 & 1.77740300 \\
\hline $\mathrm{N}$ & -3.19531500 & 3.07587200 & -1.14246700 \\
\hline $\mathrm{C}$ & -2.11809700 & 3.46478700 & -1.93449300 \\
\hline $\mathrm{O}$ & -1.89801900 & 3.10548500 & -3.06454000 \\
\hline $\mathrm{C}$ & -4.31031400 & 2.36948000 & -1.73425000 \\
\hline $\mathrm{N}$ & -4.33022400 & 1.01355800 & 1.18068300 \\
\hline $\mathrm{N}$ & -4.49531900 & 1.02500200 & -1.25620800 \\
\hline C & -5.07774300 & 0.68227000 & 0.00427600 \\
\hline $\mathrm{C}$ & -4.02842200 & -0.07901500 & -1.95604800 \\
\hline $\mathrm{C}$ & -5.05940900 & -0.89264300 & -0.01914900 \\
\hline $\mathrm{N}$ & -4.28524900 & -1.20540900 & -1.18146800 \\
\hline $\mathrm{C}$ & -4.07049000 & -0.10786900 & 1.96305600 \\
\hline $\mathrm{N}$ & -4.49983600 & -1.22198600 & 1.25468000 \\
\hline $\mathrm{O}$ & -3.53934700 & -0.06696500 & -3.05804700 \\
\hline $\mathrm{O}$ & -3.60677500 & -0.10870500 & 3.07584700 \\
\hline $\mathrm{C}$ & -4.22264100 & -2.52370000 & -1.76738100 \\
\hline $\mathrm{C}$ & -4.25298700 & -2.56290800 & 1.71780100 \\
\hline $\mathrm{H}$ & 4.95096600 & 3.05587600 & -1.74961700 \\
\hline $\mathrm{H}$ & 3.77452000 & 2.30214300 & -2.87396300 \\
\hline $\mathrm{H}$ & 4.22196100 & 2.63322200 & 2.75813800 \\
\hline $\mathrm{H}$ & 5.22573200 & 3.23955800 & 1.39530500 \\
\hline $\mathrm{H}$ & -0.14126900 & 5.88746600 & 1.63735200 \\
\hline $\mathrm{H}$ & -0.07122400 & 4.55275800 & 2.83441500 \\
\hline $\mathrm{H}$ & -0.19878500 & 6.05318400 & -1.50300700 \\
\hline $\mathrm{H}$ & -0.19093300 & 4.82318100 & -2.81443600 \\
\hline $\mathrm{H}$ & -5.30450200 & 2.79509600 & 1.60481400 \\
\hline $\mathrm{H}$ & -4.15733500 & 2.19351200 & 2.84676000 \\
\hline $\mathrm{H}$ & -5.22566400 & 2.93730600 & -1.55152200 \\
\hline $\mathrm{H}$ & -4.11029600 & 2.29906500 & -2.80526100 \\
\hline $\mathrm{H}$ & -4.09193600 & -2.49754800 & 2.79568900 \\
\hline
\end{tabular}




$\begin{array}{lrrr}\mathrm{H} & -5.12793300 & -3.17904100 & 1.49721000 \\ \mathrm{H} & -4.06585300 & -2.38922700 & -2.83948800 \\ \mathrm{H} & -5.17056300 & -3.03465300 & -1.58202400 \\ \mathrm{H} & 0.12475300 & -5.88193700 & 1.60328200 \\ \mathrm{H} & 0.06849900 & -4.59112000 & 2.85461500 \\ \mathrm{H} & 0.17871100 & -4.44792500 & -2.81287400 \\ \mathrm{H} & 0.18148500 & -5.79122900 & -1.62217900 \\ \mathrm{H} & 5.42000700 & -2.90246600 & -1.37030500 \\ \mathrm{H} & 4.39409000 & -2.37469600 & -2.74805300 \\ \mathrm{H} & 4.03499100 & -2.07313900 & 2.87371000 \\ \mathrm{H} & 5.20461300 & -2.74226900 & 1.69065000 \\ \mathrm{O} & 4.40999000 & -4.69896500 & 0.30619700 \\ \mathrm{O} & 2.11167200 & -5.99010100 & -0.06004800 \\ \mathrm{O} & -1.76865400 & -6.08492800 & -0.14227100 \\ \mathrm{O} & -4.15011400 & -4.93511800 & 0.11184400 \\ \mathrm{O} & -6.33550800 & -1.44253400 & -0.18879300 \\ \mathrm{O} & -6.36777400 & 1.20256300 & 0.14846100 \\ \mathrm{O} & -4.42967300 & 4.70933700 & -0.11206800 \\ \mathrm{O} & -2.16632400 & 6.07168400 & 0.21560400 \\ \mathrm{O} & 1.84333900 & 6.18712200 & 0.12301100 \\ \mathrm{O} & 4.15108500 & 4.93161900 & -0.32099800 \\ \mathrm{O} & 6.28401100 & 1.53827000 & -0.26408500 \\ \mathrm{O} & 6.40902400 & -1.08066700 & 0.21915200 \\ \mathrm{H} & 4.50459600 & -5.22715500 & -0.49528400 \\ \mathrm{H} & 2.59360300 & -6.27614400 & 0.72532800 \\ \mathrm{H} & -2.34795600 & -6.39166000 & 0.56596000 \\ \mathrm{H} & -4.23840400 & -5.38634900 & -0.73571600 \\ \mathrm{H} & -6.83229800 & -1.25126600 & 0.61522800 \\ \mathrm{H} & -6.85714200 & 0.96417700 & -0.64755600 \\ \mathrm{H} & -4.54287300 & 5.18320900 & 0.72005700 \\ \mathrm{H} & -2.70470600 & 6.36118500 & -0.53085100 \\ \mathrm{H} & 2.26212700 & 6.50581200 & -0.68557500 \\ \mathrm{H} & 4.22455100 & 5.49498500 & 0.45856600 \\ \mathrm{H} & 6.81215900 & 1.34700700 & 0.52025400 \\ \mathrm{H} & 6.89196200 & -0.87218200 & -0.58954800 \\ \mathrm{C} & 0.05192700 & -1.00738200 & -0.13104300 \\ \mathrm{O} & -1.11209800 & -1.04874700 & -0.10910200 \\ \mathrm{O} & 1.21595100 & -0.96326900 & -0.14664900\end{array}$

\section{$\mathrm{CO}_{2} @ \mathrm{CB}[6] \mathrm{CN}$}

$\begin{array}{llll}\text { C } & 5.11804300 & -0.34495600 & 0.15803300\end{array}$

$\begin{array}{llll}\mathrm{C} & 4.76312400 & -1.90299600 & 0.15003600\end{array}$

$\mathrm{N} \quad 4.13550700 \quad-2.07972100 \quad-1.13135600$

C $\quad 4.13154200 \quad-0.92826000 \quad-1.90843800$

$\mathrm{N} \quad 4.66819500 \quad 0.09368600 \quad-1.13415400$

N $\quad \begin{array}{llll}\mathrm{N} & 4.38171600 & 0.14436300 & 1.29154300\end{array}$

$\mathrm{N} \quad 3.92322800 \quad-2.04543200 \quad 1.30551500$

C $\quad 3.74102200 \quad-3.35162400 \quad-1.68651600$

$\begin{array}{llll}\text { O } & 3.77232700 & -0.84090800 & -3.05185900\end{array}$

C $\quad 3.45349200 \quad-3.29919000 \quad 1.83628400$

C $\quad 3.69721800 \quad-0.85195600 \quad 1.97555800$

$\begin{array}{llll}\mathrm{O} & 3.06428700 & -0.71239400 & 2.98850900\end{array}$

N $\quad 2.25407000 \quad-3.80212100 \quad 1.21104400$

C $\quad 2.23052100-4.56082500-0.01290700$

C $\quad \begin{array}{llll}\text { C } & 1.06725600 & -3.88383000 & 1.92754000\end{array}$

$\begin{array}{llll}\text { C } & 0.69320700 & -4.96875100 & -0.12976800\end{array}$ 


\begin{tabular}{|c|c|c|c|}
\hline $\mathrm{N}$ & 2.46261400 & -3.83980100 & -1.23112400 \\
\hline $\mathrm{N}$ & 0.16005600 & -4.58953000 & 1.14642200 \\
\hline $\mathrm{O}$ & 0.87065400 & -3.45874200 & 3.03481500 \\
\hline $\mathrm{N}$ & 0.25019000 & -4.18889000 & -1.25940500 \\
\hline $\mathrm{C}$ & 1.30251000 & -3.59030300 & -1.94715200 \\
\hline $\mathrm{O}$ & 1.22150800 & -2.99869900 & -2.99090200 \\
\hline $\mathrm{C}$ & -1.16251600 & -4.90735100 & 1.62895300 \\
\hline $\mathrm{C}$ & -1.02968000 & -4.35932300 & -1.90524700 \\
\hline $\mathrm{N}$ & -2.10805600 & -3.64681800 & -1.26240500 \\
\hline $\mathrm{N}$ & -2.21348700 & -4.04585500 & 1.14383600 \\
\hline $\mathrm{C}$ & -2.78458800 & -2.64254600 & -1.94960400 \\
\hline $\mathrm{C}$ & -2.85321500 & -4.15241000 & -0.13588000 \\
\hline $\mathrm{C}$ & -4.05566000 & -3.10988800 & -0.02339900 \\
\hline $\mathrm{N}$ & -3.94126300 & -2.35973800 & -1.24058500 \\
\hline C & -2.72703700 & -3.01877400 & 1.92647600 \\
\hline $\mathrm{N}$ & -3.75049700 & -2.41964800 & 1.20373200 \\
\hline $\mathrm{C}$ & -4.86162900 & -1.34381600 & -1.68820800 \\
\hline $\mathrm{C}$ & -4.60875400 & -1.43931300 & 1.82502100 \\
\hline $\mathrm{O}$ & -2.37422200 & -2.73007300 & 3.03889800 \\
\hline $\mathrm{O}$ & -2.44361800 & -2.14151600 & -2.98813400 \\
\hline $\mathrm{N}$ & -4.63282900 & -0.03325200 & -1.12962000 \\
\hline $\mathrm{N}$ & -4.45946200 & -0.10292400 & 1.30938500 \\
\hline C & -4.11271100 & 0.99357300 & -1.90780900 \\
\hline $\mathrm{C}$ & -5.12491900 & 0.41110000 & 0.14657400 \\
\hline $\mathrm{C}$ & -4.72394500 & 1.95744100 & 0.16116600 \\
\hline $\mathrm{N}$ & -4.13500000 & 2.14842600 & -1.13511200 \\
\hline $\mathrm{O}$ & -3.75199900 & 0.90934000 & -3.05098400 \\
\hline $\mathrm{C}$ & -3.73241300 & 3.42099500 & -1.68232600 \\
\hline $\mathrm{N}$ & -3.83404300 & 2.04526100 & 1.28776600 \\
\hline $\mathrm{C}$ & -3.69589900 & 0.84503800 & 1.97399700 \\
\hline $\mathrm{O}$ & -3.06803700 & 0.67252800 & 2.98501200 \\
\hline $\mathrm{C}$ & -3.34739500 & 3.27839700 & 1.85166500 \\
\hline $\mathrm{N}$ & -2.44849100 & 3.90038900 & -1.22917100 \\
\hline $\mathrm{N}$ & -2.16835900 & 3.80056000 & 1.19986500 \\
\hline $\mathrm{C}$ & -1.28822500 & 3.64734200 & -1.94704800 \\
\hline $\mathrm{C}$ & -2.20182100 & 4.59905400 & -0.00189500 \\
\hline $\mathrm{C}$ & -0.68109900 & 5.04660400 & -0.14644700 \\
\hline $\mathrm{N}$ & -0.23403700 & 4.24218700 & -1.25841200 \\
\hline $\mathrm{O}$ & -1.21180400 & 3.05531800 & -2.99071600 \\
\hline $\mathrm{C}$ & 1.04363800 & 4.40993200 & -1.90925000 \\
\hline $\mathrm{N}$ & -0.12969600 & 4.72780400 & 1.13823400 \\
\hline $\mathrm{C}$ & -0.99206900 & 3.97227200 & 1.92361200 \\
\hline $\mathrm{O}$ & -0.77534300 & 3.57155500 & 3.03584000 \\
\hline $\mathrm{C}$ & 1.20018700 & 5.06353800 & 1.58923400 \\
\hline $\mathrm{N}$ & 2.12448000 & 3.70401100 & -1.26431900 \\
\hline $\mathrm{N}$ & 2.24683100 & 4.17990500 & 1.13322500 \\
\hline $\mathrm{C}$ & 2.88066400 & 4.22813300 & -0.15288400 \\
\hline $\mathrm{C}$ & 2.70089700 & 3.12908600 & 1.92111800 \\
\hline $\mathrm{C}$ & 4.05220300 & 3.15766000 & -0.01208100 \\
\hline $\mathrm{N}$ & 3.68012500 & 2.45917900 & 1.19422400 \\
\hline $\mathrm{C}$ & 2.80319100 & 2.70076700 & -1.95059800 \\
\hline $\mathrm{N}$ & 3.95956900 & 2.41862300 & -1.23733200 \\
\hline $\mathrm{O}$ & 2.34000400 & 2.86867100 & 3.03756100 \\
\hline $\mathrm{O}$ & 2.46693200 & 2.19737500 & -2.98938200 \\
\hline $\mathrm{C}$ & 4.50894000 & 1.46904900 & 1.84285700 \\
\hline $\mathrm{C}$ & 4.88947900 & 1.40909100 & -1.68389000 \\
\hline $\mathrm{H}$ & -5.65669600 & -1.74701000 & 1.73255300 \\
\hline $\mathrm{H}$ & -4.32677300 & -1.40780900 & 2.87992000 \\
\hline $\mathrm{H}$ & -4.72585300 & -1.24457700 & -2.76714200 \\
\hline $\mathrm{H}$ & -5.88537000 & -1.66860600 & -1.47310800 \\
\hline $\mathrm{H}$ & -4.49269000 & 4.17812300 & -1.46399300 \\
\hline
\end{tabular}




\begin{tabular}{lrrr}
$\mathrm{H}$ & -3.65281900 & 3.27765400 & -2.76187900 \\
$\mathrm{H}$ & -4.14700500 & 4.02890900 & 1.83997400 \\
$\mathrm{H}$ & -3.05992600 & 3.06658600 & 2.88436000 \\
$\mathrm{H}$ & 1.28611500 & 5.47706000 & -1.99029000 \\
$\mathrm{H}$ & 0.94238800 & 3.98061400 & -2.90939300 \\
$\mathrm{H}$ & 1.43509800 & 6.09012400 & 1.29131700 \\
$\mathrm{H}$ & 1.18511500 & 4.98959600 & 2.67840000 \\
$\mathrm{H}$ & 4.76046100 & 1.31394200 & -2.76396600 \\
$\mathrm{H}$ & 5.90923400 & 1.73982700 & -1.46057900 \\
$\mathrm{H}$ & 4.16985700 & 1.41765000 & 2.88042100 \\
$\mathrm{H}$ & 5.56052600 & 1.77911900 & 1.81396600 \\
$\mathrm{H}$ & 4.50723100 & -4.10559800 & -1.47651800 \\
$\mathrm{H}$ & 3.65670200 & -3.20204800 & -2.76492900 \\
$\mathrm{H}$ & 3.20441500 & -3.12684300 & 2.88580300 \\
$\mathrm{H}$ & 4.25259700 & -4.04629500 & 1.76559200 \\
$\mathrm{H}$ & -1.39939700 & -5.94752200 & 1.38236300 \\
$\mathrm{H}$ & -1.13499700 & -4.78200600 & 2.71336200 \\
$\mathrm{H}$ & -0.93030700 & -3.93616100 & -2.90819000 \\
$\mathrm{H}$ & -1.27390400 & -5.42637500 & -1.97781400 \\
$\mathrm{C}$ & -6.58315300 & 0.16520600 & 0.24253400 \\
$\mathrm{C}$ & -5.87341500 & 2.87501100 & 0.33915800 \\
$\mathrm{C}$ & -3.15013000 & 5.72582600 & 0.15163500 \\
$\mathrm{C}$ & -0.50232500 & 6.48653200 & -0.44082100 \\
$\mathrm{C}$ & 3.35050100 & 5.60142600 & -0.44462100 \\
$\mathrm{C}$ & 5.39976300 & 3.75438000 & 0.13170000 \\
$\mathrm{C}$ & 6.56260700 & -0.05874100 & 0.32019400 \\
$\mathrm{C}$ & 5.94400000 & -2.79131600 & 0.26232000 \\
$\mathrm{C}$ & 3.15724700 & -5.71259000 & 0.08279700 \\
$\mathrm{C}$ & 0.47436900 & -6.41215600 & -0.37531400 \\
$\mathrm{C}$ & -3.28971900 & -5.54519500 & -0.38298300 \\
$\mathrm{C}$ & -5.39477300 & -3.73704300 & 0.06664500 \\
$\mathrm{~N}$ & -6.72077500 & 3.65059600 & 0.47435800 \\
$\mathrm{~N}$ & -3.94243300 & 6.55577600 & 0.29683600 \\
$\mathrm{~N}$ & -0.28963400 & 7.59610800 & -0.68832000 \\
$\mathrm{~N}$ & 3.64419200 & 6.69302600 & -0.68915100 \\
$\mathrm{~N}$ & 6.47741100 & 4.15099400 & 0.26965500 \\
$\mathrm{~N}$ & 7.67274100 & 0.24234400 & 0.44177200 \\
$\mathrm{~N}$ & 6.81526800 & -3.54805200 & 0.34037400 \\
$\mathrm{~N}$ & 3.93893900 & -6.55954400 & 0.17954800 \\
$\mathrm{~N}$ & 0.23315300 & -7.52463700 & -0.57990900 \\
$\mathrm{~N}$ & -3.56025700 & -6.65078400 & -0.58818900 \\
& -6.46962200 & -4.15394000 & 0.15961500 \\
$\mathrm{~N}$ & -7.70570800 & -0.10617100 & 0.30647500 \\
-0.24485900 & -0.90082100 & -0.01816300 \\
\hline & -1.37937400 & -1.16786400 & -0.00508400 \\
\hline & & -0.63632400 & -0.02924800
\end{tabular}

